\title{
Search for top squark pair production in final states with one isolated lepton, jets, and missing transverse momentum in $\sqrt{s}=8 \mathrm{TeV} p p$ collisions with the ATLAS detector
}

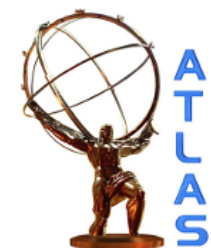

\section{The ATLAS collaboration}

E-mail: atlas.publications@cern.ch

ABSTRACT: The results of a search for top squark (stop) pair production in final states with one isolated lepton, jets, and missing transverse momentum are reported. The analysis is performed with proton-proton collision data at $\sqrt{s}=8 \mathrm{TeV}$ collected with the ATLAS detector at the LHC in 2012 corresponding to an integrated luminosity of $20 \mathrm{fb}^{-1}$. The lightest supersymmetric particle (LSP) is taken to be the lightest neutralino which only interacts weakly and is assumed to be stable. The stop decay modes considered are those to a top quark and the LSP as well as to a bottom quark and the lightest chargino, where the chargino decays to the LSP by emitting a $W$ boson. A wide range of scenarios with different mass splittings between the stop, the lightest neutralino and the lightest chargino are considered, including cases where the $W$ bosons or the top quarks are off-shell. Decay modes involving the heavier charginos and neutralinos are addressed using a set of phenomenological models of supersymmetry. No significant excess over the Standard Model prediction is observed. A stop with a mass between 210 and $640 \mathrm{GeV}$ decaying directly to a top quark and a massless LSP is excluded at $95 \%$ confidence level, and in models where the mass of the lightest chargino is twice that of the LSP, stops are excluded at $95 \%$ confidence level up to a mass of $500 \mathrm{GeV}$ for an LSP mass in the range of 100 to $150 \mathrm{GeV}$. Stringent exclusion limits are also derived for all other stop decay modes considered, and model-independent upper limits are set on the visible cross-section for processes beyond the Standard Model.

KEYWORDS: Hadron-Hadron Scattering, proton-proton scattering, supersymmetry, top squark

ARXIV EPRINT: 1407.0583 


\section{Contents}

$\begin{array}{llr}1 & \text { Introduction } & 2\end{array}$

2 Analysis strategy 3

3 The ATLAS detector 5

4 Trigger and data collection $\quad 6$

$\begin{array}{llr}5 & \text { Simulated samples } & \mathbf{7}\end{array}$

$\begin{array}{ll}5.1 \text { Background samples } & 7\end{array}$

5.2 Signal samples 8

6 Physics object reconstruction and discriminating variables 10

$\begin{array}{lll}6.1 \text { Physics object reconstruction } & 10\end{array}$

6.2 Tools to discriminate signal from background 14

$\begin{array}{llr}7 & \text { Signal selections } & \mathbf{1 6}\end{array}$

$\begin{array}{lll}7.1 & \text { Event preselection } & 17\end{array}$

$\begin{array}{lll}7.2 & \text { Selections for the } \tilde{t}_{1} \rightarrow t \tilde{\chi}_{1}^{0} \text { decay } & 18\end{array}$

$\begin{array}{ll}\text { 7.3 Selections for the } \tilde{t}_{1} \rightarrow b \tilde{\chi}_{1}^{ \pm} \text {decay } & 21\end{array}$

$\begin{array}{lll}7.4 & \text { Selections for the mixed, three- and four-body decays }\end{array}$

8 Background estimates $\quad 28$

$\begin{array}{ll}\text { 8.1 Control regions } & 29\end{array}$

8.2 Validation 32

9 Systematic uncertainties $\quad 35$

10 Results $\quad 38$

$\begin{array}{ll}11 \text { Summary and conclusions } & 45\end{array}$

A Detailed description of the discriminating variables 53

$\begin{array}{ll}\text { B Background fit results } & 56\end{array}$

$\begin{array}{ll}\text { The ATLAS collaboration } & 70\end{array}$ 


\section{Introduction}

The hierarchy problem [1-4] has gained additional attention with the observation of a new particle consistent with the Standard Model (SM) Higgs boson [5, 6] at the LHC [7]. Supersymmetry (SUSY) [8-16], which extends the SM by introducing supersymmetric partners for all SM particles, provides an elegant solution to the hierarchy problem. The partner particles have identical quantum numbers except for a half-unit difference in spin. The superpartners of the left- and right-handed top quarks, $\tilde{t}_{\mathrm{L}}$ and $\tilde{t}_{\mathrm{R}}$, mix to form the two mass eigenstates $\tilde{t}_{1}$ and $\tilde{t}_{2}$, where $\tilde{t}_{1}$ (top squark or stop) is the lighter one. If the supersymmetric partners of the top quarks have masses $\lesssim 1 \mathrm{TeV}$, loop diagrams involving top quarks, which are the dominant contribution to the divergence of the Higgs boson mass, can be largely cancelled [17-24]. Significant mass splitting between $\tilde{t}_{1}$ and $\tilde{t}_{2}$ is possible due to the large top Yukawa coupling. ${ }^{1}$ Furthermore, effects of the renormalisation group equations are strong for the third generation squarks, usually driving their masses significantly lower than those of the other generations. These considerations suggest a light stop which, together with the stringent LHC limits excluding other coloured supersymmetric particles up to masses at the $\mathrm{TeV}$ level, motivates dedicated stop searches.

SUSY models can violate the conservation of baryon number and lepton number, resulting in a proton lifetime shorter than current experimental limits [25]. This is commonly solved by introducing a multiplicative quantum number called $R$-parity, which is 1 and -1 for all SM and SUSY particles, respectively. A generic $R$-parity-conserving minimal supersymmetric extension of the SM (MSSM) [17, 26-29] predicts pair production of SUSY particles and the existence of a stable lightest supersymmetric particle (LSP). In a large variety of SUSY models, the lightest neutralino ${ }^{2}\left(\tilde{\chi}_{1}^{0}\right)$ is the LSP, which is also the assumption throughout this paper. Since the $\tilde{\chi}_{1}^{0}$ interacts only weakly it is a candidate for dark matter.

The stop can decay into a variety of final states, depending amongst other things on the SUSY particle mass spectrum, in particular on the masses of the stop and the lightest neutralino. Figure 1 illustrates the simplest decay modes as a function of the stop and LSP masses. In the rightmost wedge, the stop mass is greater than the sum of the top quark and the LSP masses, hence the decay $\tilde{t}_{1} \rightarrow t \tilde{\chi}_{1}^{0}$ is kinematically allowed. A lighter stop can undergo a three-body decay $\tilde{t}_{1} \rightarrow b W \tilde{\chi}_{1}^{0}$ if the stop mass is still above the $b+W+\tilde{\chi}_{1}^{0}$ mass. For an even lighter stop, the decay proceeds via a four-body process $\tilde{t}_{1} \rightarrow b f f^{\prime} \tilde{\chi}_{1}^{0}$, where $f$ and $f^{\prime}$ are two distinct fermions, or flavour-changing neutral current (FCNC) processes, such as the loop-suppressed $\tilde{t}_{1} \rightarrow c \tilde{\chi}_{1}^{0}$. If supersymmetric particles other than the $\tilde{\chi}_{1}^{0}$ are lighter than the stop, then additional decay modes can open up. The stop decay to a bottom quark and the lightest chargino $\left(\tilde{t}_{1} \rightarrow b \tilde{\chi}_{1}^{ \pm}\right)$is an important example, where the $\tilde{\chi}_{1}^{ \pm}$can decay to the lightest neutralino by emitting an on- or off-shell $W$ boson

\footnotetext{
${ }^{1}$ The masses of the $\tilde{t}_{1}$ and $\tilde{t}_{2}$ are given by the eigenvalues of the stop mass matrix. The stop mass matrix involves the top-quark Yukawa coupling in the off-diagonal elements, which typically induces a large mass splitting. The stop mass matrix is diagonalised by the stop mixing matrix, which gives the $\tilde{t}_{\mathrm{L}}$ and $\tilde{t}_{\mathrm{R}}$ components of the mass eigenstates $\tilde{t}_{1}$ and $\tilde{t}_{2}$.

${ }^{2}$ The charginos $\tilde{\chi}_{1,2}^{ \pm}$and neutralinos $\tilde{\chi}_{1,2,3,4}^{0}$ are the mass eigenstates formed from the linear superposition of the charged and neutral SUSY partners of the Higgs and electroweak gauge bosons (higgsinos, winos and binos).
} 


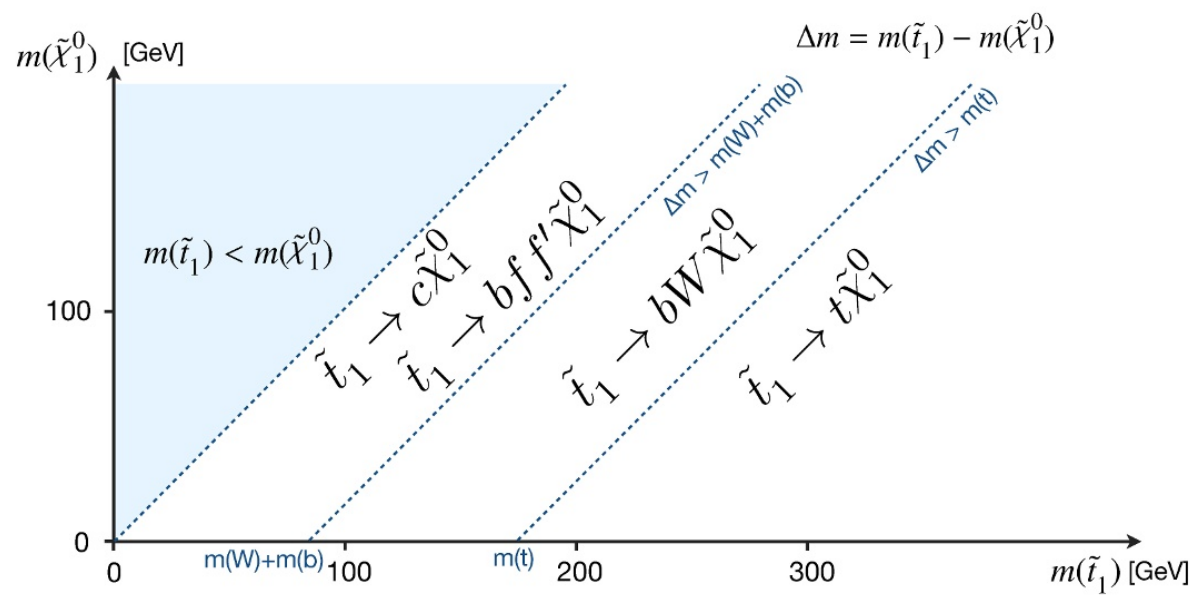

Figure 1. Illustration of stop decay modes in the plane spanned by the masses of the stop $\left(\tilde{t}_{1}\right)$ and the lightest neutralino $\left(\tilde{\chi}_{1}^{0}\right)$, where the latter is assumed to be the lightest supersymmetric particle. Stop decays to supersymmetric particles other than the lightest supersymmetric particle are not displayed.

$\left(\tilde{\chi}_{1}^{ \pm} \rightarrow W^{(*)} \tilde{\chi}_{1}^{0}\right)$. The $\tilde{t}_{1} \rightarrow b \tilde{\chi}_{1}^{ \pm}$decay is considered for a stop mass above around $100 \mathrm{GeV}$ since the LEP limit on the lightest chargino is $m_{\tilde{\chi}_{1}^{ \pm}}>103.5 \mathrm{GeV}[30]$.

This article presents a search for direct $\tilde{t}_{1}$ pair production in final states with exactly one isolated charged lepton (electron or muon, ${ }^{3}$ henceforth referred to simply as 'leptons'), several jets, and a significant amount of missing transverse momentum, the magnitude of which is referred to as $E_{\mathrm{T}}^{\mathrm{miss}}$. The lepton arises from the decay of either a real or a virtual $W$ boson, and the potentially large $E_{\mathrm{T}}^{\text {miss }}$ is generated by the two undetected LSPs and neutrino(s). All stop decay modes described above except for the FCNC modes are considered, as illustrated in figure 2 . With several decay modes kinematically available, the $\tilde{t}_{1}$ decay branching ratio is determined by factors including the stop mixing matrix and the field content of the neutralino/chargino sector. Results are mainly based on simplified models that have $100 \%$ branching ratio to one or a pair of these specific decay chains. In addition, phenomenological MSSM (pMSSM) [31] models are used to study the sensitivity to realistic scenarios where more complex decay chains are present alongside the simpler ones.

Searches for direct $\tilde{t}_{1}$ pair production have previously been reported by the ATLAS [3238] and CMS [39-43] collaborations, as well as by the CDF and D $\varnothing$ collaborations (for example refs. [44, 45]) and the LEP collaborations [46]. Indirect searches for stops, mediated by gluino pair production, have been reported by the ATLAS [47-50] and CMS [39, 40, 5155] collaborations.

\section{Analysis strategy}

Searching for $\tilde{t}_{1}$ pair production in the various decay modes and over a wide range of stop masses requires different analysis approaches. The $\tilde{t}_{1}$ pair production cross-section falls

\footnotetext{
${ }^{3}$ Electrons and muons from $\tau$ decays are included.
} 


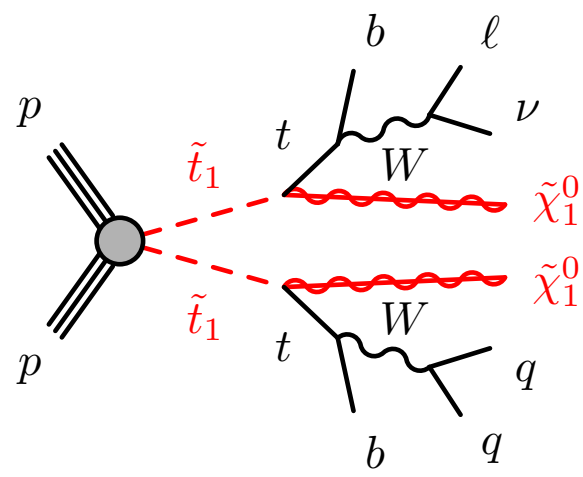

(a)

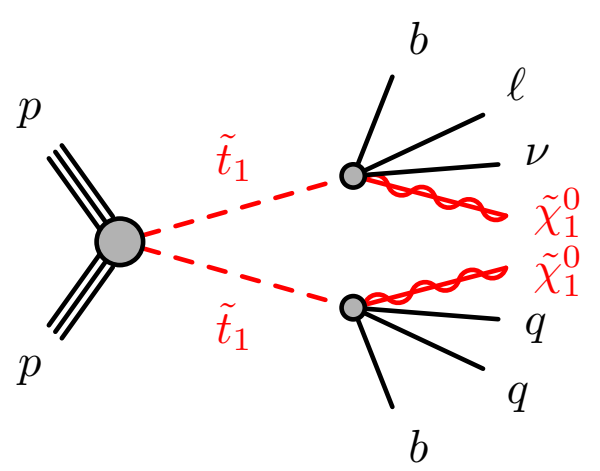

(c)

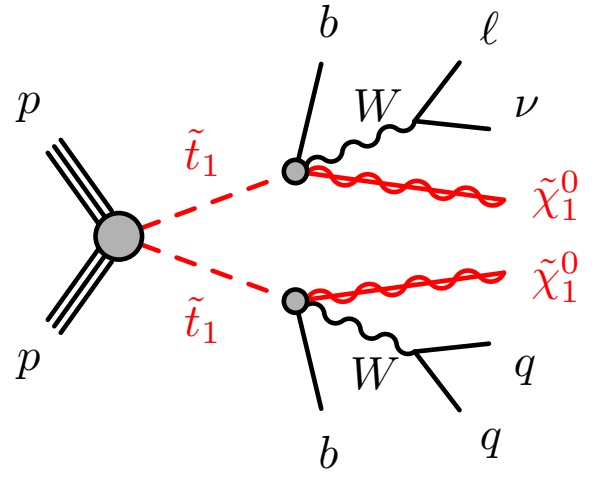

(b)

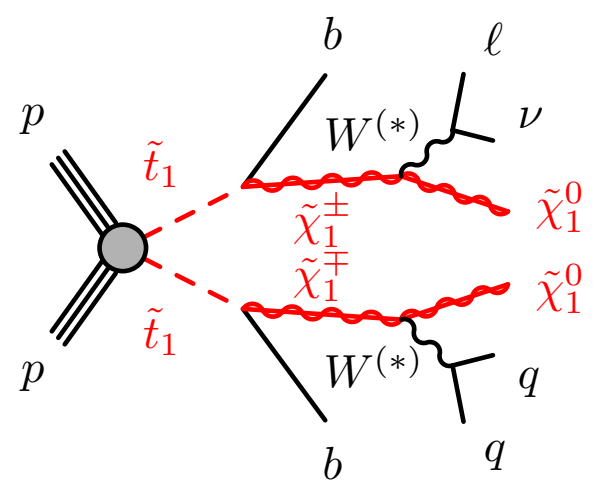

(d)

Figure 2. Diagrams illustrating the considered signal scenarios, which are referred to as (a) $\tilde{t}_{1} \rightarrow t \tilde{\chi}_{1}^{0}$, (b) $\tilde{t}_{1} \rightarrow b W \tilde{\chi}_{1}^{0}$ (three-body), (c) $\tilde{t}_{1} \rightarrow b f f^{\prime} \tilde{\chi}_{1}^{0}$ (four-body), (d) $\tilde{t}_{1} \rightarrow b \tilde{\chi}_{1}^{ \pm}$. Furthermore, a non-symmetric decay mode where each $\tilde{t}_{1}$ can decay via either $\tilde{t}_{1} \rightarrow t \tilde{\chi}_{1}^{0}$ or $\tilde{t}_{1} \rightarrow b \tilde{\chi}_{1}^{ \pm}$is considered (not shown). In these diagrams, the charge-conjugate symbols are omitted for simplicity; all scenarios begin with a top squark-antisquark pair. The three-body and four-body decays are assumed to proceed through an off-shell top quark, and an off-shell top quark followed by an off-shell $W$ boson, respectively.

rapidly with increasing stop mass $m_{\tilde{t}_{1}}$ : for the range targeted by this search, $m_{\tilde{t}_{1}} \sim 100$ $700 \mathrm{GeV}$, the cross-section at $\sqrt{s}=8 \mathrm{TeV}$ proton-proton $(p p)$ collisions decreases from $560 \mathrm{pb}$ to $8 \mathrm{fb}$. While the various $\tilde{t}_{1}$ decay modes considered all have identical final state objects - one electron or muon accompanied by one neutrino (or more for a leptonic $\tau$ decay), two jets originating from bottom quarks (b-jets), two light-flavour jets, and two LSPs - their kinematic properties change significantly for the different decay modes and as a function of the masses of the stop, LSP, and lightest chargino (if present). The search presented in this paper is based on 15 dedicated analyses that target the various scenarios. The identification of $b$-jets ( $b$-tagging) is utilised in the event selections and for constructing kinematic variables. The search for a heavy stop exploits a specialised technique, which reconstructs several decay products in a single large-radius (large- $R$ ) jet. Low-momentum leptons (referred to as soft leptons) are reconstructed and identified to 
enhance the sensitivity for $\tilde{t}_{1} \rightarrow b \tilde{\chi}_{1}^{ \pm}$decays where the $\tilde{\chi}_{1}^{0}$ and $\tilde{\chi}_{1}^{ \pm}$states are close in mass. These and other tools and variables to discriminate signal from background, described in section 6, are used to design sets of requirements for the event selection. Each of these sets of requirements is referred to as a signal region (SR), and is optimised to target one or more signal scenarios. Furthermore, two different analysis techniques are employed, which are referred to as 'cut-and-count' and 'shape-fit'. The former is based on counting events in a single region of phase space (bin), while the latter employs several bins. By utilising different signal-to-background ratios in the various bins, shape-fits enhance the search sensitivity in challenging scenarios, where it is particularly difficult to separate signal from background. All SRs are described in section 7.

The dominant background in most SRs arises from top quark pair production $(t \bar{t})$ where both $W$ bosons decay leptonically (dileptonic $t \bar{t}$ ) but one of the leptons is not identified, is outside the detector acceptance, or is a hadronically decaying $\tau$ lepton. The sub-leading background for most SRs stems from $W+$ jets production. As part of each analysis, the $t \bar{t}$ and $W+$ jets backgrounds are estimated using dedicated control regions (CRs), making the analysis more robust against potential mis-modelling effects in simulated events and reducing the uncertainties on the background estimates. Other small backgrounds are estimated using simulation only. Dedicated samples are used to validate the background predictions. The background estimation including the definition of all CRs is detailed in section 8 .

The analysis results are based on maximum likelihood fits, which include the CRs to simultaneously normalise the $t \bar{t}$ and $W+$ jets backgrounds. Systematic uncertainties due to theoretical and experimental effects are considered for all background and signal processes, and are described in section 9. The final results and interpretations, both in terms of model-dependent exclusion limits on the masses of relevant SUSY particles and modelindependent upper limits on the number of beyond-SM events, are presented in section 10.

\section{The ATLAS detector}

The ATLAS experiment [56] is a multi-purpose particle physics detector with nearly $4 \pi$ steradian coverage in solid angle. It consists of an inner detector of tracking devices surrounded by a thin superconducting solenoid, electromagnetic and hadronic calorimeters, and a muon spectrometer in a toroidal magnetic field. The inner detector, in combination with the $2 \mathrm{~T}$ axial field from the solenoid, provides precision tracking and momentum measurement of charged particles up to $|\eta|=2.5$ and allows efficient $b$-jet identification. ${ }^{4}$ It consists of a silicon pixel detector, a semiconductor microstrip detector and a straw-tube tracker which also provides transition radiation measurements for electron identification. High-granularity liquid-argon (LAr) sampling electromagnetic calorimeters cover the pseudorapidity range $|\eta|<3.2$. The hadronic calorimeter system is based on two different tech-

\footnotetext{
${ }^{4}$ ATLAS uses a right-handed coordinate system with its origin at the nominal interaction point in the centre of the detector and the $z$-axis along the beam pipe. Cylindrical coordinates $(r, \phi)$ are used in the transverse plane, $\phi$ being the azimuthal angle around the beam pipe. The pseudorapidity $\eta$ is defined in terms of the polar angle $\theta$ by $\eta=-\ln \tan (\theta / 2)$, and the angular separation $\Delta R$ in the $\eta-\phi$ space is defined as $\Delta R=\sqrt{(\Delta \eta)^{2}+(\Delta \phi)^{2}}$.
} 
nologies, a scintillator-tile sampling calorimeter $(|\eta|<1.7)$ and a LAr sampling calorimeter $(1.5<|\eta|<3.2)$. LAr calorimeters in the most forward region $(3.1<|\eta|<4.9)$ provide electromagnetic and hadronic measurements. The muon spectrometer has separate trigger and high-precision tracking chambers, the former provide trigger coverage up to $|\eta|=2.4$ while the latter provide muon identification and momentum measurements for $|\eta|<2.7$. Events are selected by a three-level trigger system [57], the first level (L1) is implemented in customised hardware while the two high-level triggers (HLT) are software-based.

\section{Trigger and data collection}

The data used in this analysis were collected from March to December 2012 with the LHC operating at a $p p$ centre-of-mass energy of $\sqrt{s}=8 \mathrm{TeV}$. After application of beam, detector and data quality requirements, the total integrated luminosity is $20.3 \mathrm{fb}^{-1}$ with an uncertainty of $2.8 \%$. The uncertainty is derived, following the methodology detailed in ref. [58], from a preliminary calibration of the luminosity scale from beam-separation scans performed in November 2012.

The dataset was recorded using three different types of triggers based on requiring either an electron, a muon, or large $E_{\mathrm{T}}^{\text {miss }}$. The single-electron trigger identifies electrons based on the presence of an energy cluster in the electromagnetic calorimeter with a shower shape consistent with that of an electron, low hadronic leakage, and a matching track in the inner detector. The HLT threshold ${ }^{5}$ on the energy deposit transverse to the beam $\left(E_{\mathrm{T}}\right)$ is $24 \mathrm{GeV}$. An electron isolation criterion at the HLT requires the scalar sum of the transverse momenta $\left(p_{\mathrm{T}}\right)$ of tracks within a cone of radius $\Delta R=0.2$ around the electron (excluding the electron itself) to be less than $10 \%$ of the electron $E_{\mathrm{T}}$. The single-muon trigger identifies muons using tracks reconstructed in the muon spectrometer and inner detector. The $p_{\mathrm{T}}$ threshold at the HLT is $24 \mathrm{GeV}$. An isolation criterion at the HLT requires the scalar sum of the $p_{\mathrm{T}}$ of tracks within a cone of radius $\Delta R=0.2$ around the muon (excluding the muon itself) to be less than $12 \%$ of the muon $p_{\mathrm{T}}$. To recover some of the small efficiency loss for high- $p_{\mathrm{T}}$ leptons, events were also collected using complementary single-lepton triggers. These triggers have less stringent shower-shape requirements and no hadronic leakage criterion for electrons, and no isolation criteria, but have an increased $E_{\mathrm{T}}\left(p_{\mathrm{T}}\right)$ threshold of $60 \mathrm{GeV}(36 \mathrm{GeV})$ for electrons (muons). Corrections are applied to the simulated samples to account for small differences between data and simulation in the lepton trigger efficiencies.

The $E_{\mathrm{T}}^{\text {miss }}$ trigger is based on the vector sum of the transverse energies deposited in projective calorimeter trigger towers. A more refined calculation based on the vector sum of all calorimeter cells above noise is made at the HLT. The trigger $E_{\mathrm{T}}^{\mathrm{miss}}$ threshold at the HLT is $80 \mathrm{GeV}$, and it is fully efficient for offline-calibrated $E_{\mathrm{T}}^{\text {miss }}>150 \mathrm{GeV}$ in signal-like events. At the beginning of the 2012 data-taking, the $E_{\mathrm{T}}^{\text {miss }}$ trigger used in this analysis was disabled for the first three bunch crossings of every bunch train, causing a loss of $0.2 \mathrm{fb}^{-1}$ in integrated luminosity.

\footnotetext{
${ }^{5}$ The trigger thresholds refer to lower requirements on the given quantity, and the HLT thresholds are always more stringent than the corresponding L1 thresholds.
} 
Candidate events in the electron (muon) channel were collected using a logical-OR combination of the single-electron (single-muon) and $E_{\mathrm{T}}^{\text {miss }}$ triggers. Since the singlelepton trigger thresholds are too high for the soft-lepton selection, these candidate events were recorded using only the $E_{\mathrm{T}}^{\text {miss }}$ trigger. Consequently, the effective dataset for the soft-lepton selections amounts to an integrated luminosity of $20.1 \mathrm{fb}^{-1}$. All results quote a rounded value of $20 \mathrm{fb}^{-1}$, while inside the analysis the appropriate integrated luminosity values are used. The efficiency of the $E_{\mathrm{T}}^{\text {miss }}$ and lepton triggers are measured with $W \rightarrow \mu \nu$ and $Z \rightarrow \ell \ell$ data samples, respectively. In all cases the combined trigger efficiency is greater than $98 \%$ for simulated signal events satisfying the selection criteria for the analyses described in sections $7-8$.

\section{Simulated samples}

Samples of Monte Carlo (MC) simulated events are used for the description of the background and to model the SUSY signals. As detailed below, the samples are generated with either POWHEG-R2129 [59], ACERMC-3.8 [60], MADGRAPH-5.1.4.8 [61], SHERPA1.4.1 [62] or Herwig ++ 2.5.2 [63]. All POWHEG and SHERPA samples use the next-to-leading-order (NLO) parton distribution function (PDF) set CT10 [64], while samples generated with ACERMC, MADGRAPH or HERwiG++ use the CTEQ6L1 [65] PDF set. The ATLAS Underlying Event Tune 2B [66] is used for all MADGraph samples, while the samples generated with POWHEG or ACERMC use the PERUGiA 2011C [67] tune and samples generated with HERWIG++ use UEEE3 [68]. The SHERPA generator has an integrated underlying event tune. The fragmentation and hadronisation for the POWHEG, ACERMC, and MADGRAPH samples is performed with PYTHIA-6.426 [69], while SHERPA and HERWIG++ use their own built-in models.

The samples are processed with either a detector simulation [70] using GEANT4 [71] or a fast simulation framework where the showers in the electromagnetic and hadronic calorimeters are simulated using a parameterised description [72] and GEANT4 is used for the rest of the detector. The fast simulation has been validated against full GEANT4 simulation for several signal models and for the main background, $t \bar{t}$ production. All samples are produced with varying numbers of simulated minimum-bias interactions, generated with PYTHIA-8.160 [73], overlaid on the hard-scattering event to account for multiple $p p$ interactions in the same or nearby bunch crossings (pileup). The average number of interactions per bunch crossing is reweighted to match the distribution in data that varies between approximately 10 and 30 .

\subsection{Background samples}

A sample of $t \bar{t}$ events is generated with POWHEG using a top quark mass $m_{t}=172.5 \mathrm{GeV}$. The same top quark mass is used when simulating other signal or background processes involving top quarks. To account for discrepancies between data and simulated $t \bar{t}$ events, the simulated sample is reweighted as a function of the $p_{\mathrm{T}}$ of the $t \bar{t}$ system; the weights are based on the ATLAS measurement of the differential $t \bar{t}$ cross-section at $7 \mathrm{TeV}$, following the method described in ref. [74]. Single top quark production in the $s$-channel and the 
$W t$ mode are also generated with POWHEG, while the $t$-channel process is generated with ACERMC. In $W t$ production, the interference with $t \bar{t}$ at NLO in quantum chromodynamics (QCD) is treated by the diagram removal scheme [75]. Associated production of $t \bar{t}$ and vector bosons $(W, Z$ and $W W$ ) as well as single top production in association with a $Z$ boson, are generated with MADGraph with up to two additional partons. Samples of $W+$ jets and $Z / \gamma^{*}+$ jets are produced with SHERPA, containing up to four additional partons and the correct treatment of bottom and charm quark masses. The diboson processes $(W W, Z Z$ and $W Z)$ are also generated with SHERPA.

The processes are normalised using theoretical inclusive cross-sections, including higher-order QCD corrections where available. The $t \bar{t}$ production cross-section is calculated at next-to-next-to-leading order (NNLO) in QCD including resummation of next-to-nextto-leading logarithmic (NNLL) soft gluon terms with top ++2.0 [76-81]. Cross-sections for single top quark production are calculated to approximate NLO+NNLL precision [82-84]. The production of $t \bar{t}$ in association with vector bosons is calculated at NLO [85, 86], while the production of a single top quark in association with a $Z$ boson is normalised to the LO cross-sections from the generator, because NLO calculations are only available for $t$-channel production [87]. The cross-sections for the production of $W$ and $Z$ bosons are calculated with DYNNLO [88]. The production cross-sections for electroweak diboson production are calculated at NLO with MCFM [89, 90]. The $t \bar{t}$, single top, $W, Z$, and diboson calculations use the MSTW2008 NNLO PDF set [91], while the cross-sections for $t \bar{t}$ in association with a vector boson use the MSTW2008 NLO $(W)$ or CTEQ6.6M [92] $(Z)$ PDF set. The cross-sections for $t \bar{t}$ and $W$ production are used for the optimisation of the selections, while for the final results the two processes are normalised to data in control regions.

\subsection{Signal samples}

Signal samples of top squark-antisquark pairs are generated with different stop decay and mass configurations. The first scenario assumes the $\tilde{t}_{1} \rightarrow t \tilde{\chi}_{1}^{0}$ decay with a branching ratio $(\mathcal{B R})$ of $100 \%$. The samples are generated with HeRwIG ++ in a grid across the plane of $\tilde{t}_{1}$ and $\tilde{\chi}_{1}^{0}$ masses with a spacing of $50 \mathrm{GeV}$ for most of the plane; the grid is more finely sampled towards the diagonal region where $m_{\tilde{t}_{1}}$ approaches $m_{t}+m_{\tilde{\chi}_{1}^{0}}$. The $\tilde{t}_{1}$ is chosen to be mostly the partner of the right-handed top quark ${ }^{6}$ and the $\tilde{\chi}_{1}^{0}$ to be almost pure bino. This choice is consistent with a large $\mathcal{B R}$ for the given $\tilde{t}_{1}$ decay. Different hypotheses on the left/right mixing in the stop sector and the nature of the neutralino lead to different acceptance values. The acceptance is affected because the polarisation of the top quark changes as a function of the field content of the supersymmetric particles, which impacts the boost of the lepton in the top quark decay. A subset of models where the $\tilde{t}_{1}$ is purely $\tilde{t}_{\mathrm{L}}$ are studied to quantify this effect.

The second signal scenario assumes the $\tilde{t}_{1} \rightarrow b \tilde{\chi}_{1}^{ \pm} \rightarrow b W^{(*)} \tilde{\chi}_{1}^{0}$ decay with a $\mathcal{B R}$ of $100 \% .^{7}$ The stop pairs are always generated with MADGrAPH, while for the $\tilde{t}_{1}$ decay either MADGRAPH or PYTHIA is employed. For models where the $W$ boson is on-shell,

\footnotetext{
${ }^{6}$ The $\tilde{t}_{\mathrm{R}}$ component is given by the the off-diagonal entry of the stop mixing matrix. Here, this matrix is set with (off-) diagonal entries of approximately $( \pm 0.83) 0.55$.

${ }^{7}$ All possible decays of the (possibly virtual) $W$ boson are considered.
} 
the full $\tilde{t}_{1}$ decay is performed by MADGRAPH, while PYTHIA is used to decay the $\tilde{t}_{1}$ in models where the $W$ is off-shell. In the latter case, generating the full event with MADGRAPH would be computationally too expensive. Seven two-dimensional planes are defined to probe the three-dimensional parameter space of the $\tilde{t}_{1}, \tilde{\chi}_{1}^{ \pm}$, and $\tilde{\chi}_{1}^{0}$ masses. The typical grid spacing is $50 \mathrm{GeV}$; higher grid densities are generated in regions where a rapid change of sensitivity is expected. The boundary conditions are derived from the LEP chargino mass limit of $103.5 \mathrm{GeV}$ [30], and by requiring the $\tilde{\chi}_{1}^{ \pm}$mass to be below the $\tilde{t}_{1}$ mass. Six out of the seven planes span the $\tilde{t}_{1}$ and $\tilde{\chi}_{1}^{0}$ masses. The first plane sets the chargino mass to twice the LSP mass $\left(m_{\tilde{\chi}_{1}^{ \pm}}=2 m_{\tilde{\chi}_{1}^{0}}\right)$, motivated by the pattern in GUT-scale models with gaugino universality. The second and third planes fix the chargino mass to be above $\left(m_{\tilde{\chi}_{1}^{ \pm}}=150 \mathrm{GeV}\right)$ or close to $\left(m_{\tilde{\chi}_{1}^{ \pm}}=106 \mathrm{GeV}\right)$ the chargino mass limit, respectively. In the fourth and fifth planes the chargino mass and neutralino mass are relatively close, $m_{\tilde{\chi}_{1}^{ \pm}}-m_{\tilde{\chi}_{1}^{0}}=5 \mathrm{GeV}$ and $m_{\tilde{\chi}_{1}^{ \pm}}-m_{\tilde{\chi}_{1}^{0}}=20 \mathrm{GeV}$, respectively; small mass differences are motivated by higgsino-like states. The sixth plane sets the chargino mass to be slightly below the stop mass, $m_{\tilde{\chi}_{1}^{ \pm}}=m_{\tilde{t}_{1}}-10 \mathrm{GeV}$. The last plane fixes the stop mass, $m_{\tilde{t}_{1}}=300 \mathrm{GeV}$, while varying the $\tilde{\chi}_{1}^{ \pm}$and $\tilde{\chi}_{1}^{0}$ masses. The samples in all planes assume that the $\tilde{t}_{1}$ is a $\tilde{t}_{\mathrm{L}}$ state. The $\tilde{t}_{1} \rightarrow b \tilde{\chi}_{1}^{ \pm}$branching ratio might not reach $100 \%$ in the MSSM if the $\tilde{t}_{1} \rightarrow t+\tilde{\chi}_{1}^{0} / \tilde{\chi}_{2}^{0}$ decays are kinematically allowed, but high branching ratios can occur in the allowed parameter space, such as for the above choices of particle field content.

The $\mathcal{B R}=100 \%$ assumption is relaxed in a third signal scenario where a stop can decay either via $\tilde{t}_{1} \rightarrow t \tilde{\chi}_{1}^{0}$ or via $\tilde{t}_{1} \rightarrow b \tilde{\chi}_{1}^{ \pm}$. For this purpose, 'asymmetric' samples are generated where in each event one stop is forced to decay via one and the second stop via the other decay mode. The signal plane as a function of the $\mathcal{B R}$ can be probed by combining, with appropriate reweighting, the asymmetric samples with the two $\mathcal{B R}=100 \%$ samples for the $\tilde{t}_{1} \rightarrow t \tilde{\chi}_{1}^{0}$ and $\tilde{t}_{1} \rightarrow b \tilde{\chi}_{1}^{ \pm}$decays. The asymmetric samples are generated with the same generator settings used for the other $\tilde{t}_{1} \rightarrow b \tilde{\chi}_{1}^{ \pm}$samples, except for using the maximum stop mixing angle (yielding equal components of $\tilde{t}_{\mathrm{L}}$ and $\tilde{t}_{\mathrm{R}}$ ) since the stop mixing is directly related to the $\mathcal{B R}$. The mass points generated are identical to those for the $m_{\tilde{\chi}_{1}^{ \pm}}=2 m_{\tilde{\chi}_{1}^{0}}$ scenario.

The three- and four-body stop decay modes, $\tilde{t}_{1} \rightarrow b W \tilde{\chi}_{1}^{0}$ and $\tilde{t}_{1} \rightarrow b f f^{\prime} \tilde{\chi}_{1}^{0}$ respectively, are relevant for a relatively light stop, as shown in figure 1. Samples for each scenario are generated with the assumption of $\mathcal{B R}=100 \%$. The three-body samples are produced with HeRwiG ++ , which performs the full matrix element calculation of the three-body decay, using the same settings as for the $\tilde{t}_{1} \rightarrow t \tilde{\chi}_{1}^{0}$ decay mode. The four-body decay mode is generated with MADGRAPH interfaced with PYTHIA for the $\tilde{t}_{1}$ decay and for parton showering, and with up to one additional parton. The four-body decay itself is forced to proceed via a virtual $W$ boson. The $\tilde{t}_{1}$ and $\tilde{\chi}_{1}^{0}$ mass parameters are varied with a grid spacing between 25 and $50 \mathrm{GeV}$.

Signal cross-sections are calculated in the MSSM at NLO, including the resummation of soft gluon emission at next-to-leading-logarithmic accuracy (NLO+NLL) [93-95]. The nominal cross-section and the uncertainty are taken from an envelope of cross-section predictions using different PDF sets and factorisation and renormalisation scales, as de- 
scribed in ref. [96]. The $\tilde{t}_{1}$ pair production cross-section obtained using this prescription is $(5.6 \pm 0.8) \mathrm{pb}$ for $m_{\tilde{t}_{1}}=250 \mathrm{GeV}$, and $(0.025 \pm 0.004) \mathrm{pb}$ for $m_{\tilde{t}_{1}}=600 \mathrm{GeV}$.

Although the simplified models described above can probe large regions of the allowed SUSY parameter space, more realistic SUSY models can feature more complex stop decays involving the heavier charginos and neutralinos. To study the sensitivity of the various analyses to these well-motivated scenarios, the pMSSM models described in ref. [97] are used. These models produce a Higgs boson in the mass range $\left(m_{h}=126 \pm 3 \mathrm{GeV}\right)$, saturate the WMAP relic density [98] and produce values of fine-tuning no worse than 1 part in 100 using the measure proposed by Barbieri, Giudice and Ellis et al. [23, 99]. In all models the $\tilde{\chi}_{1}^{0}$ is the LSP. To investigate the impact of varying parameters other than the stop and LSP mass while at the same time avoiding the processing of a large number of events, only three different $\tilde{t}_{1}$ and $\tilde{\chi}_{1}^{0}$ mass regions are considered. Only models where both $\tilde{t}_{1} \rightarrow t \tilde{\chi}_{1}^{0}$ and $\tilde{t}_{1} \rightarrow b \tilde{\chi}_{1}^{ \pm}$are kinematically allowed are used. This serves to remove the models which have a branching ratio of $100 \%$ for only one decay mode, as these regions of parameter space are already probed by the simplified models. This results in a total of 27 models, for which top squark-antisquark pair events are generated with HERWIG ++ and processed with the fast simulation. Some details of the models are given in table 1. By keeping the stop and LSP masses fixed, the impact on the sensitivity from varying other parameters can be studied, such as the branching ratios to the heavier charginos and neutralinos. The sensitivity for pMSSM models can then be compared to that obtained in the simplified models with the corresponding stop and LSP masses.

\section{Physics object reconstruction and discriminating variables}

\subsection{Physics object reconstruction}

The reconstruction and identification of all final state objects used in this search, such as vertices, jets, leptons, and missing transverse momentum, is described in the following. Two sets of lepton identification criteria are utilised. One set defines the leptons used in the overlap removal procedure with jets and other objects and to veto events with more than one lepton. The second set imposes tighter identification criteria, and is used to select the primary lepton in the event.

The reconstructed primary vertex is required to be consistent with the beam diamond envelope and to have at least five associated tracks with $p_{\mathrm{T}}>0.4 \mathrm{GeV}$ [100]. If there are multiple primary vertices in an event, the vertex with the largest summed $p_{\mathrm{T}}^{2}$ of the associated tracks is chosen. Relevant quantities such as the track impact parameters are calculated with respect to the selected primary vertex.

Jets are reconstructed from three-dimensional noise-suppressed calorimeter energy clusters [101] using the anti- $k_{t}$ jet clustering algorithm $[102,103]$ with a radius parameter $(R)$ of 0.4 . The impact of pileup is statistically subtracted based on the jet area method [104]. To calibrate the reconstructed energy, jets are corrected for the effects of calorimeter response and inhomogeneities using energy- and $\eta$-dependent calibration factors based on simulation and validated with extensive test-beam and collision-data studies. In the simulation, this procedure calibrates the jet energies to those of the corresponding 


\begin{tabular}{|c|c|c|c|c|c|c|c|c|c|c|c|c|}
\hline \multicolumn{6}{|c|}{ Mass $[\mathrm{GeV}]$} & \multicolumn{5}{|c|}{ Branching ratio $\tilde{t}_{1} \rightarrow$} & \multirow[b]{2}{*}[T_{11}]{$^{2}$} & \multirow[b]{2}{*}[N_{11}]{$^{2}$} \\
\hline$\tilde{t}_{1}$ & $\tilde{\chi}_{1}^{0}$ & $\tilde{\chi}_{2}^{0}$ & $\tilde{\chi}_{3}^{0}$ & $\tilde{\chi}_{1}^{ \pm}$ & $\tilde{\chi}_{2}^{ \pm}$ & $t \tilde{\chi}_{1}^{0}$ & $t \tilde{\chi}_{2}^{0}$ & $t \tilde{\chi}_{3}^{0}$ & $b \tilde{\chi}_{1}^{ \pm}$ & $b \tilde{\chi}_{2}^{ \pm}$ & & \\
\hline 404 & 40 & 221 & 230 & 220 & 1073 & 0.09 & 0.01 & 0.09 & 0.81 & 0.00 & 0.53 & 0.96 \\
\hline 404 & 44 & 324 & 445 & 325 & 471 & 0.16 & 0.00 & 0.00 & 0.84 & 0.00 & 0.98 & 0.99 \\
\hline 407 & 46 & 368 & 372 & 367 & 1515 & 0.74 & 0.00 & 0.00 & 0.26 & 0.00 & 0.02 & 0.98 \\
\hline 408 & 49 & 187 & 207 & 188 & 376 & 0.02 & 0.31 & 0.23 & 0.41 & 0.04 & 0.97 & 0.95 \\
\hline 409 & 39 & 211 & 212 & 206 & 1768 & 0.05 & 0.24 & 0.02 & 0.68 & 0.00 & 0.56 & 0.95 \\
\hline 409 & 49 & 180 & 190 & 179 & 795 & 0.02 & 0.22 & 0.17 & 0.59 & 0.00 & 0.99 & 0.94 \\
\hline 410 & 40 & 232 & 253 & 234 & 427 & 0.11 & 0.25 & 0.00 & 0.64 & 0.00 & 0.96 & 0.97 \\
\hline 410 & 43 & 387 & 396 & 386 & 889 & 0.88 & 0.00 & 0.00 & 0.12 & 0.00 & 0.01 & 0.99 \\
\hline 413 & 42 & 197 & 367 & 197 & 385 & 0.03 & 0.10 & 0.00 & 0.85 & 0.02 & 0.95 & 0.98 \\
\hline 413 & 45 & 373 & 406 & 374 & 508 & 0.32 & 0.00 & 0.00 & 0.68 & 0.00 & 0.99 & 0.99 \\
\hline 414 & 45 & 194 & 440 & 195 & 453 & 0.03 & 0.14 & 0.00 & 0.83 & 0.00 & 0.96 & 0.99 \\
\hline 416 & 45 & 394 & 397 & 393 & 1975 & 0.90 & 0.00 & 0.00 & 0.10 & 0.00 & 0.99 & 0.99 \\
\hline 417 & 46 & 333 & 350 & 335 & 573 & 0.65 & 0.00 & 0.00 & 0.35 & 0.00 & 0.96 & 0.98 \\
\hline 418 & 39 & 206 & 209 & 202 & 1779 & 0.09 & 0.05 & 0.28 & 0.59 & 0.00 & 0.47 & 0.95 \\
\hline 546 & 46 & 292 & 310 & 292 & 520 & 0.02 & 0.28 & 0.24 & 0.44 & 0.01 & 0.98 & 0.98 \\
\hline 547 & 46 & 346 & 374 & 346 & 500 & 0.12 & 0.49 & 0.00 & 0.22 & 0.16 & 0.93 & 0.98 \\
\hline 550 & 40 & 225 & 235 & 225 & 760 & 0.02 & 0.28 & 0.24 & 0.46 & 0.00 & 0.98 & 0.96 \\
\hline 551 & 43 & 351 & 366 & 351 & 621 & 0.07 & 0.38 & 0.21 & 0.35 & 0.00 & 0.98 & 0.99 \\
\hline 552 & 41 & 249 & 275 & 252 & 420 & 0.02 & 0.20 & 0.21 & 0.44 & 0.13 & 0.98 & 0.97 \\
\hline 552 & 42 & 332 & 337 & 331 & 1496 & 0.05 & 0.47 & 0.35 & 0.13 & 0.00 & 0.99 & 0.98 \\
\hline 552 & 43 & 346 & 350 & 344 & 1501 & 0.08 & 0.27 & 0.52 & 0.13 & 0.00 & 0.97 & 0.98 \\
\hline 552 & 43 & 385 & 397 & 385 & 731 & 0.36 & 0.00 & 0.00 & 0.64 & 0.00 & 0.97 & 0.99 \\
\hline 554 & 44 & 439 & 445 & 439 & 1007 & 0.21 & 0.00 & 0.00 & 0.79 & 0.00 & 0.99 & 0.99 \\
\hline 555 & 47 & 279 & 287 & 280 & 933 & 0.04 & 0.54 & 0.38 & 0.04 & 0.00 & 0.97 & 0.97 \\
\hline 553 & 147 & 169 & 444 & 168 & 455 & 0.31 & 0.12 & 0.00 & 0.27 & 0.30 & 0.07 & 0.93 \\
\hline 554 & 151 & 195 & 207 & 191 & 1969 & 0.09 & 0.35 & 0.43 & 0.12 & 0.00 & 0.88 & 0.68 \\
\hline 546 & 154 & 210 & 213 & 200 & 434 & 0.07 & 0.40 & 0.34 & 0.05 & 0.14 & 0.86 & 0.70 \\
\hline
\end{tabular}

Table 1. Properties of the 27 selected pMSSM models. The table contains the masses of the stop, of neutralinos and of the charginos, the branching ratios of the stop decays, the $\tilde{t}_{\mathrm{L}}$ content of the $\tilde{t}_{1}\left(\left[T_{11}\right]^{2}\right.$, with $T$ being the stop mixing matrix) and the bino content of the $\chi_{1}^{0}\left(\left[N_{11}\right]^{2}\right.$, with $N$ being the neutralino mixing matrix). 
jets constructed from stable simulated particles (particle-level jets). In-situ measurements are used to further correct the data to match the energy scale in simulated events $[105,106]$. Events containing jets that are likely to have arisen from detector noise, cosmic-ray muons, or machine-induced backgrounds such as beam-gas interactions and beam-halo particles, are removed [106]. Only jets with $p_{\mathrm{T}}>20 \mathrm{GeV}$ are considered. After the overlap removal procedure (described below), jets are required to have $|\eta|<2.5$.

A second collection of anti- $k_{t}$ jets reconstructed with $R=1.0$ is used to collect collimated decay products of high- $p_{\mathrm{T}}$ top quarks and $W$ bosons; these jets are referred to as large- $R$ jets [107]. The energy calibration is based on the same strategy as used for the jets with $R=0.4$ [107]. Jet trimming [108] — a procedure to remove contributions from pileup and from the underlying event by discarding softer components of the jet - is applied with a $k_{t}$ sub-jet size $R_{\mathrm{sub}}=0.3$ and a transverse momentum of the subjet relative to the large- $R$ jet, $f_{\text {cut }}$, greater than 0.05 . Large- $R$ jets are required to have $p_{\mathrm{T}}>150 \mathrm{GeV}$ and $|\eta|<2.0$. The invariant mass of large- $R$ jets is obtained from the energy and momentum of the jet constituents (themselves treated as massless) after the trimming procedure. In addition to the energy calibration, a mass calibration is applied to both data and simulation that accounts for differences between the jet masses derived at particle- and reconstruction-level. Large- $R$ jets may overlap with other physics objects such as jets or leptons; no overlap removal procedure between large- $R$ jets and other objects is applied. Consequently, large- $R$ jets are neither an input to the calculation of the missing transverse momentum, nor considered for the identification of $b$-jets.

The identification of $b$-jets uses the 'MV1' $b$-tagging algorithm (defined in refs. [109, 110]), which is based on a neural network and exploits both impact parameter and secondary vertex information. It is trained to assign high weights to $b$-jets and low weights to jets originating from light-flavour quarks or gluons. Three working points are chosen to maximise the search sensitivity for the various selections. They correspond to an average $b$-tagging efficiency of $60 \%, 70 \%$ and $80 \%$ for $b$-jets with $p_{\mathrm{T}}>20 \mathrm{GeV}$ and $|\eta|<2.5$ in simulated $t \bar{t}$ events. For these three working points, the average rejection factors for light-quark or gluon jets are approximately 600,140 , and 25 in the same simulated $t \bar{t}$ events [111], respectively. In the simulated samples, the efficiency of identifying $b$-jets and the probability for mis-identifying (mis-tagging) jets from light-flavour quarks, gluons and charm quarks are corrected to match those found in data.

Electron candidates are reconstructed from energy clusters in the electromagnetic calorimeter matched to a track measured in the inner detector $[112,113]$. They are required to have $p_{\mathrm{T}}>10 \mathrm{GeV},|\eta|<2.47$, and to satisfy the 'loose' shower shape and track selection criteria (defined in ref. [114]). The energy is corrected in data to match simulation, while the reconstruction efficiency is scaled in simulated samples to match that observed in data. Muons are reconstructed and identified either as a combined track in the muon spectrometer and inner detector systems, or as an inner detector track matched with a muon spectrometer segment [115-117]. Candidate muons are required to have $p_{\mathrm{T}}>10 \mathrm{GeV}$ and $|\eta|<2.4$. Corrections are applied to the momentum and to the reconstruction efficiency in simulation to match the data. For the soft-lepton selections, the thresholds are lowered to $p_{\mathrm{T}}>7 \mathrm{GeV}$ (electrons) and $p_{\mathrm{T}}>6 \mathrm{GeV}$ (muons), and electron candidates are required to satisfy the 'medium' identification criteria (defined in ref. [114]). 
Potential ambiguities between overlapping candidate objects are resolved based on their angular separation. If an electron candidate and a non-b-tagged jet (using the $70 \%$ efficiency $b$-tagging working point) overlap within $\Delta R<0.2$ of each other, then the object is considered to be an electron and the jet is dropped. If an electron candidate and any jet overlap within $0.2<\Delta R<0.4$ of each other, or if an electron candidate and a $b$-tagged jet overlap within $\Delta R<0.2$ of each other, then the electron is dropped and the jet is retained. If a muon candidate and any jet overlap within $\Delta R<0.4$ of each other, then the muon is not considered and the jet is kept. For the analysis exploiting a large- $R$-jet, the last requirement is changed to $\Delta R<0.1$, still between the muon and the $R=0.4$ jets, to recover efficiency losses in boosted topologies. The remaining leptons are referred to as 'baseline' leptons, and are used to veto events with more than one lepton.

Photons are not used in the main selections in this analysis, but they are used to select events for one validation sample. Photon candidates must satisfy the 'tight' quality criteria with $p_{\mathrm{T}}>20 \mathrm{GeV}$ and $|\eta|<2.47[118,119]$. For the validation sample selection only, jets close to a photon, with $\Delta R<0.2$, are dropped.

An event-veto based on identifying hadronically decaying $\tau$ leptons $\left(\tau_{\text {had }}\right)$ is used in some selections to reject $t \bar{t}$ background. The $\tau_{\text {had }}$ candidates are reconstructed in the same way as jets with $p_{\mathrm{T}}>15 \mathrm{GeV}$ and $|\eta|<2.47$, but calibrated separately to account for a different calorimeter response. The $\tau$-identification is performed with a boosted decision tree (BDT) discriminator [120, 121], which combines tracking information and the transverse and longitudinal shapes of the energy deposits in the calorimeter. If a $\tau_{\text {had }}$ candidate overlaps with any baseline lepton within $\Delta R<0.2$, the $\tau_{\text {had }}$ is not counted.

The missing transverse momentum vector $\vec{p}_{\mathrm{T}}^{\text {miss }}$ is the negative vector sum of the $p_{\mathrm{T}}$ of reconstructed objects in the event: jets with $p_{\mathrm{T}}>20 \mathrm{GeV}$, charged lepton (electron and muon) and photon candidates with $p_{\mathrm{T}}>10 \mathrm{GeV}$, and calibrated calorimeter clusters not assigned to these physics objects [122, 123].

The lepton identification criteria are tightened for the selection of the primary electron or muon in the event. The lepton $p_{\mathrm{T}}$ is required to be above $25 \mathrm{GeV}$, except for the softlepton selections where the baseline thresholds of $7 \mathrm{GeV}$ (electron) or $6 \mathrm{GeV}$ (muon) are kept. Electrons are required to satisfy the 'tight' selection criteria (defined in ref. [114]), and are required to satisfy a track-isolation criterion. The scalar sum of the $p_{\mathrm{T}}$ of tracks associated with the primary vertex and found within a cone of radius $\Delta R=0.2$ around the electron (excluding the electron itself) is required to be less than $10 \%$ of the electron $p_{\mathrm{T}}$. Similarly, a muon isolation criterion is imposed: the track isolation is required to be less than $1.8 \mathrm{GeV}$ in a cone of radius $\Delta R=0.2$. A less stringent muon isolation criterion is used for the analysis using a large- $R$ jet: the track isolation is required to be less than $12 \%$ of the muon $p_{\mathrm{T}}$. This helps to recover signal efficiency losses in boosted topologies. For the analyses based on a soft lepton, the 'tight' electron selection is omitted (keeping the 'medium' criteria from the baseline selection), and a modified version of the track-isolation is applied to electrons and muons: the scalar sum of the $p_{\mathrm{T}}$ of tracks within a cone of radius $\Delta R=0.3$ around the lepton (excluding the lepton itself) is required to be less than $16 \%$ $(12 \%)$ of the electron (muon) $p_{\mathrm{T}}$. Furthermore, the impact parameters along the beam direction $\left(z_{0}\right)$ and in the transverse plane $\left(d_{0}\right)$ are used to impose additional soft-lepton 
requirements: $\left|z_{0} \sin \theta\right|<0.4(0.4) \mathrm{mm}$ and $\left|d_{0} / \sigma_{d_{0}}\right|<5(3)$ for electrons (muons), where $\sigma_{d_{0}}$ is the uncertainty on $d_{0}$. The modified criteria of the soft-lepton selection are specifically optimised to suppress low- $p_{\mathrm{T}}$ jets mis-identified as isolated leptons.

\subsection{Tools to discriminate signal from background}

Requiring one isolated lepton $(\ell)$, several jets, and $E_{\mathrm{T}}^{\text {miss }}$ selects a sample enriched in semileptonic $t \bar{t}$ and $W+$ jets events. Both backgrounds are reduced by requiring the transverse mass $\left(m_{\mathrm{T}}\right)$ to be above the $W$ boson mass, where $m_{\mathrm{T}}$ is defined by

$$
m_{\mathrm{T}}=\sqrt{2 \cdot p_{\mathrm{T}}^{\ell} \cdot E_{\mathrm{T}}^{\mathrm{miss}}\left(1-\cos \Delta \phi\left(\vec{\ell}, \vec{p}_{\mathrm{T}}^{\mathrm{miss}}\right)\right)}
$$

Here $p_{\mathrm{T}}^{\ell}$ is the lepton $p_{\mathrm{T}}$, and $\Delta \phi\left(\vec{\ell}, \vec{p}_{\mathrm{T}}^{\text {miss }}\right)$ is the azimuthal angle between the lepton and the $\vec{p}_{\mathrm{T}}^{\text {miss }}$ directions. ${ }^{8}$ The dominant background after this requirement stems from dileptonic $t \bar{t}$ events, where one lepton is not identified, or is outside the detector acceptance, or is a hadronically decaying $\tau$ lepton. In all of these cases, the $t \bar{t}$ decay products include two or more high- $p_{\mathrm{T}}$ neutrinos, resulting in large $E_{\mathrm{T}}^{\text {miss }}$ and large $m_{\mathrm{T}}$ values. Requiring one or more $b$-tagged jets further removes $W$ +jets events, while a $b$-tag veto reduces the $t \bar{t}$ background but also the stop signal in most models. All but one SR require at least one or two $b$-tagged jets.

A number of variables and tools have been developed specifically to suppress the different types of dileptonic $t \bar{t}$ events. The detailed definitions of the variables are provided in appendix A.

- $a m_{\mathrm{T} 2}$ and $m_{\mathrm{T} 2}^{\tau}$ are two variants of the variable $m_{\mathrm{T} 2}$ [124], which is a generalisation of the transverse mass applied to signatures with two particles that are not directly detected. Figure 3 illustrates the $t \bar{t}$ event topologies targeted by the two variables.

The first variant is a form of asymmetric $m_{\mathrm{T} 2}\left(a m_{\mathrm{T} 2}\right)[125-127]$ in which the undetected particle is the $W$ boson for the branch with the lost lepton and the neutrino is the missing particle for the branch with the observed charged lepton. For dileptonic $t \bar{t}$ events with a lost lepton, $a m_{\mathrm{T} 2}$ is bounded from above by the top quark mass, whereas new physics can exceed this bound.

The second $m_{\mathrm{T} 2}$ variant $\left(m_{\mathrm{T} 2}^{\tau}\right)$ is designed for events with a hadronically decaying $\tau$ lepton by using the ' $\tau$-jet' as a visible particle for one branch and the observed lepton for the other branch. For $t \bar{t}$ events where one $W$ boson decays leptonically and the other to a $\tau_{\text {had }}$, the endpoint is the $W$ boson mass in the limit of collinear neutrinos.

- topness is a variable designed to identify and suppress partially reconstructed dileptonic $t \bar{t}$ events, as proposed in ref. [128]. The topness variable is based on minimising a $\chi^{2}-$ type function indicating the similarity of the event to dileptonic $t \bar{t}$ events. Similar to the $a m_{\mathrm{T} 2}$ variable, one lepton is assumed to be lost.

- A hadronic top mass, $m_{\text {had-top }}$, is designed to reject dileptonic $t \bar{t}$ events while retaining signal events that contain a hadronically decaying on-shell top quark, as in the $\tilde{t}_{1} \rightarrow t \tilde{\chi}_{1}^{0}$

\footnotetext{
${ }^{8}$ This formula of $m_{\mathrm{T}}$ makes the assumption that the lepton mass is negligible.
} 

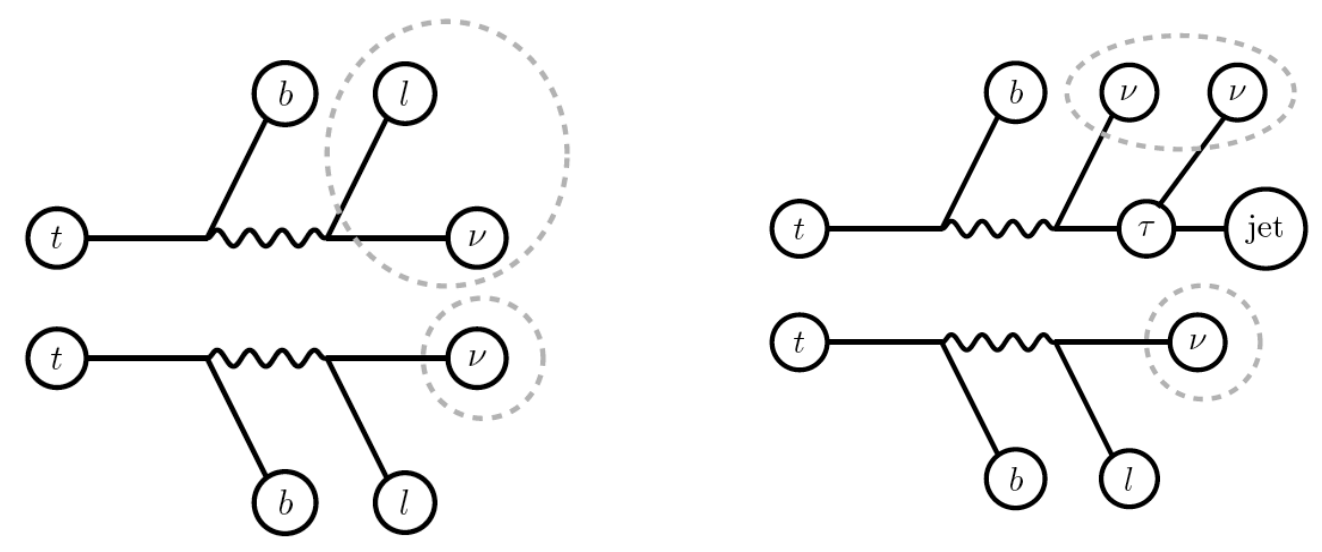

Figure 3. Illustration of the construction of the $a m_{\mathrm{T} 2}$ (left) and $m_{\mathrm{T} 2}^{\tau}$ (right) variables, which are used to discriminate against dileptonic $t \bar{t}$ background with one lost lepton (left) or with a hadronically decaying $\tau$ (right). The dashed lines indicate the objects that are assumed to be undetected ('lost') for the purpose of defining the two variables.

decay mode. The $m_{\text {had-top }}$ variable is a three-jet invariant mass, where the jets are selected by minimising a $\chi^{2}$-distribution taking into account the jet momenta and energy resolutions [105, 129].

- Dedicated $\tau$-identification criteria are used to reject $t \bar{t}$ events which contain a hadronic $\tau$ decay. For the construction of the $\tau$-veto, the reconstructed $\tau_{\text {had }}$ candidates, as previously defined, are subject to further selection requirements (described in appendix A). Three $\tau$-veto working points are defined: loose, tight, and extra-tight.

- A track-veto is designed to reject events which contain an isolated track not associated with a baseline lepton. This complements the second-lepton veto, and helps to reject $t \bar{t}$ events with a one-prong $\tau_{\text {had }}$. The selection criteria are detailed in appendix A.

Multijet events can pass the event selection if a jet is mis-identified as a lepton or when a real lepton from a heavy-flavour decay satisfies the isolation criteria, and if large $E_{\mathrm{T}}^{\text {miss }}$ occurs due to mis-measured jets. The former is suppressed by the lepton isolation criteria, while the following variables are used to reduce the latter effect.

- $\Delta \phi\left(\right.$ jet $\left._{i}, \vec{p}_{\mathrm{T}}^{\text {miss }}\right)$, the azimuthal opening angle between jet $i$ and $\vec{p}_{\mathrm{T}}^{\text {miss }}$, is used to suppress multijet events where $\vec{p}_{\mathrm{T}}^{\text {miss }}$ is aligned with a jet.

- $E_{\mathrm{T}}^{\text {miss }} / \sqrt{H_{\mathrm{T}}}$, where $H_{\mathrm{T}}$ is defined as the scalar $p_{\mathrm{T}}$ sum of the four leading jets, is an approximation of the $E_{\mathrm{T}}^{\text {miss }}$ significance.

- $E_{\mathrm{T}}^{\mathrm{miss}} / m_{\mathrm{eff}}$, where $m_{\mathrm{eff}}=H_{\mathrm{T}}+p_{\mathrm{T}}^{\ell}+E_{\mathrm{T}}^{\mathrm{miss}}$.

- $H_{\mathrm{T}, \text { sig }}^{\text {miss }}$ is an object-based missing transverse momentum, divided by the per-event resolution of the jets. The object-based missing transverse momentum is the negative sum of the jets and lepton vectors. A detailed description is given in appendix A. 


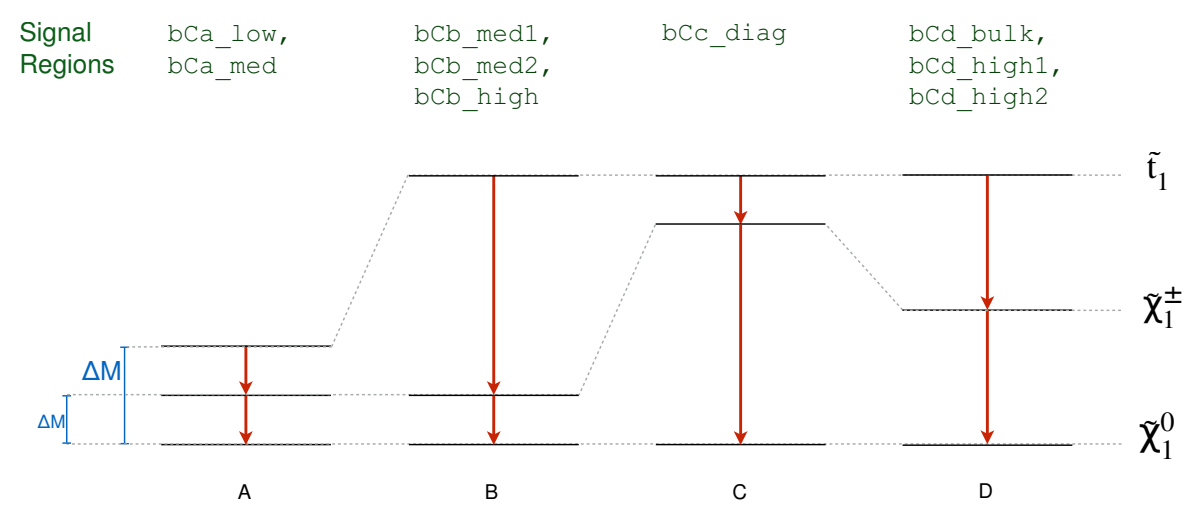

Figure 4. Schematic diagram of the mass hierarchies for the $\tilde{t}_{1} \rightarrow b \tilde{\chi}_{1}^{ \pm}$decay mode, with a subsequent $\tilde{\chi}_{1}^{ \pm} \rightarrow W^{(*)} \tilde{\chi}_{1}^{0}$ decay. A list of the corresponding signal regions is given above the diagram.

\section{Signal selections}

Signal selections are optimised using simulated samples only. The metric of the optimisation is to maximise the exclusion sensitivity for the various decay modes, and for different regions of SUSY simplified model parameter space. A set of signal benchmark models, selected to cover the various stop scenarios, was used for the optimisation considering all studied discriminating variables and including statistical and systematic uncertainties. The shape-fits employ multiple bins in one or two discriminating variables, which were selected considering the signal and background separation potential, inter-variable correlations, systematic uncertainties, and modelling of the data.

Table 2 summarises all 15 SRs with a brief description of the targeted signal scenarios, the exclusion analysis techniques, and forward references to the tables which list the event selection details. Four SRs target the $\tilde{t}_{1} \rightarrow t \tilde{\chi}_{1}^{0}$ decay. The corresponding SR labels begin with tN, which is an acronym for 'top neutralino'; additional text in the label describes the stop mass region, for example tN_diag targets the 'diagonal' of the $\tilde{t}_{1}-\tilde{\chi}_{1}^{0}$ mass plane. Nine SRs target the $\tilde{t}_{1} \rightarrow b \tilde{\chi}_{1}^{ \pm}$decay, where the SR labels follow the same logic: the first two characters bC stand for 'bottom chargino', a third letter (' $a$ ' to ' $d$ ') denotes the four different mass hierarchies illustrated in figure 4 , and the last piece of text describes the stop mass region. Furthermore, two SRs labelled 3body and tNbC_mix are dedicated to the three-body decay mode $\left(\tilde{t}_{1} \rightarrow b W \tilde{\chi}_{1}^{0}\right)$, and the mixed scenario where $\tilde{t}_{1} \rightarrow t \tilde{\chi}_{1}^{0}$ and $\tilde{t}_{1} \rightarrow b \tilde{\chi}_{1}^{ \pm}$decays both occur, respectively. The SRs are not mutually exclusive.

All SRs employ selection requirements to suppress the multijet background, and most SRs use the tools described in section 6.2 to reduce the dileptonic $t \bar{t}$ background. Shape-fit techniques are employed to derive model-dependent exclusion limits where useful, while for all model-independent results a simple cut-and-count approach is used. This procedure implies that for SRs using shape-fits one bin is probed at a time to extract the modelindependent results. Only a single bin, or the four bins with highest signal-to-background ratio are included; these are referred to as signal-sensitive bins. The model-dependent 


\begin{tabular}{|c|c|c|c|}
\hline SR & Signal scenario & Exclusion technique & Table \\
\hline tN_diag & $\tilde{t}_{1} \rightarrow t \tilde{\chi}_{1}^{0}, m_{\tilde{t}_{1}} \gtrsim m_{t}+m_{\tilde{\chi}_{1}^{0}}$ & shape-fit $\left(E_{\mathrm{T}}^{\text {miss }}\right.$ and $\left.m_{\mathrm{T}}\right)$ & 4 \\
\hline tN_med & $\tilde{t}_{1} \rightarrow t \tilde{\chi}_{1}^{0}, m_{\tilde{t}_{1}} \sim 550 \mathrm{GeV}, m_{\tilde{\chi}_{1}^{0}} \lesssim 225 \mathrm{GeV}$ & cut-and-count & 4 \\
\hline tN_high & $\tilde{t}_{1} \rightarrow t \tilde{\chi}_{1}^{0}, m_{\tilde{t}_{1}} \gtrsim 600 \mathrm{GeV}$ & cut-and-count & 4 \\
\hline tN_boost & $\tilde{t}_{1} \rightarrow t \tilde{\chi}_{1}^{0}, m_{\tilde{t}_{1}} \gtrsim 600 \mathrm{GeV}$, with a large- $R$ jet & cut-and-count & 4 \\
\hline bCa_low & $\tilde{t}_{1} \rightarrow b \tilde{\chi}_{1}^{ \pm}, \Delta M \lesssim 50 \mathrm{GeV}$ & shape-fit (lepton $p_{\mathrm{T}}$ ) & 5 \\
\hline & $\tilde{t}_{1} \rightarrow b f f^{\prime} \tilde{\chi}_{1}^{0}$ & & \\
\hline bCa_med & $\tilde{t}_{1} \rightarrow b \tilde{\chi}_{1}^{ \pm}, 50 \mathrm{GeV} \lesssim \Delta M \lesssim 80 \mathrm{GeV}$ & shape-fit (lepton $p_{\mathrm{T}}$ ) & 5 \\
\hline & $\tilde{t}_{1} \rightarrow b f f^{\prime} \tilde{\chi}_{1}^{0}$ & & \\
\hline bCb_med 1 & $\tilde{t}_{1} \rightarrow b \tilde{\chi}_{1}^{ \pm}, \Delta m \lesssim 25 \mathrm{GeV}, m_{\tilde{t}_{1}} \lesssim 500 \mathrm{GeV}$ & shape-fit $\left(a m_{\mathrm{T} 2}\right)$ & 5 \\
\hline bCb_high & $\tilde{t}_{1} \rightarrow b \tilde{\chi}_{1}^{ \pm}, \Delta m \lesssim 25 \mathrm{GeV}, m_{\tilde{t}_{1}} \gtrsim 500 \mathrm{GeV}$ & shape-fit $\left(a m_{\mathrm{T} 2}\right)$ & 5 \\
\hline $\mathrm{bCb} \_$med2 & $\tilde{t}_{1} \rightarrow b \tilde{\chi}_{1}^{ \pm}, \Delta m \lesssim 80 \mathrm{GeV}, m_{\tilde{t}_{1}} \lesssim 500 \mathrm{GeV}$ & shape-fit $\left(a m_{\mathrm{T} 2}\right.$ and $\left.m_{\mathrm{T}}\right)$ & 6 \\
\hline bCc_diag & $\tilde{t}_{1} \rightarrow b \tilde{\chi}_{1}^{ \pm}, m_{\tilde{t}_{1}} \gtrsim m_{\tilde{\chi}_{1}^{ \pm}}$ & cut-and-count & 6 \\
\hline bCd_bulk & $\tilde{t}_{1} \rightarrow b \tilde{\chi}_{1}^{ \pm},(\Delta M, \Delta m) \gtrsim 100 \mathrm{GeV}, m_{\tilde{t}_{1}} \lesssim 500 \mathrm{GeV}$ & shape-fit $\left(a m_{\mathrm{T} 2}\right.$ and $\left.m_{\mathrm{T}}\right)$ & 6 \\
\hline bCd_high1 & $\tilde{t}_{1} \rightarrow b \tilde{\chi}_{1}^{ \pm},(\Delta M, \Delta m) \gtrsim 100 \mathrm{GeV}, m_{\tilde{t}_{1}} \gtrsim 500 \mathrm{GeV}$ & cut-and-count & 6 \\
\hline bCd_high2 & $\tilde{t}_{1} \rightarrow b \tilde{\chi}_{1}^{ \pm}, \Delta M \gtrsim 250 \mathrm{GeV}, m_{\tilde{t}_{1}} \gtrsim 500 \mathrm{GeV}$ & cut-and-count & 6 \\
\hline 3body & $\tilde{t}_{1} \rightarrow b W \tilde{\chi}_{1}^{0}, m_{\tilde{t}_{1}} \lesssim 300 \mathrm{GeV}$ & shape-fit $\left(a m_{\mathrm{T} 2}\right.$ and $\left.m_{\mathrm{T}}\right)$ & 7 \\
\hline $\mathrm{tNbC} \_$mix & non-symmetric $\left(\tilde{t}_{1} \rightarrow t \tilde{\chi}_{1}^{0}, \tilde{t}_{1} \rightarrow b \tilde{\chi}_{1}^{ \pm}\right)$ & cut-and-count & 7 \\
\hline
\end{tabular}

Table 2. Overview of all signal regions (SRs) together with the targeted signal scenario, the analysis technique used for model-dependent exclusions, and a reference to the table with the event selection details. For the $\tilde{t}_{1} \rightarrow b \tilde{\chi}_{1}^{ \pm}$decay mode, the mass splittings $\Delta M=m\left(\tilde{t}_{1}\right)-m\left(\tilde{\chi}_{1}^{0}\right)$ and $\Delta m=m\left(\tilde{\chi}_{1}^{ \pm}\right)-m\left(\tilde{\chi}_{1}^{0}\right)$ are used to characterise the mass hierarchies, as illustrated in figure 4 . The SRs bCa_low, bCa_med, bCb_med1 and bCb_high employ selections based on a soft lepton.

and model-independent selections are defined in this section, and the corresponding fit configurations are described in section 10.

\subsection{Event preselection}

Common preselection criteria are employed as follows. Events are required to contain a reconstructed primary vertex. Furthermore, a set of quality requirements to avoid badly reconstructed jets, mismeasured- $E_{\mathrm{T}}^{\mathrm{miss}}$ and detector-related problems is imposed on all events. Events with a bad quality muon or with a cosmic-ray muon candidate ${ }^{9}$ are rejected.

Exactly one isolated lepton is required with $p_{\mathrm{T}}>25 \mathrm{GeV}$ except for the soft-lepton selections which employ a $p_{\mathrm{T}}>6(7) \mathrm{GeV}$ requirement for muons (electrons). The com-

\footnotetext{
${ }^{9}$ Defined as a muon candidate with a transverse or longitudinal impact parameter of $\left|d_{0}\right|>0.2 \mathrm{~mm}$ or $\left|z_{0}\right|>1 \mathrm{~mm}$.
} 


\begin{tabular}{|l|l|}
\hline Preselection & Description \\
\hline Trigger & $\begin{array}{l}\text { logical-OR combination of single-lepton and } E_{\mathrm{T}}^{\text {miss }} \\
\text { soft lepton: } E_{\mathrm{T}}^{\text {miss }} \text { trigger only. }\end{array}$ \\
\hline Data quality & jet and $E_{\mathrm{T}}^{\text {miss }}$ cleaning, cosmic-ray muon veto, primary vertex. \\
\hline Lepton & $\begin{array}{l}\text { one isolated electron or muon with } p_{\mathrm{T}}>25 \mathrm{GeV} ; \\
\text { soft lepton: the } p_{\mathrm{T}} \text { threshold is } 6(7) \mathrm{GeV} \text { for muons }(\text { electrons). }\end{array}$ \\
\hline $\mathbf{2}^{\text {nd }-l e p t o n ~ v e t o ~}$ & $\begin{array}{l}\text { No additional baseline lepton with } p_{\mathrm{T}}>10 \mathrm{GeV} ; \\
\text { soft lepton: no further baseline soft muon (electron) with } p_{\mathrm{T}}>6(7) \mathrm{GeV} .\end{array}$ \\
\hline Jets & The minimum jet multiplicity requirement varies between 2 and 4. \\
\hline $\boldsymbol{E}_{\mathbf{T}}^{\text {miss }}$ & $E_{\mathrm{T}}^{\text {miss }}>100 \mathrm{GeV}$ or tighter is required in all selections. \\
\hline
\end{tabular}

Table 3. Preselection criteria common to all signal selections.

mon lepton isolation criteria are tightened for the soft-lepton selections while they are relaxed for the analysis exploiting a large- $R$ jet (cf. section 6). Events containing additional baseline leptons are rejected. A minimum number of jets ranging between 2 and 4 , and $E_{\mathrm{T}}^{\text {miss }}>100 \mathrm{GeV}$ are common requirements amongst all analyses. Table 3 summarises the preselection criteria.

Figure 5 illustrates the separation power for a selection of discriminating variables. For these distributions, events are required to pass the preselection (table 3), to have at least four jets with $p_{\mathrm{T}}>25 \mathrm{GeV}$, one of which above $60 \mathrm{GeV}$, and with at least one of them $b$-tagged using the $70 \%$ working point, and to have $E_{\mathrm{T}}^{\text {miss }}>100 \mathrm{GeV}, m_{\mathrm{T}}>60 \mathrm{GeV}$ and $E_{\mathrm{T}}^{\text {miss }} / \sqrt{H_{\mathrm{T}}}>5 \mathrm{GeV}^{1 / 2}$. The $W+$ jets background is normalised to match data in a sample selected in the same way, except that a $b$-veto is imposed. The other processes are normalised to their theoretical cross-sections. Data and background estimation are seen to be in good agreement.

\subsection{Selections for the $\tilde{t}_{1} \rightarrow t \tilde{\chi}_{1}^{0}$ decay}

Stop pair production with subsequent $\tilde{t}_{1} \rightarrow t \tilde{\chi}_{1}^{0}$ decays leads to final state objects similar to that of $t \bar{t}$ production augmented by two $\tilde{\chi}_{1}^{0}$. Four SRs, labelled $t N \_d i a g, t N \_m e d, t N \_h i g h$, and $\mathrm{tN} \_$boost, target different regions in the $\tilde{t}_{1}-\tilde{\chi}_{1}^{0}$ mass plane and implement different analysis strategies. Table 4 details the event selections for these SRs. Criteria based on a subset of the variables outlined in section 6.2 , as well as optimised jet thresholds, a more stringent $E_{\mathrm{T}}^{\text {miss }}$ requirement, and a requirement on the angular separation between the highest- $p_{\mathrm{T}} b$-tagged jet and the lepton, $\Delta R(b$-jet, $\ell)$, are used to suppress $t \bar{t}$ and $W+$ jets backgrounds as well as to reduce the multijet background to a negligible level.

The loosest selection, tN_diag, employs a multi-binned shape-fit that targets the challenging parameter space where the stop and its decay products are nearly mass degenerate $\left(m_{\tilde{t}_{1}} \gtrsim m_{t}+m_{\tilde{\chi}_{1}^{0}}\right)$, also referred to as the 'diagonal'. The strategy of exploiting binned 


\begin{tabular}{|c|c|c|c|c|}
\hline & tN_diag & tN_med & tN_high & tN_boost \\
\hline Preselection & \multicolumn{4}{|c|}{ Default preselection criteria, cf. table 3 . } \\
\hline Lepton & \multicolumn{4}{|c|}{$=1$ lepton } \\
\hline Jets & $\begin{array}{l}\geq 4 \text { with } p_{\mathrm{T}}> \\
60,60,40,25 \mathrm{GeV}\end{array}$ & $\begin{array}{l}\geq 4 \text { with } p_{\mathrm{T}}> \\
80,60,40,25 \mathrm{GeV}\end{array}$ & $\begin{array}{l}\geq 4 \text { with } p_{\mathrm{T}}> \\
100,80,40,25 \mathrm{GeV}\end{array}$ & $\begin{array}{l}\geq 4 \text { with } p_{\mathrm{T}}> \\
75,65,40,25 \mathrm{GeV}\end{array}$ \\
\hline$b$-tagging & \multicolumn{4}{|c|}{$\geq 1 b$-tag (70\% eff.) amongst four selected jets } \\
\hline large- $R$ jet & \multicolumn{3}{|c|}{ - } & $\begin{array}{l}\geq 1, p_{\mathrm{T}}>270 \mathrm{GeV} \\
\text { and } m>75 \mathrm{GeV}\end{array}$ \\
\hline $\boldsymbol{\Delta} \phi\left(\right.$ jet $\left._{\mathbf{2}}^{\text {large- } \boldsymbol{R}}, \overrightarrow{\boldsymbol{p}}_{\mathrm{T}}^{\mathrm{miss}}\right)$ & \multicolumn{3}{|c|}{ - } & $>0.85$ \\
\hline$E_{\mathrm{T}}^{\mathrm{miss}}$ & $>100 \mathrm{GeV}$ & $>200 \mathrm{GeV}$ & $>320 \mathrm{GeV}$ & $>315 \mathrm{GeV}$ \\
\hline$m_{\mathrm{T}}$ & $>60 \mathrm{GeV}$ & $>140 \mathrm{GeV}$ & $>200 \mathrm{GeV}$ & $>175 \mathrm{GeV}$ \\
\hline$a m_{\mathrm{T} 2}$ & - & $>170 \mathrm{GeV}$ & $>170 \mathrm{GeV}$ & $>145 \mathrm{GeV}$ \\
\hline$m_{\mathrm{T} 2}^{\tau}$ & - & - & $>120 \mathrm{GeV}$ & - \\
\hline topness & - & - & - & $>7$ \\
\hline$m_{\text {had-top }}$ & $\in[130,205] \mathrm{GeV}$ & $\in[130,195] \mathrm{GeV}$ & $\in[130,250] \mathrm{GeV}$ & \\
\hline$\tau$-veto & tight & - & - & modified, see text. \\
\hline$\Delta R(b$-jet,$\ell)$ & $<2.5$ & - & $<3$ & $<2.6$ \\
\hline $\boldsymbol{E}_{\mathrm{T}}^{\mathrm{miss}} / \sqrt{\boldsymbol{H}_{\mathrm{T}}}$ & $>5 \mathrm{GeV}^{1 / 2}$ & & - & \\
\hline$H_{\mathrm{T}, \text { sig }}^{\text {miss }}$ & - & & 12.5 & $>10$ \\
\hline$\Delta \phi\left(\right.$ jet $\left._{i}, \vec{p}_{\mathrm{T}}^{\mathrm{miss}}\right)$ & $>0.8(i=1,2)$ & $>0.8(i=2)$ & - & $>0.5,0.3(i=1,2)$ \\
\hline \multicolumn{5}{|c|}{ Model-dependent selection: } \\
\hline & $\begin{array}{l}\text { shape-fit in } m_{\mathrm{T}} \text { and } \\
E_{\mathrm{T}}^{\mathrm{miss}} \text {, cf. figure } 6 .\end{array}$ & \multicolumn{3}{|c|}{ cut-and-count } \\
\hline \multicolumn{5}{|c|}{ Model-independent selection: } \\
\hline & $\begin{array}{l}\text { test } 4 \text { most } \\
\text { signal-sensitive } \\
\text { bins one-by-one. }\end{array}$ & \multicolumn{3}{|c|}{ cut-and-count } \\
\hline
\end{tabular}

Table 4. Selection criteria for SRs employed to search for $\tilde{t}_{1} \rightarrow t \tilde{\chi}_{1}^{0}$ decays. 

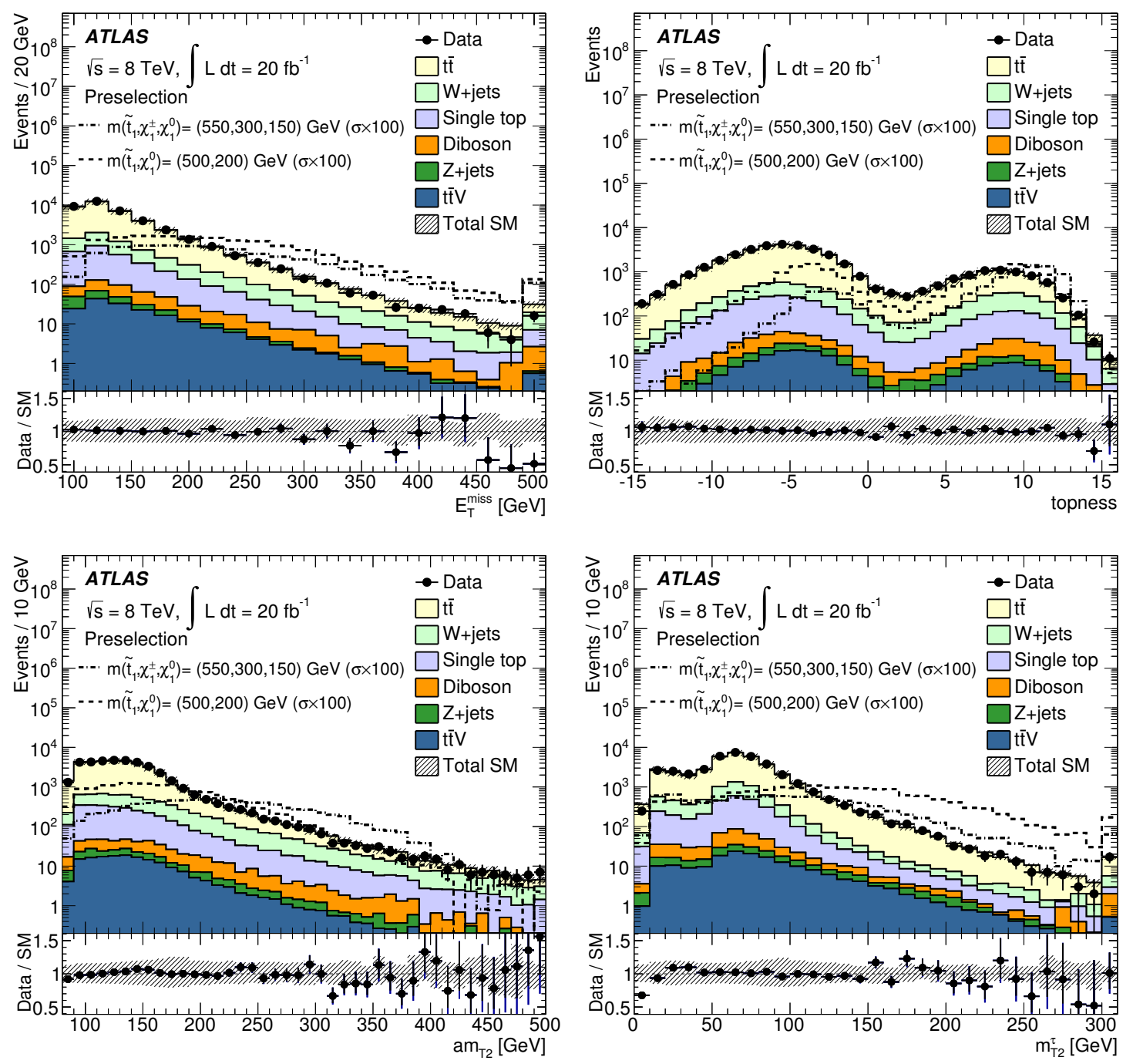

Figure 5. Comparison of data with estimated backgrounds in the $E_{\mathrm{T}}^{\mathrm{miss}}$ (top left), topness (top right), $a m_{\mathrm{T} 2}$ (bottom left), and $m_{\mathrm{T} 2}^{\tau}$ (bottom right) distributions for the preselection defined in the text. The uncertainty band includes statistical and all experimental systematic uncertainties. The last bin includes overflows. Benchmark signal models with cross-sections enhanced by a factor of 100 are overlaid for comparison.

shape information significantly improves the sensitivity. The two-dimensional shape-fit in the variables $m_{\mathrm{T}}$ and $E_{\mathrm{T}}^{\text {miss }}$ is illustrated in figure 6 (left plot). The top 12 bins serve both to probe a signal and to normalise the $t \bar{t}$ background; a subset of the 12 bins has a high purity in $t \bar{t}$ events. Three additional bins with a $b$-tag veto, shown in the bottom part, are used to derive the normalisation of the $W+$ jets background. The bins with $E_{\mathrm{T}}^{\text {miss }}>150 \mathrm{GeV}$ or $m_{\mathrm{T}}>140 \mathrm{GeV}$ are defined without upper boundaries.

The two SRs tN_med and tN_high target medium and high stop mass regions, respectively. Both SRs are based on a cut-and-count approach with relatively tight selections. The SR labelled tN_boost also targets models with a high stop mass and a nearly massless 

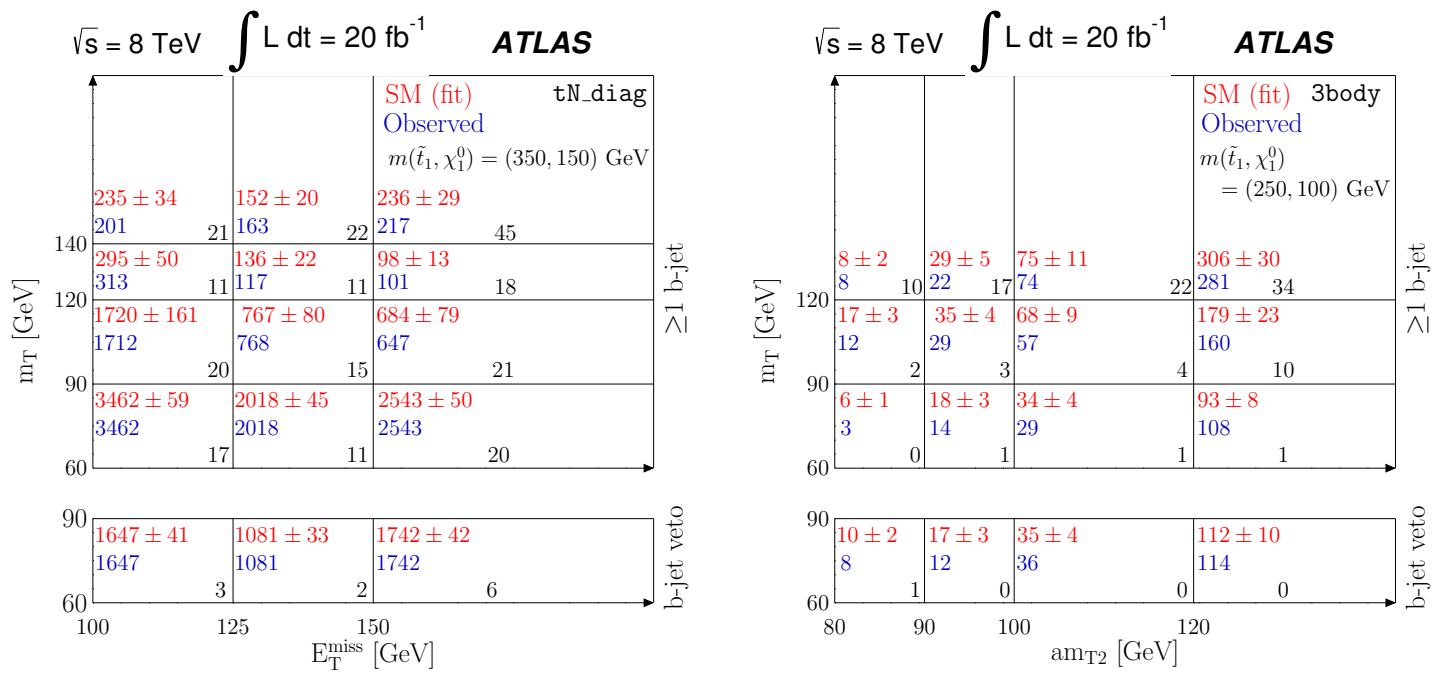

Figure 6. Schematic illustration of the tN_diag (left) and 3body (right) shape-fit binning. The $m_{\mathrm{T}}$ and $E_{\mathrm{T}}^{\mathrm{miss}}$ (left) or $a m_{\mathrm{T} 2}$ (right) variables are used to define a matrix of $4 \times 3$ bins (left) or $3 \times 4$ (right) in the top part, which is sensitive to stop models, while also being enriched with $t \bar{t}$ background. The bottom bins invert the $b$-tag requirement into a veto, and serve to normalise the $W+$ jets background. The numbers of observed events together with the estimated background, obtained using the background-only fit described in section 8 , are given for each bin. The data and estimated background are in perfect agreement in the six bottom bins for the left plot because the fit is configured to use these six bins together with six free parameters; the fit used for the right plot employs the bottom eight bins and two free parameters. For comparison the expected numbers of events for one signal model are shown.

LSP, but takes advantage of the 'boosted' topology. The selection assumes that either all decay products of the hadronically decaying top quark, or at least the decay products of the hadronically decaying $W$ boson, collimate into a jet with a radius of $\lesssim 1.0$. Figure 7 shows some of the relevant large- $R$ jet related distributions. The overlaid heavy stop benchmark model illustrates the separation power of the variables. The tN_boost selection requires at least one large- $R$ jet with $p_{\mathrm{T}}>270 \mathrm{GeV}$ and an invariant mass above $75 \mathrm{GeV}$. To further discriminate stop decays from the $t \bar{t}$ background, events with a second (ordered by $p_{\mathrm{T}}$ ) large- $R$ jet are required to have a minimum azimuthal distance between $\vec{p}_{\mathrm{T}}^{\text {miss }}$ and the second large- $R$ jet, $\Delta \phi\left(\right.$ jet $\left._{2}^{\text {large- } R}, \vec{p}_{\mathrm{T}}^{\text {miss }}\right)$. The extra-tight $\tau$-veto is applied to discard events with $\tau_{\text {had }}$ candidates well separated from large- $R$ jets, $\Delta R$ ( $\tau_{\text {had }}$, large- $R$-jet $)>2.6$, that satisfy the above $p_{\mathrm{T}}$ and mass requirements.

\subsection{Selections for the $\tilde{t}_{1} \rightarrow b \tilde{\chi}_{1}^{ \pm}$decay}

Nine SRs target scenarios where both stops decay as $\tilde{t}_{1} \rightarrow b \tilde{\chi}_{1}^{ \pm}$followed by subsequent $\tilde{\chi}_{1}^{ \pm} \rightarrow W^{(*)} \tilde{\chi}_{1}^{0}$ decays. The mass of the lightest chargino $m\left(\tilde{\chi}_{1}^{ \pm}\right)$relative to the $\tilde{t}_{1}$ and $\tilde{\chi}_{1}^{0}$ masses largely defines the kinematic properties. Figure 4 schematically illustrates the four distinct mass hierarchies, whose SRs are described below. 

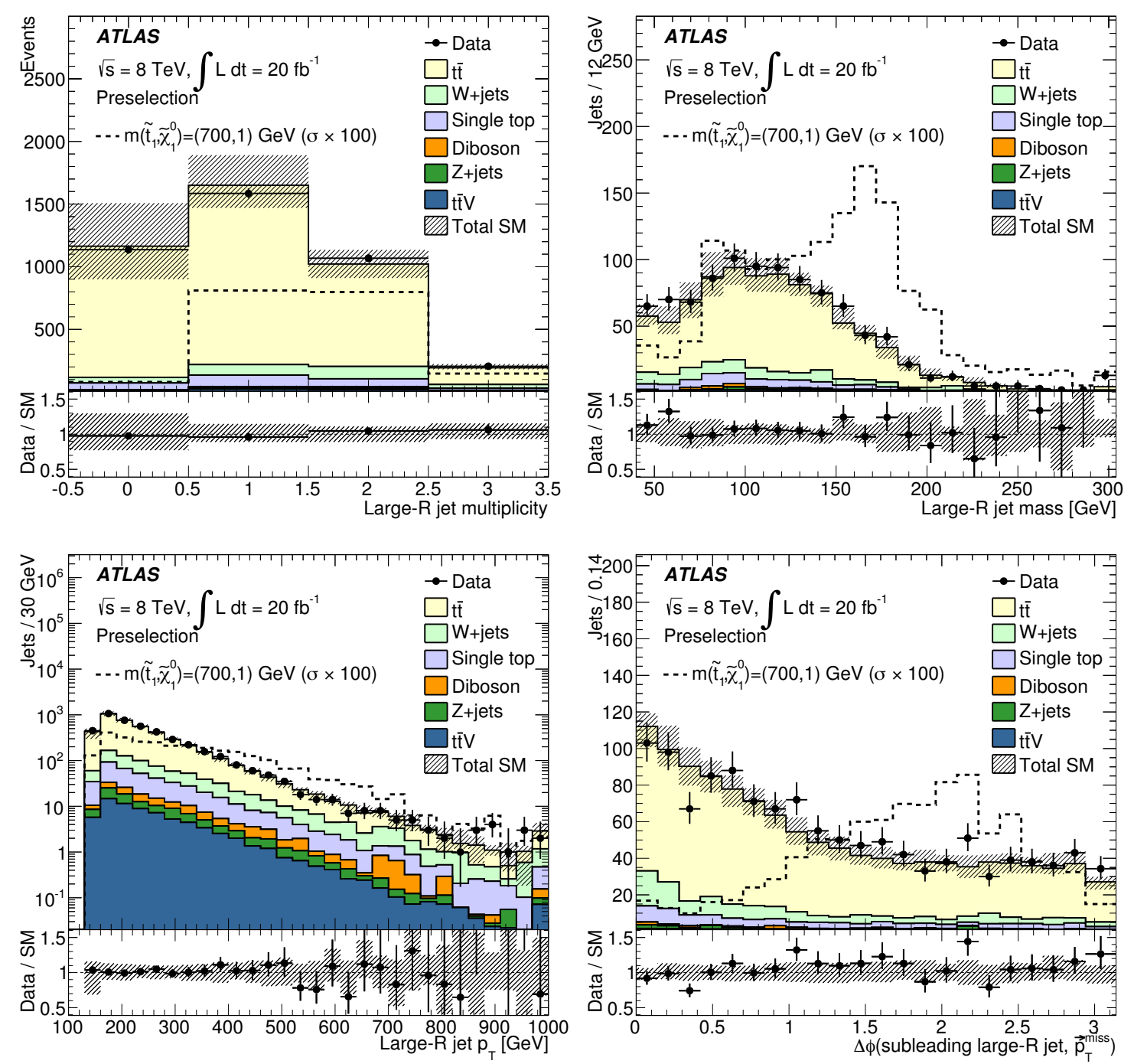

Figure 7. Comparison of data with background expectations of large- $R$ jet distributions: multiplicity (top left), invariant mass (top right), transverse momentum (bottom left), and distance in $\phi$ space between the second-highest- $p_{\mathrm{T}}$ large- $R$ jet and $\vec{p}_{\mathrm{T}}^{\text {miss }}$. Events are required to pass the preselection defined in section 7.1. In addition, the jet thresholds are tightened $\left(p_{\mathrm{T}}>75,65,40,25 \mathrm{GeV}\right)$ and a requirement of $m_{\mathrm{T}}>120 \mathrm{GeV}$ is imposed. The uncertainty band includes statistical and all experimental systematic uncertainties. The last bin includes overflows. A benchmark signal model, with cross-section enhanced by a factor of 100, is overlaid for comparison.

Selections for mass hierarchy (a): the selection of signal events with a small overall mass splitting, $\Delta M=m\left(\tilde{t}_{1}\right)-m\left(\tilde{\chi}_{1}^{0}\right)$, relies on the presence of an initial-state radiation (ISR) jet, against which the stop decay products recoil. Consequently, events with a hard leading jet are selected together with a soft lepton and relatively soft sub-leading jets. The leading jet must not satisfy the $b$-tagging criteria, while at least one $b$-tagged jet amongst the sub-leading jets is required. 


\begin{tabular}{|c|c|c|c|c|}
\hline & bCa_low & bCa_med & $\mathrm{bCb}$ med 1 & bCb_high \\
\hline Preselection & \multicolumn{4}{|c|}{ soft-lepton preselection, cf. table 3 . } \\
\hline Lepton & \multicolumn{2}{|c|}{$=1$ soft lepton } & \multicolumn{2}{|c|}{$=1$ soft lepton with $p_{\mathrm{T}}<25 \mathrm{GeV}$} \\
\hline Jets & $\begin{array}{l}\geq 2 \text { with } \\
p_{\mathrm{T}}>180,25 \mathrm{GeV}\end{array}$ & $\begin{array}{l}\geq 3 \text { with } \\
p_{\mathrm{T}}>180,25,25 \mathrm{GeV}\end{array}$ & \multicolumn{2}{|c|}{$\begin{array}{c}\geq 2 \text { with } \\
p_{\mathrm{T}}>60,60 \mathrm{GeV}\end{array}$} \\
\hline$\Delta \phi\left(\mathrm{jet}_{i}, \vec{p}_{\mathbf{T}}^{\text {miss }}\right)$ & \multicolumn{2}{|c|}{ - } & \multicolumn{2}{|c|}{$>0.4(\mathrm{i}=1,2)$} \\
\hline Jet veto & \multicolumn{2}{|c|}{ - } & $H_{\mathrm{T}, 2}<50 \mathrm{GeV}$ & - \\
\hline$b$-tagging & \multicolumn{2}{|c|}{$\geq 1$ sub-leading jet $b$-tagged ( $70 \%$ eff.) } & \multicolumn{2}{|c|}{ Leading two jets $b$-tagged ( $60 \%$ eff.) } \\
\hline$b$-veto & \multicolumn{2}{|c|}{$1^{\text {st }}$ jet not $b$-tagged ( $70 \%$ eff.) } & \multicolumn{2}{|c|}{ 一 } \\
\hline$m_{b b}$ & \multicolumn{2}{|c|}{ - } & \multicolumn{2}{|c|}{$>150 \mathrm{GeV}$} \\
\hline$E_{\mathrm{T}}^{\mathrm{miss}}$ & $>370 \mathrm{GeV}$ & $>300 \mathrm{GeV}$ & $>150 \mathrm{GeV}$ & $>250 \mathrm{GeV}$ \\
\hline$E_{\mathrm{T}}^{\mathrm{miss}} / \boldsymbol{m}_{\mathrm{eff}}$ & $>0.35$ & $>0.3$ & & - \\
\hline$m_{\mathrm{T}}$ & $>90 \mathrm{GeV}$ & $>100 \mathrm{GeV}$ & & - \\
\hline \multicolumn{5}{|c|}{ Model-dependent selection: shape-fit } \\
\hline & \multicolumn{2}{|c|}{4 bins in lepton $p_{\mathrm{T}}$ range $[6(7), 50] \mathrm{GeV}$} & \multicolumn{2}{|c|}{6 bins in $a m_{\mathrm{T} 2}$ range $[0,500] \mathrm{GeV}$} \\
\hline \multicolumn{5}{|c|}{ Model-independent selection: 1 bin with } \\
\hline & \multicolumn{2}{|c|}{ lepton $p_{\mathrm{T}}<25 \mathrm{GeV}$} & $a m_{\mathrm{T} 2}>170 \mathrm{Ge}$ & $a m_{\mathrm{T} 2}>200 \mathrm{GeV}$ \\
\hline
\end{tabular}

Table 5. Selection criteria for soft-lepton SRs, employed to search for $\tilde{t}_{1} \rightarrow b \tilde{\chi}_{1}^{ \pm}$decays. The two leftmost/rightmost SRs target mass hierarchies (a)/(b), illustrated in figure 4.

Two SRs, labelled bCa_low and bCa_med, are defined to probe scenarios with a mass splitting $\Delta M \lesssim 50 \mathrm{GeV}$, and $50 \mathrm{GeV} \lesssim \Delta M \lesssim 80 \mathrm{GeV}$, respectively. The SR event selections are listed in table 5 . The requirement of $\geq 3$ jets suppresses the $W+$ jets background in bCa_med. For bCa_low, the jet multiplicity requirement is lowered to $\geq 2$ to avoid large signal acceptance losses, but tighter $E_{\mathrm{T}}^{\text {miss }}$ and $E_{\mathrm{T}}^{\text {miss }} / m_{\text {eff }}$ thresholds are applied to keep the $W+$ jets and multijet backgrounds suppressed. Figure 8 compares data with estimated backgrounds in the lepton $p_{\mathrm{T}}$ and $E_{\mathrm{T}}^{\mathrm{miss}} / m_{\mathrm{eff}}$ distributions. The overlaid stop benchmark model motivates the selection of low- $p_{\mathrm{T}}$ leptons, and the background estimates show the non-negligible contribution from multijet events (with mis-identified leptons).

Model-dependent exclusion results are obtained using a shape-fit in the lepton $p_{\mathrm{T}}$ variable with four bins of approximately uniform widths in the range $[6(7), 50] \mathrm{GeV}$ for muons (electrons). For model-independent results, the cut-and-count approach is used with an additional lepton $p_{\mathrm{T}}<25 \mathrm{GeV}$ requirement.

Selections for mass hierarchy (b): signal scenarios with a moderately large $\Delta M$ but a small $\Delta m=m\left(\tilde{\chi}_{1}^{ \pm}\right)-m\left(\tilde{\chi}_{1}^{0}\right)$ feature two high- $p_{\mathrm{T}} b$-jets and low-momentum decay products from the two off-shell $W$ bosons. 

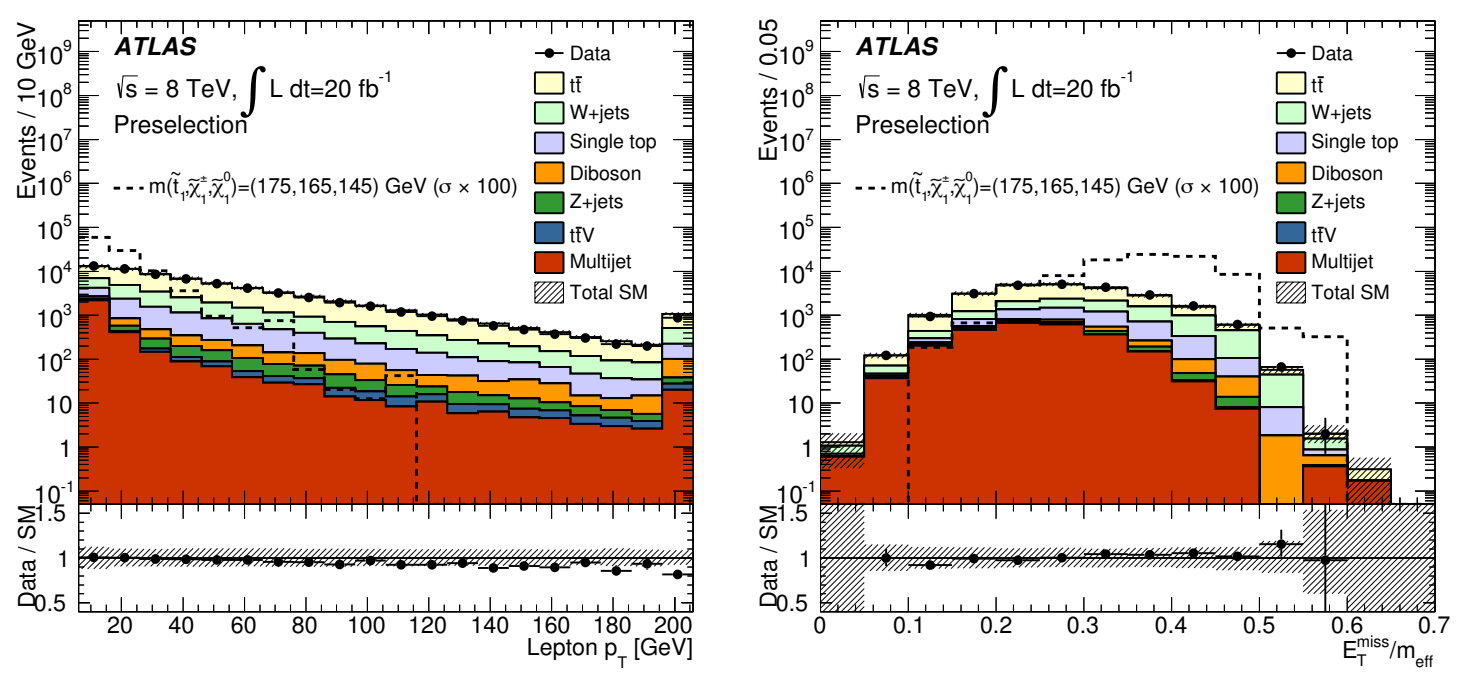

Figure 8. Comparison of data with estimated backgrounds in the lepton $p_{\mathrm{T}}$ (left) and $E_{\mathrm{T}}^{\mathrm{miss}} / m_{\mathrm{eff}}$ (right) distributions. Events are required to satisfy the soft-lepton preselection criteria (cf. table 3), have $m_{\mathrm{T}}>40 \mathrm{GeV}$, and contain two or more jets $\left(p_{\mathrm{T}}>130,25 \mathrm{GeV}\right)$ of which the leading one must not be $b$-tagged while the sub-leading one is required to be $b$-tagged. The $t \bar{t}$ and $W+$ jets backgrounds are normalised using control regions, the multijet background is estimated directly from data, and all other backgrounds are normalised to their theoretical predictions (as described in section 8). The uncertainty band includes statistical and all experimental systematic uncertainties. The last bin includes overflows. A benchmark signal model, with cross-section enhanced by a factor of 100 , is overlaid for comparison.

Two SRs, labelled bCb_med1 and bCb_high, employ event selections based on the presence of one soft lepton and two $b$-tagged jets. They target medium and high stop mass regions, respectively. The complete event selections are listed in table 5 . The bCb_med1 SR employs an $E_{\mathrm{T}}^{\text {miss }}>150 \mathrm{GeV}$ requirement, the lowest possible to retain full $E_{\mathrm{T}}^{\text {miss }}$ trigger efficiency. For models with a heavier $\tilde{t}_{1}$, a higher $E_{\mathrm{T}}^{\text {miss }}$ threshold improves the sensitivity. The dominant background stems from $t \bar{t}$ production and is suppressed by vetoing additional hard jet activity. The variable $H_{\mathrm{T}, 2}$ is defined like $H_{\mathrm{T}}$ but without including the two leading jets. The $\mathrm{bCb}$ high SR omits the jet activity veto to compensate for the loss in signal acceptance associated with the more stringent $E_{\mathrm{T}}^{\mathrm{miss}}$ requirement. Beyond the kinematic $a m_{\mathrm{T} 2}$ bound, the dominant source of background arises from mis-tagged $c$-jets in semileptonic $t \bar{t}$ events, and the production of a $W$ boson in association with heavy-flavour jets. To minimise the mis-tagged background, the $b$-tagging algorithm is operated at the $60 \%$ efficiency working point. A minimum requirement on the invariant mass of the two $b$-tagged jets, $m_{b b}$, is imposed to reduce the contribution from $W+b \bar{b}$ events.

Exclusion results are obtained using a shape-fit in the $a m_{\mathrm{T} 2}$ variable with six bins in the range $[0,500] \mathrm{GeV}$ with a uniform bin width. For all model-independent results, the cut-and-count approach is used but applying an $a m_{\mathrm{T} 2}>170(200) \mathrm{GeV}$ requirement in bCb_med1 (bCb_high). 

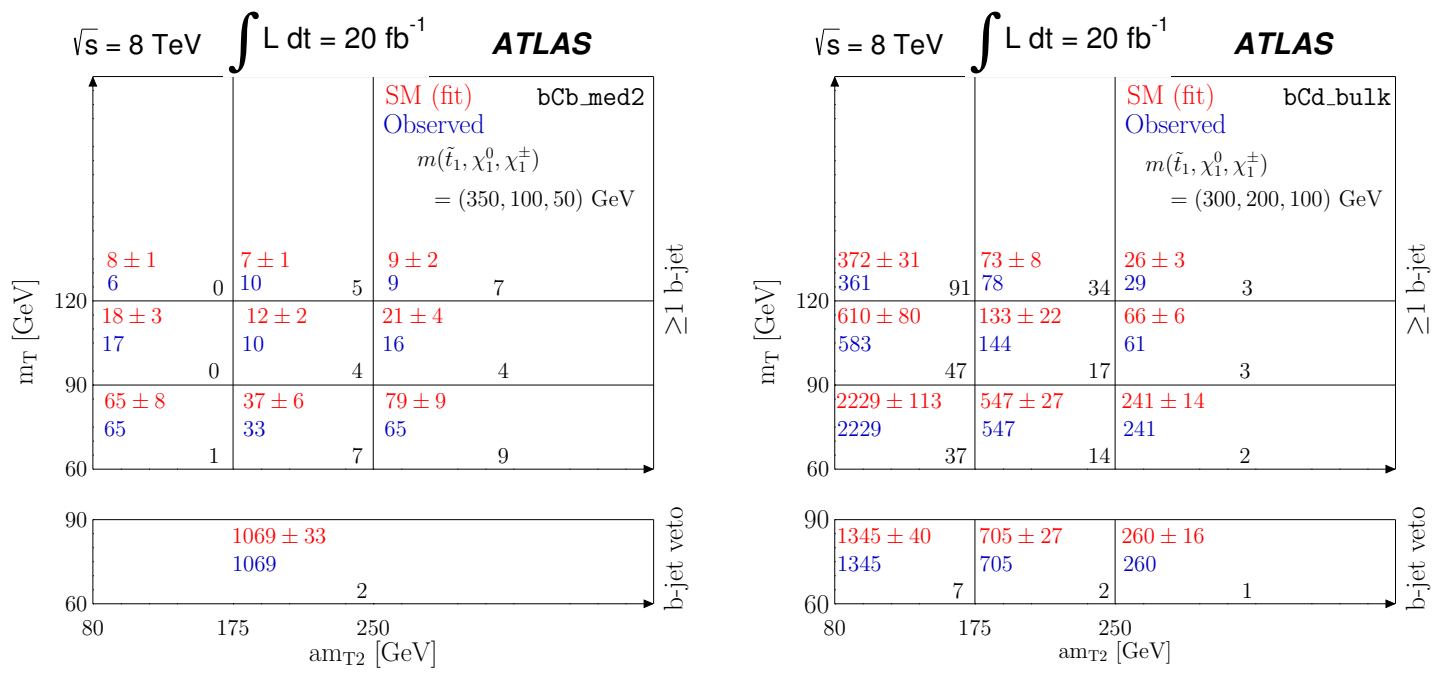

Figure 9. Schematic illustration of the bCb_med2 (left) and bCd_bulk (right) shape-fit binning. More details are given in the caption of figure 6 . The data and estimated background are in perfect agreement in the six bottom bins of the right plot because the fit is configured to use these six bins together with six free parameters; the fit used for the left plot employs the bottom four bins and two free parameters.

A third SR, labelled $\mathrm{bCb}$ med2, also targets intermediate stop masses. It is based on the default lepton selection $\left(p_{\mathrm{T}}>25 \mathrm{GeV}\right)$, and requiring at least two high- $p_{\mathrm{T}} b$-tagged jets to exploit larger $\tilde{t}_{1}-\tilde{\chi}_{1}^{ \pm}$mass splittings than bCb_med1. The full event selection is detailed in the leftmost column in table 6 . The analysis employs a two-dimensional shape-fit technique similar to the one used for tN_diag but in the $m_{\mathrm{T}}$ and $a m_{\mathrm{T} 2}$ variables. Figure 9 (left plot) illustrates the configuration. The highest signal sensitivity is obtained from bins in the top-right region.

Selections for mass hierarchy (c): models with $m_{\tilde{\chi}_{1}^{ \pm}}$just below $m_{\tilde{t}_{1}}$ yield two lowmomentum $b$-jets. The signal selection strategy is based on vetoing events with $b$-tagged jets, assuming both signal $b$-jets are below the jet $p_{\mathrm{T}}$ acceptance of the analysis, and therefore suppressing the $t \bar{t}$ background. The sensitivity for low- $m\left(\tilde{t}_{1}\right)$ models is improved by selecting events with ISR-like jet activity. One SR, labelled bCc_diag, is employed and defined in table 6 . The suffix 'diag' refers to the diagonal region of the $m_{\tilde{\chi}_{1}^{ \pm}}=2 m_{\tilde{\chi}_{1}^{0}}$ scenario, the benchmark region used to optimise this SR. The event selection includes one central lepton $(|\eta|<1.2)$ to suppress the $W+$ jets background, and three or more jets, of which none must satisfy the $b$-tagging criteria. In signal events, two of the three required jets tend to originate from the hadronic $W$ boson decay, while the highest- $p_{\mathrm{T}}$ jet typically arises from ISR. The $b$-veto strongly suppresses $t \bar{t}$ events, leaving $W+$ jets as the dominant background. Requirements on the $\Delta R\left(j_{1}, \ell\right)$ and $E_{\mathrm{T}}^{\text {miss }}$ variables further enhance the signalto-background ratio, by selecting events where the two stops recoil from an ISR jet.

Selections for mass hierarchy (d): signal models with relatively large mass splittings between the three mass states, $\tilde{t}_{1}, \tilde{\chi}_{1}^{ \pm}$, and $\tilde{\chi}_{1}^{0}$, result in events where all particles from the 


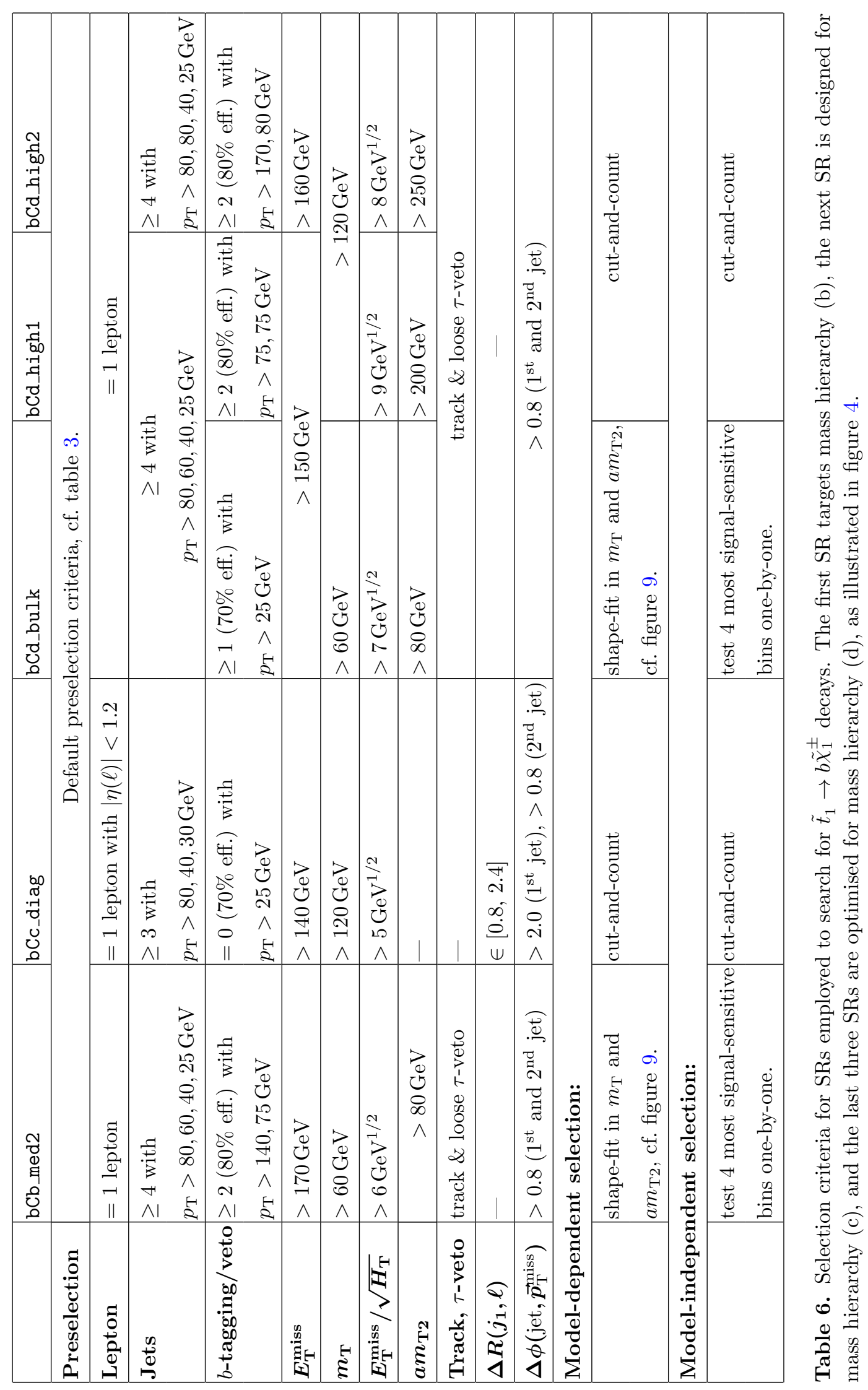


two $\tilde{t}_{1}$ decays are well above the identification $p_{\mathrm{T}}$ thresholds. Three SRs, labelled bCd_bulk, bCd_high1, and bCd_high2, target specific mass regions.

The bCd_bulk SR employs a two-dimensional shape-fit technique with bins in the $m_{\mathrm{T}}-a m_{\mathrm{T} 2}$ plane. Figure 9 (right plot) illustrates the binning. Compared to bCb med2, which uses the same two variables in a shape-fit, a loose and more inclusive event selection is employed. The bCd_high1 and bCd_high2 SRs are based on tight event selections, leading to low expected background yields. Table 6 details the event selections.

\subsection{Selections for the mixed, three- and four-body decays}

Three additional stop decay modes are considered: events where $\tilde{t}_{1} \rightarrow t \tilde{\chi}_{1}^{0}$ and $\tilde{t}_{1} \rightarrow b \tilde{\chi}_{1}^{ \pm}$ decays are both allowed, with the branching ratios of the two decays summing to one; both stops decay via a three-body process $\left(\tilde{t}_{1} \rightarrow b W \tilde{\chi}_{1}^{0}\right)$; and both stops undergo a four-body decay $\left(\tilde{t}_{1} \rightarrow b f f^{\prime} \tilde{\chi}_{1}^{0}\right)$.

In the mixed decay mode, models with a very large and a very small $\mathcal{B R}\left(\tilde{t}_{1} \rightarrow t \tilde{\chi}_{1}^{0}\right)$ are well covered by the SRs targeting the pure $\tilde{t}_{1} \rightarrow t \tilde{\chi}_{1}^{0}$ and $\tilde{t}_{1} \rightarrow b \tilde{\chi}_{1}^{ \pm}$decays, respectively. A dedicated SR with the label tNbC_mix is optimised for models with $\mathcal{B R}\left(\tilde{t}_{1} \rightarrow t \tilde{\chi}_{1}^{0}\right) \sim 0.5$. It employs a requirement on the topness variable, which was designed specifically for the mixed decay mode, to suppress the dominant dileptonic $t \bar{t}$ background. Diboson events that pass the selection tend to have a leptonic $W$ boson decay and a hadronic $W$ or $Z$ decay, accompanied by at least two additional jets. Large $E_{\mathrm{T}}^{\mathrm{miss}}$ can be generated by the neutrino when the diboson system is sufficiently boosted; the two additional jets hence typically arise from ISR activity. The diboson background is suppressed by placing a loose upper requirement on the three-jet invariant mass, $m_{j j j}$. The jet-jet pair with an invariant mass above $60 \mathrm{GeV}$ that has the smallest $\Delta R$ is selected to form the hadronic $V$ boson. The mass $m_{j j j}$ is reconstructed from the third jet closest in $\Delta R$ to the hadronic $V$ boson momentum vector. Table 7 lists the entire event selection.

A dedicated SR labelled 3body is optimised for the three-body decay mode. Compared to the scenario with on-shell top quarks, three-body decays yield the same final state objects but with significantly lower momenta, although typically still above the reconstruction thresholds. The dileptonic $t \bar{t}$ background is separated from signal in the very low $a m_{\mathrm{T} 2}$ regime. The three-body signal peaks in $a m_{\mathrm{T} 2}$ below around $100 \mathrm{GeV}$ due to the kinematic construction of the variable and the fact that $m\left(\tilde{t}_{1}\right)-m\left(\tilde{\chi}_{1}^{0}\right)$ is below the top quark mass. A two-dimensional shape-fit technique using the $m_{\mathrm{T}}$ and $a m_{\mathrm{T} 2}$ variables is employed, similar to that used in bCd_bulk and bCb_med2, but with different binning. The configuration is illustrated in figure 6 (right plot). Fine binning is used in the low $a m_{\mathrm{T} 2}$ region where the highest signal sensitivity is obtained. The full 3body event selection is detailed in table 7 .

The four-body decay mode is characterised by events with final state objects that tend to have even lower momenta than for three-body decays. The selections based on a soft lepton designed for the overall 'compressed' mass hierarchy (a) provide good search sensitivity for this scenario. 


\begin{tabular}{|c|c|c|}
\hline & tNbC_mix & 3body \\
\hline Preselection & \multicolumn{2}{|c|}{ Default preselection criteria, cf. table 3 . } \\
\hline Lepton & \multicolumn{2}{|c|}{$=1$ lepton } \\
\hline Jets & $\geq 4$ jets with $p_{\mathrm{T}}>80,70,50,25 \mathrm{GeV}$ & $\geq 4$ jets with $p_{\mathrm{T}}>80,25,25,25 \mathrm{GeV}$ \\
\hline$b$-tagging & $\geq 1 b$-tag $(70 \%$ eff. $)$ with $p_{\mathrm{T}}>60 \mathrm{GeV}$ & $\geq 1 b$-tag $(70 \%$ eff. $)$ with $p_{\mathrm{T}}>25 \mathrm{GeV}$ \\
\hline$E_{\mathrm{T}}^{\text {miss }}$ & $>270 \mathrm{GeV}$ & $>150 \mathrm{GeV}$ \\
\hline$m_{\mathrm{T}}$ & $>130 \mathrm{GeV}$ & $>60 \mathrm{GeV}$ \\
\hline$a m_{\mathrm{T} 2}$ & $>190 \mathrm{GeV}$ & $>80 \mathrm{GeV}$ \\
\hline topness & $>2$ & - \\
\hline$m_{j j j}$ & $<360 \mathrm{GeV}$ & - \\
\hline $\boldsymbol{E}_{\mathrm{T}}^{\mathrm{miss}} / \sqrt{\boldsymbol{H}_{\mathrm{T}}}$ & $>9 \mathrm{GeV}^{1 / 2}$ & $>5 \mathrm{GeV}^{1 / 2}$ \\
\hline$\tau$-veto & \multicolumn{2}{|c|}{ loose } \\
\hline$\Delta \phi\left(\right.$ jet $\left._{i}, \vec{p}_{\mathrm{T}}^{\mathrm{miss}}\right)$ & $>0.6(i=1,2)$ & $>0.2(i=1,2)$ \\
\hline$\Delta \phi\left(\ell, \vec{p}_{\mathrm{T}}^{\text {miss }}\right)$ & $>0.6$ & $>1.2$ \\
\hline$\Delta R\left(\ell\right.$, jet $\left._{i}\right)$ & $<2.75(i=1)$ & $>1.2(i=1),>2.0(i=2)$ \\
\hline$\Delta R(\ell, b$-jet $)$ & $<3.0$ & - \\
\hline \multicolumn{3}{|c|}{ Model-dependent selection: } \\
\hline & cut-and-count & shape-fit in $m_{\mathrm{T}}$ and $a m_{\mathrm{T} 2}$, cf. figure 6 . \\
\hline \multicolumn{3}{|c|}{ Model-independent selection: } \\
\hline & cut-and-count & $\begin{array}{l}\text { test } 4 \text { most signal-sensitive } \\
\text { bins one-by-one. }\end{array}$ \\
\hline
\end{tabular}

Table 7. Selection criteria for the two SRs employed to search for the mixed $\tilde{t}_{1} \rightarrow t \tilde{\chi}_{1}^{0}$ and $\tilde{t}_{1} \rightarrow b \tilde{\chi}_{1}^{ \pm}$ decay mode (left), and the three-body decay, $\tilde{t}_{1} \rightarrow b W \tilde{\chi}_{1}^{0}$ (right).

\section{Background estimates}

The dominant sources of background are the production of $t \bar{t}$ events and $W+$ jets where the $W$ decays leptonically. Other background processes considered are single top, dibosons, $Z+$ jets, $t \bar{t}$ produced with a vector boson $(t \bar{t} V)$, and multijet events.

The $t \bar{t}$ and $W+$ jets backgrounds are estimated by isolating each of them in a dedicated control region, normalising simulation to match data in that control region, and then using simulation to extrapolate the background predictions into the SR. A detailed description of the method and its validation are given below.

The multijet background is estimated from data using a matrix method described in refs. [130, 131]. The contribution is found to be negligible for all but the soft-lepton selec- 
tions. All other (small) backgrounds are determined entirely from simulation, normalised to the most accurate theoretical cross-sections available (cf. section 5).

\subsection{Control regions}

Each cut-and-count analysis has two orthogonal CRs which are enriched in either $t \bar{t}$ events (TCR) or $W+$ jets events (WCR). These CRs are used to normalise the corresponding backgrounds in data, specifically for the associated SR. For the two-dimensional shape-fits, bins enriched in $t \bar{t}$ events are already part of the SR and act as a TCR, while additional bins are used to normalise the $W+$ jets background. The one-dimensional shape-fits are set up similar to the cut-and-count analyses, with two additional bins acting as TCR and WCR. The CRs are designed to select events as similar as possible to those selected by the corresponding SR while keeping the contamination from other backgrounds and potential signal low. The CRs are also chosen to retain a sufficiently large number of events to not be limited by the statistical precision. This background estimation approach improves the robustness against potential mis-modelling effects in simulation since the dependence on simulation is reduced, and hence it reduces the uncertainties on the background estimates.

A likelihood fit is performed for each analysis, involving all associated bins: SR, TCR, WCR for cut-and-count or all bins of a shape-fit. Each bin is modelled by a separate probability density function based on a Poisson term, where the expected number of events is given by the sum over all background processes, and optionally a signal model. The normalisation of the $t \bar{t}$ and $W+$ jets backgrounds is controlled by two free parameters in the fit. ${ }^{10}$ To obtain a set of background predictions that is independent of the observation in the SRs, the fit can be configured to use only the CRs to constrain the fit parameters: the SR bins (or signal-sensitive bins in shape-fits) are removed from the likelihood and any potential signal contribution is neglected everywhere. This fit configuration, referred to as the backgroundonly fit, is used throughout this section. The treatment of systematic uncertainties in the likelihood fits is discussed in section 9. To quantify a potential excess, or to derive exclusion limits, the SR bins are included in the likelihood, as further detailed in section 10.

The key observable used to define the TCR and WCR for most analyses is the transverse mass. Figure 10 shows the $m_{\mathrm{T}}$ distribution for events passing the preselection defined in section 7.1 and with $\geq 1$ (left) or $\geq 2 b$-tagged jets (right) using the $70 \%$ and $80 \%$ working points, respectively. The $t \bar{t}$ and $W+$ jets backgrounds drop sharply beyond the $W$ boson mass, while signal events can exceed this kinematic endpoint due to the two additional LSPs in the event. In all cut-and-count analyses the CRs differ from the associated SR by the $m_{\mathrm{T}}$ requirement, which is set to $60 \mathrm{GeV}<m_{\mathrm{T}}<90 \mathrm{GeV}$ for the CRs. For the four SRs based on a soft lepton, which all employ one-dimensional shape-fits, the two CRs are defined by loosening the $E_{\mathrm{T}}^{\text {miss }}$ and $m_{\mathrm{T}}$ requirements (bCa_med and bCa_low), or the softlepton selection of $6(7) \mathrm{GeV}<p_{\mathrm{T}}(\ell)<25 \mathrm{GeV}$ for muons (electrons) in the SR is changed to a $p_{\mathrm{T}}(\ell)>25 \mathrm{GeV}$ requirement in the $\mathrm{CRs}$ (bCb_med1 and $\mathrm{bCb} \_$high). All WCRs have a $b$-tag veto instead of a $b$-tag requirement to reduce the $t \bar{t}$ contamination; consequently

\footnotetext{
${ }^{10}$ For some of the two-dimensional shape-fits, the $t \bar{t}$ and $W+$ jets backgrounds are controlled by more than two parameters, as discussed later in the text.
} 

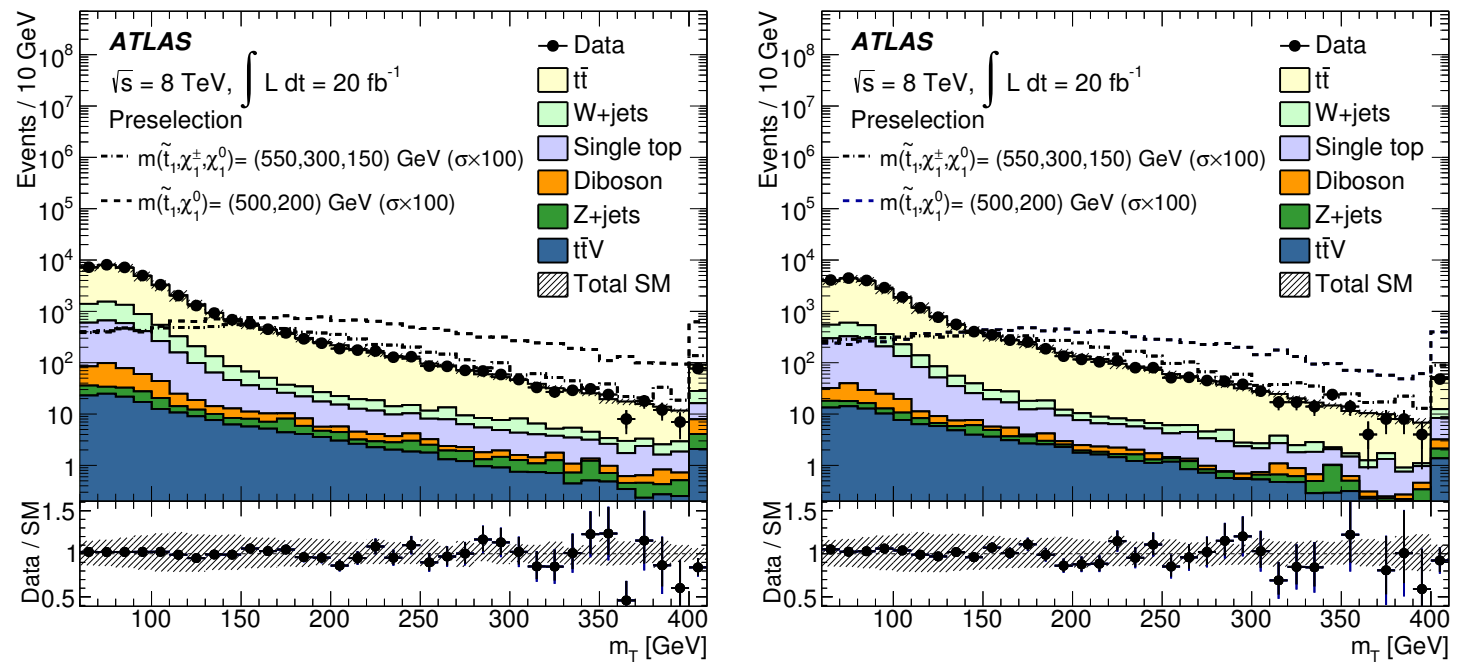

Figure 10. Distribution of the transverse mass, $m_{\mathrm{T}}$, for events that pass the same preselection as used in figure 5 and with at least one (left) or at least two (right) b-tagged jets. The uncertainty band includes statistical and all experimental systematic uncertainties. The last bins include the overflows. Benchmark signal models with cross-sections enhanced by a factor of 100 are overlaid for comparison.

all requirements related to the presence of a $b$-jet are removed. The $b$-tag requirement used in all but one SR enhances the heavy-flavour contribution of the $W+$ jets background, while the WCRs with the $b$-tag veto predominantly select light-flavour $W+$ jets events. A systematic uncertainty and a dedicated validation related to this effect are described in section 9 and in the next subsection, respectively. The TCRs employ the same $b$-tagging requirement as used in the associated SRs, except for bCc_diag where the $b$-tag veto in the $\mathrm{SR}$ is turned into $\mathrm{a} \geq 1 b$-tag requirement in the TCR (using the same $b$-tagging working point as for the $b$-tag veto in the SR). Furthermore, some other kinematic requirements are slightly loosened or removed to increase the event yields in the CRs. The event selections for all CRs associated with the cut-and-count or the one-dimensional shape-fit analyses are specified in table 8. In each analysis the same two CRs are used for the model-dependent and the model-independent fit configurations.

The four two-dimensional shape-fits ( $t N_{\text {_diag, }}$ 3body, bCb_med2, and bCd_bulk) have built-in bins enhanced in $t \bar{t}$ events, which act as TCRs, while additional WCR bins are added with a $b$-tag veto and with a $60 \mathrm{GeV}<m_{\mathrm{T}}<90 \mathrm{GeV}$ requirement (as can be seen in figures 6 and 9). For the background-only fits, the WCRs and the subset of the TCR shape-fit bins with $m_{\mathrm{T}}<90 \mathrm{GeV}$ are used to constrain the likelihood fit. The $t \bar{t}$ and $W+$ jets backgrounds are normalised separately in each $E_{\mathrm{T}}^{\text {miss }}$ or $a m_{\mathrm{T} 2}$ slice in the $\mathrm{tN}_{\mathrm{N}}$ diag and bCd_bulk shape-fits, as these two have sufficiently large numbers of events in the low$m_{\mathrm{T}}$ bins. Thus, there are three $t \bar{t}$ and three $W+$ jets normalisation parameters, which are applied to all $m_{\mathrm{T}}$ bins in the given $E_{\mathrm{T}}^{\text {miss }}$ or $a m_{\mathrm{T} 2}$ range. This approach increases the robustness of the fit against potential mis-modelling in the simulation at the expense of a reduced statistical precision. For the other two shape-fits with lower event yields, 


\begin{tabular}{|c|c|c|c|c|c|c|}
\hline \multirow[t]{2}{*}{ Analysis } & \multirow[t]{2}{*}{ Variable } & \multicolumn{2}{|c|}{ Control regions } & \multicolumn{2}{|c|}{ Validation regions } & \multirow{2}{*}{$\begin{array}{c}\text { Signal reg. } \\
\text { SR }\end{array}$} \\
\hline & & TCR & WCR & TVR & WVR & \\
\hline All tN_* & $\begin{array}{l}m_{\mathrm{T}} \\
N_{b}\end{array}$ & $\begin{array}{l}{[60,90]} \\
\geq 1\end{array}$ & $\begin{array}{l}{[60,90]} \\
=0\end{array}$ & $\begin{array}{l}{[90,120]} \\
\geq 1\end{array}$ & $\begin{array}{l}{[90,120]} \\
=0\end{array}$ & $\begin{array}{l}>[140,200] \\
\geq 1\end{array}$ \\
\hline $\mathrm{tN}$ med & $a m_{\mathrm{T} 2}$ & $>120$ & $>120$ & $>120$ & $>120$ & $>170$ \\
\hline tN_high & $\begin{array}{l}a m_{\mathrm{T} 2} \\
m_{\mathrm{T} 2}^{\tau} \\
E_{\mathrm{T}}^{\mathrm{miss}} \\
H_{\mathrm{T}}^{\mathrm{sig}}\end{array}$ & $\begin{array}{l}- \\
- \\
>225 \\
>8.8\end{array}$ & $\begin{array}{l}- \\
- \\
>225 \\
>8.8\end{array}$ & $\begin{array}{l}- \\
- \\
>225 \\
>8.8\end{array}$ & $\begin{array}{l}- \\
- \\
>225 \\
>8.8\end{array}$ & $\begin{array}{l}>170 \\
>120 \\
>320 \\
>12.5\end{array}$ \\
\hline tN_boost & $\begin{array}{l}a m_{\mathrm{T} 2} \\
E_{\mathrm{T}}^{\text {miss }} \\
\text { topness }\end{array}$ & $\begin{array}{l}>130 \\
>260 \\
-\end{array}$ & $\begin{array}{l}>130 \\
>260 \\
-\end{array}$ & $\begin{array}{l}>130 \\
>260 \\
-\end{array}$ & $\begin{array}{l}>130 \\
>260 \\
-\end{array}$ & $\begin{array}{l}>145 \\
>315 \\
>7\end{array}$ \\
\hline All bCa_* & $\begin{array}{l}p_{\mathrm{T}}(\ell) \\
N_{b}\end{array}$ & $\begin{array}{l}>6(7) \\
\geq 1\end{array}$ & $\begin{array}{l}>6(7) \\
=0\end{array}$ & $\begin{array}{l}{[6(7), 25]} \\
\geq 1\end{array}$ & $\begin{array}{l}{[6(7), 25]} \\
\geq 1\end{array}$ & $\begin{array}{l}{[6(7), 25 / 50]} \\
\geq 1\end{array}$ \\
\hline bCa_low & $\begin{array}{l}E_{\mathrm{T}}^{\text {miss }} \\
m_{\mathrm{T}} \\
E_{\mathrm{T}}^{\text {miss }} / m_{\text {eff }}\end{array}$ & $\begin{array}{l}{[200,250]} \\
{[90,120]} \\
-\end{array}$ & $\begin{array}{l}{[200,250]} \\
{[90,120]} \\
-\end{array}$ & $\begin{array}{l}{[250,370]} \\
>90 \\
-\end{array}$ & $\begin{array}{l}{[250,370]} \\
>90 \\
>0.35\end{array}$ & $\begin{array}{l}>370 \\
>90 \\
>0.35\end{array}$ \\
\hline bCa_med & $\begin{array}{l}E_{\mathrm{T}}^{\mathrm{miss}} \\
m_{\mathrm{T}} \\
E_{\mathrm{T}}^{\mathrm{miss}} / m_{\mathrm{eff}}\end{array}$ & $\begin{array}{l}{[200,250]} \\
{[100,120]} \\
-\end{array}$ & $\begin{array}{l}{[200,250]} \\
{[100,120]} \\
-\end{array}$ & $\begin{array}{l}{[250,300]} \\
>100 \\
-\end{array}$ & $\begin{array}{l}{[250,300]} \\
>100 \\
>0.3\end{array}$ & $\begin{array}{l}>300 \\
>100 \\
>0.3\end{array}$ \\
\hline All bCb_* & $\begin{array}{l}p_{\mathrm{T}}(\ell) \\
N_{b} \\
m_{\mathrm{T}} \\
m_{b b}\end{array}$ & $\begin{array}{l}>25 \\
=2 \\
- \\
-\end{array}$ & $\begin{array}{l}>25 \\
=0 \\
{[40,80]} \\
-\end{array}$ & $\begin{array}{l}{[6(7), 25]} \\
=2 \\
- \\
<150\end{array}$ & & $\begin{array}{l}{[6(7), 25]} \\
=2 \\
- \\
>150\end{array}$ \\
\hline $\mathrm{bCb}$ med1 & $a m_{\mathrm{T} 2}$ & - & - & - & & $>170$ \\
\hline bCb_high & $\begin{array}{l}a m_{\mathrm{T} 2} \\
E_{\mathrm{T}}^{\text {miss }}\end{array}$ & $\begin{array}{l}- \\
>250\end{array}$ & $\begin{array}{l}- \\
>150\end{array}$ & $\begin{array}{l}- \\
>250\end{array}$ & & $\begin{array}{l}>200 \\
>250\end{array}$ \\
\hline All bCc_*, bCd_* & $m_{\mathrm{T}}$ & {$[60,90]$} & {$[60,90]$} & {$[90,120]$} & {$[90,120]$} & $>120$ \\
\hline bCc_diag & $N_{b}$ & $\geq 1$ & $=0$ & $\geq 1$ & $=0$ & $=0$ \\
\hline All bCd_* & $N_{b}$ & $\geq 2$ & $=0$ & $\geq 2$ & $=0$ & $\geq 2$ \\
\hline bCd_high1 & $a m_{\mathrm{T} 2}$ & $>120$ & $>200$ & $>120$ & $>200$ & $>200$ \\
\hline bCd_high2 & $a m_{\mathrm{T} 2}$ & $>120$ & $>250$ & $>120$ & $>250$ & $>250$ \\
\hline tNbC_mix & $\begin{array}{l}m_{\mathrm{T}} \\
N_{b} \\
a m_{\mathrm{T} 2} \\
E_{\mathrm{T}}^{\text {miss }} \\
E_{\mathrm{T}}^{\text {miss }} / \sqrt{H_{\mathrm{T}}} \\
\end{array}$ & $\begin{array}{l}{[60,90]} \\
\geq 1 \\
>120 \\
>170 \\
>5\end{array}$ & $\begin{array}{l}{[60,90]} \\
=0 \\
>120 \\
>170 \\
>5\end{array}$ & $\begin{array}{l}{[90,120]} \\
\geq 1 \\
>120 \\
>170 \\
>5\end{array}$ & $\begin{array}{l}{[90,120]} \\
=0 \\
>120 \\
>170 \\
>5\end{array}$ & $\begin{array}{l}>130 \\
\geq 1 \\
>190 \\
>270 \\
>9\end{array}$ \\
\hline
\end{tabular}

Table 8. Event selections for control regions, validation regions, and the signal regions of the model-independent selection (defined in tables 4-6) associated with cut-and-count or onedimensional shape-fit analyses. The asterisk symbol ' $*$ ' is used as a wildcard to describe variable requirements common to several regions. Only one validation region is defined for the $\mathrm{bCb}$ med1 and $\mathrm{bCb}$ high selections. Variables for which the requirements are the same between the regions are not listed. Requirements related to the presence of a $b$-tagged jet are removed in all selections with a $b$-tag veto (WCRs and WVRs). All units are in GeV except for unitless quantities and $E_{\mathrm{T}}^{\text {miss }} / \sqrt{H_{\mathrm{T}}}$ which is quoted in $\mathrm{GeV}^{1 / 2}$. 
3body and $\mathrm{bCb} \_$med2, one $t \bar{t}$ and one $W+$ jets normalisation parameter is applied across all bins. All shape-fit bins are used to extract model-dependent exclusion limits, while a subset is used for the model-independent results and for the background only fits. This subset includes all bins with $m_{\mathrm{T}}<90 \mathrm{GeV}$, acting as $\mathrm{CR}$ bins, and in addition for the model-independent results one signal-sensitive bin is included.

Top pair and $W+$ jets production accounts for $70-80 \%$ of events in the TCRs and WCRs. The signal contamination, for all signal models studied and all CRs, is typically at the percent level and never exceeds $10 \%$. It is explicitly taken into account when setting model-dependent exclusion limits.

Table 9 shows the background predictions in each SR. The number of $t \bar{t}$ and $W+$ jets events are estimated using the background-only fit configuration. For the four twodimensional shape-fits, the background predictions are given for the four bins with the highest signal-sensitivity. The quoted uncertainties include all statistical and systematic effects, described in section 9. The numbers of $t \bar{t}$ events normalised in the various TCRs are compatible with the predictions entirely based on simulation and the theoretical crosssection, while the $W+$ jets estimates are about $30 \%$ lower than, but nonetheless compatible with the predictions from simulation normalised to the theoretical cross-section. Tables showing the estimated and fitted number of background events in the CRs, validation regions, and SRs of all analyses are shown in appendix B.

\subsection{Validation}

The background fit predictions are validated using dedicated event samples. For each cut-and-count and one-dimensional shape-fit analysis one or more dedicated validation regions (VRs) are defined for the $t \bar{t}$ and $W+$ jets backgrounds. The VRs are designed to be kinematically close to the associated SRs to test the background estimates in regions of phase space as similar as possible to the SRs. For most analyses the associated VRs are defined following a similar strategy as used for the CRs but with a $90 \mathrm{GeV}<m_{\mathrm{T}}<120 \mathrm{GeV}$ requirement, which leads to a set of events orthogonal to both the associated CRs and the SR. The event selections for the $t \bar{t}$ and $W+$ jets VRs, TVR and WVR respectively, are given in table 8 . Another set of $t \bar{t}$ validation regions, referred to as TVR2 but not shown in the table, is defined where applicable by inverting the SR requirement on $a m_{\mathrm{T} 2}$ while keeping all other requirements the same as in the SR. For the four two-dimensional shapefits, a subset of the shape-fit bins in the region falling in between that dominated by $t \bar{t}$ and the region enhanced by a potential signal is used for the $t \bar{t}$ background validation. These signal-depleted shape-fit bins are referred to as validation bins.

For the cut-and-count and one-dimensional shape-fit analyses, the VRs are not used in any fit configuration to constrain the fit parameters. The validation bins of the twodimensional shape-fits, on the other hand, are not used in the background-only fit configuration but are included in the model-dependent fit configuration. The number of background events in each VR or validation bin is predicted by the background-only fit (using simulation for the extrapolation) and compared to the data, as shown in the upper panel of figure 11. The lower panel shows the pull for each bin, where the pull is defined as the difference between the predicted background and the observed number of events divided 


\begin{tabular}{|c|c|c|c|c|c|c|c|}
\hline & $\begin{array}{lll}1 & 1\end{array}$ & 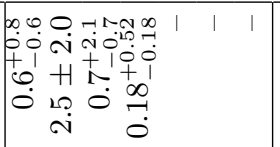 & 1 & 111 & $\begin{array}{llll}1 & 1 & 1 & 1\end{array}$ & $\begin{array}{llll}1 & 1 & 1 & 1\end{array}$ & 111 \\
\hline & 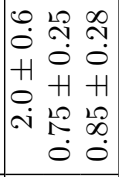 & 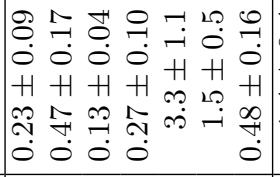 & & 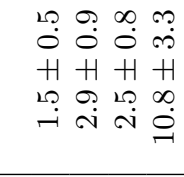 & 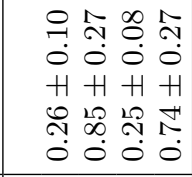 & 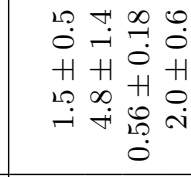 & 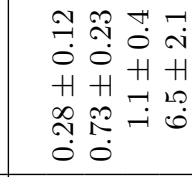 \\
\hline & 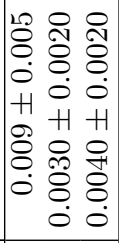 & 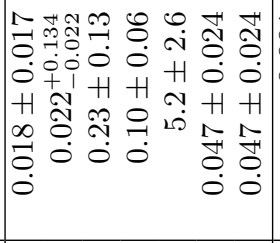 & & 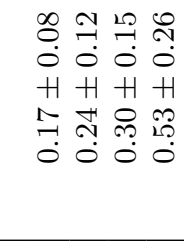 & 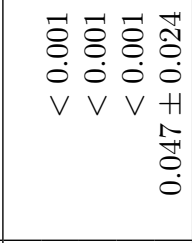 & 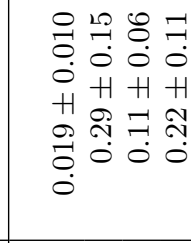 & 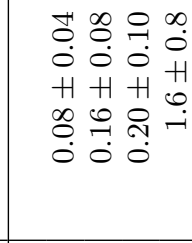 \\
\hline & 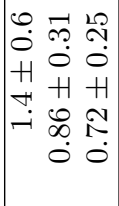 & 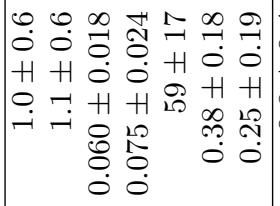 & & 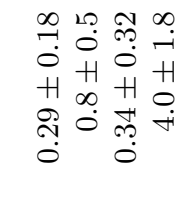 & 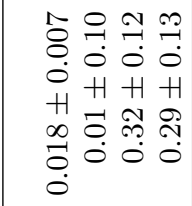 & 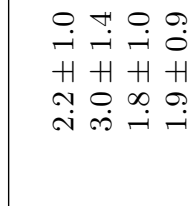 & 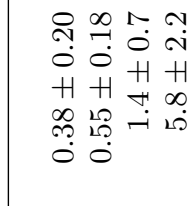 \\
\hline & 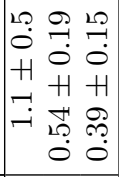 & 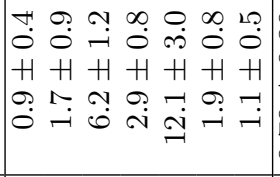 & & 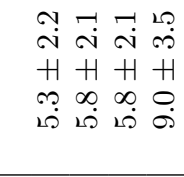 & 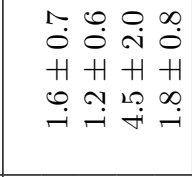 & 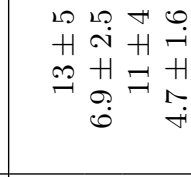 & 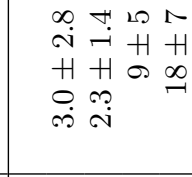 \\
\hline & 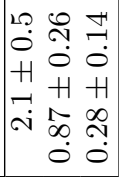 & 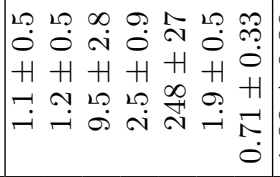 & & 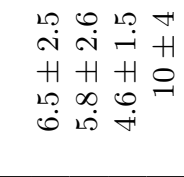 & 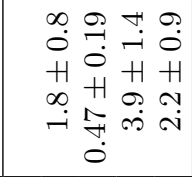 & 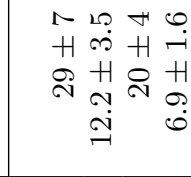 & 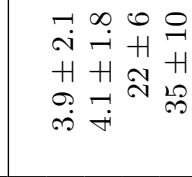 \\
\hline & 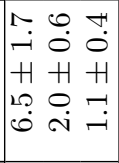 & 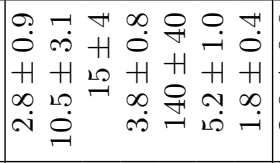 & & 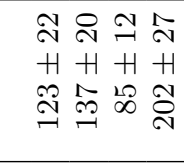 & 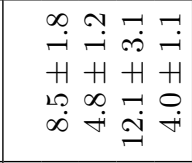 & 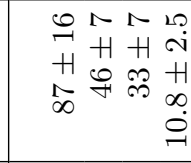 & 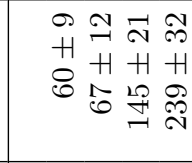 \\
\hline & 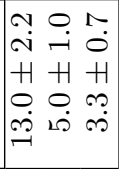 & 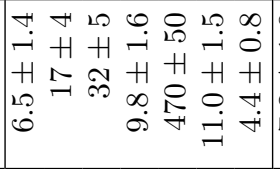 & & 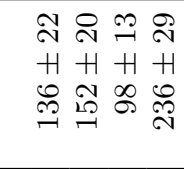 & 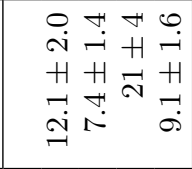 & 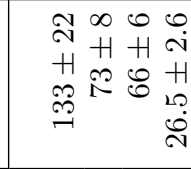 & 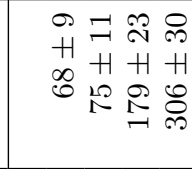 \\
\hline & 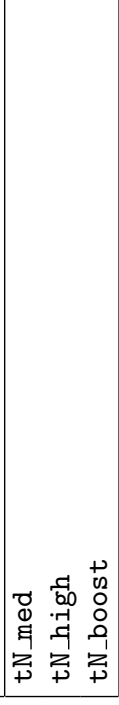 & 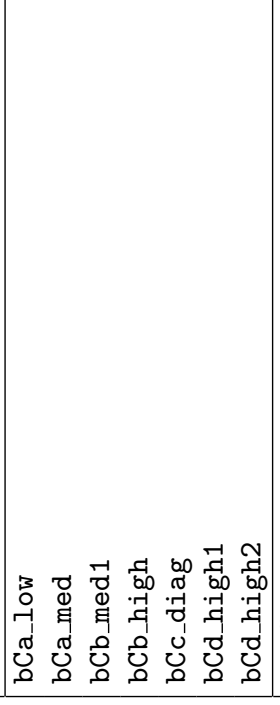 & & 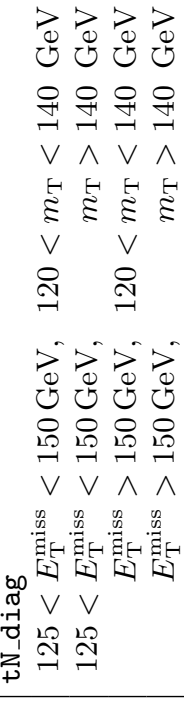 & 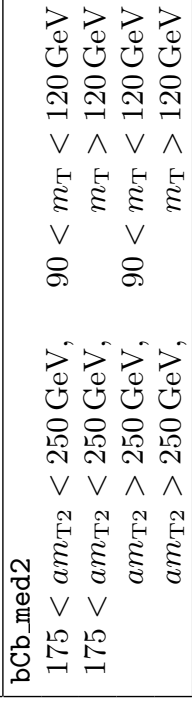 & 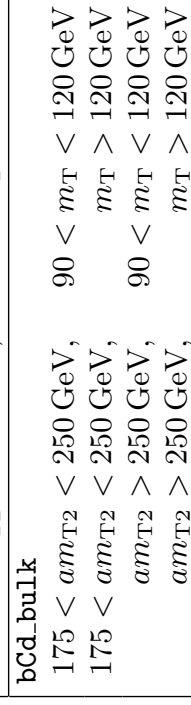 & 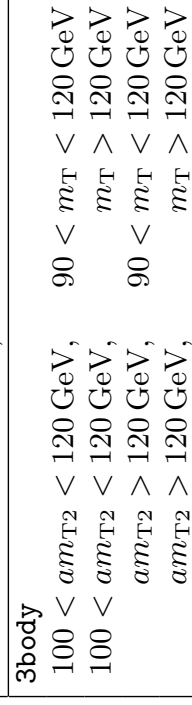 \\
\hline
\end{tabular}

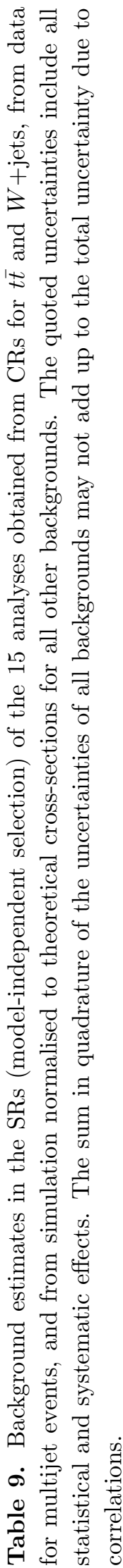


by the total uncertainty. The latter is given by the full uncertainty of the prediction (described in section 9) added in quadrature with the statistical uncertainty of the observed number of events. No indication of background mis-modelling is found. VRs or validation bins belonging to different analyses can share events, and the systematic uncertainties are correlated across different regions and bins.

Several other cross checks are performed to further validate the background estimations. For the SRs requiring more than two jets, dileptonic $t \bar{t}$ events can pass the event selection only if they contain additional jets beyond the two $b$-jets from the leading-order description of the decay. The modelling of these additional jets, which in the simulation arise from radiation or higher-order-corrections, and which is relevant for the background estimation, is validated using a dedicated sample. The event selection is based on requiring one isolated electron and one oppositely-charged, isolated muon, as well as two or more jets of which at least one is $b$-tagged using the $70 \%$ working point. This selects a clean sample of $t \bar{t}$ events, which is used in figure 12 (left) to compare the jet multiplicity distributions between data and simulation. Data is modelled sufficiently well within the systematic uncertainties. Further dedicated validation samples are used to test the modelling of the $t \bar{t}$ background with a $\tau_{\text {had }}$ or isolated track. These samples are based on the common event preselection and inverting either the track- or $\tau$-veto. The simulation is found to model data well within uncertainties.

The $W+$ jets light- vs heavy-flavour composition in the WCR can be different from that in the SR. A dedicated validation is performed by selecting a sample enriched with $W+$ heavy-flavour jets events. The event selection is based on exactly one isolated lepton, and exactly three jets (the fourth jet veto reduces $t \bar{t}$ events), of which at least one is $b$-tagged. Furthermore, events are required to have $60 \mathrm{GeV}<m_{\mathrm{T}}<90 \mathrm{GeV}, E_{\mathrm{T}}^{\text {miss }}>$ $150 \mathrm{GeV}$, and the two jets with the highest $b$-tagging weights are required to yield an invariant mass below $80 \mathrm{GeV}$ and to have a limited separation in $\eta-\phi$ space to increase the sensitivity to pair-produced heavy-flavour jets in association with a $W$ boson. The selected sample of 166 events has a predicted $W$ +heavy-flavour jets component of about $40 \%$; data are found to be in good agreement with simulation, predicting 171 events, when the overall $W+$ jets background is normalised to match data in a $b$-veto control region. ${ }^{11}$

Another dedicated validation sample is constructed to test the background prediction for $t \bar{t}$ produced with a $Z$ boson that decays to two neutrinos, $t \bar{t} Z(\rightarrow \nu \bar{\nu})$. This process represents an irreducible background that becomes important for SRs with stringent requirements on kinematic variables, such as $t N \_$high or $t N \_b o o s t$. The validation strategy is to select $t \bar{t}$ events produced in association with a photon, $t \bar{t} \gamma$. This process closely resembles $t \bar{t} Z(\rightarrow \nu \bar{\nu})$ in terms of Feynman diagrams and kinematic properties when the vector boson $p_{\mathrm{T}}$ is well above $m_{Z}$. The event selection is based on one isolated lepton, four or more jets with at least one $b$-tag, one high- $p_{\mathrm{T}}$ photon, as well as requirements on modified versions of $m_{\mathrm{T}}$ and $E_{\mathrm{T}}^{\text {miss }}$ where photons are treated as invisible particles. Figure 12 (right) compares data and background predictions, illustrating the accuracy of data modelling.

\footnotetext{
${ }^{11}$ The $W+$ jets background is normalised using the WCR associated with bCc_diag, which requires three or more jets with a jet $p_{\mathrm{T}}$ selection similar to that used in the validation sample.
} 


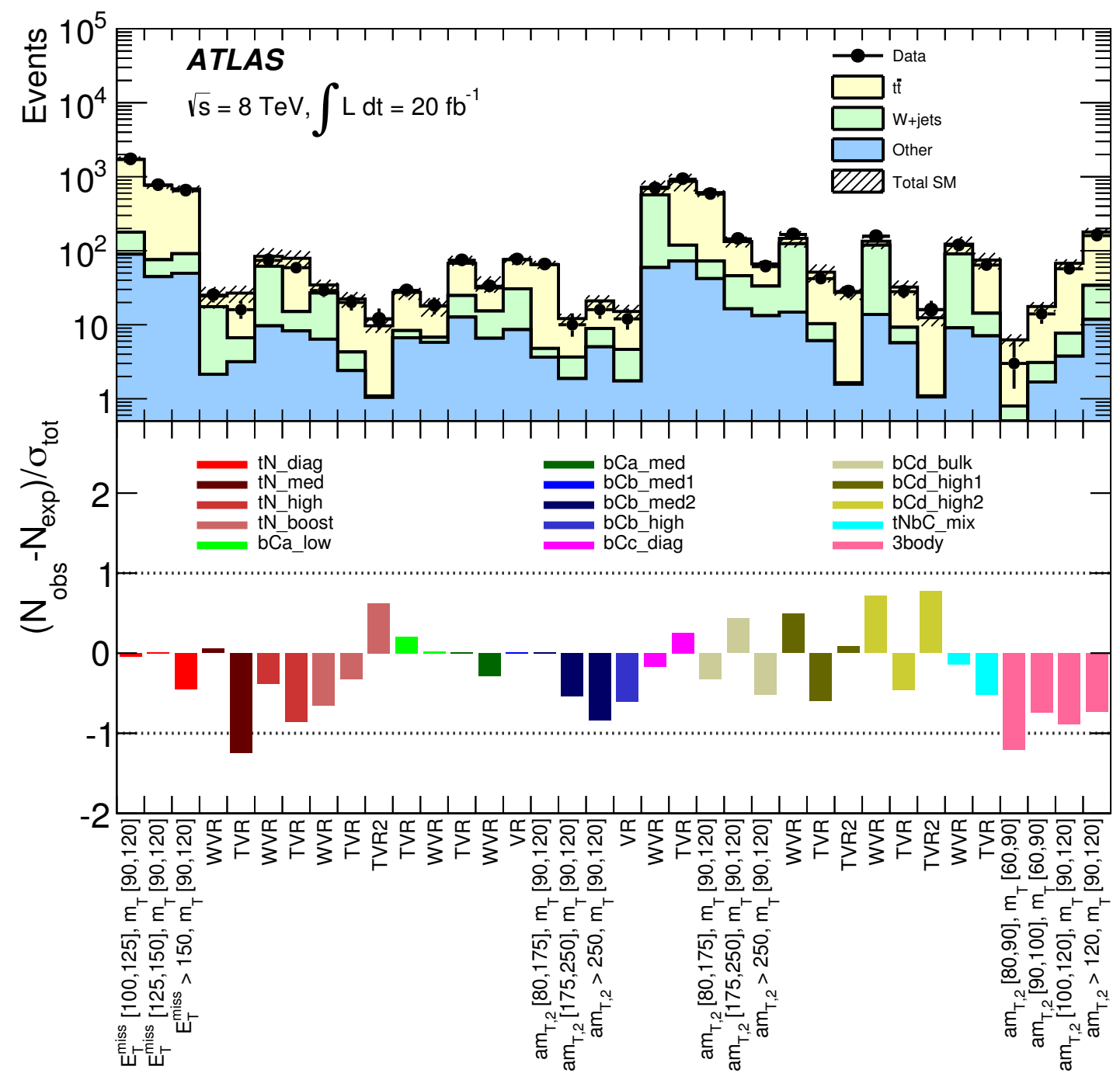

Figure 11. The upper panel compares data with background predictions in the VRs of the cut-and-count and one-dimensional shape-fit analyses as well as the validation bins of the twodimensional shape-fit analyses. The lower panel shows the pull of the same bins. The $t \bar{t}$ and $W+$ jets background estimates are obtained using the background-only fit to the CRs (described in the text). All statistical and systematic uncertainties are included.

The sample of 104 events has a purity in $t \bar{t} \gamma$ of more than $70 \%$. The production of $t \bar{t} \gamma$ events is estimated using simulation, based on the same generator (MADGRAPH) as used for the $t \bar{t} Z$ process, and normalised to the NLO theoretical cross-section [132].

\section{Systematic uncertainties}

The systematic uncertainties affecting the results can be divided into two classes: uncertainties due to theoretical predictions and modelling, and uncertainties stemming from experimental effects. The impact of both types of uncertainty is evaluated for all back- 

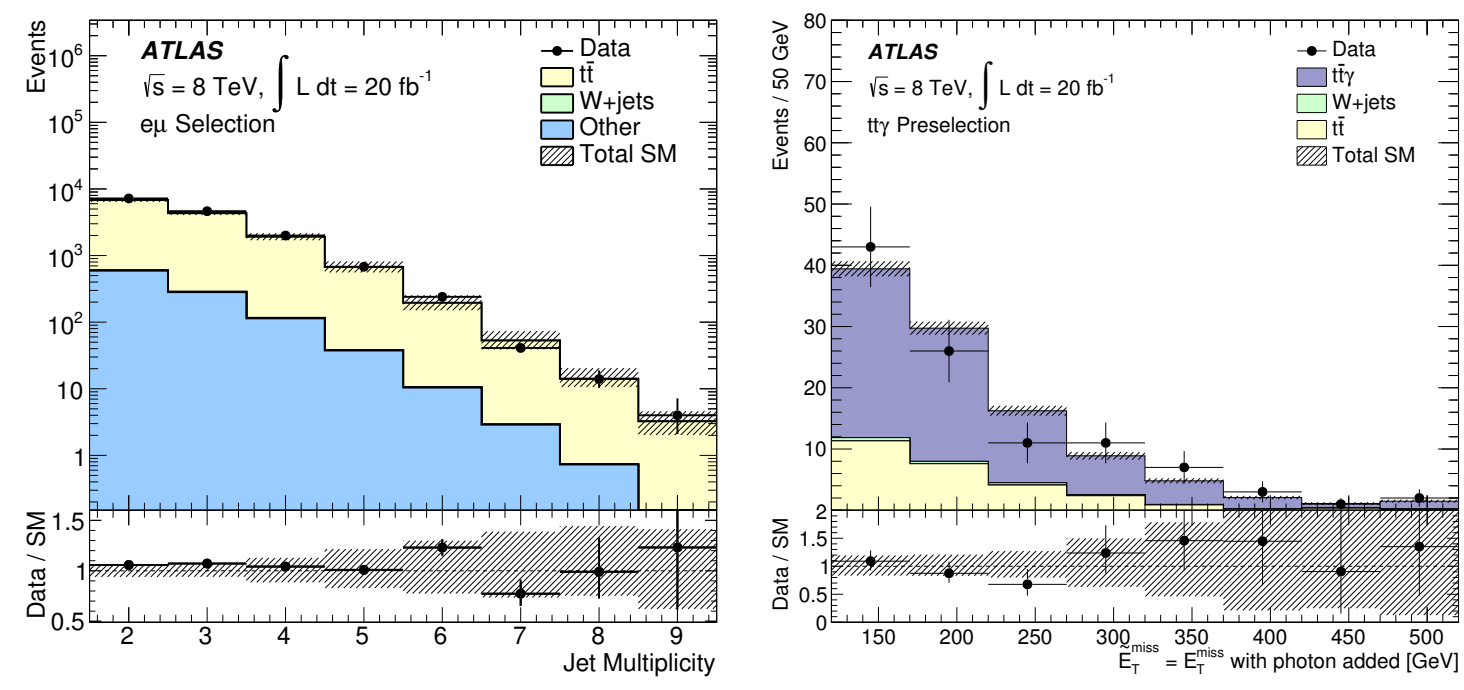

Figure 12. Left: jet multiplicity distribution for events with one opposite charged electron-muon pair and at least two jets of which one or more is $b$-tagged. Other processes include single top quark production, $t \bar{t}$ production in association with a vector boson, $Z+$ jets, and diboson production. Right: missing transverse momentum where photons are treated as invisible particles $\left(\tilde{E}_{\mathrm{T}}^{\text {miss }}\right)$ for an event selection of $t \bar{t}+$ photon (described in the text). Both plots: the uncertainty band includes all statistical and experimental uncertainties, and the last bins include overflows.

ground and signal samples. Since the yields for the dominant background sources, $t \bar{t}$ and $W+$ jets, are obtained in dedicated control regions, the modelling uncertainties for these processes affect only the extrapolation from the CRs into the signal regions (and between TCR and WCR), but not the overall normalisation. The systematic uncertainties are included as nuisance parameters and profiled in the likelihood fits. The nuisance parameters are constrained by Gaussian terms with widths corresponding to the sizes of the systematic uncertainties. The same set of nuisance parameters is used across all bins, with the exception of the two shape-fits that have three $t \bar{t}$ and three $W+$ jets normalisation parameters and hence also have three sets of nuisance parameters. The effects of the sources of uncertainties discussed in this section are quantified in terms of the corresponding relative uncertainty on the estimated number of background events in the various signal regions, this is referred to as the 'impact on the background estimate'.

The dominant experimental uncertainties arise from imperfect knowledge of the jet energy scale (JES) and jet energy resolution (JER) as well as from the modelling of the $b$-tagging efficiency. The JES uncertainty is derived from a combination of simulation and data samples $[105,106]$ taking into account the dependence on the $p_{\mathrm{T}}, \eta$ and flavour of the jet as well as the amount of pileup. The impact of JES on the background estimate varies from $1 \%$ to $13 \%$. The JER uncertainties are determined with in-situ measurements of the jet response balance in dijet events [129], and the impact on the background estimate is $1 \%-21 \%$. The JES, JER, and jet mass scale and resolution uncertainties for large- $R$ jets are derived from a combination of data and simulation samples [107, 133], and their combined impact on the background estimate amounts to $3 \%$. The $b$-tagging uncertainty is estimated 
by varying the $b$-tagging efficiency and mis-tag rate correction factors, obtained from datadriven measurements of these quantities in $t \bar{t}$ and dijet events [111, 134-136], within their uncertainties. The impact of these uncertainties on the background estimate ranges from $1 \%$ to $8 \%$, and is dominated by the uncertainty on the $b$-tagging efficiency. Other sources of experimental uncertainty are the modelling of the average number of $p p$ interactions per bunch crossing, the modelling of the contribution to the $E_{\mathrm{T}}^{\text {miss }}$ from energy deposits not associated with any reconstructed objects and from pileup, the modelling of leptonrelated quantities (trigger and identification efficiency, energy and momentum scale and resolution, isolation and $\tau$-veto) as well as imperfect knowledge of the integrated luminosity. The combined impact of these sources on the background estimate is between $1 \%$ and $5 \%$.

Uncertainties related to theoretical predictions and MC modelling are evaluated for all signal and background processes obtained entirely or partly from simulated events. The sources of uncertainty considered for both the $t \bar{t}$ and $W+$ jets background processes are the variations of the renormalisation and factorisation scales by factors of 0.5 and 2.0 as well as PDF variations, which are studied following the PDF4LHC recommendations [137] comparing CT10 NLO, MSTW2008 NNLO and NNPDF21_100 [138] PDF error sets. For the $t \bar{t}$ background, the uncertainty on the hadronisation modelling is derived from a comparison between events generated with POWHEG and interfaced with PYTHIA for the shower model and those generated in the same way, but interfaced with HeRwig-Jimmy [139]. Furthermore, the effect of the modelling of ISR and final-state radiation (FSR) is studied using samples of $t \bar{t}$ events generated with ACERMC with reduced and increased amounts of additional radiation (constrained by the measurement of ref. [140]). The impact of the $t \bar{t}$ modelling on the background estimate is $2 \%-6 \%$. For the $W+$ jets background, the effect of varying the number of partons used in the hard-scatter process is estimated by comparing samples generated with up to four extra partons to samples generated with up to five extra partons. The impact of merging matrix elements and parton showers is studied by varying the SHERPA scales related to the matching scheme. As the $W$ +jets background is normalised in a region with a $b$-tag veto, additional uncertainties on the flavour composition of the $W+$ jets events in the signal region, based on the uncertainties on the measurement of ref. [141] extrapolated to higher jet multiplicities, are applied in all regions requiring at least one $b$-tagged jet. The impact of the $W+$ jets modelling on the background estimate is $1 \%-7 \%$.

Background sources other than $t \bar{t}$ and $W+$ jets are estimated from simulated events and are normalised to the most accurate cross-section predictions available. The cross-section uncertainty for the single-top process is $7 \%$ [82-84], while it is $22 \%$ for $t \bar{t} V[85,86]$. The $Z Z$ and $W Z$ cross-section uncertainties are $5 \%$ and $7 \%$, respectively [89, 90]. Other sources of systematic uncertainty considered depend on the physics process, but include the choice of renormalisation and factorisation scales, PDF variations, hadronisation modelling, choice of MC generator, modelling of ISR and FSR, variations of the matrix element to parton shower matching scales, the generation of a finite number of partons, and the interference between single-top and $t \bar{t}$ production at NLO. The uncertainty on the interference treatment is estimated using inclusive $W W b b$ samples at LO generated with ACERMC (which includes both the $t \bar{t}$ and $W t$ processes). The total impact of the modelling of the smaller backgrounds on the background estimate ranges from $1 \%$ to $11 \%$. 
The theoretical uncertainties affecting the signal yields originate from the uncertainty on the production cross-section [96], and from the uncertainty on the acceptance. The latter includes PDF variations assessed using the PDF4LHC prescription [137], as well as modelling uncertainties of ISR and FSR and variations of the renormalisation and factorisation scales, evaluated by varying the relevant parameters in MADGRAPH. The total uncertainty on the production cross-section varies as a function of the stop mass; it amounts to about $15 \%$ for $m_{\tilde{t}_{1}}=200 \mathrm{GeV}$ and increases to $18 \%$ for $m_{\tilde{t}_{1}}=700 \mathrm{GeV}$. The impact of the ISR/FSR modelling uncertainty on the signal acceptance ranges from $10 \%$ to $20 \%$ for signal regions that select events with ISR activity, such as bCc_diag, and for signal models of mass hierarchy (c). It is negligible for the other signal regions.

The search sensitivity is directly connected to the fitted uncertainty of the signal strength parameter, where the signal strength is a fit parameter that scales the signal yield predicted by the model in question; a signal strength of one corresponds to the nominal signal yield. The impact of the various sources of uncertainty, including the statistical precision, on the signal strength uncertainty is quantified in table 10 for selected signal regions and signal benchmark models. The breakdown of the size of the systematic uncertainties is evaluated by re-running the fit, fixing the relevant nuisance parameter in question to its value from the nominal fit, and taking the difference in quadrature between the signal strength uncertainty of this fit and the nominal fit. The statistical uncertainty is obtained from re-running the fit without any systematic uncertainties, again fixing the nuisance parameters to their values from the nominal fit. The tightest signal regions, such as tN_boost, are statistically limited. Systematic uncertainties dominate the looser signal regions. Overall, the largest contributions to the systematic uncertainty on the signal strength come from JER and $t \bar{t}$ modelling. The energy scale and energy resolution of large- $R$ jets is relevant in the $t N_{-}$boost signal region.

\section{Results}

Figures 13 and 14 show comparisons between the observed data and the SM background prediction from the background-only fit with all selections applied except the requirement on the plotted variable. In all SRs, the plots indicate good compatibility between the data and the SM background. The expected distributions from representative signal benchmark models are overlaid.

Table 11 shows the number of observed events together with the predicted number of background events in the SRs using the model-independent selection of the 15 analyses. The predicted numbers of background events are obtained using the background-only fits to the number of observed events in the CRs as described in section 8. These fitted background estimates in the CRs are then used to obtain the fitted numbers of background events in the SRs by extrapolations that use transfer factors obtained with simulated events. The observed numbers of events are found to agree well with the fitted numbers of background events in all SRs.

To assess the compatibility of the SM background-only hypothesis with the observations in the SRs, a profile likelihood ratio test is performed implementing the methodology 

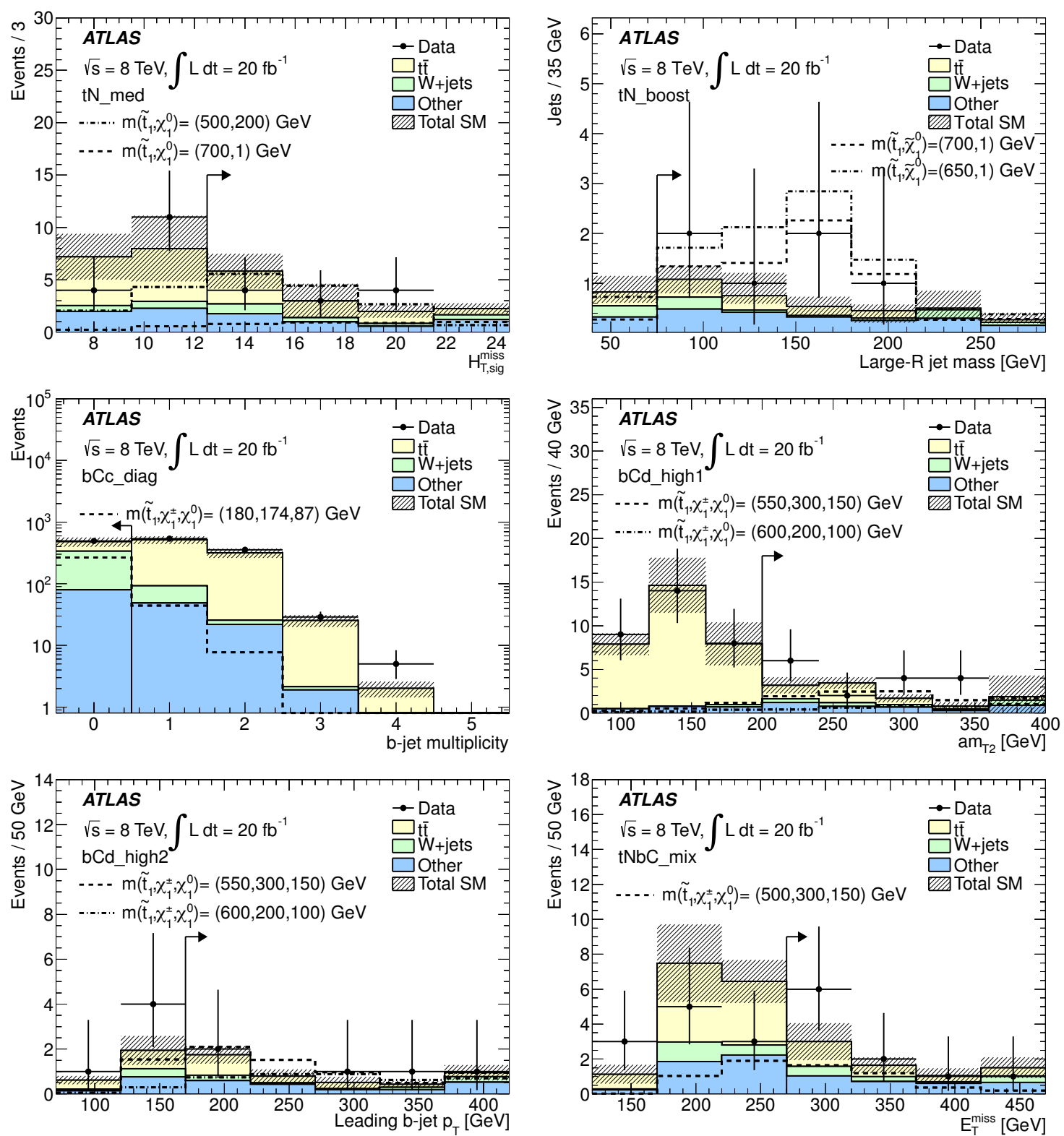

Figure 13. For each signal region one characteristic distribution is shown, with the full event selection of the signal region applied, except for the requirement (indicated by an arrow) on the shown quantity. The uncertainty band includes statistical and all experimental systematic uncertainties. The last bin includes overflows. Benchmark signal models are overlaid for comparison. 


\begin{tabular}{|l|c|c|c|c|}
\hline Uncertainty on signal strength & tN_boost & tN_diag & bCc_diag & bCd_bulk \\
\hline Total & 0.37 & 0.19 & 0.11 & 0.16 \\
\hline Statistical & 0.36 & 0.05 & 0.07 & 0.09 \\
\hline Systematic & 0.09 & 0.18 & 0.09 & 0.13 \\
\hline Contribution of systematic uncertainty components \\
\hline Jet energy scale & 0.02 & 0.03 & 0.02 & 0.03 \\
\hline Jet energy resolution & 0.06 & 0.11 & 0.06 & 0.07 \\
\hline Large- $R$-jet related & 0.03 & - & - & - \\
\hline$E_{\mathrm{T}}^{\text {miss }}$ (non-associated energy and pileup) & 0.01 & 0.06 & 0.01 & 0.03 \\
\hline Pileup & $<0.01$ & 0.03 & 0.01 & 0.02 \\
\hline$b$-tagging & 0.03 & 0.01 & 0.04 & 0.01 \\
\hline$t \bar{t}$ modelling & 0.01 & 0.15 & 0.04 & 0.08 \\
\hline$W+$ jets modelling & $<0.01$ & 0.01 & 0.02 & 0.03 \\
\hline Other backgrounds modelling & 0.04 & 0.03 & 0.02 & 0.02 \\
\hline Signal acceptance modelling & 0.02 & 0.01 & 0.02 & 0.01 \\
\hline
\end{tabular}

Table 10. Breakdown of the size of uncertainties on the signal strength parameter of the likelihood fit. The central values of the signal strength parameters (not shown) are close to zero because the data are compatible with the predicted backgrounds. The uncertainty components are obtained from the difference in quadrature between the signal strength uncertainty of the nominal fit and a fit where the systematic uncertainty in question is disabled by fixing the corresponding nuisance parameter(s) to the value(s) from the nominal fit. Some systematic uncertainty components, such as the jet energy scale or the modelling of backgrounds, are displayed as single entries while the likelihood fit employs a more detailed description. The sum in quadrature of the systematic uncertainty components may not add up to the total systematic uncertainty due to correlations. The following benchmark signal models are used: $m_{\tilde{t}_{1}}=700 \mathrm{GeV}$ and $m_{\tilde{\chi}_{1}^{0}}=1 \mathrm{GeV}$ for tN_boost, $m_{\tilde{t}_{1}}=350 \mathrm{GeV}$ and $m_{\tilde{\chi}_{1}^{0}}=150 \mathrm{GeV}$ for tN_diag $m_{\tilde{t}_{1}}=180 \mathrm{GeV}, m_{\tilde{\chi}_{1}^{ \pm}}=174 \mathrm{GeV}$ and $m_{\tilde{\chi}_{1}^{0}}=87 \mathrm{GeV}$ for bCc_diag $m_{\tilde{t}_{1}}=300 \mathrm{GeV}, m_{\tilde{\chi}_{1}^{ \pm}}=200 \mathrm{GeV}$ and $m_{\tilde{\chi}_{1}^{0}}=100 \mathrm{GeV}$ for bCd_bulk.

described in ref. [143]. The model-independent selection is used, and the likelihood for a given test includes one SR and all its associated CRs. Each SR, and each signal-sensitive bin in the two-dimensional shape-fits, is probed separately. Table 11 shows the $p_{0}$ values obtained using these fits, indicating that the data in all SRs are compatible with the background-only hypothesis. Good agreement is found when comparing the results obtained using pseudo-experiments to those calculated from asymptotic formulae [142]; the latter is used as the default for all exclusion results presented below.

As no significant excess over the expected background from SM processes is observed, the data are used to derive one-sided limits at 95\% CL. The results are obtained from a profile likelihood ratio test following the $\mathrm{CL}_{s}$ prescription [143]. Model-independent upper limits on beyond-SM contributions are derived separately for each analysis, and in case of the two-dimensional shape-fits for each signal-sensitive bin. The model-independent selection is used, and the likelihood of the fit is configured to include one SR or shape-fit bin and all its associated CRs. A generic signal model, which contributes only to the SR, 


\begin{tabular}{|c|c|c|c|c|c|c|c|}
\hline \multirow{2}{*}{ Signal region } & \multirow{2}{*}{ Obs. } & \multirow{2}{*}{ Exp. bkg. } & \multirow{2}{*}{$p_{0}$} & \multicolumn{2}{|c|}{$N_{\text {non-SM }}$} & \multicolumn{2}{|c|}{$\sigma_{\text {vis }}[\mathrm{fb}]$} \\
\hline & & & & Obs. & Exp. & Obs. & Exp. \\
\hline tN_med & 12 & $13.0 \pm 2.2$ & $\geq 0.5$ & 8.5 & 9.2 & 0.4 & 0.5 \\
\hline tN_high & 5 & $5.0 \pm 1.0$ & $\geq 0.5$ & 6.0 & 6.0 & 0.3 & 0.3 \\
\hline tN_boost & 5 & $3.3 \pm 0.7$ & 0.17 & 7.0 & 5.3 & 0.3 & 0.3 \\
\hline bCa_low & 11 & $6.5 \pm 1.4$ & 0.08 & 12.2 & 7.8 & 0.61 & 0.92 \\
\hline bCa_med & 20 & $17 \pm 4$ & 0.33 & 14.4 & 12.3 & 0.72 & 0.68 \\
\hline bCb_med1 & 41 & $32 \pm 5$ & 0.12 & 23.5 & 16.0 & 1.17 & 0.88 \\
\hline bCb_high & 7 & $9.8 \pm 1.6$ & $\geq 0.5$ & 6.5 & 7.9 & 0.32 & 0.22 \\
\hline bCc_diag & 493 & $470 \pm 50$ & 0.27 & 110.6 & 95.1 & 5.4 & 4.7 \\
\hline bCd_high1 & 16 & $11.0 \pm 1.5$ & 0.09 & 13.2 & 8.5 & 0.7 & 0.4 \\
\hline bCd_high2 & 5 & $4.4 \pm 0.8$ & 0.36 & 6.3 & 5.7 & 0.3 & 0.3 \\
\hline tNbC_mix & 10 & $7.2 \pm 1.0$ & 0.13 & 9.7 & 7.0 & 0.5 & 0.3 \\
\hline tN_diag & & & & & & & \\
\hline $125<E_{\mathrm{T}}^{\text {miss }}<150 \mathrm{GeV}, 120<m_{\mathrm{T}}<140 \mathrm{GeV}$ & 117 & $136 \pm 22$ & $\geq 0.5$ & 42.1 & 55.7 & 2.1 & 2.7 \\
\hline $125<E_{\mathrm{T}}^{\text {miss }}<150 \mathrm{GeV}, \quad m_{\mathrm{T}}>140 \mathrm{GeV}$ & 163 & $152 \pm 20$ & 0.35 & 55.4 & 47.8 & 2.7 & 2.4 \\
\hline$E_{\mathrm{T}}^{\text {miss }}>150 \mathrm{GeV}, 120<m_{\mathrm{T}}<140 \mathrm{GeV}$ & 101 & $98 \pm 13$ & 0.43 & 36.1 & 33.9 & 1.8 & 1.7 \\
\hline$E_{\mathrm{T}}^{\text {miss }}>150 \mathrm{GeV}, \quad m_{\mathrm{T}}>140 \mathrm{GeV}$ & 217 & $236 \pm 29$ & $\geq 0.5$ & 58.7 & 71.4 & 2.9 & 3.5 \\
\hline bCb_med2 & & & & & & & \\
\hline $175<a m_{\mathrm{T} 2}<250 \mathrm{GeV}, \quad 90<m_{\mathrm{T}}<120 \mathrm{GeV}$ & 10 & $12.1 \pm 2.0$ & $\geq 0.5$ & 7.3 & 8.8 & 0.4 & 0.4 \\
\hline $175<a m_{\mathrm{T} 2}<250 \mathrm{GeV}$ & 10 & $7.4 \pm 1.4$ & 0.10 & 9.7 & 7.3 & 0.5 & 0.4 \\
\hline$a m_{\mathrm{T} 2}>250 \mathrm{GeV}, \quad 90<m_{\mathrm{T}}<120 \mathrm{GeV}$ & 16 & $21 \pm 4$ & $\geq 0.5$ & 9.3 & 12.3 & 0.5 & 0.6 \\
\hline$a m_{\mathrm{T} 2}>250 \mathrm{GeV}$ & 9 & $9.1 \pm 1.6$ & $\geq 0.5$ & 7.7 & 7.8 & 0.4 & 0.4 \\
\hline bCd_bulk & & & & & & & \\
\hline $175<a m_{\mathrm{T} 2}<250 \mathrm{GeV}, \quad 90<m_{\mathrm{T}}<120 \mathrm{GeV}$ & 144 & $133 \pm 22$ & 0.29 & 36.1 & 33.9 & 1.8 & 1.7 \\
\hline $175<a m_{\mathrm{T} 2}<250 \mathrm{GeV}$ & 78 & $73 \pm 8$ & 0.34 & 58.7 & 71.4 & 2.9 & 3.5 \\
\hline $90<m_{\mathrm{T}}<120 \mathrm{GeV}$ & 61 & $66 \pm 6$ & $\geq 0.5$ & 17.5 & 20.9 & 0.9 & 1.0 \\
\hline$a m_{\mathrm{T} 2}>250 \mathrm{GeV}$ & 29 & $26.5 \pm 2.6$ & 0.34 & 14.8 & 12.6 & 0.7 & 0.6 \\
\hline 3body & & & & & & & \\
\hline $80<a m_{\mathrm{T} 2}<90 \mathrm{GeV}$ & 12 & $16.9 \pm 2.8$ & $\geq 0.5$ & 7.3 & 9.9 & 0.4 & 0.5 \\
\hline $80<a m_{\mathrm{T} 2}<90 \mathrm{GeV}$ & 8 & $8.4 \pm 2.2$ & $\geq 0.5$ & 7.9 & 7.8 & 0.4 & 0.4 \\
\hline $90<a m_{\mathrm{T} 2}<100 \mathrm{GeV}$ & 29 & $35 \pm 4$ & $\geq 0.5$ & 11.7 & 14.7 & 0.6 & 0.7 \\
\hline $90<a m_{\mathrm{T} 2}<100 \mathrm{GeV}$ & 22 & $29 \pm 5$ & $\geq 0.5$ & 55.4 & 47.8 & 2.7 & 2.4 \\
\hline
\end{tabular}

Table 11. Columns two to four show the numbers of observed events in the SRs (model-independent selection) of the 15 analyses together with the expected numbers of background events (as predicted by the background-only fits) and the probabilities, represented by the $p_{0}$ values, that the observed numbers of events are compatible with the background-only hypothesis. The $p_{0}$ values are obtained with pseudo-experiments with the exception of the shape-fit bins where only the smallest $p_{0}$ is derived with pseudo-experiments while the others are calculated from asymptotic formulae [142]. The $p_{0}$ value is set to 0.5 whenever the number of observed events is below the number of expected events. Columns five to eight show the $95 \%$ CL upper limits on the number of beyond-SM events $\left(N_{\text {non-SM }}\right)$ and on the visible signal cross-section $\left(\sigma_{\text {vis }}=\sigma_{\text {prod }} \times A \times \epsilon\right)$. The observed and (median) expected limits are given for a generic model without uncertainties other than on the luminosity. 

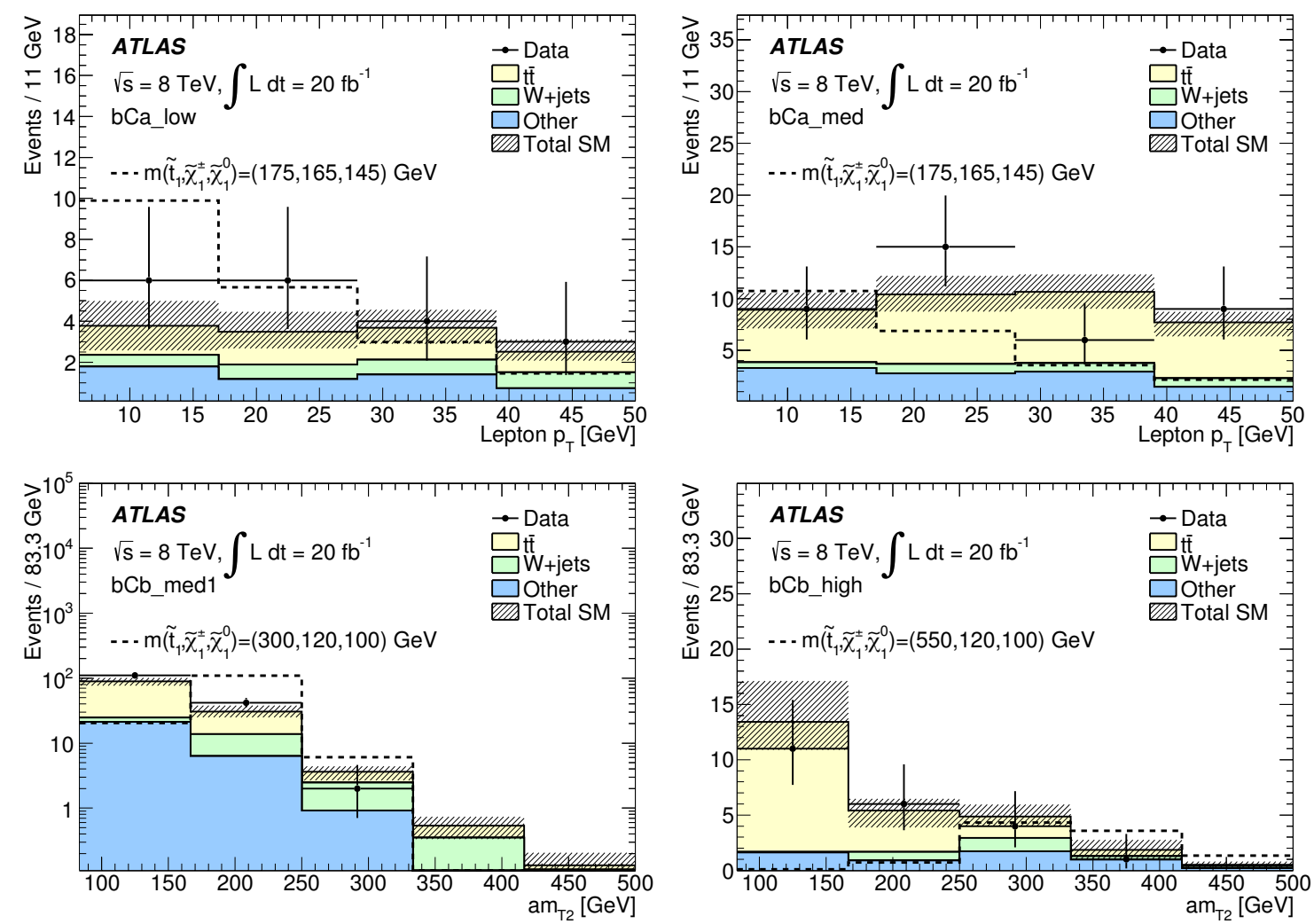

Figure 14. For each signal region one characteristic distribution is shown, with the full event selection of the signal region applied, except for the requirement on the shown quantity. The binning is similar to that used for the one-dimensional shape-fits of the corresponding analyses. The uncertainty band includes statistical and all experimental systematic uncertainties. The last bin includes overflows. Benchmark signal models are overlaid for comparison.

is assumed and no experimental or theoretical signal systematic uncertainties are assigned other than the luminosity uncertainty. The resulting limits on the number of beyondSM events and on the visible signal cross-section are shown in the rightmost columns of table 11. The visible signal cross-section $\left(\sigma_{\text {vis }}\right)$ is defined as the product of acceptance $(A)$, reconstruction efficiency $(\epsilon)$ and production cross-section $\left(\sigma_{\text {prod }}\right)$; it is obtained by dividing the upper limit on the number of beyond-SM events by the integrated luminosity.

Exclusion limits are also derived in various SUSY scenarios. The results are obtained using the same $\mathrm{CL}_{s}$ prescription as used for the model-independent limits, but with the model-dependent selection. The likelihood for each analysis includes the full set of bins: SR, TCR, WCR for cut-and-count and the full set of SR and CR bins for shape-fit analyses. The signal uncertainties and potential signal contributions to all bins are taken into account. All uncertainties except on the theoretical signal cross-section are included in the fit. Combined exclusion limits are obtained by selecting a priori the signal region with the lowest expected $\mathrm{CL}_{s}$ value for each signal grid point.

The expected and observed exclusion contours for the $\tilde{t}_{1} \rightarrow t \tilde{\chi}_{1}^{0}$ decay mode are shown in figure 15 overlaying the results for the signal regions targeting two-, three- and four- 
body decays. The $\pm 1 \sigma_{\text {exp }}$ uncertainty band indicates the impact on the expected limit of

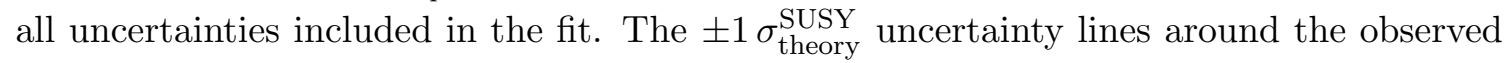
limit illustrate the change in the observed limit as the nominal signal cross-section is scaled up and down by the theoretical cross-section uncertainty. Quoted limits are derived from

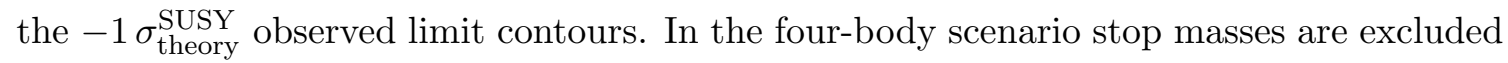
between 100 and $170 \mathrm{GeV}$, for an LSP mass of about $75 \mathrm{GeV}$. Stop masses between 100 and nearly $175 \mathrm{GeV}$ in the three-body scenario, and between 210 and $640 \mathrm{GeV}$ in the twobody scenario are excluded for a massless LSP, while for a stop mass around $550 \mathrm{GeV}$ the exclusion reaches up to an LSP mass of $230 \mathrm{GeV}$. The non-excluded area between the four- and three-body decay regions is due to a reduction in search sensitivity as the kinematic properties of the signal change significantly when transitioning from a fourbody to a three-body decay. In particular, approaching this boundary from the threebody side, the momenta of the two $b$-jets decrease to zero and hence the acceptance of the $p_{\mathrm{T}}$ requirement on the $b$-tagged jet in the 3 body signal region drops quickly. The kinematic properties change again at the other diagonal, between the three-body and onshell top quark decay modes. When approaching this diagonal from the on-shell top quark side the search sensitivity is limited by the difficulty to disentangle the signal from the $t \bar{t}$ background, as the two processes begin to closely resemble each other in kinematic properties. In the limit of reaching the diagonal from the righthand side, the two LSPs have no phase space, thus carrying away no momentum, leading to a stop signature similar to that of $t \bar{t}$ except for small deviations induced by the difference in spin. This region is also referred to as 'stealth stop'. The tN_diag signal region has the best expected sensitivity for stop masses up to $400 \mathrm{GeV}$ and close to the $m_{\tilde{t}_{1}} \gtrsim m_{t}+m_{\tilde{\chi}_{1}^{0}}$ kinematic boundary, while the tN_boost signal region has the best expected sensitivity for stop masses above $600 \mathrm{GeV}$. In the intermediate mass region the best expected sensitivity comes from the $\mathrm{tN}$ med signal region. The use of large- $\mathrm{R}$ jets in the $\mathrm{tN}_{-}$boost signal region extends the reach for a heavy stop by about $30 \mathrm{GeV}$, as obtained from a comparison with the tN_high signal region.

Figures 16 to 21 show the expected and observed exclusion contours for the $\tilde{t}_{1} \rightarrow b \tilde{\chi}_{1}^{ \pm}$ decay mode with different $\tilde{\chi}_{1}^{ \pm}$mass hypotheses. If the mass of the $\tilde{\chi}_{1}^{ \pm}$is twice that of the LSP (figure 16), stop masses up to $500 \mathrm{GeV}$ are excluded for an LSP mass in the range of 100 to $150 \mathrm{GeV}$. The various regions in the exclusion area can be mapped to the mass hierarchies illustrated in figure 4: models in the bulk region correspond to mass hierarchy (d), those in the (bottom) region with a low $\tilde{\chi}_{1}^{0}$ mass are mass hierarchy (b), and the ones in the (diagonal) region close to the kinematic boundary are mass hierarchy (c). The strongest exclusion sensitivity is provided by the signal regions designed for the given mass hierarchy, for example bCd_bulk (bulk), bCb_med1 and bCb_high (bottom), and bCc_diag (diagonal). The small region around $m_{\tilde{t}_{1}} \sim 175 \mathrm{GeV}$ and $m_{\tilde{\chi}_{1}^{0}} \lesssim 70 \mathrm{GeV}$ is not excluded because signal events cannot be sufficiently well distinguished from $t \bar{t}$ background events; for larger $m_{\tilde{\chi}_{1}^{0}}$ values, the $b$-tag veto in the bCc_diag signal region becomes effective.

If the $\tilde{\chi}_{1}^{ \pm}$mass is set to $150 \mathrm{GeV}$ (figure 17), stop masses below $490 \mathrm{GeV}$ are excluded for LSP masses up to $80 \mathrm{GeV}$. Models in the top, left, and bulk regions within the exclusion 
contour correspond to the mass hierarchies (a-b), (c), and (d) respectively; the exclusion power is mostly provided by bCb_med1 and bCb_high (top), bCc_diag (left), bCd_bulk (bulk), and bCb_med2 (bulk with high $m_{\tilde{t}_{1}}$ ). The vertical drop in search sensitivity for $m_{\tilde{t}_{1}} \lesssim 260 \mathrm{GeV}$ in the top part of the plane is caused by the $b$-tagged jet $p_{\mathrm{T}}$ and $m_{b b}$ requirements in the two $\mathrm{bCb}_{-} *$ signal regions, which effectively imposes a minimum mass splitting between the $\tilde{t}_{1}$ and $\tilde{\chi}_{1}^{ \pm}$states. The other two signal regions that are based on softlepton selections $\left(\mathrm{bCa}_{-} *\right)$ require ISR activity and are hence limited to the low stop mass region where the cross-section is large. The sharp horizontal contour line at $m_{\tilde{\chi}_{1}^{0}}=145 \mathrm{GeV}$ is an artifact caused by the fact that signal grid points were generated only up to this LSP mass. However, the upper region is excluded by the search described in ref. [37]. If the mass of the $\tilde{\chi}_{1}^{ \pm}$is set to $106 \mathrm{GeV}$ (figure 18), stop masses between 240 and $550 \mathrm{GeV}$, together with a small region around a stop mass of $150 \mathrm{GeV}$, are excluded for an LSP mass of $70 \mathrm{GeV}$. The identification of regions with mass hierarchies, and the best signal regions are similar to the previous scenario. The vertical exclusion gap around $m_{\tilde{t}_{1}} \sim 175 \mathrm{GeV}$ is due to the same effect seen in figure 16 and described above, while the reduction in exclusion power for a decreasing LSP mass is due to the transition of the $\tilde{\chi}_{1}^{ \pm}$decay from a three-body to a two-body process, which happens at an LSP mass of $\sim 26 \mathrm{GeV}$; the two-body decay produces less $E_{\mathrm{T}}^{\text {miss }}$ on average, amongst other changes of the kinematic properties.

If the $\tilde{\chi}_{1}^{ \pm}$mass is only $5 \mathrm{GeV}(20 \mathrm{GeV})$ above the LSP mass (figures 19 and 20, respectively), stop masses between 265 and $600 \mathrm{GeV}$ (240 and $600 \mathrm{GeV}$ ) are excluded for an LSP mass of $100 \mathrm{GeV}$. The exclusion in these 'compressed' scenarios, corresponding to the mass hierarchies (a) and (b), is achieved using the soft-lepton selections of the bCa_low and $\mathrm{bCa}$ med signal regions. The sensitivity decreases for smaller mass splittings because of the lepton $p_{\mathrm{T}}$ threshold. The diagonal exclusion gap in figure 20 is caused by the effects described above in the discussion of figure 17 together with the impact of the $b$-veto on the leading jet in the bCa_* signal regions. If the $\tilde{\chi}_{1}^{ \pm}$mass is only $10 \mathrm{GeV}$ below the stop mass (figure 21), stop masses below $390 \mathrm{GeV}$ are excluded for a massless LSP. The models in this scenario correspond to mass hierarchy (c). The bulk exclusion power comes from the bCc_diag signal region with a $b$-veto, while using soft leptons in the bCa_low signal region extends the sensitivity in the top left region.

The complementarity of the signal regions to various mass splittings of $\tilde{\chi}_{1}^{ \pm}$and $\tilde{\chi}_{1}^{0}$ is illustrated by fixing the stop mass to $300 \mathrm{GeV}$ and presenting the exclusion limit as a function of the $\tilde{\chi}_{1}^{ \pm}$and $\tilde{\chi}_{1}^{0}$ masses (figure 22). LSP masses up to about $100 \mathrm{GeV}$ are excluded for all possible $\tilde{\chi}_{1}^{ \pm}$masses, with one small exception in the bottom left corner. The exclusion power close to the kinematic boundary comes from the signal regions designed for mass hierarchy (a) and (b), while for larger mass splittings the sensitivity is provided by the selections for mass hierarchy $(\mathrm{d})$, and (c) in case of a large $\tilde{\chi}_{1}^{ \pm}$mass. The non-excluded region around a $\tilde{\chi}_{1}^{ \pm}$mass of $270 \mathrm{GeV}$ and a $\tilde{\chi}_{1}^{0}$ mass of $175 \mathrm{GeV}$ is caused by the signal region transition.

In scenarios where both the $\tilde{t}_{1} \rightarrow t \tilde{\chi}_{1}^{0}$ and $\tilde{t}_{1} \rightarrow b \tilde{\chi}_{1}^{ \pm}$decay modes are allowed and where $m_{\tilde{\chi}_{1}^{ \pm}}=2 m_{\tilde{\chi}_{1}^{0}}$ (figure 23), the largest excluded stop mass for an LSP mass of 
$100 \mathrm{GeV}$ gradually increases from $530 \mathrm{GeV}$ to $660 \mathrm{GeV}$ as the branching ratio for $\tilde{t}_{1} \rightarrow t \tilde{\chi}_{1}^{0}$ is increased from $0 \%$ to $100 \%$. Here, the quoted limits correspond to the central observed limit contour. The signal regions providing the best expected sensitivity for models with mixed decays are mainly bCd_bulk and tNbC_mix, with some contribution from tN_diag, $\mathrm{tN}$ med and $\mathrm{tN} \_$boost for models with a large fraction of $\tilde{t}_{1} \rightarrow t \tilde{\chi}_{1}^{0}$ decays, as well as some contributions from $\mathrm{bCb}$ med1 and bCb_high for models with a low LSP mass and a small fraction of $\tilde{t}_{1} \rightarrow t \tilde{\chi}_{1}^{0}$ decays.

The upper limits on the signal cross-section for models where the $\tilde{t}_{1}$ is a pure $\tilde{t}_{\mathrm{L}}$ and models where it is predominantly a $\tilde{t}_{\mathrm{R}}$ are compared in figure 24 for the $\tilde{t}_{1} \rightarrow t \tilde{\chi}_{1}^{0}$ decay mode with two assumptions for the lightest neutralino, $m_{\tilde{\chi}_{1}^{0}}=50 \mathrm{GeV}$ and $m_{\tilde{\chi}_{1}^{0}}=150 \mathrm{GeV}$. The predominantly $\tilde{t}_{\mathrm{R}}$ mixing composition is the default setting used for all simplifiedmodel $\tilde{t}_{1} \rightarrow t \tilde{\chi}_{1}^{0}$ scenarios. The weaker $\tilde{t}_{\mathrm{L}}$ model exclusion is mainly the result of a reduced lepton and $m_{\mathrm{T}}$ acceptance. The excluded $\tilde{t}_{1}$ mass reach of the $\tilde{t}_{\mathrm{L}}$ model is reduced by about $50 \mathrm{GeV}$ for the two considered LSP masses.

The change in sensitivity when varying parameters other than the stop and $\tilde{\chi}_{1}^{0}$ masses is studied using 27 pMSSM samples, which can be classified into three groups of similar stop and $\tilde{\chi}_{1}^{0}$ masses; a detailed description is given in section 5.2. The expected and observed $\mathrm{CL}_{s}$ significance values for the $27 \mathrm{pMSSM}$ models and two simplified models are shown in figure 25. The strongest impact on the $\mathrm{CL}_{s}$ significance is found to be from the sum of the branching ratios for $\tilde{t}_{1} \rightarrow t \tilde{\chi}_{1}^{0}$ and $\tilde{t}_{1} \rightarrow b \tilde{\chi}_{1}^{ \pm}$, where the $\mathrm{CL}_{s}$ significance is smaller for models where stop decays other than $\tilde{t}_{1} \rightarrow t \tilde{\chi}_{1}^{0}$ and $\tilde{t}_{1} \rightarrow b \tilde{\chi}_{1}^{ \pm}$are kinematically allowed. This is a consequence of the signal selections being optimised using only simplified models. In addition to the branching ratio dependence, the sensitivity also depends on the kinematic properties of the events, which are affected, e.g., by the stop mixing matrix and by the masses and field content of other SUSY particles. These additional dependencies explain the large spread in $\mathrm{CL}_{s}$ significance for the models where the stop decays only to $t \tilde{\chi}_{1}^{0}$ and $b \tilde{\chi}_{1}^{ \pm}$. For $m_{\tilde{t}_{1}} \sim 400 \mathrm{GeV}$ and $m_{\tilde{\chi}_{1}^{0}} \sim 50 \mathrm{GeV}, 11$ of the 14 models are excluded. No models with $m_{\tilde{t}_{1}} \sim 550 \mathrm{GeV}$ are excluded.

\section{Summary and conclusions}

A search for stop pair production in final states with one isolated lepton, jets, and missing transverse momentum is presented. Proton-proton collision data from the full 2012 datataking period were analysed, corresponding to an integrated luminosity of $20 \mathrm{fb}^{-1}$ collected at $\sqrt{s}=8 \mathrm{TeV}$ by the ATLAS detector at the LHC. Five decay modes are considered: (1) each stop decays to a top quark and the LSP; (2) each stop decays to a bottom quark and the lightest chargino $\left(\tilde{\chi}_{1}^{ \pm}\right)$, where the $\tilde{\chi}_{1}^{ \pm}$decays via an on- or off-shell $W$ boson to the LSP; (3) each stop decays in a three-body process to a bottom quark, a $W$ boson, and the LSP; (4) each stop decays in a four-body process to a bottom quark, the LSP and two light fermions; (5) the two stops decay independently either as described in (1) or in (2). In all scenarios, $R$-parity is conserved and the LSP is assumed to be the $\tilde{\chi}_{1}^{0}$.

The results are in agreement with predictions from the Standard Model, and are thus translated into $95 \%$ CL upper limits on the stop and $\tilde{\chi}_{1}^{0}$ masses in various supersymmetric 


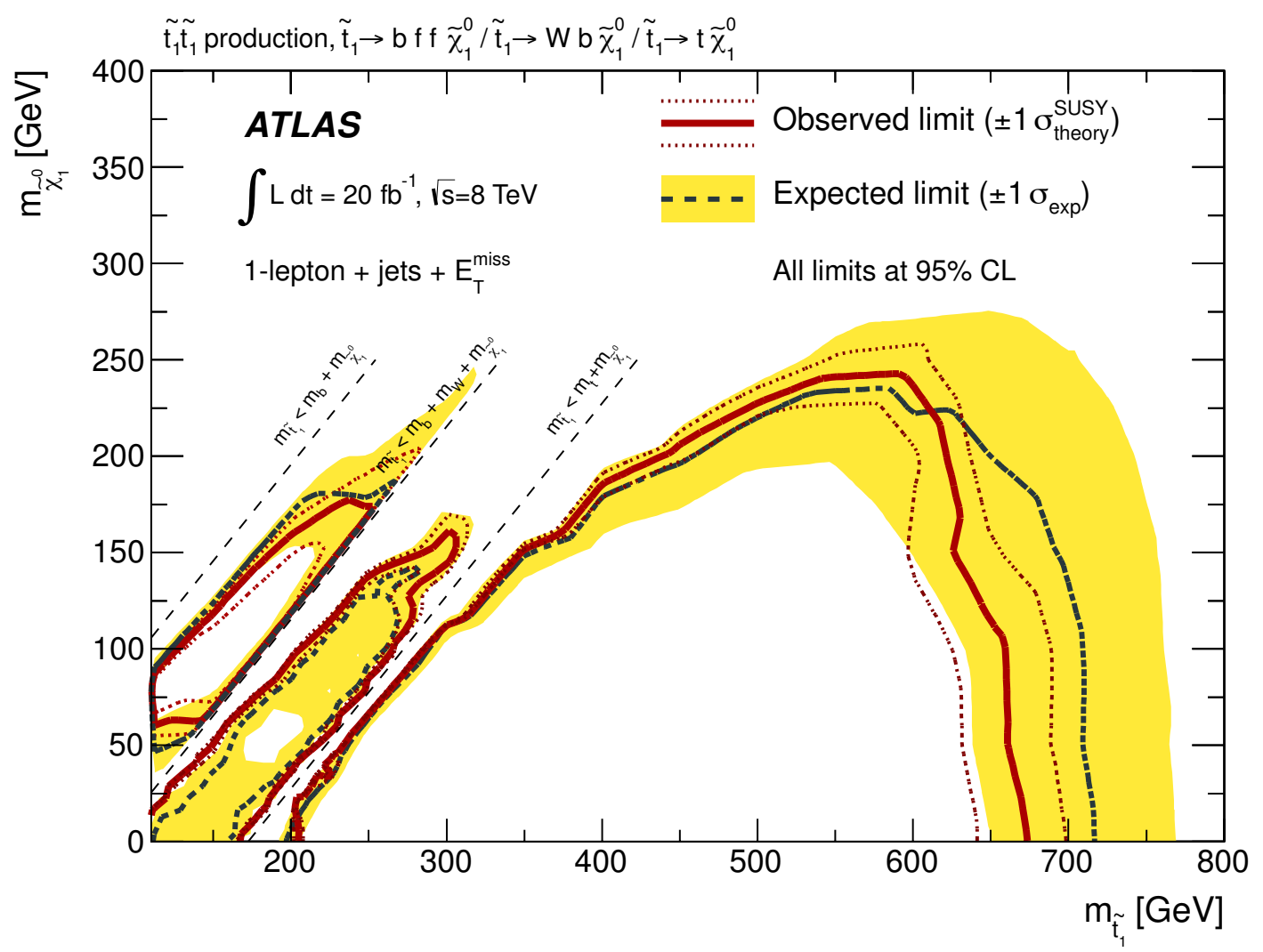

Figure 15. Expected (black dashed) and observed (red solid) 95\% CL excluded region in the plane of $m_{\tilde{\chi}_{1}^{0}}$ vs. $m_{\tilde{t}_{1}}$, assuming $\mathcal{B R}\left(\tilde{t}_{1} \rightarrow t \tilde{\chi}_{1}^{0}\right)=100 \%$. In the region $m_{b}+m_{W}+m_{\tilde{\chi}_{1}^{0}}<m_{\tilde{t}_{1}}<m_{t}+m_{\tilde{\chi}_{1}^{0}}$ the decay of the $\tilde{t}_{1}$ involves a virtual top quark (three-body decay), while in the region $m_{\tilde{t}_{1}}<m_{b}+$ $m_{W}+m_{\tilde{\chi}_{1}^{0}}$ it involves both a virtual top quark and a virtual $W$ boson (four-body decay). The $m_{\tilde{t}_{1}}<$ $100 \mathrm{GeV}$ region for the three-body decay mode is excluded by the search described in ref. [38]. Furthermore, the $m_{\tilde{t}_{1}}<78 \mathrm{GeV}$ region in the four-body scenario is excluded by the search in ref. [144].

scenarios. For models where the stop decays exclusively into a top quark and a $\tilde{\chi}_{1}^{0}$ (scenario (1) above), stop masses between 210 and $640 \mathrm{GeV}$ are excluded for a massless LSP, and stop masses around $550 \mathrm{GeV}$ are excluded for LSP masses below $230 \mathrm{GeV}$. Limits are also derived in the three- and four-body scenarios. For scenarios where the stop decays exclusively into a bottom quark and a $\tilde{\chi}_{1}^{ \pm}$(scenario (2) above), the excluded stop and $\tilde{\chi}_{1}^{0}$ masses depend strongly on the mass of the $\tilde{\chi}_{1}^{ \pm}$. For models where the mass of the $\tilde{\chi}_{1}^{ \pm}$is twice that of the LSP, stop masses up to $500 \mathrm{GeV}$ are excluded for an LSP mass in the range of 100 to $150 \mathrm{GeV}$. For models in which the $\tilde{\chi}_{1}^{ \pm}$mass is only $20 \mathrm{GeV}$ above the LSP mass, stop masses between 240 and $600 \mathrm{GeV}$ are excluded for an LSP mass of $100 \mathrm{GeV}$. In scenarios where only the $\tilde{t}_{1} \rightarrow t \tilde{\chi}_{1}^{0}$ and $\tilde{t}_{1} \rightarrow b \tilde{\chi}_{1}^{ \pm}$decay modes are allowed, the largest excluded stop mass for an LSP mass of $100 \mathrm{GeV}$ gradually increases from $530 \mathrm{GeV}$ to $660 \mathrm{GeV}$ as the branching ratio for $\tilde{t}_{1} \rightarrow t \tilde{\chi}_{1}^{0}$ is increased from $0 \%$ to $100 \%$. Using a limited set of pMSSM models, the exclusion power is found to decrease with an increased branching ratio to decays other than $\tilde{t}_{1} \rightarrow$ $t \tilde{\chi}_{1}^{0}$ and $\tilde{t}_{1} \rightarrow b \tilde{\chi}_{1}^{ \pm}$. These results supersede and significantly extend previous ATLAS limits. 


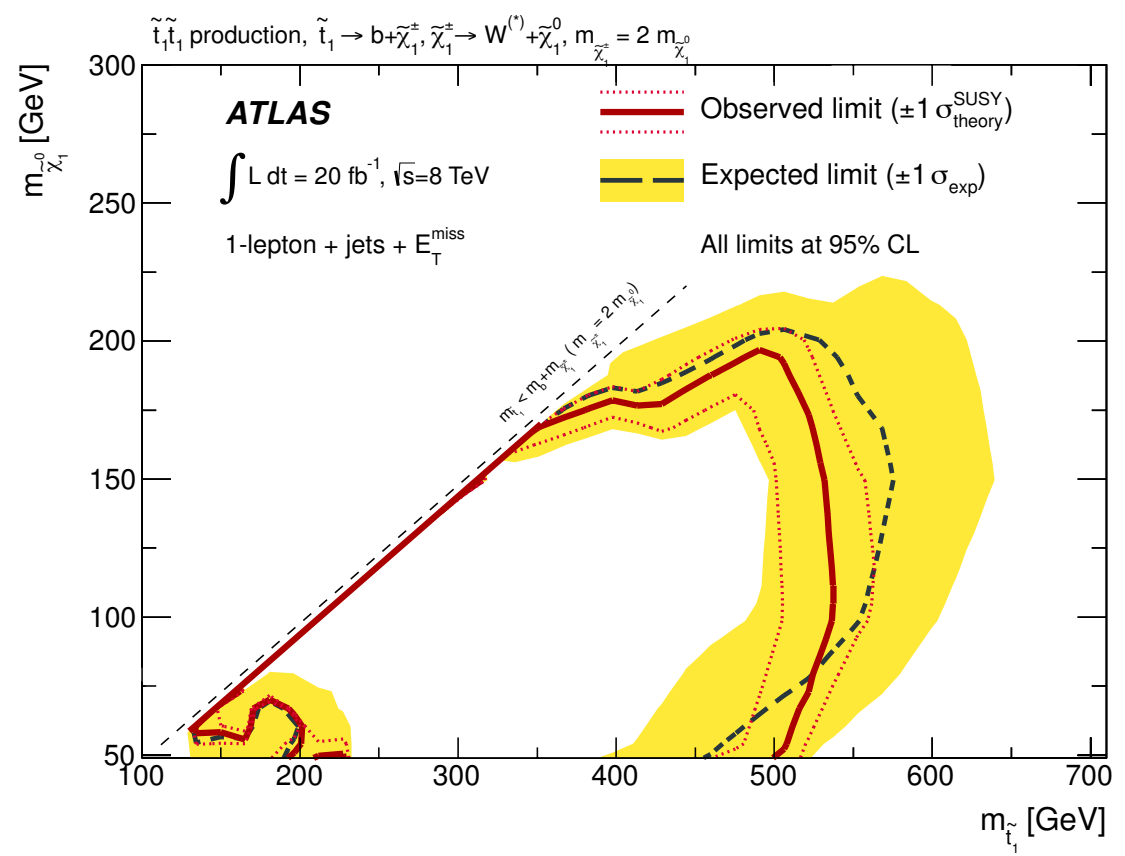

Figure 16. Expected (black dashed) and observed (red solid) 95\% CL excluded region in the plane of $m_{\tilde{\chi}_{1}^{0}}$ vs. $m_{\tilde{t}_{1}}$, assuming $\mathcal{B R}\left(\tilde{t}_{1} \rightarrow b \tilde{\chi}_{1}^{ \pm}\right)=100 \%, \mathcal{B R}\left(\tilde{\chi}_{1}^{ \pm} \rightarrow W^{(\star)} \tilde{\chi}_{1}^{0}\right)=100 \%$ and $m_{\tilde{\chi}_{1}^{ \pm}}=2 m_{\tilde{\chi}_{1}^{0}}$.

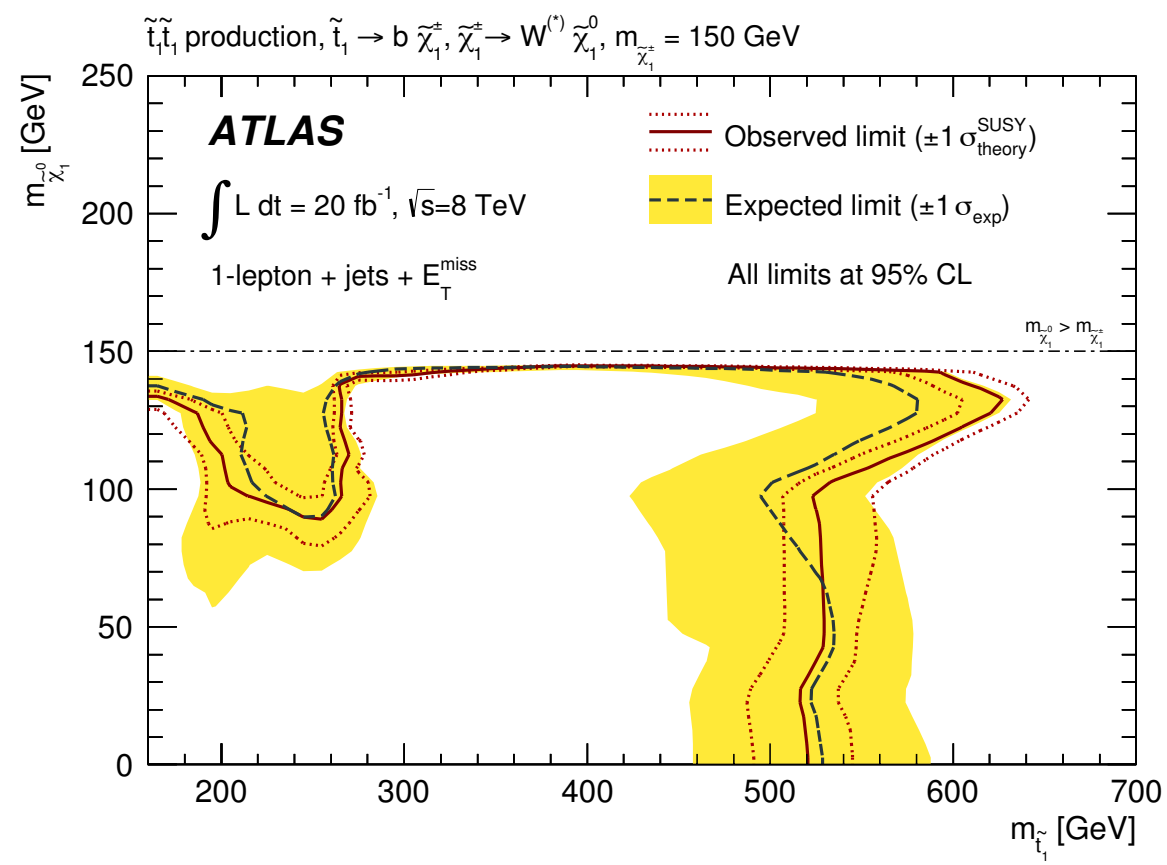

Figure 17. Expected (black dashed) and observed (red solid) 95\% CL excluded region in the plane of $m_{\tilde{\chi}_{1}^{0}}$ vs. $m_{\tilde{t}_{1}}$, assuming $\mathcal{B R}\left(\tilde{t}_{1} \rightarrow b \tilde{\chi}_{1}^{ \pm}\right)=100 \%, \mathcal{B R}\left(\tilde{\chi}_{1}^{ \pm} \rightarrow W^{(\star)} \tilde{\chi}_{1}^{0}\right)=100 \%$ and $m_{\tilde{\chi}_{1}^{ \pm}}=150 \mathrm{GeV}$. 


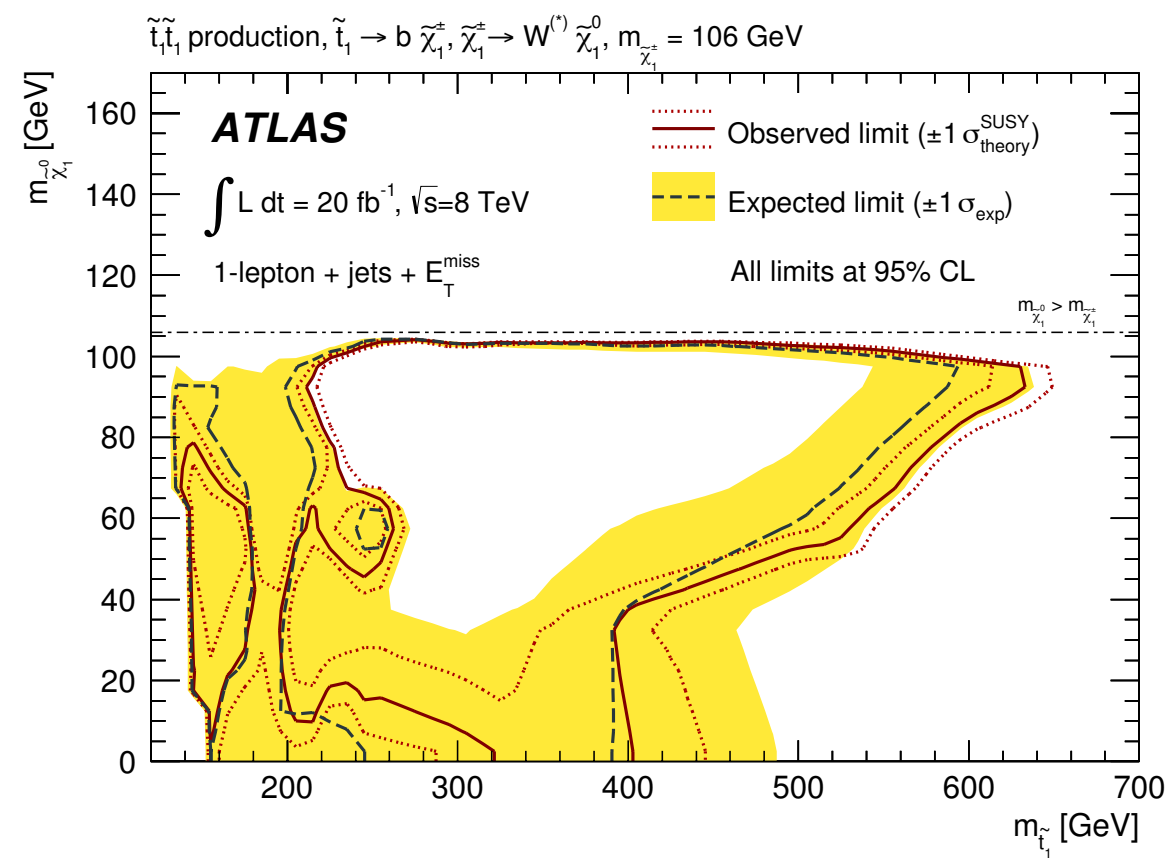

Figure 18. Expected (black dashed) and observed (red solid) 95\% CL excluded region in the plane of $m_{\tilde{\chi}_{1}^{0}}$ vs. $m_{\tilde{t}_{1}}$, assuming $\mathcal{B R}\left(\tilde{t}_{1} \rightarrow b \tilde{\chi}_{1}^{ \pm}\right)=100 \%, \mathcal{B R}\left(\tilde{\chi}_{1}^{ \pm} \rightarrow W^{(\star)} \tilde{\chi}_{1}^{0}\right)=100 \%$ and $m_{\tilde{\chi}_{1}^{ \pm}}=106 \mathrm{GeV}$.

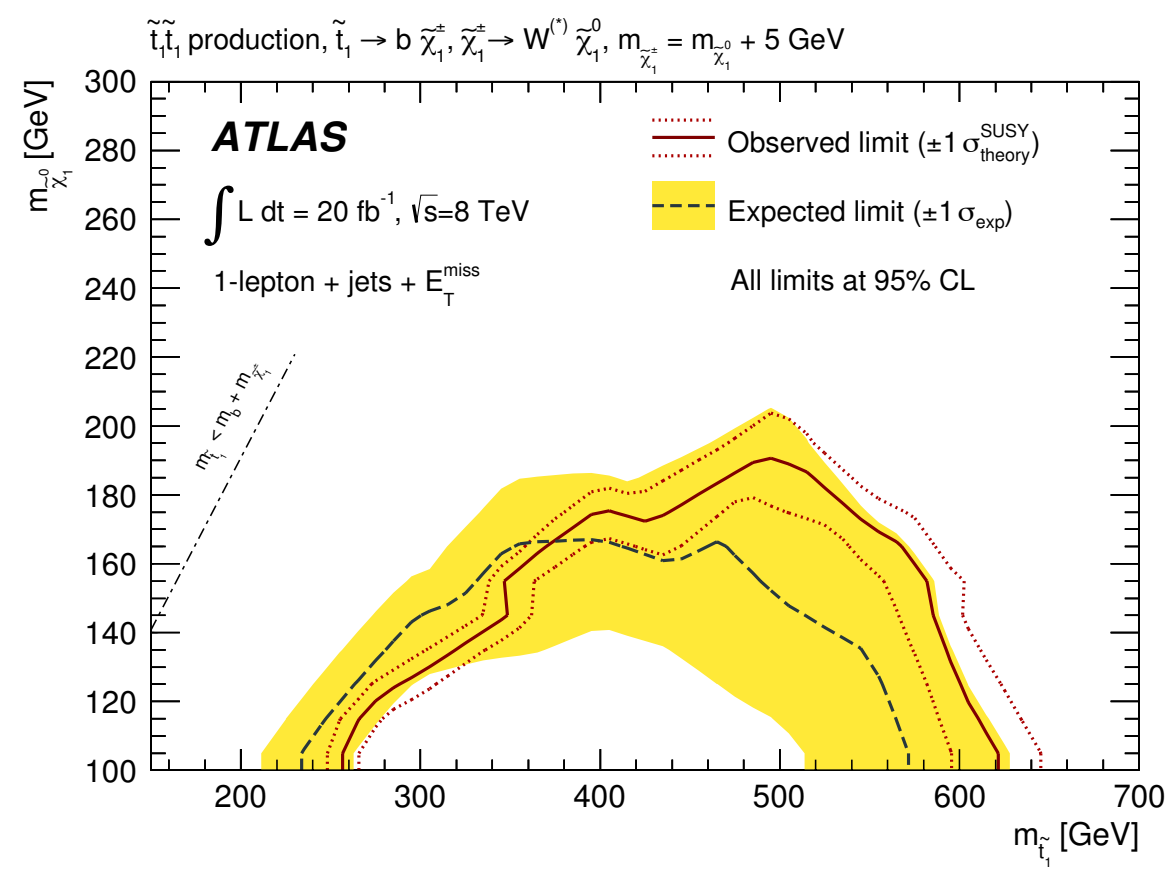

Figure 19. Expected (black dashed) and observed (red solid) 95\% CL excluded region in the plane of $m_{\tilde{\chi}_{1}^{0}}$ vs. $m_{\tilde{t}_{1}}$, assuming $\mathcal{B R}\left(\tilde{t}_{1} \rightarrow b \tilde{\chi}_{1}^{ \pm}\right)=100 \%, \mathcal{B R}\left(\tilde{\chi}_{1}^{ \pm} \rightarrow W^{\star} \tilde{\chi}_{1}^{0}\right)=100 \%$ and $m_{\tilde{\chi}_{1}^{ \pm}}=$ $m_{\tilde{\chi}_{1}^{0}}+5 \mathrm{GeV}$. 


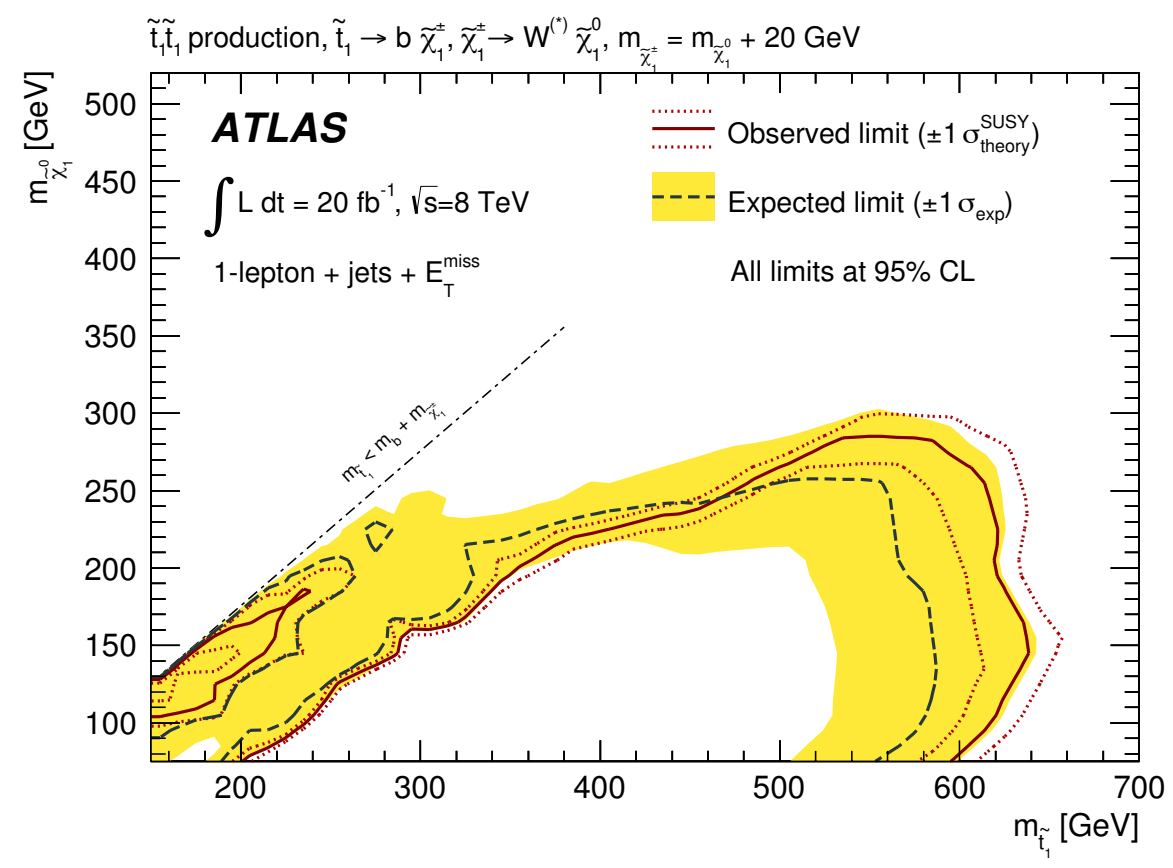

Figure 20. Expected (black dashed) and observed (red solid) 95\% CL excluded region in the plane of $m_{\tilde{\chi}_{1}^{0}}$ vs. $m_{\tilde{t}_{1}}$, assuming $\mathcal{B R}\left(\tilde{t}_{1} \rightarrow b \tilde{\chi}_{1}^{ \pm}\right)=100 \%, \mathcal{B R}\left(\tilde{\chi}_{1}^{ \pm} \rightarrow W^{\star} \tilde{\chi}_{1}^{0}\right)=100 \%$ and $m_{\tilde{\chi}_{1}^{ \pm}}=$ $m_{\tilde{\chi}_{1}^{0}}+20 \mathrm{GeV}$.

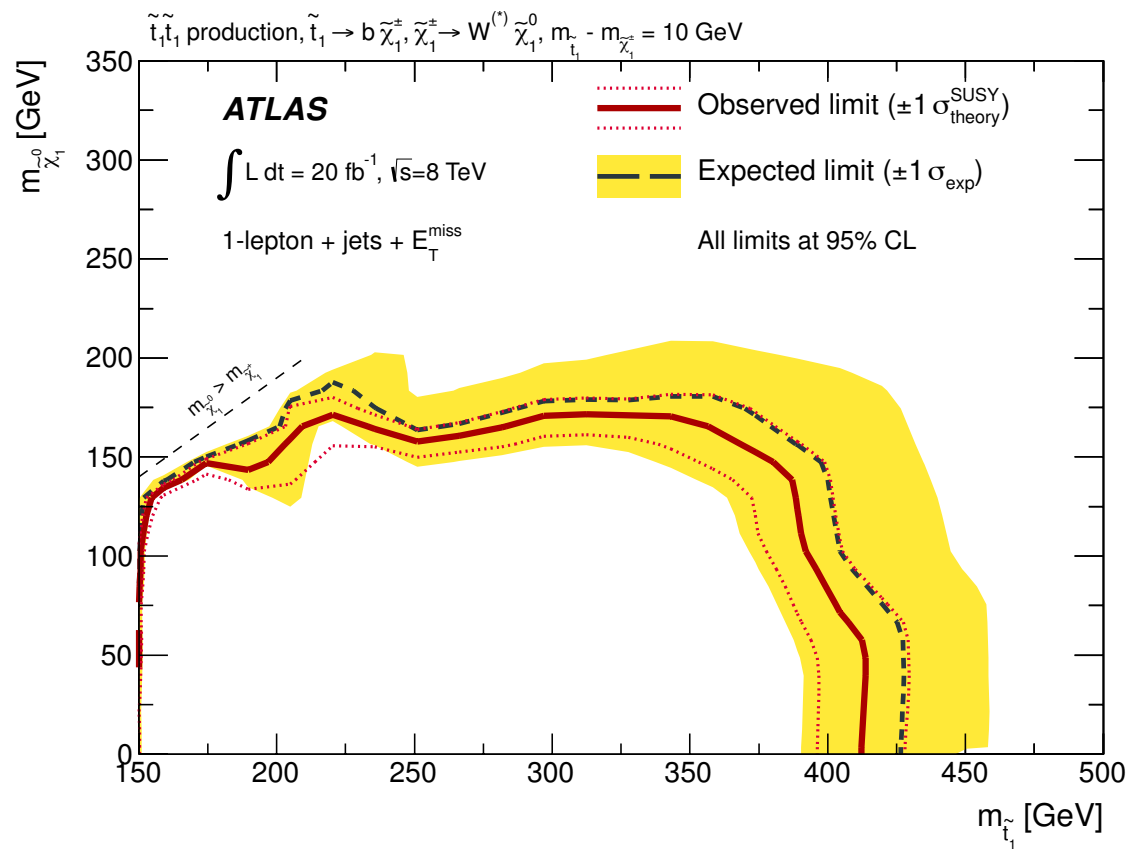

Figure 21. Expected (black dashed) and observed (red solid) 95\% CL excluded region in the plane of $m_{\tilde{\chi}_{1}^{0}}$ vs. $m_{\tilde{t}_{1}}$, assuming $\mathcal{B R}\left(\tilde{t}_{1} \rightarrow b \tilde{\chi}_{1}^{ \pm}\right)=100 \%, \mathcal{B R}\left(\tilde{\chi}_{1}^{ \pm} \rightarrow W^{(\star)} \tilde{\chi}_{1}^{0}\right)=100 \%$ and $m_{\tilde{\chi}_{1}^{ \pm}}=m_{\tilde{t}_{1}}-10 \mathrm{GeV}$. 


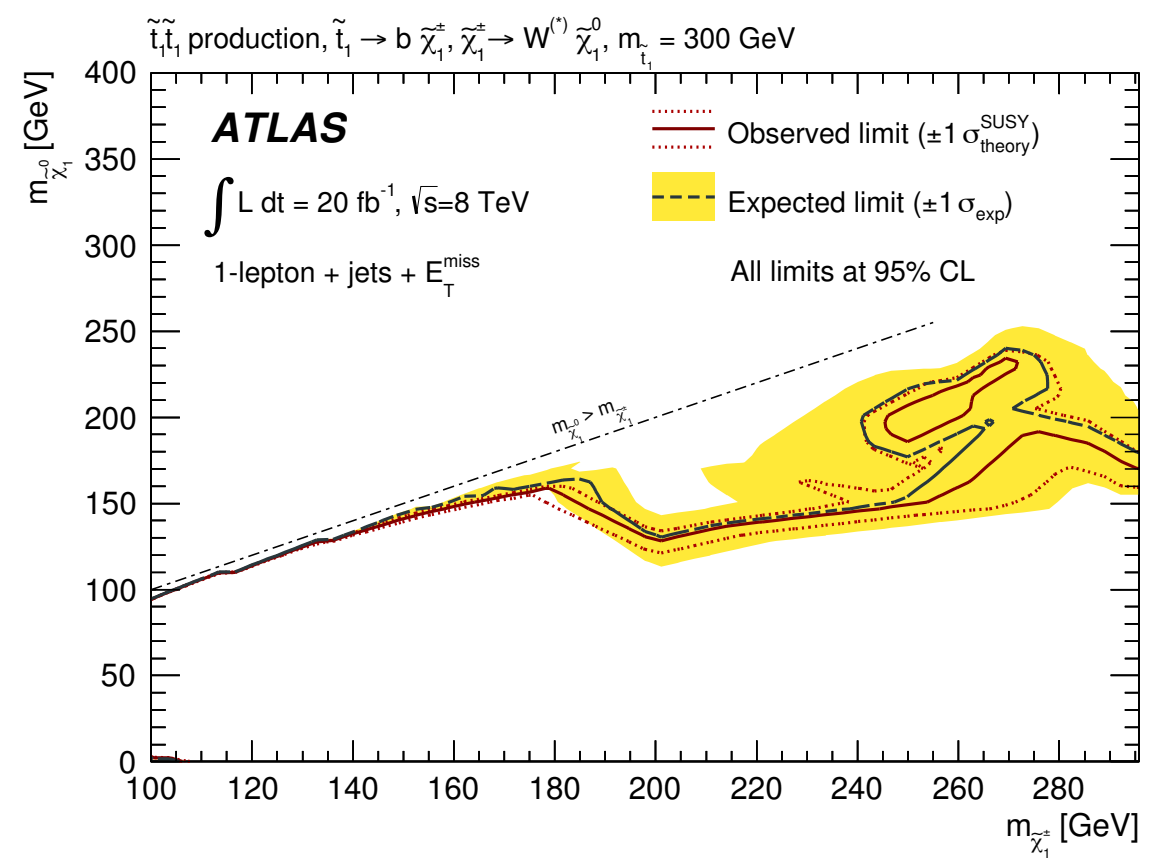

Figure 22. Expected (black dashed) and observed (red solid) 95\% CL excluded region in the plane of $m_{\tilde{\chi}_{1}^{0}}$ vs. $m_{\tilde{\chi}_{1}^{ \pm}}$, assuming $\mathcal{B R}\left(\tilde{t}_{1} \rightarrow b \tilde{\chi}_{1}^{ \pm}\right)=100 \%, \mathcal{B R}\left(\tilde{\chi}_{1}^{ \pm} \rightarrow W^{(\star)} \tilde{\chi}_{1}^{0}\right)=100 \%$ and $m_{\tilde{t}_{1}}=300 \mathrm{GeV}$.

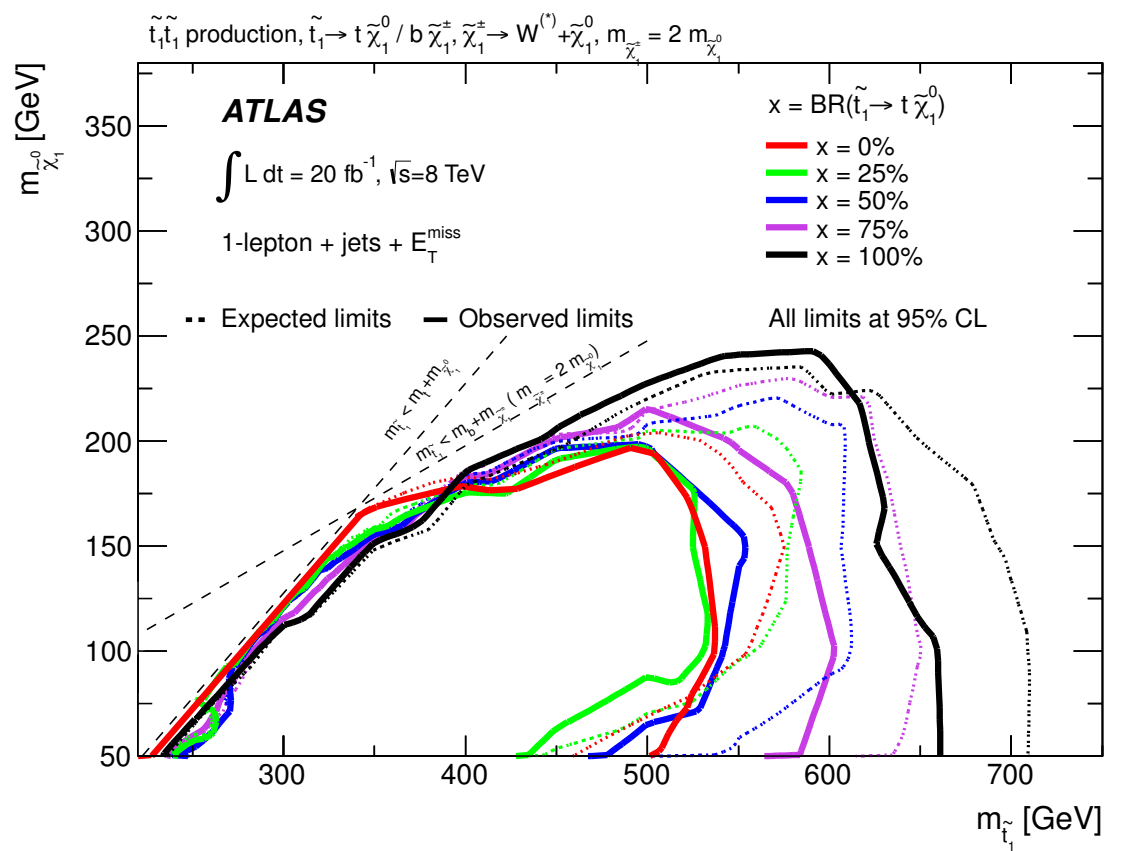

Figure 23. Expected (dashed) and observed (solid) 95\% CL excluded region in the plane of $m_{\tilde{\chi}_{1}^{0}}$ vs. $m_{\tilde{t}_{1}}$, assuming $x=\mathcal{B R}\left(\tilde{t}_{1} \rightarrow t \tilde{\chi}_{1}^{0}\right)=1-\mathcal{B R}\left(\tilde{t}_{1} \rightarrow b \tilde{\chi}_{1}^{ \pm}\right)$, and $x$ varying from $0 \%$ to $100 \%$. 

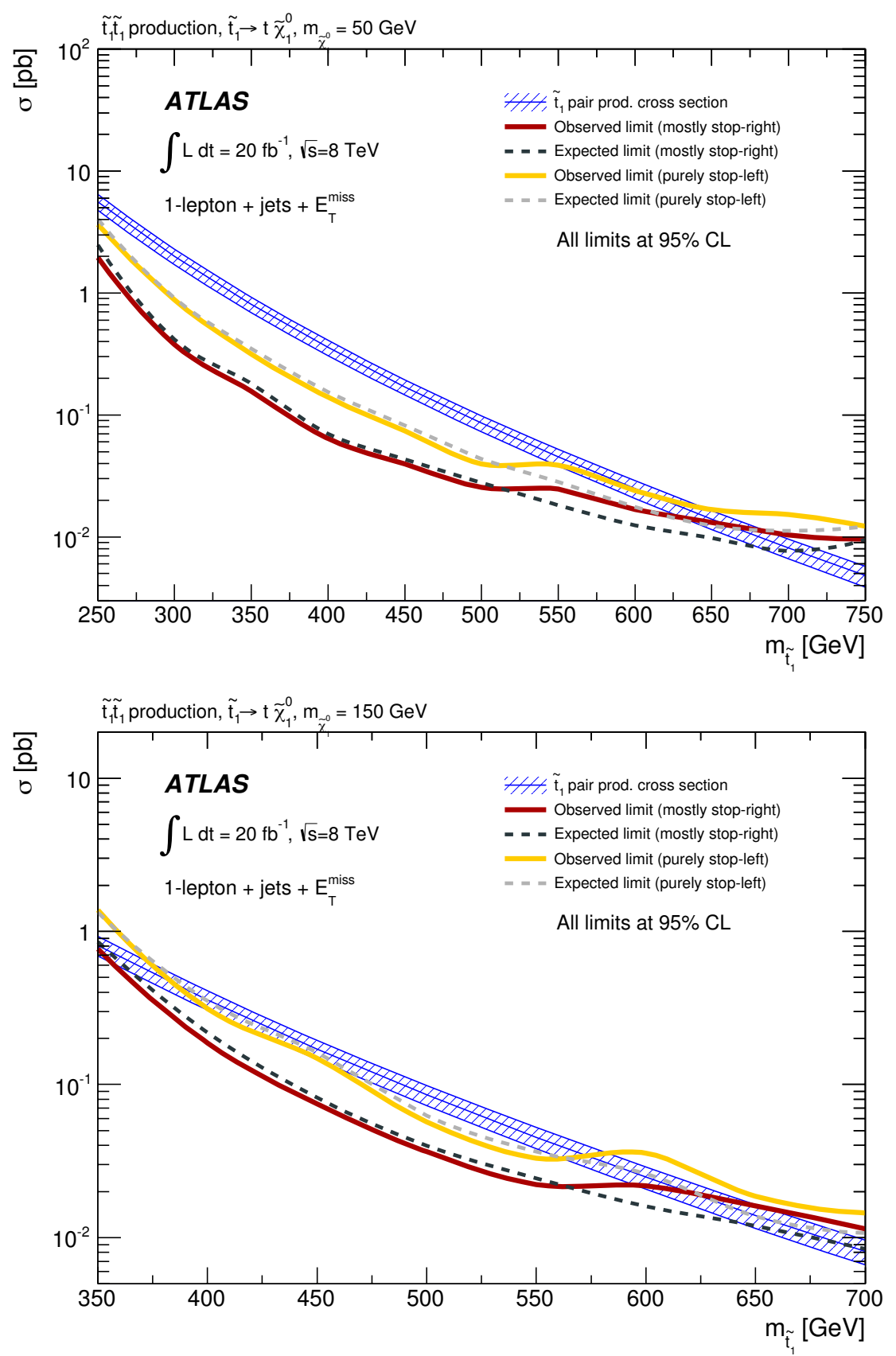

Figure 24. Expected and observed cross-section upper limits at 95\% CL in the $\tilde{t}_{1} \rightarrow t \tilde{\chi}_{1}^{0}$ decay mode with the LSP mass fixed to $50 \mathrm{GeV}$ (top) or $150 \mathrm{GeV}$ (bottom) for models with the $\tilde{t}_{1}$ being a pure stop-left $\left(\tilde{t}_{\mathrm{L}}\right)$ or mostly a stop-right $\left(\tilde{t}_{\mathrm{R}}\right)$. The upper and lower blue lines correspond to the nominal signal cross-section scaled up and down by the theoretical uncertainty. 

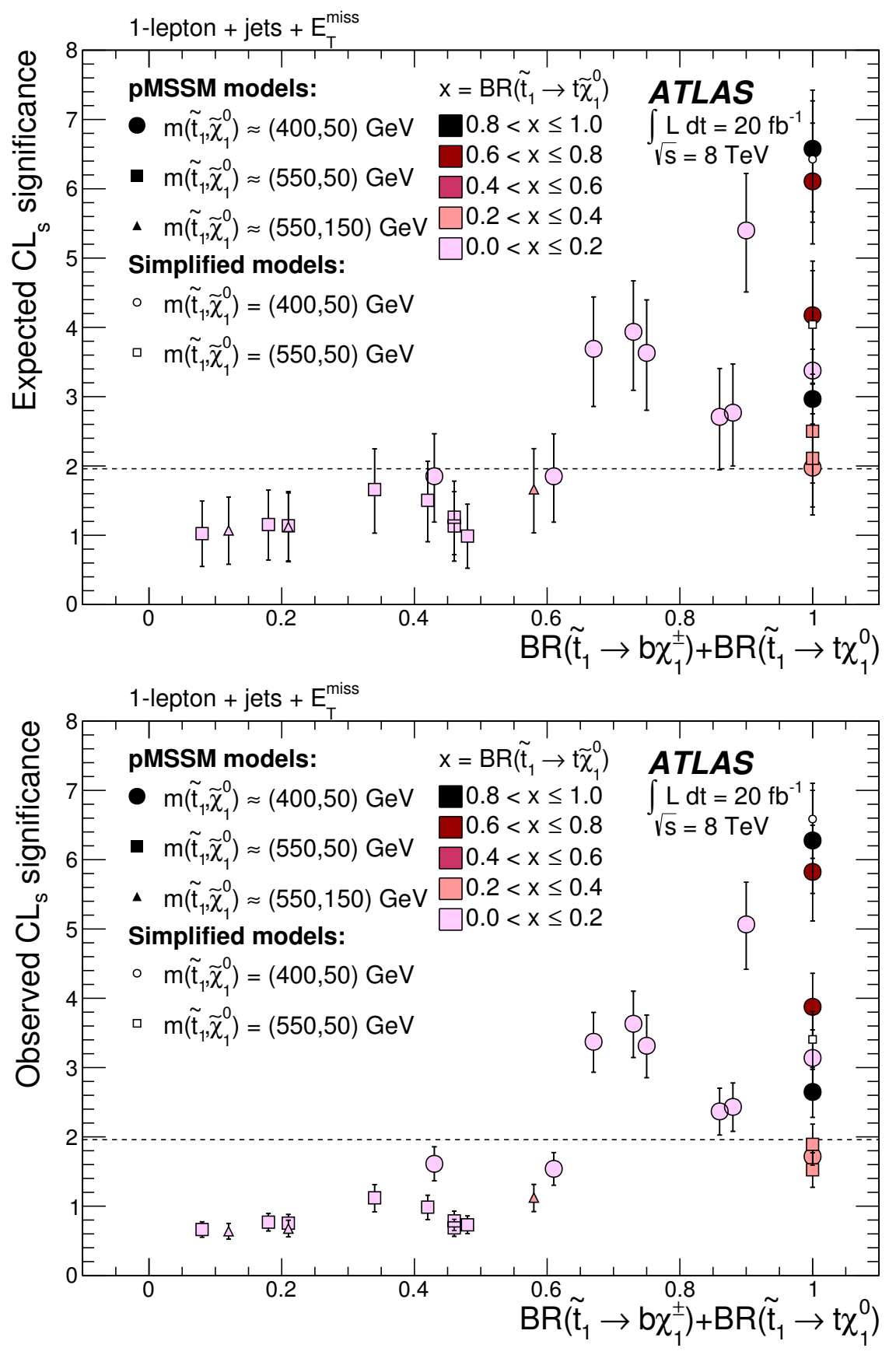

Figure 25. The expected (top) and observed (bottom) $\mathrm{CL}_{s}$ significance values for the $27 \mathrm{pMSSM}$ models described in section 5.2 and for two simplified models. Models above the dashed line, indicating the $\mathrm{CL}_{s}$ significance corresponding to $95 \% \mathrm{CL}$, are excluded. The results of the pMSSM models are displayed using filled markers where the marker symbol corresponds to the $m_{\tilde{t}_{1}}, m_{\tilde{\chi}_{1}^{0}}$ range and the colour represents the branching ratio for the $\tilde{t}_{1} \rightarrow t \tilde{\chi}_{1}^{0}$ decay, while the simplified models are shown using open markers. The uncertainty on the expected $\mathrm{CL}_{s}$ significance includes all sources except the theoretical cross-section uncertainty, while the uncertainty on the observed $\mathrm{CL}_{s}$ significance includes only the effect of scaling the nominal signal cross-section up and down by the theoretical cross-section uncertainty. 


\section{Acknowledgments}

We thank CERN for the very successful operation of the LHC, as well as the support staff from our institutions without whom ATLAS could not be operated efficiently.

We acknowledge the support of ANPCyT, Argentina; YerPhI, Armenia; ARC, Australia; BMWFW and FWF, Austria; ANAS, Azerbaijan; SSTC, Belarus; CNPq and FAPESP, Brazil; NSERC, NRC and CFI, Canada; CERN; CONICYT, Chile; CAS, MOST and NSFC, China; COLCIENCIAS, Colombia; MSMT CR, MPO CR and VSC CR, Czech Republic; DNRF, DNSRC and Lundbeck Foundation, Denmark; EPLANET, ERC and NSRF, European Union; IN2P3-CNRS, CEA-DSM/IRFU, France; GNSF, Georgia; BMBF, DFG, HGF, MPG and AvH Foundation, Germany; GSRT and NSRF, Greece; ISF, MINERVA, GIF, I-CORE and Benoziyo Center, Israel; INFN, Italy; MEXT and JSPS, Japan; CNRST, Morocco; FOM and NWO, Netherlands; BRF and RCN, Norway; MNiSW and NCN, Poland; GRICES and FCT, Portugal; MNE/IFA, Romania; MES of Russia and ROSATOM, Russian Federation; JINR; MSTD, Serbia; MSSR, Slovakia; ARRS and MIZŠ, Slovenia; DST/NRF, South Africa; MINECO, Spain; SRC and Wallenberg Foundation, Sweden; SER, SNSF and Cantons of Bern and Geneva, Switzerland; NSC, Taiwan; TAEK, Turkey; STFC, the Royal Society and Leverhulme Trust, United Kingdom; DOE and NSF, United States of America.

The crucial computing support from all WLCG partners is acknowledged gratefully, in particular from CERN and the ATLAS Tier-1 facilities at TRIUMF (Canada), NDGF (Denmark, Norway, Sweden), CC-IN2P3 (France), KIT/GridKA (Germany), INFN-CNAF (Italy), NL-T1 (Netherlands), PIC (Spain), ASGC (Taiwan), RAL (U.K.) and BNL (U.S.A.) and in the Tier-2 facilities worldwide.

\section{A Detailed description of the discriminating variables}

This section provides more detailed descriptions of the discriminating variables that are introduced in section 6 .

- Stransverse Mass, $m_{\mathrm{T} 2}$.

This variable targets decay topologies with two branches, referred to here as $a$ and $b$. In each branch, there are some particles with fully measured momenta and some particles with momenta that are not measured directly. The sum of the four vectors of the measured momenta in branch $i \in\{a, b\}$ are denoted $p_{i}=\left(E_{i}, \vec{p}_{\mathrm{T} i}, p_{z i}\right)$ and the sum of the four vectors of the unmeasured momenta are denoted $q_{i}=\left(F_{i}, \vec{q}_{\mathrm{T} i}, q_{z i}\right)$. With $m_{p_{i}}^{2}=$ $E_{i}^{2}-\vec{p}_{i}^{2}$ and $m_{q_{i}}^{2}=F_{i}^{2}-\vec{q}_{i}^{2}$, the $m_{\mathrm{T}}$ of the particles in branch $i$ is given in general by

$$
m_{\mathrm{T} i}^{2}=\left(\sqrt{p_{\mathrm{T} i}^{2}+m_{p_{i}}^{2}}+\sqrt{q_{\mathrm{T} i}^{2}+m_{q_{i}}^{2}}\right)^{2}-\left(\vec{p}_{\mathrm{T} i}+\vec{q}_{\mathrm{T} i}\right)^{2}
$$

which in the case that $m_{q_{i}}=m_{p_{i}}=0$ is the same as the one given in section 6.2. A generalisation of $m_{\mathrm{T}}, m_{\mathrm{T} 2}$, is defined as a minimisation over the allocation of $\vec{p}_{\mathrm{T}}^{\text {miss }}$ 
between $\vec{q}_{\mathrm{T} a}$ and $\vec{q}_{\mathrm{T} b}$ of the maximum of the corresponding $m_{\mathrm{Ta}}$ or $m_{\mathrm{Tb}}$ :

$$
m_{\mathrm{T} 2} \equiv \min _{\vec{q}_{\mathrm{T} a}+\vec{q}_{\mathrm{T} b}=\vec{p}_{\mathrm{T}}^{\operatorname{miss}}}\left\{\max \left(m_{\mathrm{T} a}, m_{\mathrm{T} b}\right)\right\},
$$

where one must make an assumption of $m_{q_{a}}$ and $m_{q_{b}}$ in the computation of $m_{T a}$ and $m_{T b}$. The result of the above minimisation is the minimum parent mass consistent with the observed kinematic distributions under the inputs $m_{q_{a}}$ and $m_{q_{b}}$. The variants of $m_{\mathrm{T} 2}$ described below only differ in the measured particles, (assumed) unmeasured particles, and choices for the input masses, $m_{q_{a}}$ and $m_{q_{b}}$.

- Asymmetric $m_{\mathrm{T} 2}, a m_{\mathrm{T} 2}$

- Measured particles: for branch $a$, this is one of the $b$-jets and for branch $b$ this is the second $b$-jet and the charged lepton. The $b$-jets are identified based on the highest $b$-tagging weights. Since there are two ways of assigning the $b$-tagged jets to branches $a$ and $b$, both $m_{\mathrm{T} 2}$ values are computed and the minimum kept for the final discriminant.

- Unmeasured particles: for branch $a$, this is a $W$ boson that decays leptonically, with the charged lepton unidentified as such. The unmeasured particle for branch $b$ is the neutrino associated with the measured charged lepton.

- Input masses: $m_{q_{a}}=m_{W}=80 \mathrm{GeV}$ and $m_{q_{b}}=m_{\nu}=0 \mathrm{GeV}$.

- In cases in which the lost lepton is an electron and the corresponding energy deposit enters the $E_{\mathrm{T}}^{\mathrm{miss}}$ calculation, for instance as a soft calorimeter cluster, $a m_{\mathrm{T} 2}$ can exceed the top mass boundary in $t \bar{t}$ events, but the variable remains powerful at discriminating signal from background.

- $\tau$-based $m_{\mathrm{T} 2}, m_{\mathrm{T} 2}^{\tau}$

- Measured particles: for branch $a$, this is the $\tau$-jet, identified as the highest- $p_{\mathrm{T}}$ jet excluding the selected two $b$-tagged jets. The measured particle for branch $b$ is the charged lepton.

- Unmeasured particles: for branch $a$, this includes the two neutrinos associated with the $\tau$ production and hadronic decay. The unmeasured particle for branch $b$ is the neutrino associated with the charged lepton.

- Input masses: $m_{q_{a}}=0 \mathrm{GeV}$ and $m_{q_{b}}=m_{\nu}=0 \mathrm{GeV}$.

\section{- Topness.}

The topness event value is defined as $\ln (\min \hat{S})$, where $\hat{S}$ is the minimum of the $\chi^{2}$-type function $S$ :

$$
\begin{aligned}
S\left(p_{W, x}, p_{W, y}, p_{W, z}, p_{\nu, z}\right)= & \frac{\left(m_{W}^{2}-\left(p_{\ell}+p_{\nu}\right)^{2}\right)^{2}}{a_{W}^{4}}+\frac{\left(m_{t}^{2}-\left(p_{b_{1}}+p_{\ell}+p_{\nu}\right)^{2}\right)^{2}}{a_{t}^{4}}+ \\
& +\frac{\left(m_{t}^{2}-\left(p_{b_{2}}+p_{W}\right)^{2}\right)^{2}}{a_{t}^{4}}+\frac{\left(4 m_{t}^{2}-(\Sigma p)^{2}\right)^{2}}{a_{\mathrm{CM}}^{4}} .
\end{aligned}
$$


The first three arguments of $S$ are the components of the non-reconstructed $W$ boson 3 -momentum $\left(p_{W, x}, p_{W, y}, p_{W, z}\right)$. This $W$ is assumed to decay leptonically, but the lepton is not reconstructed and is thus only noticeable in the missing transverse momentum. The variable $p_{\nu, z}$ is the longitudinal momentum of the neutrino from the other $W$ boson decay, for which the lepton was successfully reconstructed. These four numbers are varied to find the minimum of $S$.

The momenta appearing on the right-hand side of the equation above are either 4momenta of the reconstructed objects (one lepton, $p_{\ell}$, and two $b$-jets, $p_{b_{1}}$ and $p_{b_{2}}$ ) or 4 -momenta assigned by the minimisation procedure $\left(p_{W}\right.$ and $\left.p_{\nu}\right)$. To find all four components, the neutrinos and the $W$ boson without reconstructed decay products are assumed to be on-shell. Both combinations for $b_{1}$ and $b_{2}$ are evaluated during the minimisation; if only one $b$-tagged jet is present, it is used together with the leading or subleading jet (that means, a total of four possible jet assignments is evaluated in this case).

The minimisation is constrained such that the observed missing transverse momentum is attributed to the unobserved $W$ boson (decaying into a not-reconstructed lepton and a neutrino) and a neutrino from the other top decay branch.

The constants $a_{W}, a_{t}$ and $a_{\mathrm{CM}}$ are set to the values suggested by the authors of $S$ : $a_{W}=5 \mathrm{GeV}, a_{t}=15 \mathrm{GeV}, a_{\mathrm{CM}}=1 \mathrm{TeV}$.

- Hadronic top mass, $m_{\text {had-top }}$.

This reconstructed top mass is constructed as $m_{j_{1}, j_{2}, b_{i}}$ by minimising

$$
\chi^{2}=\frac{\left(m_{j_{1}, j_{2}, b_{i}}-m_{\mathrm{top}}\right)^{2}}{\sigma_{m_{j_{1}, j_{2}, b_{i}}}^{2}}+\frac{\left(m_{j_{1}, j_{2}}-m_{W}\right)^{2}}{\sigma_{m_{j_{1}, j_{2}}}^{2}},
$$

where $i=1$ or $2 ; b_{1}$ and $b_{2}$ are the two jets with the highest $b$-tagging weights; $j_{1}, j_{2}$ are the highest $p_{\mathrm{T}}$ jets from the selected jets in the event excluding $b_{1}$ and $b_{2}$ and

$$
\begin{aligned}
\sigma_{m_{j_{1}, j_{2}, b_{i}}}^{2} & =m_{j_{1}, j_{2}, b_{i}}^{2}\left(r_{j_{1}}^{2}+r_{j_{2}}^{2}+r_{b_{i}}^{2}\right) \\
\sigma_{m_{j_{1}, j_{2}}}^{2} & =m_{j_{1}, j_{2}}^{2}\left(r_{j_{1}}^{2}+r_{j_{2}}^{2}\right),
\end{aligned}
$$

where $r_{i}$ is the fractional jet energy uncertainty of the $p_{\mathrm{T}}$ for jet $i$ determined by dedicated studies [105, 129].

- $\tau$-veto.

For the construction of the $\tau$-veto, the reconstructed $\tau_{\text {had }}$ candidates are subject to further selection requirements. Candidates are required to have either one associated track (classified as one-prong $\tau$ decay), or two to three tracks (classified as three-prong $\tau$ decay, where one track can be missed). The $\tau_{\text {had }}$ charge for candidates with one or three tracks is required to be \pm 1 and to be opposite to the charge of the selected electron or muon in the event. For candidates with two tracks, the sign of the $\tau_{\text {had }}$ charge is required to be opposite to that of the selected lepton only if the $\tau_{\text {had }}$ charge is \pm 2 . Finally, 
three different BDT requirements are imposed on the candidates to define three $\tau$-veto working points: loose, tight, and extra-tight. In simulated $t \bar{t}$ events with one $W \rightarrow \ell \nu$ decay, signal- and background-like events are defined by requiring the other $W$ boson to either decay into quarks (signal) or into a $\tau_{\text {had }}$ (background). In these samples, the loose (tight) $\tau$-veto retains $99 \%$ (97\%) of signal events, while for background events $81 \%$ $(69 \%)$ with a one-prong and $75 \%(63 \%)$ with a three-prong $\tau_{\text {had }}$ decay survive the veto.

- Track-veto.

Tracks are required to satisfy the following criteria: $p_{\mathrm{T}}>10 \mathrm{GeV}$ and $|\eta|<2.5$, transverse and longitudinal impact parameters $\left|d_{0}\right|<1 \mathrm{~mm}$ and $\left|z_{0}\right|<2 \mathrm{~mm}$. The track isolation requires that there are no additional tracks associated with the primary vertex with $p_{\mathrm{T}}>3 \mathrm{GeV}$ in a cone of $\Delta R=0.4$ around the track. Events with at least one isolated track of opposite charge compared to that of the selected electron or muon in the event are rejected by the track-veto.

- $H_{\mathrm{T}, \mathrm{sig}}^{\mathrm{miss}}$ is an object-based missing transverse momentum, divided by the per-event resolution of the jets. It is defined by

$$
H_{\mathrm{T}, \mathrm{sig}}^{\mathrm{miss}}=\frac{\left|\vec{H}_{\mathrm{T}}^{\mathrm{miss}}\right|-M}{\sigma_{\left|\vec{H}_{\mathrm{T}}^{\mathrm{miss}}\right|}},
$$

where $\vec{H}_{\mathrm{T}}^{\mathrm{miss}}$ is the negative sum of the jets and lepton vectors. The denominator is computed from the per-event jet energy uncertainties, while the lepton is assumed to be well-measured. The parameter $M$ is chosen to be a characteristic 'scale' of the background [145], and is fixed at $100 \mathrm{GeV}$ in this analysis based on optimisation studies.

\section{B Background fit results}

This section contains the background fit results for all analyses. The model-dependent selection is used. The CR and SR bins (cut-and-count and one-dimensional shape-fits) or the full set of bins (two-dimensional shape-fits) are included in the likelihood. However, a potential signal contribution is neglected everywhere (the signal strength is fixed to zero). All background uncertainties are taken into account. This fit configuration is different from the background-only fit, which is used for the validation results in section 8 , in that it includes more bins to constrain the likelihood. The results of the cut-and-count analyses are given in table 12, and the results for the shape-fits are shown in tables 13-20. 


\begin{tabular}{|c|c|c|c|c|c|c|c|c|c|}
\hline \multirow{2}{*}{ Region } & \multirow{2}{*}{ Obs. } & \multicolumn{8}{|c|}{ Fitted (estimated) background } \\
\hline & & \multicolumn{2}{|c|}{ Total } & \multicolumn{2}{|c|}{$t \bar{t}$} & \multicolumn{2}{|c|}{$W+$ jets } & \multicolumn{2}{|l|}{ Other } \\
\hline TCR & 159 & $159 \pm 12$ & $(155)$ & $124 \pm 14$ & $(108)$ & $19 \pm 4$ & $(32)$ & $15.8 \pm 2.6$ & $(16)$ \\
\hline WCR & 161 & $161 \pm 13$ & $(232)$ & $36 \pm 9$ & $(31)$ & $109 \pm 17$ & $(185)$ & $16 \pm 5$ & (15) \\
\hline TVR & 16 & $26 \pm 6$ & $(26)$ & $20 \pm 5$ & (17) & $3.4 \pm 1.0$ & $(6)$ & $3.1 \pm 0.7$ & (3) \\
\hline WVR & 25 & $24 \pm 7$ & $(34)$ & $6.7 \pm 2.5$ & (6) & $15 \pm 5$ & $(26)$ & $2.0 \pm 1.0$ & $(2)$ \\
\hline SR & 12 & $12.7 \pm 1.8$ & (14) & $6.3 \pm 1.5$ & (6) & $2.0 \pm 0.5$ & (3) & $4.4 \pm 0.9$ & (4) \\
\hline tN_high & & & & & & & & & \\
\hline TCR & 359 & $359 \pm 19$ & $(361)$ & $287 \pm 22$ & $(274)$ & $39 \pm 7$ & $(55)$ & $32 \pm 6$ & $(32)$ \\
\hline WCR & 483 & $483 \pm 22$ & $(615)$ & $100 \pm 24$ & $(95)$ & $340 \pm 40$ & $(475)$ & $44 \pm 12$ & $(42)$ \\
\hline TVR & 59 & $79 \pm 18$ & $(79)$ & $64 \pm 15$ & $(61)$ & $6.8 \pm 2.1$ & $(10)$ & $8.3 \pm 1.2$ & $(8)$ \\
\hline WVR & 74 & $84 \pm 24$ & (104) & $22 \pm 7$ & (21) & $52 \pm 16$ & (73) & $9.7 \pm 2.1$ & (9) \\
\hline SR & 5 & $5.0 \pm 0.9$ & $(5)$ & $2.0 \pm 0.6$ & $(2)$ & $0.87 \pm 0.26$ & (1) & $2.2 \pm 0.4$ & $(2)$ \\
\hline tN_boost & & & & & & & & & \\
\hline TCR & 117 & $118 \pm 11$ & (111) & $96 \pm 11$ & (80) & $10.8 \pm 2.2$ & $(20)$ & $11.2 \pm 1.9$ & (11) \\
\hline WCR & 210 & $210 \pm 14$ & (332) & $35 \pm 10$ & (29) & $149 \pm 20$ & (278) & $26 \pm 7$ & (24) \\
\hline TVR & 20 & $23 \pm 5$ & $(21)$ & $19 \pm 5$ & (15) & $1.9 \pm 0.4$ & $(3)$ & $2.5 \pm 0.6$ & $(2)$ \\
\hline WVR & 29 & $35 \pm 7$ & (51) & $7.7 \pm 2.3$ & $(6)$ & $21 \pm 5$ & (38) & $6.3 \pm 1.2$ & (6) \\
\hline SR & 5 & $3.5 \pm 0.7$ & (3) & $1.2 \pm 0.4$ & $(0.9)$ & $0.31 \pm 0.14$ & $(0.5)$ & $2.1 \pm 0.4$ & $(2)$ \\
\hline bCc_diag & & & & & & & & & \\
\hline TCR & 1650 & $1650 \pm 40$ & (1689) & $1240 \pm 60$ & (1246) & $244 \pm 34$ & (278) & $164 \pm 24$ & (162) \\
\hline WCR & 2162 & $2160 \pm 50$ & (2361) & $350 \pm 60$ & $(334)$ & $1670 \pm 90$ & (1890) & $141 \pm 25$ & (127) \\
\hline TVR & 925 & $890 \pm 100$ & $(876)$ & $770 \pm 90$ & $(752)$ & $46 \pm 7$ & $(51)$ & $73 \pm 7$ & (71) \\
\hline WVR & 693 & $740 \pm 160$ & (781) & $163 \pm 35$ & (155) & $520 \pm 130$ & (567) & $60 \pm 6$ & (52) \\
\hline SR & 493 & $489 \pm 21$ & (501) & $158 \pm 34$ & (145) & $250 \pm 27$ & (276) & $82 \pm 17$ & (74) \\
\hline bCd_high1 & & & & & & & & & \\
\hline TCR & 218 & $220 \pm 15$ & (228) & $171 \pm 17$ & $(172)$ & $22 \pm 5$ & (29) & $28 \pm 5$ & $(27)$ \\
\hline WCR & 757 & $758 \pm 28$ & (1002) & $87 \pm 25$ & $(86)$ & $610 \pm 40$ & (860) & $57 \pm 16$ & (55) \\
\hline TVR & 42 & $53 \pm 13$ & $(53)$ & $43 \pm 11$ & (41) & $4.5 \pm 1.3$ & $(6)$ & $6.3 \pm 1.2$ & (6) \\
\hline WVR & 166 & $148 \pm 35$ & (191) & $23 \pm 8$ & (23) & $111 \pm 29$ & (153) & $14.4 \pm 3.4$ & (14) \\
\hline SR & 16 & $11.8 \pm 1.4$ & $(12)$ & $5.6 \pm 1.0$ & $(5)$ & $2.1 \pm 0.6$ & $(3)$ & $4.2 \pm 1.0$ & $(4)$ \\
\hline bCd_high2 & & & & & & & & & \\
\hline TCR & 129 & $129 \pm 11$ & (154) & $92 \pm 13$ & (110) & $16 \pm 4$ & $(23)$ & $22 \pm 4$ & $(22)$ \\
\hline WCR & 654 & $654 \pm 26$ & (911) & $56 \pm 19$ & $(67)$ & $550 \pm 40$ & (792) & $53 \pm 16$ & $(52)$ \\
\hline TVR & 28 & $32 \pm 6$ & $(38)$ & $23 \pm 5$ & (28) & $3.6 \pm 0.9$ & $(5)$ & $5.7 \pm 1.3$ & (6) \\
\hline WVR & 157 & $135 \pm 29$ & (185) & $16 \pm 6$ & (19) & $106 \pm 25$ & (152) & $13.7 \pm 2.9$ & (13) \\
\hline SR & 5 & $4.5 \pm 0.8$ & $(5)$ & $1.8 \pm 0.4$ & $(2)$ & $0.73 \pm 0.32$ & $(1)$ & $2.0 \pm 0.5$ & $(2)$ \\
\hline tNbC_mix & & & & & & & & & \\
\hline TCR & 177 & $178 \pm 13$ & (168) & $139 \pm 15$ & (125) & $20 \pm 4$ & $(25)$ & $18.5 \pm 3.5$ & (18) \\
\hline WCR & 387 & $387 \pm 20$ & (446) & $80 \pm 21$ & $(71)$ & $281 \pm 31$ & (349) & $27 \pm 7$ & $(25)$ \\
\hline TVR & 64 & $77 \pm 19$ & $(70)$ & $63 \pm 16$ & (54) & $7.4 \pm 1.8$ & (9) & $7.4 \pm 1.8$ & (7) \\
\hline WVR & 118 & $130 \pm 40$ & (139) & $33 \pm 13$ & (29) & $84 \pm 28$ & (101) & $9.4 \pm 2.0$ & $(8)$ \\
\hline SR & 10 & $7.6 \pm 1.0$ & $(7)$ & $3.0 \pm 0.6$ & (3) & $1.32 \pm 0.35$ & $(2)$ & $3.2 \pm 0.7$ & (3) \\
\hline
\end{tabular}

Table 12. Results of the all-bins background fit for the cut-and-count analyses. The numbers in parenthesis are the pre-fit background estimates using the most accurate theoretical cross-sections available (cf. section 5). 


\begin{tabular}{|c|c|c|c|c|c|c|}
\hline \multirow{2}{*}{\multicolumn{2}{|c|}{ tN_diag }} & \multirow{2}{*}{ Obs. } & \multicolumn{4}{|c|}{ Fitted (estimated) background } \\
\hline & & & Total & $t \bar{t}$ & $W+$ jets & Other \\
\hline \multirow{5}{*}{ 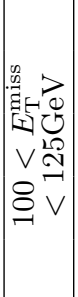 } & $60<m_{\mathrm{T}}<90 \mathrm{GeV}+b$-veto & 1647 & $1650 \pm 40(1896)$ & $550 \pm 130$ & $990 \pm 150(1159)$ & $108 \pm 24$ \\
\hline & $60<m_{\mathrm{T}}<90 \mathrm{GeV}$ & 3462 & $3450 \pm 60(3402)$ & $3090 \pm 90(3005)$ & $180 \pm 40 \quad(212)$ & $180 \pm 50(184)$ \\
\hline & $90<m_{\mathrm{T}}<120 \mathrm{GeV}$ & 1712 & $1720 \pm 40(1693)$ & $1540 \pm 50(1487)$ & $96 \pm 23$ & $84 \pm 27 \quad(89)$ \\
\hline & $120<m_{\mathrm{T}}<140 \mathrm{GeV}$ & 313 & $293 \pm 12$ & $263 \pm 12$ & $16 \pm 4$ & $14 \pm 4$ \\
\hline & $m_{\mathrm{T}}>140 \mathrm{GeV}$ & 201 & $222 \pm 11$ & $202 \pm 12$ & $7 \pm 4$ & $12.8 \pm 3.1(13)$ \\
\hline \multirow{5}{*}{ 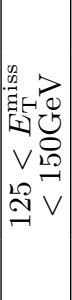 } & $60<m_{\mathrm{T}}<90 \mathrm{GeV}+b$-veto & 1081 & $1081 \pm 33(1284)$ & $390 \pm 80$ & $620 \pm 90 \quad(817)$ & $71 \pm 15 \quad(67)$ \\
\hline & $60<m_{\mathrm{T}}<90 \mathrm{GeV}$ & 2018 & $2020 \pm 40(1984)$ & $1800 \pm 60(1718)$ & $111 \pm 25$ & $114 \pm 34$ \\
\hline & $90<m_{\mathrm{T}}<120 \mathrm{GeV}$ & 768 & $764 \pm 24$ & $690 \pm 26$ & $32 \pm 10$ & $42 \pm 13$ \\
\hline & $120<m_{\mathrm{T}}<140 \mathrm{GeV}$ & 117 & $130 \pm 8 \quad(134)$ & $117 \pm 8$ & $6.0 \pm 2.5$ & $6.7 \pm 1.9$ \\
\hline & $m_{\mathrm{T}}>140 \mathrm{GeV}$ & 163 & $151 \pm 7 \quad(149)$ & $136 \pm 8 \quad(131)$ & $6.2 \pm 2.6$ & $9.3 \pm 2.1 \quad(9)$ \\
\hline \multirow{5}{*}{ 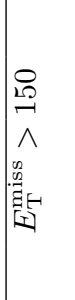 } & $60<m_{\mathrm{T}}<90 \mathrm{GeV}+b$-veto & 1742 & $1740 \pm 40(2172)$ & $540 \pm 110$ & $1080 \pm 130(1508)$ & $127 \pm 28$ \\
\hline & $60<m_{\mathrm{T}}<90 \mathrm{GeV}$ & 2543 & $2540 \pm 50(2554)$ & $2170 \pm 80(2099)$ & $200 \pm 40 \quad(271)$ & $170 \pm 50(182)$ \\
\hline & $90<m_{\mathrm{T}}<120 \mathrm{GeV}$ & 647 & $651 \pm 24$ & $565 \pm 25$ & $41 \pm 9$ & $45 \pm 13$ \\
\hline & $120<m_{\mathrm{T}}<140 \mathrm{GeV}$ & 101 & $95 \pm 6 \quad(97)$ & $82 \pm 6 \quad(82)$ & $4.5 \pm 1.4$ & $8.3 \pm 2.0$ \\
\hline & $m_{\mathrm{T}}>140 \mathrm{GeV}$ & 217 & $223 \pm 12$ & $192 \pm 13$ & $8.8 \pm 2.8$ & $23 \pm 5$ \\
\hline
\end{tabular}

Table 13. Results of the all-bins background fit for the $t N_{-}$diag analysis. The numbers in parenthesis are the pre-fit background estimates using the most accurate theoretical cross-sections available (cf. section 5).

\begin{tabular}{|l|c|cc|cc|cc|cc|}
\hline \multirow{2}{*}{ bCa_low } & \multirow{2}{*}{ Obs. } & \multicolumn{6}{|c|}{ Fitted (estimated) background } \\
\cline { 3 - 9 } & & \multicolumn{2}{|c|}{ Total } & \multicolumn{2}{|c|}{$t \bar{t}$} & \multicolumn{2}{c|}{$W+$ jets } & \multicolumn{2}{c|}{ Other } \\
\hline TCR & 336 & $338 \pm 18$ & $(409)$ & $192 \pm 35(250)$ & $74 \pm 21$ & $(87)$ & $72 \pm 18$ & $(72)$ \\
WCR & 1149 & $1149 \pm 34(1333)$ & $62 \pm 17$ & $(78)$ & $940 \pm 50$ & $(1115)$ & $145_{-29}^{+34}$ & $(140)$ \\
VR & 74 & $75 \pm 12$ & $(90)$ & $44 \pm 10$ & $(56)$ & $12.3 \pm 2.1$ & $(14)$ & $19_{-7}^{+7}$ & $(19)$ \\
$6<p_{\mathrm{T}}^{\ell}<17 \mathrm{GeV}$ & 6 & $4.1 \pm 1.1$ & $(4)$ & $1.6 \pm 0.6$ & $(2)$ & $0.6 \pm 0.4$ & $(0.7)$ & $1.8_{-0.7}^{+0.8}$ & $(2)$ \\
$17<p_{\mathrm{T}}^{\ell}<28 \mathrm{GeV}$ & 6 & $3.7 \pm 0.9$ & $(4)$ & $1.7 \pm 0.7$ & $(2)$ & $0.76 \pm 0.24$ & $(0.9)$ & $1.2_{-0.4}^{+0.5}$ & $(1)$ \\
$28<p_{\mathrm{T}}^{\ell}<39 \mathrm{GeV}$ & 4 & $4.0 \pm 0.9$ & $(5)$ & $1.7 \pm 0.6$ & $(2)$ & $0.77 \pm 0.26$ & $(0.9)$ & $1.6_{-0.6}^{+0.6}$ & $(2)$ \\
$39<p_{\mathrm{T}}^{\ell}<50 \mathrm{GeV}$ & 3 & $2.8 \pm 0.7$ & $(3)$ & $1.2 \pm 0.7$ & $(1)$ & $0.82 \pm 0.34$ & $(0.9)$ & $0.80 \pm 0.29$ & $(0.8)$ \\
\hline
\end{tabular}

Table 14. Results of the all-bins background fit for the bCa_low analysis. The numbers in parenthesis are the pre-fit background estimates using the most accurate theoretical cross-sections available (cf. section 5). 


\begin{tabular}{|l|c|cc|cc|ccc|cc|}
\hline \multirow{2}{*}{ bCa_med } & \multirow{2}{*}{ Obs. } & \multicolumn{7}{|c|}{ Fitted (estimated) background } \\
\cline { 3 - 10 } & & \multicolumn{2}{|c|}{ Total } & \multicolumn{2}{|c|}{$t \bar{t}$} & \multicolumn{2}{c|}{$W+$ jets } & \multicolumn{2}{c|}{ Other } \\
\hline TCR & 136 & $136 \pm 11$ & $(157)$ & $98 \pm 16$ & $(111)$ & $15 \pm 5$ & $(23)$ & $23_{-9}^{+10}$ & $(23)$ \\
$\mathrm{WCR}$ & 189 & $189 \pm 14$ & $(259)$ & $26 \pm 7$ & $(29)$ & $121 \pm 19$ & $(188)$ & $42_{-11}^{+11}$ & $(42)$ \\
$\mathrm{VR}$ & 29 & $28 \pm 5$ & $(31)$ & $19 \pm 4$ & $(22)$ & $1.7 \pm 0.5$ & $(3)$ & $6.7_{-2.2}^{+2.4}$ & $(7)$ \\
$6<p_{\mathrm{T}}^{\ell}<17 \mathrm{GeV}$ & 9 & $9.2 \pm 2.0$ & $(10)$ & $5.3 \pm 1.3$ & $(6)$ & $0.61 \pm 0.21$ & $(0.9)$ & $3.3 \pm 1.5$ & $(3)$ \\
$17<p_{\mathrm{T}}^{\ell}<28 \mathrm{GeV}$ & 15 & $10.9 \pm 1.7$ & $(12)$ & $7.1 \pm 1.5$ & $(8)$ & $1.00 \pm 0.32$ & $(1)$ & $2.8_{-1.0}^{+1.1}$ & $(3)$ \\
$28<p_{\mathrm{T}}^{\ell}<39 \mathrm{GeV}$ & 6 & $10.9 \pm 1.7$ & $(12)$ & $7.1 \pm 1.4$ & $(8)$ & $0.87 \pm 0.31$ & $(1)$ & $2.9 \pm 1.0$ & $(3)$ \\
$39<p_{\mathrm{T}}^{\ell}<50 \mathrm{GeV}$ & 9 & $8.2 \pm 1.4$ & $(9)$ & $5.7 \pm 1.3$ & $(6)$ & $0.90 \pm 0.35$ & $(1)$ & $1.6_{-0.5}^{+0.5}$ & $(2)$ \\
\hline
\end{tabular}

Table 15. Results of the all-bins background fit for the bCa med analysis. The numbers in parenthesis are the pre-fit background estimates using the most accurate theoretical cross-sections available (cf. section 5).

\begin{tabular}{|l|c|cc|c|ccc|cc|}
\hline \multirow{2}{*}{ bCb_med1 } & \multirow{2}{*}{ Obs. } & \multicolumn{7}{|c|}{ Fitted (estimated) background } \\
\cline { 3 - 9 } & & \multicolumn{2}{|c|}{ Total } & \multicolumn{2}{|c|}{$t \bar{t}$} & \multicolumn{2}{c|}{$W+$ jets } & \multicolumn{2}{c|}{ Other } \\
\hline TCR & 390 & $397 \pm 20$ & $(405)$ & $287 \pm 28$ & $(289)$ & $45 \pm 12$ & $(50)$ & $65_{-10}^{+11}$ & $(65)$ \\
$\mathrm{WCR}$ & $1372413720 \pm 130(14480)$ & $880 \pm 200$ & $(868)$ & $11740 \pm 330(12507)$ & $1100_{-150}^{+190}$ & $(1104)$ \\
$\mathrm{VR}$ & 76 & $82 \pm 11$ & $(79)$ & $50 \pm 10$ & $(47)$ & $22.0 \pm 3.3$ & $(23)$ & $10 \pm 4$ & $(9)$ \\
$83.3<a m_{\mathrm{T} 2}<166.7 \mathrm{GeV}$ & 111 & $97 \pm 11$ & $(95)$ & $73 \pm 9$ & $(70)$ & $3.4 \pm 0.7$ & $(4)$ & $21_{-5}^{+8}$ & $(21)$ \\
$166.7<a m_{\mathrm{T} 2}<250.0 \mathrm{GeV}$ & 42 & $35 \pm 4$ & $(33)$ & $20 \pm 4$ & $(18)$ & $7.8 \pm 1.2$ & $(8)$ & $6.8_{-1.2}^{+2.3}$ & $(7)$ \\
$250.0<a m_{\mathrm{T} 2}<333.3 \mathrm{GeV}$ & 2 & $3.5 \pm 0.8$ & $(4)$ & $1.2 \pm 0.4$ & $(1)$ & $1.5 \pm 0.4$ & $(2)$ & $0.82_{-0.22}^{+0.28}$ & $(0.8)$ \\
$333.3<a m_{\mathrm{T} 2}<416.7 \mathrm{GeV}$ & 0 & $0.58 \pm 0.28$ & $(0.6)$ & $0.21_{-0.21}^{+0.22}(0.2)$ & $0.31 \pm 0.12$ & $(0.3)$ & $0.064_{-0.028}^{+0.028}$ & $(0.1)$ \\
$416.7<a m_{\mathrm{T} 2}<500.0 \mathrm{GeV}$ & 0 & $0.15 \pm 0.10$ & $(0.1)$ & $0.025_{-0.025}^{+0.050}(0.0)$ & $0.06 \pm 0.06$ & $(0.1)$ & $0.06 \pm 0.04$ & $(0.1)$ \\
\hline
\end{tabular}

Table 16. Results of the all-bins background fit for the bCb_med1 analysis. The numbers in parenthesis are the pre-fit background estimates using the most accurate theoretical cross-sections available (cf. section 5).

\begin{tabular}{|l|c|c|cc|ccc|cc|}
\hline \multirow{2}{*}{ bCb_high } & \multirow{2}{*}{ Obs. } & \multicolumn{7}{|c|}{ Fitted (estimated) background } \\
\cline { 3 - 10 } & & \multicolumn{2}{|c|}{ Total } & \multicolumn{2}{|c|}{$t \bar{t}$} & \multicolumn{2}{|c|}{$W+$ jets } & \multicolumn{2}{|c|}{ Other } \\
\hline TCR & 1111 & $1108 \pm 34(1131)$ & $880 \pm 50$ & $(881)$ & $62 \pm 17$ & $(82)$ & $165_{-28}^{+30}$ & $(169)$ \\
WCR & 4089 & $4090 \pm 60$ & $(4968)$ & $530 \pm 90$ & $(535)$ & $3140 \pm 130$ & $(4010)$ & $420_{-50}^{+60}$ & $(423)$ \\
VR & 12 & $13.9 \pm 3.0$ & $(16)$ & $9.3 \pm 2.7$ & $(10)$ & $2.9 \pm 0.6$ & $(4)$ & $1.7_{-0.5}^{+1.0}$ & $(2)$ \\
$83.3<a m_{\mathrm{T} 2}<166.7 \mathrm{GeV}$ & 11 & $12.9 \pm 2.0$ & $(14)$ & $11.4 \pm 1.9$ & $(12)$ & $0.034 \pm 0.028$ & $(0.0)$ & $1.5 \pm 0.6$ & $(2)$ \\
$166.7<a m_{\mathrm{T} 2}<250.0 \mathrm{GeV}$ & 6 & $5.8 \pm 1.2$ & $(6)$ & $4.0 \pm 1.1$ & $(4)$ & $0.78 \pm 0.26$ & $(1)$ & $1.07 \pm 0.31$ & $(1)$ \\
$250.0<a m_{\mathrm{T} 2}<333.3 \mathrm{GeV}$ & 4 & $5.1 \pm 0.8$ & $(6)$ & $2.0 \pm 0.6$ & $(2)$ & $1.23 \pm 0.32$ & $(2)$ & $1.9 \pm 0.5$ & $(2)$ \\
$333.3<a m_{\mathrm{T} 2}<416.7 \mathrm{GeV}$ & 1 & $1.7 \pm 0.6$ & $(2)$ & $0.47 \pm 0.28(0.6)$ & $0.29 \pm 0.15$ & $(0.4)$ & $0.9_{-0.4}^{+0.4}$ & $(0.9)$ \\
$416.7<a m_{\mathrm{T} 2}<500.0 \mathrm{GeV}$ & 0 & $0.49 \pm 0.23$ & $(0.6)$ & $0.08_{-0.08}^{+0.10}$ & $(0.1)$ & $0.17 \pm 0.08$ & $(0.2)$ & $0.24_{-0.17}^{+0.18}$ & $(0.2)$ \\
\hline
\end{tabular}

Table 17. Results of the all-bins background fit for the bCb_high analysis. The numbers in parenthesis are the pre-fit background estimates using the most accurate theoretical cross-sections available (cf. section 5). 


\begin{tabular}{|c|c|c|c|c|c|c|}
\hline \multirow{2}{*}{\multicolumn{2}{|c|}{$\mathrm{bCb} \_$med2 }} & \multirow{2}{*}{ Obs. } & \multicolumn{4}{|c|}{ Fitted (estimated) background } \\
\hline & & & Total & $t \bar{t}$ & $W+$ jets & Other \\
\hline 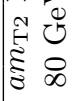 & $60<m_{\mathrm{T}}<90 \mathrm{GeV}+b$-veto & 1069 & $1067 \pm 33(1405)$ & $103 \pm 34$ & $890 \pm 50$ & $76 \pm 24$ \\
\hline \multirow{3}{*}{ 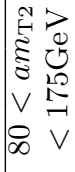 } & $60<m_{\mathrm{T}}<90 \mathrm{GeV}$ & 65 & $61 \pm 6 \quad(60)$ & $57 \pm 6 \quad(55)$ & $1.1 \pm 0.4$ & $3.1 \pm 1.1$ \\
\hline & $90<m_{\mathrm{T}}<120 \mathrm{GeV}$ & 17 & $16.8 \pm 2.1$ & $15.8 \pm 2.1(15)$ & $0.26 \pm 0.29$ & $0.76 \pm 0.30(0.9)$ \\
\hline & $m_{\mathrm{T}}>120 \mathrm{GeV}$ & 6 & $7.5 \pm 1.1$ & $6.6 \pm 1.2 \quad(7)$ & $0.11 \pm 0.11(0.2)$ & $0.71 \pm 0.27(0.8)$ \\
\hline \multirow{3}{*}{ 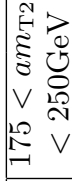 } & $60<m_{\mathrm{T}}<90 \mathrm{GeV}$ & 33 & $33 \pm 4 \quad(37)$ & $20.6 \pm 3.5(21)$ & $6.1 \pm 1.6$ & $5.9 \pm 2.0 \quad(7)$ \\
\hline & $90<m_{\mathrm{T}}<120 \mathrm{GeV}$ & 10 & $10.8 \pm 1.3$ & $7.7 \pm 1.2$ & $1.5 \pm 0.8$ & $1.6 \pm 0.6$ \\
\hline & $m_{\mathrm{T}}>120 \mathrm{GeV}$ & 10 & $7.0 \pm 1.0 \quad(7)$ & $4.6 \pm 0.9$ & $0.45 \pm 0.19$ & $1.9 \pm 0.6$ \\
\hline \multirow{3}{*}{ 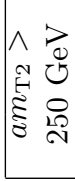 } & $60<m_{\mathrm{T}}<90 \mathrm{GeV}$ & 65 & $72 \pm 5 \quad(82)$ & $40 \pm 6 \quad(40)$ & $16 \pm 4$ & $15 \pm 5$ \\
\hline & $90<m_{\mathrm{T}}<120 \mathrm{GeV}$ & 16 & $18.2 \pm 2.3$ & $10.6 \pm 2.3$ & $3.5 \pm 1.4$ & $4.1 \pm 1.6$ \\
\hline & $m_{\mathrm{T}}>120 \mathrm{GeV}$ & 9 & $8.4 \pm 1.3 \quad(10)$ & $3.6 \pm 0.9$ & $2.2 \pm 0.8$ & $2.6 \pm 0.8$ \\
\hline
\end{tabular}

Table 18. Results of the all-bins background fit for the bCb_med2 analysis. The numbers in parenthesis are the pre-fit background estimates using the most accurate theoretical cross-sections available (cf. section 5).

\begin{tabular}{|c|c|c|c|c|c|c|}
\hline \multirow{2}{*}{\multicolumn{2}{|c|}{ bCd_bulk }} & \multirow{2}{*}{ Obs. } & \multicolumn{4}{|c|}{ Fitted (estimated) background } \\
\hline & & & Total & $t \bar{t}$ & $W+$ jets & Other \\
\hline \multirow{4}{*}{ 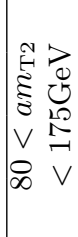 } & $60<m_{\mathrm{T}}<90 \mathrm{GeV}+b$-veto & 1345 & $1340 \pm 40(1702)$ & $270 \pm 60 \quad(260)$ & $990 \pm 80(1354)$ & $86 \pm 24 \quad(86)$ \\
\hline & $60<m_{\mathrm{T}}<90 \mathrm{GeV}$ & 2229 & $2220 \pm 90(2216)$ & $1920 \pm 90(1846)$ & $161 \pm 30$ & $150 \pm 40(150)$ \\
\hline & $90<m_{\mathrm{T}}<120 \mathrm{GeV}$ & 583 & $590 \pm 40 \quad(602)$ & $516 \pm 31$ & $30 \pm 6$ & $41 \pm 12$ \\
\hline & $m_{\mathrm{T}}>120 \mathrm{GeV}$ & 361 & $362 \pm 16 \quad(362)$ & $332 \pm 16$ & $8.8 \pm 2.4$ & $21 \pm 5$ \\
\hline \multirow{4}{*}{ 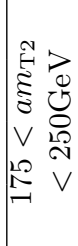 } & $60<m_{\mathrm{T}}<90 \mathrm{GeV}+b$-veto & 705 & $705 \pm 27 \quad(864)$ & $62 \pm 13 \quad(68)$ & $594 \pm 34 \quad(749)$ & $48 \pm 14$ \\
\hline & $60<m_{\mathrm{T}}<90 \mathrm{GeV}$ & 547 & $551 \pm 29 \quad(626)$ & $338 \pm 27 \quad(375)$ & $140 \pm 23$ & $74 \pm 19$ \\
\hline & $90<m_{\mathrm{T}}<120 \mathrm{GeV}$ & 144 & $141 \pm 13 \quad(152)$ & $93 \pm 10$ & $30 \pm 7 \quad(37)$ & $17 \pm 5$ \\
\hline & $m_{\mathrm{T}}>120 \mathrm{GeV}$ & 78 & $77 \pm 5 \quad(82)$ & $49 \pm 5$ & $12.4 \pm 3.5 \quad(15)$ & $15.3 \pm 3.0$ \\
\hline \multirow{4}{*}{ 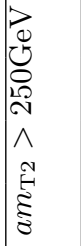 } & $60<m_{\mathrm{T}}<90 \mathrm{GeV}+b$-veto & 260 & $260 \pm 16$ & $17 \pm 5$ & $222 \pm 19$ & $21 \pm 7$ \\
\hline & $60<m_{\mathrm{T}}<90 \mathrm{GeV}$ & 241 & $239 \pm 13 \quad(285)$ & $117 \pm 19$ & $75 \pm 14 \quad(102)$ & $47 \pm 12$ \\
\hline & $90<m_{\mathrm{T}}<120 \mathrm{GeV}$ & 61 & $65 \pm 5 \quad(78)$ & $32 \pm 6$ & $20 \pm 4$ & $13 \pm 4$ \\
\hline & $m_{\mathrm{T}}>120 \mathrm{GeV}$ & 29 & $26.3 \pm 2.0$ & $10.5 \pm 2.2 \quad(12)$ & $7.0 \pm 1.5$ & $8.8 \pm 1.9$ \\
\hline
\end{tabular}

Table 19. Results of the all-bins background fit for the bCd_bulk analysis. The numbers in parenthesis are the pre-fit background estimates using the most accurate theoretical cross-sections available (cf. section 5). 


\begin{tabular}{|c|c|c|c|c|c|c|}
\hline \multirow{2}{*}{\multicolumn{2}{|c|}{ 3body }} & \multirow{2}{*}{ Obs. } & \multicolumn{4}{|c|}{ Fitted (estimated) background } \\
\hline & & & Total & $t \bar{t}$ & $W+$ jets & Other \\
\hline \multirow{4}{*}{ 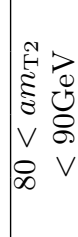 } & $60<m_{\mathrm{T}}<90 \mathrm{GeV}+b$-veto & 8 & $9.8 \pm 2.0 \quad(13)$ & $2.6 \pm 0.8$ & $6.3 \pm 2.0$ & $0.8 \pm 0.5 \quad(0.7)$ \\
\hline & $60<m_{\mathrm{T}}<90 \mathrm{GeV}$ & 3 & $5.9 \pm 0.8 \quad(6)$ & $5.1 \pm 0.8$ & $0.52 \pm 0.31(0.2)$ & $0.26 \pm 0.22(0.4)$ \\
\hline & $90<m_{\mathrm{T}}<120 \mathrm{GeV}$ & 12 & $14.6 \pm 1.7(14)$ & $12.0 \pm 1.7(11)$ & $1.6 \pm 0.7 \quad(2)$ & $1.0 \pm 0.6$ \\
\hline & $m_{\mathrm{T}}>120 \mathrm{GeV}$ & 8 & $7.3 \pm 1.3 \quad(7)$ & $6.5 \pm 1.3 \quad(7)$ & $0.13 \pm 0.20(0.1)$ & $0.69 \pm 0.18(0.5)$ \\
\hline \multirow{4}{*}{ 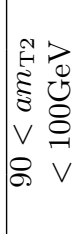 } & $60<m_{\mathrm{T}}<90 \mathrm{GeV}+b$-veto & 12 & $16.8 \pm 2.9$ & $4.1 \pm 1.1$ & $11.8 \pm 2.8$ & $1.0 \pm 0.6 \quad(0.8)$ \\
\hline & $60<m_{\mathrm{T}}<90 \mathrm{GeV}$ & 14 & $16.1 \pm 1.7$ & $13.1 \pm 1.6$ & $1.1 \pm 0.5 \quad(2)$ & $1.9 \pm 0.6$ \\
\hline & $90<m_{\mathrm{T}}<120 \mathrm{GeV}$ & 29 & $30.2 \pm 2.3(32)$ & $24.7 \pm 2.2(26)$ & $3.9 \pm 1.3$ & $1.6 \pm 1.2$ \\
\hline & $m_{\mathrm{T}}>120 \mathrm{GeV}$ & 22 & $26.0 \pm 2.1(26)$ & $22.9 \pm 2.3(23)$ & $1.1 \pm 0.6$ & $2.0 \pm 0.7$ \\
\hline \multirow{4}{*}{ 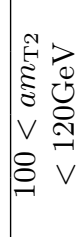 } & $60<m_{\mathrm{T}}<90 \mathrm{GeV}+b$-veto & 36 & $35 \pm 4 \quad(34)$ & $7.0 \pm 1.9$ & $26 \pm 4$ & $2.1 \pm 0.7$ \\
\hline & $60<m_{\mathrm{T}}<90 \mathrm{GeV}$ & 29 & $32.4 \pm 2.3(32)$ & $27.9 \pm 2.2(28)$ & $3.0 \pm 1.2$ & $1.5 \pm 0.9$ \\
\hline & $90<m_{\mathrm{T}}<120 \mathrm{GeV}$ & 57 & $59 \pm 4 \quad(61)$ & $51 \pm 4$ & $3.7 \pm 2.0$ & $3.9 \pm 2.1$ \\
\hline & $m_{\mathrm{T}}>120 \mathrm{GeV}$ & 74 & $70 \pm 5 \quad(66)$ & $62 \pm 6 \quad(58)$ & $4.4 \pm 1.4$ & $3.9 \pm 1.2$ \\
\hline \multirow{4}{*}{$\begin{array}{l}\hat{\text { N}} \\
\hat{\tilde{\xi}} \\
\hat{\Xi}\end{array}$} & $60<m_{\mathrm{T}}<90 \mathrm{GeV}+b$-veto & 114 & $112 \pm 10(115)$ & $14.4 \pm 3.3$ & $90 \pm 11$ & $8.0 \pm 1.9$ \\
\hline & $60<m_{\mathrm{T}}<90 \mathrm{GeV}$ & 108 & $88 \pm 5 \quad(82)$ & $66 \pm 5 \quad(62)$ & $16 \pm 4$ & $6.2 \pm 1.7$ \\
\hline & $90<m_{\mathrm{T}}<120 \mathrm{GeV}$ & 160 & $162 \pm 10(167)$ & $131 \pm 10$ & $20 \pm 5$ & $11 \pm 4$ \\
\hline & $m_{\mathrm{T}}>120 \mathrm{GeV}$ & 281 & $281 \pm 12(277)$ & $216 \pm 15(211)$ & $33 \pm 8$ & $32 \pm 7$ \\
\hline
\end{tabular}

Table 20. Results of the all-bins background fit for the 3body analysis. The numbers in parenthesis are the pre-fit background estimates using the most accurate theoretical cross-sections available (cf. section 5).

Open Access. This article is distributed under the terms of the Creative Commons Attribution License (CC-BY 4.0), which permits any use, distribution and reproduction in any medium, provided the original author(s) and source are credited.

\section{References}

[1] S. Weinberg, Implications of dynamical symmetry breaking, Phys. Rev. D 13 (1976) 974 [INSPIRE].

[2] E. Gildener, Gauge symmetry hierarchies, Phys. Rev. D 14 (1976) 1667 [InSPIRE].

[3] S. Weinberg, Implications of dynamical symmetry breaking: an addendum, Phys. Rev. D 19 (1979) 1277 [INSPIRE].

[4] L. Susskind, Dynamics of spontaneous symmetry breaking in the Weinberg-Salam theory, Phys. Rev. D 20 (1979) 2619 [InSPIRE].

[5] ATLAS collaboration, Observation of a new particle in the search for the standard model Higgs boson with the ATLAS detector at the LHC, Phys. Lett. B 716 (2012) 1 [arXiv:1207.7214] [INSPIRE]. 
[6] CMS collaboration, Observation of a new boson at a mass of $125 \mathrm{GeV}$ with the CMS experiment at the LHC, Phys. Lett. B 716 (2012) 30 [arXiv:1207.7235] [INSPIRE].

[7] L. Evans and P. Bryant, LHC machine, 2008 JINST 3 S08001 [InSPIRE].

[8] H. Miyazawa, Baryon number changing currents, Prog. Theor. Phys. 36 (1966) 1266 [INSPIRE].

[9] P. Ramond, Dual theory for free fermions, Phys. Rev. D 3 (1971) 2415 [inSPIRE].

[10] Y. Golfand and E.P. Likhtman, Extension of the algebra of Poincaré group generators and violation of p invariance, JETP Lett. 13 (1971) 323 [Pisma Zh. Eksp. Teor. Fiz. 13 (1971) 452] [INSPIRE].

[11] A. Neveu and J.H. Schwarz, Factorizable dual model of pions, Nucl. Phys. B 31 (1971) 86 [INSPIRE].

[12] A. Neveu and J.H. Schwarz, Quark model of dual pions, Phys. Rev. D 4 (1971) 1109 [INSPIRE].

[13] J.-L. Gervais and B. Sakita, Field theory interpretation of supergauges in dual models, Nucl. Phys. B 34 (1971) 632 [INSPIRE].

[14] D.V. Volkov and V.P. Akulov, Is the neutrino a Goldstone particle?, Phys. Lett. B 46 (1973) 109 [INSPIRE].

[15] J. Wess and B. Zumino, A Lagrangian model invariant under supergauge transformations, Phys. Lett. B 49 (1974) 52 [INSPIRE].

[16] J. Wess and B. Zumino, Supergauge transformations in four-dimensions, Nucl. Phys. B 70 (1974) 39 [INSPIRE].

[17] S. Dimopoulos and H. Georgi, Softly broken supersymmetry and SU(5), Nucl. Phys. B 193 (1981) 150 [INSPIRE].

[18] E. Witten, Dynamical breaking of supersymmetry, Nucl. Phys. B 188 (1981) 513 [inSPIRE].

[19] M. Dine, W. Fischler and M. Srednicki, Supersymmetric technicolor, Nucl. Phys. B 189 (1981) 575 [INSPIRE].

[20] S. Dimopoulos and S. Raby, Supercolor, Nucl. Phys. B 192 (1981) 353 [inSPIRE].

[21] N. Sakai, Naturalness in supersymmetric GUTS, Z. Phys. C 11 (1981) 153 [INSPIRE].

[22] R.K. Kaul and P. Majumdar, Cancellation of quadratically divergent mass corrections in globally supersymmetric spontaneously broken gauge theories, Nucl. Phys. B 199 (1982) 36 [INSPIRE].

[23] R. Barbieri and G.F. Giudice, Upper bounds on supersymmetric particle masses, Nucl. Phys. B 306 (1988) 63 [INSPIRE].

[24] B. de Carlos and J.A. Casas, One loop analysis of the electroweak breaking in supersymmetric models and the fine tuning problem, Phys. Lett. B 309 (1993) 320 [hep-ph/9303291] [INSPIRE].

[25] Super-Kamiokande collaboration, C. Regis et al., Search for proton decay via $p \rightarrow \mu^{+} K^{0}$ in Super-Kamiokande I, II and III, Phys. Rev. D 86 (2012) 012006 [arXiv:1205.6538] [INSPIRE].

[26] P. Fayet, Supersymmetry and weak, electromagnetic and strong interactions, Phys. Lett. B 64 (1976) 159 [INSPIRE]. 
[27] P. Fayet, Spontaneously broken supersymmetric theories of weak, electromagnetic and strong interactions, Phys. Lett. B 69 (1977) 489 [INSPIRE].

[28] G.R. Farrar and P. Fayet, Phenomenology of the production, decay and detection of new hadronic states associated with supersymmetry, Phys. Lett. B 76 (1978) 575 [INSPIRE].

[29] P. Fayet, Relations between the masses of the superpartners of leptons and quarks, the Goldstino couplings and the neutral currents, Phys. Lett. B 84 (1979) 416 [INSPIRE].

[30] LEP SUSY Working Group, ALEPH, DELPHI, L3 and OPAL collaborations, Combined LEP chargino results, up to $208 \mathrm{GeV}$ for large m0, LEPSUSYWG/01-03.1, (2001).

[31] MSSM Working Group collaboration, A. Djouadi et al., The minimal supersymmetric standard model: group summary report, hep-ph/9901246 [INSPIRE].

[32] ATLAS collaboration, Search for a supersymmetric partner to the top quark in final states with jets and missing transverse momentum at $\sqrt{s}=7 \mathrm{TeV}$ with the ATLAS detector, Phys. Rev. Lett. 109 (2012) 211802 [arXiv:1208.1447] [INSPIRE].

[33] ATLAS collaboration, Search for direct top squark pair production in final states with one isolated lepton, jets and missing transverse momentum in $\sqrt{s}=7 \mathrm{TeV}$ pp collisions using $4.7 \mathrm{fb}^{-1}$ of ATLAS data, Phys. Rev. Lett. 109 (2012) 211803 [arXiv:1208.2590] [INSPIRE].

[34] ATLAS collaboration, Search for light scalar top quark pair production in final states with two leptons with the ATLAS detector in $\sqrt{s}=7 \mathrm{TeV}$ proton-proton collisions, Eur. Phys. J. C 72 (2012) 2237 [arXiv: 1208.4305] [INSPIRE].

[35] ATLAS collaboration, Search for light top squark pair production in final states with leptons and b-jets with the ATLAS detector in $\sqrt{s}=7 \mathrm{TeV}$ proton-proton collisions, Phys. Lett. B 720 (2013) 13 [arXiv:1209.2102] [INSPIRE].

[36] ATLAS collaboration, Search for a heavy top-quark partner in final states with two leptons with the ATLAS detector at the LHC, JHEP 11 (2012) 094 [arXiv:1209.4186] [INSPIRE].

[37] ATLAS collaboration, Search for direct third-generation squark pair production in final states with missing transverse momentum and two b-jets in $\sqrt{s}=8 \mathrm{TeV}$ pp collisions with the ATLAS detector, JHEP 10 (2013) 189 [arXiv:1308.2631] [INSPIRE].

[38] ATLAS collaboration, Search for direct top-squark pair production in final states with two leptons in pp collisions at $\sqrt{s}=8 \mathrm{TeV}$ with the ATLAS detector, JHEP 06 (2014) 124 [arXiv: 1403.4853] [INSPIRE].

[39] CMS collaboration, Inclusive search for supersymmetry using the razor variables in pp collisions at $\sqrt{s}=7 \mathrm{TeV}$, Phys. Rev. Lett. 111 (2013) 081802 [arXiv:1212.6961] [INSPIRE].

[40] CMS collaboration, Search for supersymmetry in hadronic final states with missing transverse energy using the variables $\alpha_{T}$ and b-quark multiplicity in pp collisions at $\sqrt{s}=8 \mathrm{TeV}$, Eur. Phys. J. C 73 (2013) 2568 [arXiv:1303.2985] [InSPIRE].

[41] CMS collaboration, Search for top-squark pair production in the single-lepton final state in pp collisions at $\sqrt{s}=8 \mathrm{TeV}$, Eur. Phys. J. C 73 (2013) 2677 [arXiv:1308.1586] [inSPIRE].

[42] CMS collaboration, Search for top squark and higgsino production using diphoton Higgs boson decays, Phys. Rev. Lett. 112 (2014) 161802 [arXiv:1312.3310] [INSPIRE].

[43] CMS collaboration, Search for top-squark pairs decaying into Higgs or $Z$ bosons in pp collisions at $\sqrt{s}=8 \mathrm{TeV}$, Phys. Lett. B 736 (2014) 371 [arXiv:1405.3886] [INSPIRE]. 
[44] CDF collaboration, T. Aaltonen et al., Search for pair production of supersymmetric top quarks in dilepton events from $p \bar{p}$ collisions at $\sqrt{s}=1.96$ TeV, Phys. Rev. Lett. 104 (2010) 251801 [arXiv:0912.1308] [INSPIRE].

[45] D0 collaboration, V.M. Abazov et al., Search for the lightest scalar top quark in events with two leptons in $p \bar{p}$ collisions at $\sqrt{s}=1.96$ TeV, Phys. Lett. B 675 (2009) 289 [arXiv:0811.0459] [INSPIRE].

[46] LEP SUSY Working Group, ALEPH, DELPHI, L3 and OPAL collaborations, Combined LEP stop and sbottom results 183-208 GeV, LEPSUSYWG/04-02.1, (2001).

[47] ATLAS collaboration, Search for supersymmetry in pp collisions at $\sqrt{s}=7$ TeV in final states with missing transverse momentum and $b^{-}$jets with the ATLAS detector, Phys. Rev. D 85 (2012) 112006 [arXiv:1203.6193] [INSPIRE].

[48] ATLAS collaboration, Search for top and bottom squarks from gluino pair production in final states with missing transverse energy and at least three b-jets with the ATLAS detector, Eur. Phys. J. C 72 (2012) 2174 [arXiv:1207.4686] [InSPIRE].

[49] ATLAS collaboration, Search for new phenomena in final states with large jet multiplicities and missing transverse momentum at $\sqrt{s}=8$ TeV proton-proton collisions using the ATLAS experiment, JHEP 10 (2013) 130 [Erratum ibid. 01 (2014) 109] [arXiv:1308.1841] [INSPIRE].

[50] ATLAS collaboration, Search for supersymmetry at $\sqrt{s}=8 \mathrm{Te} V$ in final states with jets and two same-sign leptons or three leptons with the ATLAS detector, JHEP 06 (2014) 035 [arXiv:1404.2500] [INSPIRE].

[51] CMS collaboration, Search for supersymmetry in hadronic final states using $M_{\mathrm{T} 2}$ in $p p$ collisions at $\sqrt{s}=7$ TeV, JHEP 10 (2012) 018 [arXiv:1207.1798] [INSPIRE].

[52] CMS collaboration, Search for gluino mediated bottom- and top-squark production in multijet final states in pp collisions at 8 TeV, Phys. Lett. B 725 (2013) 243 [arXiv: 1305.2390] [INSPIRE].

[53] CMS collaboration, Search for supersymmetry in pp collisions at $\sqrt{s}=8$ TeV in events with a single lepton, large jet multiplicity and multiple b jets, Phys. Lett. B 733 (2014) 328 [arXiv:1311.4937] [INSPIRE].

[54] CMS collaboration, Search for new physics in events with same-sign dileptons and jets in pp collisions at $\sqrt{s}=8 \mathrm{TeV}$, JHEP 01 (2014) 163 [arXiv:1311.6736] [INSPIRE].

[55] CMS collaboration, Search for new physics in the multijet and missing transverse momentum final state in proton-proton collisions at $\sqrt{s}=8 \mathrm{TeV}$, JHEP 06 (2014) 055 [arXiv: 1402 . 4770] [INSPIRE].

[56] ATLAS collaboration, The ATLAS experiment at the CERN Large Hadron Collider, 2008 JINST 3 S08003 [INSPIRE].

[57] ATLAS collaboration, Performance of the ATLAS trigger system in 2010, Eur. Phys. J. C 72 (2012) 1849 [arXiv:1110.1530] [INSPIRE].

[58] ATLAS collaboration, Improved luminosity determination in pp collisions at $\sqrt{s}=7 \mathrm{TeV}$ using the ATLAS detector at the LHC, Eur. Phys. J. C 73 (2013) 2518 [arXiv:1302.4393] [INSPIRE].

[59] S. Frixione, P. Nason and C. Oleari, Matching NLO QCD computations with parton shower simulations: the POWHEG method, JHEP 11 (2007) 070 [arXiv:0709.2092] [INSPIRE]. 
[60] B.P. Kersevan and E. Richter-Was, The Monte Carlo event generator AcerMC versions 2.0 to 3.8 with interfaces to PYTHIA 6.4, HERWIG 6.5 and ARIADNE 4.1, Comput. Phys. Commun. 184 (2013) 919 [hep-ph/0405247] [INSPIRE].

[61] J. Alwall, M. Herquet, F. Maltoni, O. Mattelaer and T. Stelzer, MadGraph 5: going beyond, JHEP 06 (2011) 128 [arXiv:1106.0522] [INSPIRE].

[62] T. Gleisberg et al., Event generation with SHERPA 1.1, JHEP 02 (2009) 007 [arXiv:0811.4622] [INSPIRE].

[63] M. Bähr et al., HERWIG++ physics and manual, Eur. Phys. J. C 58 (2008) 639 [arXiv: 0803.0883] [INSPIRE].

[64] H.-L. Lai et al., New parton distributions for collider physics, Phys. Rev. D 82 (2010) 074024 [arXiv: 1007.2241] [INSPIRE].

[65] J. Pumplin et al., New generation of parton distributions with uncertainties from global QCD analysis, JHEP 07 (2002) 012 [hep-ph/0201195] [INSPIRE].

[66] ATLAS collaboration, New ATLAS event generator tunes to 2010 data, ATL-PHYS-PUB-2011-008, CERN, Geneva Switzerland (2011).

[67] P.Z. Skands, Tuning Monte Carlo generators: the Perugia tunes, Phys. Rev. D 82 (2010) 074018 [arXiv: 1005.3457] [INSPIRE].

[68] S. Gieseke, C. Rohr and A. Siodmok, Colour reconnections in Herwigt+, Eur. Phys. J. C 72 (2012) 2225 [arXiv: 1206.0041] [InSPIRE].

[69] T. Sjöstrand, S. Mrenna and P.Z. Skands, PYTHIA 6.4 physics and manual, JHEP 05 (2006) 026 [hep-ph/0603175] [inSPIRE].

[70] ATLAS collaboration, The ATLAS simulation infrastructure, Eur. Phys. J. C 70 (2010) 823 [arXiv: 1005.4568] [INSPIRE].

[71] GEANT4 collaboration, S. Agostinelli et al., GEANT4: a simulation toolkit, Nucl. Instrum. Meth. A 506 (2003) 250 [INSPIRE].

[72] ATLAS collaboration, The simulation principle and performance of the ATLAS fast calorimeter simulation FastCaloSim, ATL-PHYS-PUB-2010-013, CERN, Geneva Switzerland (2010).

[73] T. Sjöstrand, S. Mrenna and P.Z. Skands, A brief introduction to PYTHIA 8.1, Comput. Phys. Commun. 178 (2008) 852 [arXiv:0710.3820] [INSPIRE].

[74] ATLAS collaboration, Measurement of top-quark pair differential cross-sections in the $\ell+j e t s$ channel in pp collisions at $\sqrt{s}=7 \mathrm{TeV}$ using the ATLAS detector, ATLAS-CONF-2013-099, CERN, Geneva Switzerland (2013).

[75] S. Frixione, E. Laenen, P. Motylinski, B.R. Webber and C.D. White, Single-top hadroproduction in association with a W boson, JHEP 07 (2008) 029 [arXiv:0805.3067] [INSPIRE].

[76] M. Czakon, P. Fiedler and A. Mitov, Total top-quark pair-production cross section at hadron colliders through $O\left(\alpha_{S}^{4}\right)$, Phys. Rev. Lett. 110 (2013) 252004 [arXiv:1303.6254] [INSPIRE].

[77] M. Czakon and A. Mitov, NNLO corrections to top pair production at hadron colliders: the quark-gluon reaction, JHEP 01 (2013) 080 [arXiv: 1210.6832] [INSPIRE].

[78] M. Czakon and A. Mitov, NNLO corrections to top-pair production at hadron colliders: the all-fermionic scattering channels, JHEP 12 (2012) 054 [arXiv:1207.0236] [INSPIRE]. 
[79] P. Bärnreuther, M. Czakon and A. Mitov, Percent level precision physics at the Tevatron: first genuine NNLO QCD corrections to $q \bar{q} \rightarrow t \bar{t}+X$, Phys. Rev. Lett. 109 (2012) 132001 [arXiv:1204.5201] [INSPIRE].

[80] M. Cacciari, M. Czakon, M. Mangano, A. Mitov and P. Nason, Top-pair production at hadron colliders with next-to-next-to-leading logarithmic soft-gluon resummation, Phys. Lett. B $\mathbf{7 1 0 ~ ( 2 0 1 2 )} 612$ [arXiv:1111.5869] [INSPIRE].

[81] M. Czakon and A. Mitov, Top++: a program for the calculation of the top-pair cross-section at hadron colliders, Comput. Phys. Commun. 185 (2014) 2930 [arXiv:1112.5675] [INSPIRE].

[82] N. Kidonakis, Next-to-next-to-leading-order collinear and soft gluon corrections for t-channel single top quark production, Phys. Rev. D 83 (2011) 091503 [arXiv:1103.2792] [INSPIRE].

[83] N. Kidonakis, Two-loop soft anomalous dimensions for single top quark associated production with a $W^{-}$or $H^{-}$, Phys. Rev. D 82 (2010) 054018 [arXiv:1005.4451] [INSPIRE].

[84] N. Kidonakis, NNLL resummation for s-channel single top quark production, Phys. Rev. D 81 (2010) 054028 [arXiv:1001.5034] [INSPIRE].

[85] J.M. Campbell and R.K. Ellis, $t \bar{t} W^{ \pm}$production and decay at NLO, JHEP 07 (2012) 052 [arXiv: 1204.5678] [INSPIRE].

[86] M.V. Garzelli, A. Kardos, C.G. Papadopoulos and Z. Trócsányi, $t \bar{t} W^{ \pm}$and $t \bar{t} Z$ hadroproduction at NLO accuracy in QCD with parton shower and hadronization effects, JHEP 11 (2012) 056 [arXiv:1208.2665] [INSPIRE].

[87] J. Campbell, R.K. Ellis and R. Röntsch, Single top production in association with a $Z$ boson at the LHC, Phys. Rev. D 87 (2013) 114006 [arXiv:1302.3856] [InSPIRE].

[88] S. Catani, L. Cieri, G. Ferrera, D. de Florian and M. Grazzini, Vector boson production at hadron colliders: a fully exclusive QCD calculation at NNLO, Phys. Rev. Lett. 103 (2009) 082001 [arXiv: 0903.2120] [INSPIRE].

[89] J.M. Campbell and R.K. Ellis, An update on vector boson pair production at hadron colliders, Phys. Rev. D 60 (1999) 113006 [hep-ph/9905386] [INSPIRE].

[90] J.M. Campbell, R.K. Ellis and C. Williams, Vector boson pair production at the LHC, JHEP 07 (2011) 018 [arXiv:1105.0020] [InSPIRE].

[91] A.D. Martin, W.J. Stirling, R.S. Thorne and G. Watt, Parton distributions for the LHC, Eur. Phys. J. C 63 (2009) 189 [arXiv:0901.0002] [INSPIRE].

[92] P.M. Nadolsky et al., Implications of CTEQ global analysis for collider observables, Phys. Rev. D 78 (2008) 013004 [arXiv:0802.0007] [INSPIRE].

[93] W. Beenakker, M. Krämer, T. Plehn, M. Spira and P.M. Zerwas, Stop production at hadron colliders, Nucl. Phys. B 515 (1998) 3 [hep-ph/9710451] [InSPIRE].

[94] W. Beenakker et al., Supersymmetric top and bottom squark production at hadron colliders, JHEP 08 (2010) 098 [arXiv: 1006.4771] [InSPIRE].

[95] W. Beenakker et al., Squark and gluino hadroproduction, Int. J. Mod. Phys. A 26 (2011) 2637 [arXiv: 1105.1110] [INSPIRE].

[96] M. Krämer et al., Supersymmetry production cross sections in pp collisions at $\sqrt{s}=7 \mathrm{TeV}$, arXiv: 1206.2892 [INSPIRE]. 
[97] M. Cahill-Rowley, J.L. Hewett, A. Ismail and T.G. Rizzo, pMSSM studies at the 7, 8 and $14 \mathrm{TeV} L H C$, arXiv: 1307.8444 [INSPIRE].

[98] WMAP collaboration, G. Hinshaw et al., Nine-year Wilkinson Microwave Anisotropy Probe (WMAP) observations: cosmological parameter results, Astrophys. J. Suppl. 208 (2013) 19 [arXiv:1212.5226] [INSPIRE].

[99] J.R. Ellis, K. Enqvist, D.V. Nanopoulos and F. Zwirner, Observables in low-energy superstring models, Mod. Phys. Lett. A 1 (1986) 57 [inSPIRE].

[100] ATLAS collaboration, Performance of primary vertex reconstruction in proton-proton collisions at $\sqrt{s}=7 \mathrm{TeV}$ in the ATLAS experiment, ATLAS-CONF-2010-069, CERN, Geneva Switzerland (2010).

[101] W. Lampl et al., Calorimeter clustering algorithms : description and performance, ATL-LARG-PUB-2008-002, CERN, Geneva Switzerland (2008).

[102] M. Cacciari, G.P. Salam and G. Soyez, The anti-kt jet clustering algorithm, JHEP 04 (2008) 063 [arXiv: 0802.1189] [InSPIRE].

[103] M. Cacciari and G.P. Salam, Dispelling the $N^{3}$ myth for the $k_{t}$ jet-finder, Phys. Lett. B 641 (2006) 57 [hep-ph/0512210] [INSPIRE].

[104] M. Cacciari, G.P. Salam and G. Soyez, The catchment area of jets, JHEP 04 (2008) 005 [arXiv:0802.1188] [INSPIRE].

[105] ATLAS collaboration, Jet energy measurement with the ATLAS detector in proton-proton collisions at $\sqrt{s}=7 \mathrm{TeV}$, Eur. Phys. J. C 73 (2013) 2304 [arXiv:1112.6426] [InSPIRE].

[106] ATLAS collaboration, Jet energy measurement and its systematic uncertainty in proton-proton collisions at $\sqrt{s}=7 \mathrm{TeV}$ with the ATLAS detector, arXiv:1406.0076 [INSPIRE].

[107] ATLAS collaboration, Performance of jet substructure techniques for large- $R$ jets in proton-proton collisions at $\sqrt{s}=7 \mathrm{TeV}$ using the ATLAS detector, JHEP 09 (2013) 076 [arXiv: 1306.4945] [INSPIRE].

[108] D. Krohn, J. Thaler and L.-T. Wang, Jet trimming, JHEP 02 (2010) 084 [arXiv:0912.1342] [INSPIRE].

[109] ATLAS collaboration, Commissioning of the ATLAS high-performance b-tagging algorithms in the $7 \mathrm{TeV}$ collision data, ATLAS-CONF-2011-102, CERN, Geneva Switzerland (2011).

[110] ATLAS collaboration, Calibrating the b-tag efficiency and mistag rate in $35 \mathrm{pb}^{-1}$ of data with the ATLAS detector, ATLAS-CONF-2011-089, CERN, Geneva Switzerland (2011).

[111] ATLAS collaboration, Measurement of the mistag rate with $5 \mathrm{fb}^{-1}$ of data collected by the ATLAS detector, ATLAS-CONF-2012-040, CERN, Geneva Switzerland (2012).

[112] ATLAS collaboration, Electron performance measurements with the ATLAS detector using the 2010 LHC proton-proton collision data, Eur. Phys. J. C 72 (2012) 1909 [arXiv:1110.3174] [INSPIRE].

[113] ATLAS collaboration, Electron reconstruction and identification efficiency measurements with the ATLAS detector using the 2011 LHC proton-proton collision data, Eur. Phys. J. C 74 (2014) 2941 [arXiv:1404.2240] [INSPIRE]. 
[114] ATLAS collaboration, Electron efficiency measurements with the ATLAS detector using the 2012 LHC proton-proton collision data, ATLAS-CONF-2014-032, CERN, Geneva Switzerland (2014).

[115] ATLAS collaboration, Muon reconstruction efficiency in reprocessed 2010 LHC proton-proton collision data recorded with the ATLAS detector, ATLAS-CONF-2011-063, CERN, Geneva Switzerland (2011).

[116] ATLAS collaboration, Preliminary results on the muon reconstruction efficiency, momentum resolution and momentum scale in ATLAS 2012 pp collision data, ATLAS-CONF-2013-088, CERN, Geneva Switzerland (2013).

[117] ATLAS collaboration, Muon reconstruction efficiency and momentum resolution of the ATLAS experiment in proton-proton collisions at $\sqrt{s}=7 \mathrm{TeV}$ in 2010, Eur. Phys. J. C 74 (2014) 3034 [arXiv: 1404.4562] [INSPIRE].

[118] ATLAS collaboration, Measurements of the photon identification efficiency with the ATLAS detector using $4.9 \mathrm{fb}^{-1}$ of pp collision data collected in 2011, ATLAS-CONF-2012-123, CERN, Geneva Switzerland (2012).

[119] ATLAS collaboration, Measurement of the inclusive isolated prompt photon cross section in pp collisions at $\sqrt{s}=7 \mathrm{TeV}$ with the ATLAS detector, Phys. Rev. D 83 (2011) 052005 [arXiv: 1012.4389] [INSPIRE].

[120] ATLAS collaboration, Performance of the reconstruction and identification of hadronic $\tau$ decays with ATLAS, ATLAS-CONF-2011-152, CERN, Geneva Switzerland (2011).

[121] ATLAS collaboration, Performance of the reconstruction and identification of hadronic $\tau$ decays in ATLAS with 2011 data, ATLAS-CONF-2012-142, CERN, Geneva Switzerland (2012).

[122] ATLAS collaboration, Performance of missing transverse momentum reconstruction in proton-proton collisions at $7 \mathrm{TeV}$ with ATLAS, Eur. Phys. J. C 72 (2012) 1844 [arXiv:1108.5602] [INSPIRE].

[123] ATLAS collaboration, Performance of missing transverse momentum reconstruction in ATLAS studied in proton-proton collisions recorded in 2012 at $8 \mathrm{TeV}$, ATLAS-CONF-2013-082, CERN, Geneva Switzerland (2013).

[124] C.G. Lester and D.J. Summers, Measuring masses of semi-invisibly decaying particles pair produced at hadron colliders, Phys. Lett. B 463 (1999) 99 [hep-ph/9906349] [INSPIRE].

[125] Y. Bai, H.-C. Cheng, J. Gallicchio and J. Gu, Stop the top background of the stop search, JHEP 07 (2012) 110 [arXiv:1203.4813] [INSPIRE].

[126] A.J. Barr, B. Gripaios and C.G. Lester, Transverse masses and kinematic constraints: from the boundary to the crease, JHEP 11 (2009) 096 [arXiv:0908.3779] [INSPIRE].

[127] P. Konar, K. Kong, K.T. Matchev and M. Park, Dark matter particle spectroscopy at the LHC: generalizing $M_{\mathrm{T} 2}$ to asymmetric event topologies, JHEP 04 (2010) 086 [arXiv:0911.4126] [INSPIRE].

[128] M.L. Graesser and J. Shelton, Hunting mixed top squark decays, Phys. Rev. Lett. 111 (2013) 121802 [arXiv:1212.4495] [INSPIRE].

[129] ATLAS collaboration, Jet energy resolution in proton-proton collisions at $\sqrt{s}=7 \mathrm{TeV}$ recorded in 2010 with the ATLAS detector, Eur. Phys. J. C 73 (2013) 2306 [arXiv: 1210.6210$]$ [INSPIRE]. 
[130] ATLAS collaboration, Further search for supersymmetry at $\sqrt{s}=7 \mathrm{TeV}$ in final states with jets, missing transverse momentum and isolated leptons with the ATLAS detector, Phys. Rev. D 86 (2012) 092002 [arXiv: 1208.4688] [INSPIRE].

[131] ATLAS collaboration, Search for supersymmetry in final states with jets, missing transverse momentum and one isolated lepton in $\sqrt{s}=7 \mathrm{TeV}$ pp collisions using $1 \mathrm{fb}^{-1}$ of ATLAS data, Phys. Rev. D 85 (2012) 012006 [arXiv:1109.6606] [InSPIRE].

[132] K. Melnikov, M. Schulze and A. Scharf, QCD corrections to top quark pair production in association with a photon at hadron colliders, Phys. Rev. D 83 (2011) 074013 [arXiv: 1102.1967] [INSPIRE].

[133] ATLAS collaboration, Jet mass and substructure of inclusive jets in $\sqrt{s}=7 \mathrm{TeV} p p$ collisions with the ATLAS experiment, JHEP 05 (2012) 128 [arXiv:1203.4606] [INSPIRE].

[134] ATLAS collaboration, Calibration of b-tagging using dileptonic top pair events in a combinatorial likelihood approach with the ATLAS experiment, ATLAS-CONF-2014-004, CERN, Geneva Switzerland (2014).

[135] ATLAS collaboration, Measurement of the b-tag efficiency in a sample of jets containing muons with $5 \mathrm{fb}^{-1}$ of data from the ATLAS detector, ATLAS-CONF-2012-043, CERN, Geneva Switzerland (2012).

[136] ATLAS collaboration, b-jet tagging calibration on $c$-jets containing $D^{*+}$ mesons, ATLAS-CONF-2012-039, CERN, Geneva Switzerland (2012).

[137] M. Botje et al., The PDF4LHC working group interim recommendations, arXiv:1101.0538 [INSPIRE].

[138] R.D. Ball et al., Parton distributions with LHC data, Nucl. Phys. B 867 (2013) 244 [arXiv:1207.1303] [INSPIRE].

[139] J.M. Butterworth, J.R. Forshaw and M.H. Seymour, Multiparton interactions in photoproduction at HERA, Z. Phys. C 72 (1996) 637 [hep-ph/9601371] [INSPIRE].

[140] ATLAS collaboration, Measurement of $t \bar{t}$ production with a veto on additional central jet activity in pp collisions at $\sqrt{s}=7 \mathrm{TeV}$ using the ATLAS detector, Eur. Phys. J. C 72 (2012) 2043 [arXiv: 1203.5015] [INSPIRE].

[141] ATLAS collaboration, Measurement of the cross-section for $W$ boson production in association with b-jets in pp collisions at $\sqrt{s}=7 \mathrm{TeV}$ with the ATLAS detector, JHEP 06 (2013) 084 [arXiv: 1302.2929] [INSPIRE].

[142] G. Cowan, K. Cranmer, E. Gross and O. Vitells, Asymptotic formulae for likelihood-based tests of new physics, Eur. Phys. J. C 71 (2011) 1554 [arXiv:1007.1727] [INSPIRE].

[143] A.L. Read, Presentation of search results: the CLs technique, J. Phys. G 28 (2002) 2693 [INSPIRE].

[144] ALEPH collaboration, A. Heister et al., Search for scalar quarks in $e^{+} e^{-}$collisions at $\sqrt{s}$ up to $209 \mathrm{GeV}$, Phys. Lett. B 537 (2002) 5 [hep-ex/0204036] [INSPIRE].

[145] B. Nachman and C.G. Lester, Significance variables, Phys. Rev. D 88 (2013) 075013 [arXiv: 1303.7009] [INSPIRE]. 


\section{The ATLAS collaboration}

G. $\operatorname{Aad}^{84}$, B. Abbott ${ }^{112}$, J. Abdallah ${ }^{152}$, S. Abdel Khalek ${ }^{116}$, O. Abdinov ${ }^{11}$, R. Aben ${ }^{106}$, B. Abi ${ }^{113}$, M. Abolins ${ }^{89}$, O.S. AbouZeid ${ }^{159}$, H. Abramowicz ${ }^{154}$, H. Abreu ${ }^{153}$, R. Abreu ${ }^{30}$, Y. Abulaiti ${ }^{147 a, 147 b}$, B.S. Acharya ${ }^{165 a, 165 b, a}$, L. Adamczyk ${ }^{38 a}$, D.L. Adams ${ }^{25}$, J. Adelman ${ }^{177}$, S. Adomeit ${ }^{99}$, T. Adye ${ }^{130}$, T. Agatonovic-Jovin ${ }^{13 a}$, J.A. Aguilar-Saavedra ${ }^{125 a, 125 f}$, M. Agustoni ${ }^{17}$, S.P. Ahlen ${ }^{22}$, F. Ahmadov ${ }^{64, b}$, G. Aielli ${ }^{134 a, 134 b}$, H. Akerstedt ${ }^{147 a, 147 b}$, T.P.A. Åkesson ${ }^{80}$, G. Akimoto ${ }^{156}$, A.V. Akimov ${ }^{95}$, G.L. Alberghi ${ }^{20 a, 20 b}$, J. Albert ${ }^{170}$, S. Albrand ${ }^{55}$, M.J. Alconada Verzini ${ }^{70}$, M. Aleksa ${ }^{30}$, I.N. Aleksandrov ${ }^{64}$, C. Alexa ${ }^{26 a}$, G. Alexander ${ }^{154}$, G. Alexandre ${ }^{49}$, T. Alexopoulos ${ }^{10}$, M. Alhroob ${ }^{165 a, 165 c}$, G. Alimonti ${ }^{90 a}$, L. Alio ${ }^{84}$, J. Alison ${ }^{31}$, B.M.M. Allbrooke ${ }^{18}$, L.J. Allison ${ }^{71}$, P.P. Allport ${ }^{73}$, J. Almond ${ }^{83}$, A. Aloisio 103a,103b , A. Alonso ${ }^{36}$, F. Alonso ${ }^{70}$, C. Alpigiani ${ }^{75}$, A. Altheimer ${ }^{35}$, B. Alvarez Gonzalez ${ }^{89}$, M.G. Alviggi ${ }^{103 a, 103 b}$,

K. Amako ${ }^{65}$, Y. Amaral Coutinho ${ }^{24 a}$, C. Amelung ${ }^{23}$, D. Amidei ${ }^{88}$, S.P. Amor Dos Santos ${ }^{125 a, 125 c}$, A. Amorim $125 \mathrm{a}, 125 \mathrm{~b}, \mathrm{~S}$. Amoroso ${ }^{48}$, N. Amram ${ }^{154}$, G. Amundsen ${ }^{23}$, C. Anastopoulos ${ }^{140}$, L.S. Ancu ${ }^{49}$, N. Andari ${ }^{30}$, T. Andeen ${ }^{35}$, C.F. Anders ${ }^{58 b}$, G. Anders ${ }^{30}$, K.J. Anderson ${ }^{31}$, A. Andreazza ${ }^{90 a, 90 b}$, V. Andrei ${ }^{58 a}$, X.S. Anduaga ${ }^{70}$, S. Angelidakis ${ }^{9}$, I. Angelozzi ${ }^{106}$, P. Anger ${ }^{44}$, A. Angerami ${ }^{35}$, F. Anghinolfi ${ }^{30}$, A.V. Anisenkov ${ }^{108}$, N. Anjos ${ }^{125 a}$, A. Annovi ${ }^{47}$, A. Antonaki ${ }^{9}$, M. Antonelli ${ }^{47}$, A. Antonov ${ }^{97}$, J. Antos ${ }^{145 b}$, F. Anulli ${ }^{133 a}$, M. Aoki ${ }^{65}$, L. Aperio Bella ${ }^{18}$, R. Apolle ${ }^{119, c}$, G. Arabidze ${ }^{89}$, I. Aracena ${ }^{144}$, Y. Arai ${ }^{65}$, J.P. Araque ${ }^{125 a}$, A.T.H. Arce ${ }^{45}$, J-F. Arguin ${ }^{94}$, S. Argyropoulos ${ }^{42}$, M. Arik ${ }^{19 a}$, A.J. Armbruster ${ }^{30}$, O. Arnaez ${ }^{30}$, V. Arnal ${ }^{81}$,

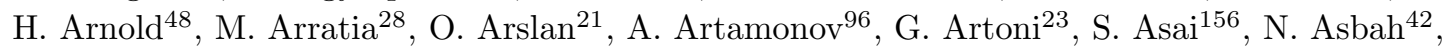
A. Ashkenazi ${ }^{154}$, B. Åsman 147a,147b, L. Asquith 6 , K. Assamagan 25 , R. Astalos ${ }^{145 a}$,

M. Atkinson ${ }^{166}$, N.B. Atlay ${ }^{142}$, B. Auerbach ${ }^{6}$, K. Augsten ${ }^{127}$, M. Aurousseau ${ }^{146 b}$, G. Avolio ${ }^{30}$, G. Azuelos ${ }^{94, d}$, Y. Azuma ${ }^{156}$, M.A. Baak ${ }^{30}$, A. Baas ${ }^{58 a}$, C. Bacci ${ }^{135 a, 135 b}$, H. Bachacou ${ }^{137}$, K. Bachas ${ }^{155}$, M. Backes ${ }^{30}$, M. Backhaus ${ }^{30}$, J. Backus Mayes ${ }^{144}$, E. Badescu ${ }^{26 a}$, P. Bagiacchi ${ }^{133 a, 133 b}$, P. Bagnaia ${ }^{133 a, 133 b}$, Y. Bai ${ }^{33 a}$, T. Bain ${ }^{35}$, J.T. Baines ${ }^{130}$, O.K. Baker ${ }^{177}$, P. Balek ${ }^{128}$, F. Balli ${ }^{137}$, E. Banas ${ }^{39}$, Sw. Banerjee ${ }^{174}$, A.A.E. Bannoura ${ }^{176}$, V. Bansal ${ }^{170}$, H.S. Bansil ${ }^{18}$, L. Barak ${ }^{173}$, S.P. Baranov ${ }^{95}$, E.L. Barberio ${ }^{87}$, D. Barberis ${ }^{50 a, 50 b}$, M. Barbero ${ }^{84}$, T. Barillari ${ }^{100}$, M. Barisonzi ${ }^{176}$, T. Barklow ${ }^{144}$, N. Barlow ${ }^{28}$, B.M. Barnett ${ }^{130}$, R.M. Barnett ${ }^{15}$, Z. Barnovska ${ }^{5}$, A. Baroncelli ${ }^{135 a}$, G. Barone ${ }^{49}$, A.J. Barr ${ }^{119}$, F. Barreiro ${ }^{81}$,

J. Barreiro Guimarães da Costa ${ }^{57}$, R. Bartoldus ${ }^{144}$, A.E. Barton ${ }^{71}$, P. Bartos ${ }^{145 a}$, V. Bartsch ${ }^{150}$, A. Bassalat ${ }^{116}$, A. Basye ${ }^{166}$, R.L. Bates ${ }^{53}$, J.R. Batley ${ }^{28}$, M. Battaglia ${ }^{138}$, M. Battistin ${ }^{30}$,

F. Bauer ${ }^{137}$, H.S. Bawa ${ }^{144, e}$, M.D. Beattie ${ }^{71}$, T. Beau ${ }^{79}$, P.H. Beauchemin ${ }^{162}$,

R. Beccherle ${ }^{123 a, 123 b}$, P. Bechtle ${ }^{21}$, H.P. Beck ${ }^{17}$, K. Becker ${ }^{176}$, S. Becker ${ }^{99}$, M. Beckingham ${ }^{171}$, C. Becot $^{116}$, A.J. Beddall ${ }^{19 c}$, A. Beddall ${ }^{19 c}$, S. Bedikian ${ }^{177}$, V.A. Bednyakov ${ }^{64}$, C.P. Bee ${ }^{149}$, L.J. Beemster ${ }^{106}$, T.A. Beermann ${ }^{176}$, M. Begel ${ }^{25}$, K. Behr ${ }^{119}$, C. Belanger-Champagne ${ }^{86}$, P.J. Bell ${ }^{49}$, W.H. Bell ${ }^{49}$, G. Bella ${ }^{154}$, L. Bellagamba ${ }^{20 a}$, A. Bellerive $^{29}$, M. Bellomo ${ }^{85}$,

K. Belotskiy ${ }^{97}$, O. Beltramello ${ }^{30}$, O. Benary ${ }^{154}$, D. Benchekroun ${ }^{136 a}$, K. Bendtz ${ }^{147 a, 147 b}$, N. Benekos ${ }^{166}$, Y. Benhammou ${ }^{154}$, E. Benhar Noccioli ${ }^{49}$, J.A. Benitez Garcia ${ }^{160 b}$, D.P. Benjamin ${ }^{45}$, J.R. Bensinger ${ }^{23}$, K. Benslama ${ }^{131}$, S. Bentvelsen ${ }^{106}$, D. Berge ${ }^{106}$, E. Bergeaas Kuutmann ${ }^{16}$, N. Berger ${ }^{5}$, F. Berghaus ${ }^{170}$, J. Beringer ${ }^{15}$, C. Bernard ${ }^{22}$, P. Bernat ${ }^{77}$, C. Bernius ${ }^{78}$, F.U. Bernlochner ${ }^{170}$, T. Berry ${ }^{76}$, P. Berta ${ }^{128}$, C. Bertella ${ }^{84}$, G. Bertoli ${ }^{147 a, 147 b}$, F. Bertolucci ${ }^{123 a, 123 b}$, C. Bertsche ${ }^{112}$, D. Bertsche ${ }^{112}$, M.I. Besana ${ }^{90 a}$, G.J. Besjes ${ }^{105}$, O. Bessidskaia ${ }^{147 a, 147 b}$, M. Bessner ${ }^{42}$, N. Besson ${ }^{137}$, C. Betancourt ${ }^{48}$, S. Bethke ${ }^{100}$, W. Bhimji ${ }^{46}$, R.M. Bianchi ${ }^{124}$, L. Bianchini ${ }^{23}$, M. Bianco ${ }^{30}$, O. Biebel ${ }^{99}$, S.P. Bieniek ${ }^{77}$, K. Bierwagen ${ }^{54}$, J. Biesiada ${ }^{15}$, M. Biglietti ${ }^{135 a}$, J. Bilbao De Mendizabal ${ }^{49}$, H. Bilokon ${ }^{47}$, M. Bindi ${ }^{54}$, S. Binet ${ }^{116}$, A. Bingul ${ }^{19 c}$, C. Bini ${ }^{133 a, 133 b}$, C.W. Black ${ }^{151}$, J.E. Black ${ }^{144}$, K.M. Black ${ }^{22}$, D. Blackburn ${ }^{139}$, R.E. Blair ${ }^{6}$, J.-B. Blanchard ${ }^{137}$, T. Blazek ${ }^{145 a}$, I. Bloch ${ }^{42}$, C. Blocker ${ }^{23}$, W. Blum ${ }^{82, *}$, 
U. Blumenschein ${ }^{54}$, G.J. Bobbink ${ }^{106}$, V.S. Bobrovnikov ${ }^{108}$, S.S. Bocchetta ${ }^{80}$, A. Bocci ${ }^{45}$, C. Bock $^{99}$, C.R. Boddy ${ }^{119}$, M. Boehler ${ }^{48}$, T.T. Boek ${ }^{176}$, J.A. Bogaerts ${ }^{30}$, A.G. Bogdanchikov ${ }^{108}$, A. Bogouch ${ }^{11 *}$, C. Bohm ${ }^{147 a}$, J. Bohm ${ }^{126}$, V. Boisvert ${ }^{76}$, T. Bold ${ }^{38 a}$, V. Boldea ${ }^{26 a}$, A.S. Boldyrev ${ }^{98}$, M. Bomben ${ }^{79}$, M. Bona ${ }^{75}$, M. Boonekamp $^{137}$, A. Borisov ${ }^{129}$, G. Borissov ${ }^{71}$, M. Borri ${ }^{83}$, S. Borroni ${ }^{42}$, J. Bortfeldt ${ }^{99}$, V. Bortolotto ${ }^{135 a, 135 b}$, K. Bos ${ }^{106}$, D. Boscherini ${ }^{20 a}$, M. Bosman ${ }^{12}$, H. Boterenbrood ${ }^{106}$, J. Boudreau ${ }^{124}$, J. Bouffard ${ }^{2}$, E.V. Bouhova-Thacker ${ }^{71}$, D. Boumediene ${ }^{34}$, C. Bourdarios ${ }^{116}$, N. Bousson ${ }^{113}$, S. Boutouil ${ }^{136 \mathrm{~d}}$, A. Boveia ${ }^{31}$, J. Boyd ${ }^{30}$, I.R. Boyko ${ }^{64}$, J. Bracinik ${ }^{18}$, A. Brandt ${ }^{8}$, G. Brandt ${ }^{15}$, O. Brandt ${ }^{58 a}$, U. Bratzler ${ }^{157}$, B. Brau ${ }^{85}$, J.E. Brau ${ }^{115}$, H.M. Braun ${ }^{176, *}$, S.F. Brazzale ${ }^{165 a, 165 c}$, B. Brelier ${ }^{159}$, K. Brendlinger ${ }^{121}$, A.J. Brennan ${ }^{87}$, R. Brenner ${ }^{167}$, S. Bressler ${ }^{173}$, K. Bristow ${ }^{146 c}$, T.M. Bristow ${ }^{46}$, D. Britton ${ }^{53}$, F.M. Brochu ${ }^{28}$, I. Brock ${ }^{21}$, R. Brock ${ }^{89}$, C. Bromberg ${ }^{89}$, J. Bronner ${ }^{100}$, G. Brooijmans ${ }^{35}$, T. Brooks ${ }^{76}$, W.K. Brooks ${ }^{32 b}$, J. Brosamer ${ }^{15}$, E. Brost ${ }^{115}$, J. Brown ${ }^{55}$,

P.A. Bruckman de Renstrom ${ }^{39}$, D. Bruncko ${ }^{145 b}$, R. Bruneliere ${ }^{48}$, S. Brunet $^{60}$, A. Bruni ${ }^{20 a}$, G. Bruni ${ }^{20 a}$, M. Bruschi ${ }^{20 a}$, L. Bryngemark ${ }^{80}$, T. Buanes ${ }^{14}$, Q. Buat ${ }^{143}$, F. Bucci $^{49}$, P. Buchholz ${ }^{142}$, R.M. Buckingham ${ }^{119}$, A.G. Buckley ${ }^{53}$, S.I. Buda ${ }^{26 a}$, I.A. Budagov ${ }^{64}$, F. Buehrer ${ }^{48}$, L. Bugge ${ }^{118}$, M.K. Bugge ${ }^{118}$, O. Bulekov ${ }^{97}$, A.C. Bundock ${ }^{73}$, H. Burckhart ${ }^{30}$, S. Burdin ${ }^{73}$, B. Burghgrave ${ }^{107}$, S. Burke ${ }^{130}$, I. Burmeister ${ }^{43}$, E. Busato ${ }^{34}$, D. Büscher ${ }^{48}$, V. Büscher ${ }^{82}$, P. Bussey ${ }^{53}$, C.P. Buszello ${ }^{167}$, B. Butler ${ }^{57}$, J.M. Butler ${ }^{22}$, A.I. Butt ${ }^{3}$, C.M. Buttar ${ }^{53}$, J.M. Butterworth ${ }^{77}$, P. Butti ${ }^{106}$, W. Buttinger ${ }^{28}$, A. Buzatu ${ }^{53}$, M. Byszewski ${ }^{10}$,

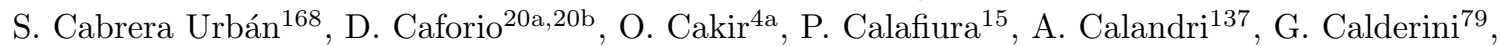
P. Calfayan ${ }^{99}$, R. Calkins ${ }^{107}$, L.P. Caloba ${ }^{24 a}$, D. Calvet ${ }^{34}$, S. Calvet $^{34}$, R. Camacho Toro ${ }^{49}$, S. Camarda ${ }^{42}$, D. Cameron ${ }^{118}$, L.M. Caminada ${ }^{15}$, R. Caminal Armadans ${ }^{12}$, S. Campana ${ }^{30}$,

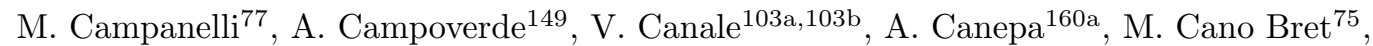
J. Cantero ${ }^{81}$, R. Cantrill ${ }^{125 a}$, T. Cao ${ }^{40}$, M.D.M. Capeans Garrido ${ }^{30}$, I. Caprini ${ }^{26 a}$, M. Caprini ${ }^{26 a}$, M. Capua ${ }^{37 a, 37 b}$, R. Caputo ${ }^{82}$, R. Cardarelli ${ }^{134 a}$, T. Carli ${ }^{30}$, G. Carlino ${ }^{103 a}$, L. Carminati ${ }^{90 a, 90 b}$, S. Caron ${ }^{105}$, E. Carquin ${ }^{32 a}$, G.D. Carrillo-Montoya ${ }^{146 c}$, J.R. Carter ${ }^{28}$, J. Carvalho ${ }^{125 a, 125 c}$, D. Casadei ${ }^{77}$, M.P. Casado ${ }^{12}$, M. Casolino ${ }^{12}$, E. Castaneda-Miranda ${ }^{146 b}$, A. Castelli ${ }^{106}$, V. Castillo Gimenez ${ }^{168}$, N.F. Castro ${ }^{125 a}$, P. Catastini ${ }^{57}$, A. Catinaccio ${ }^{30}$, J.R. Catmore ${ }^{118}$, A. Cattai ${ }^{30}$, G. Cattani ${ }^{134 a, 134 b}$, S. Caughron ${ }^{89}$, V. Cavaliere ${ }^{166}$, D. Cavalli ${ }^{90 a}$, M. Cavalli-Sforza ${ }^{12}$, V. Cavasinni ${ }^{123 a, 123 b}$, F. Ceradini ${ }^{135 a, 135 b}$, B. Cerio $^{45}$, K. Cerny ${ }^{128}$, A.S. Cerqueira ${ }^{24 b}$, A. Cerri ${ }^{150}$, L. Cerrito ${ }^{75}$, F. Cerutti ${ }^{15}$, M. Cerv ${ }^{30}$, A. Cervelli ${ }^{17}$, S.A. Cetin ${ }^{19 b}$, A. Chafaq ${ }^{136 a}$, D. Chakraborty ${ }^{107}$, I. Chalupkova ${ }^{128}$, P. Chang $^{166}$, B. Chapleau ${ }^{86}$, J.D. Chapman ${ }^{28}$, D. Charfeddine ${ }^{116}$, D.G. Charlton ${ }^{18}$, C.C. Chau ${ }^{159}$, C.A. Chavez Barajas ${ }^{150}$, S. Cheatham ${ }^{86}$, A. Chegwidden ${ }^{89}$, S. Chekanov ${ }^{6}$, S.V. Chekulaev ${ }^{160 a}$, G.A. Chelkov ${ }^{64, f}$, M.A. Chelstowska ${ }^{88}$, C. Chen ${ }^{63}$, H. Chen ${ }^{25}$, K. Chen ${ }^{149}$, L. Chen ${ }^{33 d, g}$, S. Chen ${ }^{33 c}$, X. Chen ${ }^{146 c}$, Y. Chen ${ }^{66}$, Y. Chen ${ }^{35}$, H.C. Cheng ${ }^{88}$, Y. Cheng ${ }^{31}$, A. Cheplakov ${ }^{64}$, R. Cherkaoui El Moursli136e, V. Chernyatin ${ }^{25, *}$, E. Cheu ${ }^{7}$, L. Chevalier ${ }^{137}$, V. Chiarella ${ }^{47}$, G. Chiefari103a,103b , J.T. Childers ${ }^{6}$,

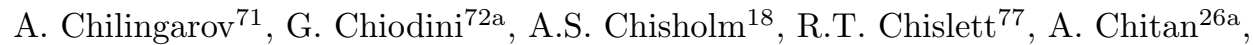
M.V. Chizhov ${ }^{64}$, S. Chouridou ${ }^{9}$, B.K.B. Chow $^{99}$, D. Chromek-Burckhart ${ }^{30}$, M.L. Chu ${ }^{152}$, J. Chudoba ${ }^{126}$, J.J. Chwastowski ${ }^{39}$, L. Chytka ${ }^{114}$, G. Ciapetti ${ }^{133 a, 133 b}$, A.K. Ciftci ${ }^{4 a}$, R. Ciftci ${ }^{4 a}$, D. Cinca ${ }^{53}$, V. Cindro ${ }^{74}$, A. Ciocio ${ }^{15}$, P. Cirkovic $^{13 b}$, Z.H. Citron ${ }^{173}$, M. Citterio ${ }^{90 a}$, M. Ciubancan ${ }^{26 a}$, A. Clark ${ }^{49}$, P.J. Clark ${ }^{46}$, R.N. Clarke ${ }^{15}$, W. Cleland ${ }^{124}$, J.C. Clemens ${ }^{84}$, C. Clement ${ }^{147 a, 147 b}$, Y. Coadou ${ }^{84}$, M. Cobal ${ }^{165 a, 165 c}$, A. Coccaro ${ }^{139}$, J. Cochran ${ }^{63}$, L. Coffey ${ }^{23}$, J.G. Cogan ${ }^{144}$, J. Coggeshall ${ }^{166}$, B. Cole ${ }^{35}$, S. Cole ${ }^{107}$, A.P. Colijn ${ }^{106}$, J. Collot ${ }^{55}$, T. Colombo ${ }^{58 c}$, G. Colon ${ }^{85}$, G. Compostella ${ }^{100}$, P. Conde Muiño ${ }^{125 a, 125 b}$, E. Coniavitis ${ }^{48}$, M.C. Conidi ${ }^{12}$, S.H. Connell ${ }^{146 b}$, I.A. Connelly ${ }^{76}$, S.M. Consonni ${ }^{90 a, 90 b}$, V. Consorti ${ }^{48}$, S. Constantinescu ${ }^{26 a}$, C. Conta ${ }^{120 a, 120 b}$, G. Conti ${ }^{57}$, F. Conventi ${ }^{103 a}, h$, M. Cooke ${ }^{15}$, B.D. Cooper ${ }^{77}$, A.M. Cooper-Sarkar ${ }^{119}$, N.J. Cooper-Smith ${ }^{76}$, K. Copic ${ }^{15}$, T. Cornelissen ${ }^{176}$, M. Corradi ${ }^{20 a}$, 
F. Corriveau ${ }^{86, i}$, A. Corso-Radu ${ }^{164}$, A. Cortes-Gonzalez ${ }^{12}$, G. Cortiana ${ }^{100}$, G. Costa $^{90 a}$, M.J. Costa ${ }^{168}$, D. Costanzo ${ }^{140}$, D. Côté ${ }^{8}$, G. Cottin $^{28}$, G. Cowan ${ }^{76}$, B.E. Cox ${ }^{83}$, K. Cranmer ${ }^{109}$, G. Cree ${ }^{29}$, S. Crépé-Renaudin ${ }^{55}$, F. Crescioli ${ }^{79}$, W.A. Cribbs ${ }^{147 a, 147 b}$, M. Crispin Ortuzar ${ }^{119}$, M. Cristinziani ${ }^{21}$, V. Croft ${ }^{105}$, G. Crosetti ${ }^{37 a, 37 b}$, C.-M. Cuciuc ${ }^{26 a}$, T. Cuhadar Donszelmann ${ }^{140}$, J. Cummings ${ }^{177}$, M. Curatolo ${ }^{47}$, C. Cuthbert ${ }^{151}$, H. Czirr ${ }^{142}$, P. Czodrowski ${ }^{3}$, Z. Czyczula ${ }^{177}$, S. D’Auria ${ }^{53}$, M. D'Onofrio ${ }^{73}$, M.J. Da Cunha Sargedas De Sousa ${ }^{125 a, 125 b}$, C. Da Via ${ }^{83}$, W. Dabrowski ${ }^{38 a}$, A. Dafinca ${ }^{119}$, T. Dai ${ }^{88}$, O. Dale ${ }^{14}$, F. Dallaire ${ }^{94}$, C. Dallapiccola ${ }^{85}$, M. Dam $^{36}$, A.C. Daniells ${ }^{18}$, M. Dano Hoffmann ${ }^{137}$, V. Dao ${ }^{48}$, G. Darbo ${ }^{50 a}$, S. Darmora ${ }^{8}$, J.A. Dassoulas ${ }^{42}$, A. Dattagupta ${ }^{60}$, W. Davey ${ }^{21}$, C. David ${ }^{170}$, T. Davidek ${ }^{128}$, E. Davies ${ }^{119, c}$, M. Davies ${ }^{154}$, O. Davignon ${ }^{79}$, A.R. Davison ${ }^{77}$, P. Davison ${ }^{77}$, Y. Davygora ${ }^{58 a}$, E. Dawe $^{143}$, I. Dawson ${ }^{140}$, R.K. Daya-Ishmukhametova ${ }^{85}, \mathrm{~K} . \mathrm{De}^{8}$, R. de Asmundis ${ }^{103 a}$, S. De Castro ${ }^{20 a, 20 b}$, S. De Cecco ${ }^{79}$, N. De Groot ${ }^{105}$, P. de Jong ${ }^{106}$, H. De la Torre ${ }^{81}$, F. De Lorenzi ${ }^{63}$, L. De Nooij ${ }^{106}$, D. De Pedis ${ }^{133 a}$, A. De Salvo ${ }^{133 a}$, U. De Sanctis ${ }^{165 a, 165 b}$, A. De Santo ${ }^{150}$, J.B. De Vivie De Regie ${ }^{116}$, W.J. Dearnaley ${ }^{71}$, R. Debbe ${ }^{25}$, C. Debenedetti ${ }^{138}$, B. Dechenaux ${ }^{55}$, D.V. Dedovich ${ }^{64}$, I. Deigaard ${ }^{106}$, J. Del Peso ${ }^{81}$, T. Del Prete ${ }^{123 a, 123 b}$, F. Deliot ${ }^{137}$, C.M. Delitzsch ${ }^{49}$, M. Deliyergiyev ${ }^{74}$, A. Dell'Acqua ${ }^{30}$, L. Dell'Asta ${ }^{22}$, M. Dell'Orso ${ }^{123 a, 123 b}$, M. Della Pietra ${ }^{103 a, h}$, D. della Volpe ${ }^{49}$, M. Delmastro ${ }^{5}$, P.A. Delsart ${ }^{55}$, C. Deluca ${ }^{106}$, S. Demers ${ }^{177}$, M. Demichev ${ }^{64}$, A. Demilly ${ }^{79}$, S.P. Denisov ${ }^{129}$, D. Derendarz ${ }^{39}$, J.E. Derkaoui ${ }^{136 d}$, F. Derue ${ }^{79}$, P. Dervan ${ }^{73}$, K. Desch $^{21}$, C. Deterre ${ }^{42}$, P.O. Deviveiros ${ }^{106}$, A. Dewhurst ${ }^{130}$, S. Dhaliwal ${ }^{106}$,

A. Di Ciaccio134a,134b , L. Di Ciaccio ${ }^{5}$, A. Di Domenico ${ }^{133 a, 133 b}$, C. Di Donato ${ }^{103 a, 103 b}$, A. Di Girolamo ${ }^{30}$, B. Di Girolamo ${ }^{30}$, A. Di Mattia ${ }^{153}$, B. Di Micco ${ }^{135 a, 135 b}$, R. Di Nardo ${ }^{47}$,

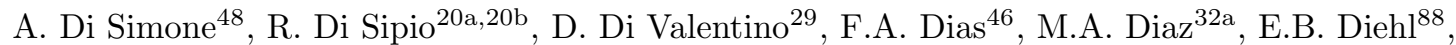
J. Dietrich ${ }^{42}$, T.A. Dietzsch ${ }^{58 a}$, S. Diglio ${ }^{84}$, A. Dimitrievska ${ }^{13 a}$, J. Dingfelder $^{21}$,

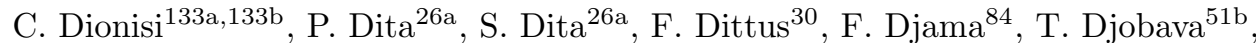
M.A.B. do Vale ${ }^{24 c}$, A. Do Valle Wemans ${ }^{125 a, 125 g}$, T.K.O. Doan ${ }^{5}$, D. Dobos ${ }^{30}$, C. Doglioni ${ }^{49}$, T. Doherty ${ }^{53}$, T. Dohmae ${ }^{156}$, J. Dolejsi ${ }^{128}$, Z. Dolezal ${ }^{128}$, B.A. Dolgoshein ${ }^{97, *}$, M. Donadelli ${ }^{24 d}$, S. Donati ${ }^{123 a, 123 b}$, P. Dondero ${ }^{120 a, 120 b}$, J. Donini $^{34}$, J. Dopke ${ }^{130}$, A. Doria ${ }^{103 a}$, M.T. Dova ${ }^{70}$, A.T. Doyle ${ }^{53}$, M. Dris ${ }^{10}$, J. Dubbert ${ }^{88}$, S. Dube ${ }^{15}$, E. Dubreuil ${ }^{34}$, E. Duchovni ${ }^{173}$, G. Duckeck ${ }^{99}$,

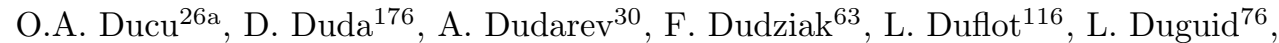
M. Dührssen ${ }^{30}$, M. Dunford ${ }^{58 a}$, H. Duran Yildiz ${ }^{4 a}$, M. Düren ${ }^{52}$, A. Durglishvili ${ }^{51 b}$, M. Dwuznik ${ }^{38 a}$, M. Dyndal ${ }^{38 a}$, J. Ebke ${ }^{99}$, W. Edson ${ }^{2}$, N.C. Edwards ${ }^{46}$, W. Ehrenfeld ${ }^{21}$, T. Eifert ${ }^{144}$, G. Eigen ${ }^{14}$, K. Einsweiler ${ }^{15}$, T. Ekelof ${ }^{167}$, M. El Kacimi ${ }^{136 c}$, M. Ellert ${ }^{167}$, S. Elles ${ }^{5}$, F. Ellinghaus ${ }^{82}$, N. Ellis ${ }^{30}$, J. Elmsheuser ${ }^{99}$, M. Elsing ${ }^{30}$, D. Emeliyanov ${ }^{130}$, Y. Enari ${ }^{156}$, O.C. Endner ${ }^{82}$, M. Endo ${ }^{117}$, R. Engelmann ${ }^{149}$, J. Erdmann ${ }^{177}$, A. Ereditato ${ }^{17}$, D. Eriksson ${ }^{147 a}$, G. Ernis ${ }^{176}$, J. Ernst ${ }^{2}$, M. Ernst ${ }^{25}$, J. Ernwein ${ }^{137}$, D. Errede ${ }^{166}$, S. Errede ${ }^{166}$, E. Ertel ${ }^{82}$, M. Escalier ${ }^{116}$, H. Esch $^{43}$, C. Escobar ${ }^{124}$, B. Esposito ${ }^{47}$, A.I. Etienvre ${ }^{137}$, E. Etzion ${ }^{154}$, H. Evans ${ }^{60}$, A. Ezhilov ${ }^{122}$, L. Fabbri ${ }^{20 a, 20 b}$, G. Facini ${ }^{31}$, R.M. Fakhrutdinov ${ }^{129}$, S. Falciano ${ }^{133 a}$, R.J. Falla ${ }^{77}$, J. Faltova ${ }^{128}$, Y. Fang ${ }^{33 a}$, M. Fanti ${ }^{90 a, 90 b}$, A. Farbin ${ }^{8}$, A. Farilla ${ }^{135 a}$, T. Farooque ${ }^{12}$, S. Farrell ${ }^{15}$, S.M. Farrington ${ }^{171}$, P. Farthouat ${ }^{30}$, F. Fassi ${ }^{136 e}$, P. Fassnacht ${ }^{30}$, D. Fassouliotis ${ }^{9}$, A. Favareto ${ }^{50 a, 50 b}$, L. Fayard ${ }^{116}$, P. Federic ${ }^{145 a}$, O.L. Fedin ${ }^{122, j}$, W. Fedorko ${ }^{169}$, M. Fehling-Kaschek ${ }^{48}$, S. Feigl ${ }^{30}$, L. Feligioni ${ }^{84}$, C. Feng ${ }^{33 d}$, E.J. Feng ${ }^{6}$, H. Feng ${ }^{88}$, A.B. Fenyuk ${ }^{129}$, S. Fernandez Perez $^{30}$, S. Ferrag ${ }^{53}$, J. Ferrando ${ }^{53}$, A. Ferrari ${ }^{167}$, P. Ferrari ${ }^{106}$, R. Ferrari ${ }^{120 a}$, D.E. Ferreira de Lima $^{53}$, A. Ferrer ${ }^{168}$, D. Ferrere ${ }^{49}$, C. Ferretti ${ }^{88}$,

A. Ferretto Parodi ${ }^{50 a, 50 b}$, M. Fiascaris ${ }^{31}$, F. Fiedler ${ }^{82}$, A. Filipčič ${ }^{74}$, M. Filipuzzi ${ }^{42}$, F. Filthaut ${ }^{105}$, M. Fincke-Keeler ${ }^{170}$, K.D. Finelli ${ }^{151}$, M.C.N. Fiolhais ${ }^{125 a, 125 c}$, L. Fiorini ${ }^{168}$, A. Firan ${ }^{40}$,

A. Fischer ${ }^{2}$, J. Fischer ${ }^{176}$, W.C. Fisher ${ }^{89}$, E.A. Fitzgerald ${ }^{23}$, M. Flechl ${ }^{48}$, I. Fleck ${ }^{142}$, P. Fleischmann ${ }^{88}$, S. Fleischmann ${ }^{176}$, G.T. Fletcher ${ }^{140}$, G. Fletcher ${ }^{75}$, T. Flick ${ }^{176}$, A. Floderus ${ }^{80}$, L.R. Flores Castillo ${ }^{174, k}$, A.C. Florez Bustos ${ }^{160 b}$, M.J. Flowerdew ${ }^{100}$, A. Formica ${ }^{137}$, A. Forti ${ }^{83}$, 
D. Fortin ${ }^{160 a}$, D. Fournier ${ }^{116}$, H. Fox ${ }^{71}$, S. Fracchia ${ }^{12}$, P. Francavilla ${ }^{79}$, M. Franchini20a,20b, S. Franchino ${ }^{30}$, D. Francis ${ }^{30}$, L. Franconi ${ }^{118}$, M. Franklin ${ }^{57}$, S. Franz ${ }^{61}$, M. Fraternali ${ }^{120 a, 120 b}$, S.T. French ${ }^{28}$, C. Friedrich ${ }^{42}$, F. Friedrich ${ }^{44}$, D. Froidevaux ${ }^{30}$, J.A. Frost ${ }^{28}$, C. Fukunaga ${ }^{157}$, E. Fullana Torregrosa ${ }^{82}$, B.G. Fulsom ${ }^{144}$, J. Fuster ${ }^{168}$, C. Gabaldon ${ }^{55}$, O. Gabizon ${ }^{173}$, A. Gabrielli ${ }^{20 a, 20 b}$, A. Gabrielli ${ }^{133 a, 133 b}$, S. Gadatsch ${ }^{106}$, S. Gadomski $^{49}$, G. Gagliardi ${ }^{50 a, 50 b}$, P. Gagnon ${ }^{60}$, C. Galea ${ }^{105}$, B. Galhardo ${ }^{125 a, 125 c}$, E.J. Gallas ${ }^{119}$, V. Gallo ${ }^{17}$, B.J. Gallop ${ }^{130}$, P. Gallus ${ }^{127}$, G. Galster ${ }^{36}$, K.K. Gan ${ }^{110}$, J. Gao ${ }^{33 b, g}$, Y.S. Gao ${ }^{144, e}$, F.M. Garay Walls ${ }^{46}$, F. Garberson ${ }^{177}$, C. García ${ }^{168}$, J.E. García Navarro ${ }^{168}$, M. Garcia-Sciveres ${ }^{15}$, R.W. Gardner ${ }^{31}$, N. Garelli ${ }^{144}$, V. Garonne ${ }^{30}$, C. Gatti ${ }^{47}$, G. Gaudio ${ }^{120 a}$, B. Gaur ${ }^{142}$, L. Gauthier ${ }^{94}$, P. Gauzzi ${ }^{133 a, 133 b}$, I.L. Gavrilenko ${ }^{95}$, C. Gay ${ }^{169}$, G. Gaycken ${ }^{21}$, E.N. Gazis ${ }^{10}$, P. Ge ${ }^{33 d}$, Z. Gecse ${ }^{169}$, C.N.P. Gee ${ }^{130}$, D.A.A. Geerts ${ }^{106}$, Ch. Geich-Gimbel ${ }^{21}$, K. Gellerstedt ${ }^{147 a, 147 b}$, C. Gemme ${ }^{50 a}$, A. Gemmell ${ }^{53}$, M.H. Genest ${ }^{55}$, S. Gentile ${ }^{133 a, 133 b}$, M. George ${ }^{54}$, S. George ${ }^{76}$, D. Gerbaudo ${ }^{164}$, A. Gershon ${ }^{154}$, H. Ghazlane ${ }^{136 b}$, N. Ghodbane ${ }^{34}$, B. Giacobbe ${ }^{20 a}$, S. Giagu ${ }^{133 a, 133 b}$, V. Giangiobbe ${ }^{12}$, P. Giannetti ${ }^{123 a, 123 b}$, F. Gianotti ${ }^{30}$, B. Gibbard ${ }^{25}$, S.M. Gibson ${ }^{76}$, M. Gilchriese ${ }^{15}$, T.P.S. Gillam ${ }^{28}$, D. Gillberg ${ }^{30}$, G. Gilles ${ }^{34}$, D.M. Gingrich ${ }^{3, d}$, N. Giokaris ${ }^{9}$, M.P. Giordani ${ }^{165 a, 165 c}$, R. Giordano ${ }^{103 a, 103 b}$, F.M. Giorgi ${ }^{20 a}$, F.M. Giorgi ${ }^{16}$, P.F. Giraud ${ }^{137}$, D. Giugni ${ }^{90 a}$, C. Giuliani ${ }^{48}$, M. Giulini ${ }^{58 b}$, B.K. Gjelsten ${ }^{118}$, S. Gkaitatzis ${ }^{155}$, I. Gkialas ${ }^{155, l}$, L.K. Gladilin ${ }^{98}$, C. Glasman ${ }^{81}$, J. Glatzer ${ }^{30}$, P.C.F. Glaysher ${ }^{46}$, A. Glazov ${ }^{42}$, G.L. Glonti ${ }^{64}$, M. Goblirsch-Kolb ${ }^{100}$, J.R. Goddard ${ }^{75}$, J. Godfrey ${ }^{143}$, J. Godlewski ${ }^{30}$, C. Goeringer ${ }^{82}$, S. Goldfarb ${ }^{88}$, T. Golling ${ }^{177}$, D. Golubkov ${ }^{129}$, A. Gomes ${ }^{125 a, 125 b, 125 d}$, L.S. Gomez Fajardo ${ }^{42}$, R. Gonçalo ${ }^{125 a}$, J. Goncalves Pinto Firmino Da Costa ${ }^{137}$, L. Gonella ${ }^{21}$, S. González de la Hoz ${ }^{168}$, G. Gonzalez Parra ${ }^{12}$, S. Gonzalez-Sevilla ${ }^{49}$, L. Goossens ${ }^{30}$, P.A. Gorbounov ${ }^{96}$, H.A. Gordon ${ }^{25}$, I. Gorelov ${ }^{104}$, B. Gorini ${ }^{30}$, E. Gorini ${ }^{72 a}{ }^{72 b}$, A. Gorišek ${ }^{74}$, E. Gornicki ${ }^{39}$, A.T. Goshaw ${ }^{6}$, C. Gössling ${ }^{43}$, M.I. Gostkin ${ }^{64}$, M. Gouighri136a ${ }^{13}$ D. Goujdami ${ }^{136 c}$, M.P. Goulette ${ }^{49}$, A.G. Goussiou ${ }^{139}$, C. Goy ${ }^{5}$, S. Gozpinar ${ }^{23}$, H.M.X. Grabas ${ }^{137}$, L. Graber ${ }^{54}$, I. Grabowska-Bold ${ }^{38 a}$, P. Grafström ${ }^{20 a, 20 b}$, K-J. Grahn ${ }^{42}$, J. Gramling ${ }^{49}$, E. Gramstad ${ }^{118}$, S. Grancagnolo ${ }^{16}$, V. Grassi ${ }^{149}$, V. Gratchev ${ }^{122}$, H.M. Gray ${ }^{30}$, E. Graziani ${ }^{135 a}$, O.G. Grebenyuk ${ }^{122}$, Z.D. Greenwood ${ }^{78, m}$, K. Gregersen ${ }^{77}$, I.M. Gregor ${ }^{42}$, P. Grenier ${ }^{144}$, J. Griffiths ${ }^{8}$, A.A. Grillo ${ }^{138}$, K. Grimm ${ }^{71}$, S. Grinstein ${ }^{12, n}$, Ph. Gris ${ }^{34}$, Y.V. Grishkevich ${ }^{98}$, J.-F. Grivaz ${ }^{116}$, J.P. Grohs ${ }^{44}$, A. Grohsjean ${ }^{42}$, E. Gross ${ }^{173}$, J. Grosse-Knetter ${ }^{54}$, G.C. Grossi ${ }^{134 a, 134 b}$, J. Groth-Jensen ${ }^{173}$, Z.J. Grout ${ }^{150}$, L. Guan ${ }^{33 b}$, F. Guescini ${ }^{49}$, D. Guest ${ }^{177}$, O. Gueta ${ }^{154}$, C. Guicheney ${ }^{34}$, E. Guido50a,50b, T. Guillemin ${ }^{116}$, S. Guindon ${ }^{2}$, U. Gul ${ }^{53}$, C. Gumpert ${ }^{44}$, J. Gunther ${ }^{127}$, J. Guo ${ }^{35}$, S. Gupta ${ }^{119}$, P. Gutierrez ${ }^{112}$, N.G. Gutierrez Ortiz ${ }^{53}$, C. Gutschow ${ }^{77}$, N. Guttman ${ }^{154}$, C. Guyot ${ }^{137}$, C. Gwenlan ${ }^{119}$, C.B. Gwilliam ${ }^{73}$, A. Haas ${ }^{109}$, C. Haber ${ }^{15}$, H.K. Hadavand ${ }^{8}$, N. Haddad ${ }^{136 e}$, P. Haefner ${ }^{21}$, S. Hageböck ${ }^{21}$, Z. Hajduk ${ }^{39}$, H. Hakobyan ${ }^{178}$, M. Haleem ${ }^{42}$, D. Hall ${ }^{119}$, G. Halladjian ${ }^{89}$, K. Hamacher ${ }^{176}$, P. Hamal ${ }^{114}$, K. Hamano ${ }^{170}$, M. Hamer ${ }^{54}$, A. Hamilton ${ }^{146 a}$, S. Hamilton ${ }^{162}$, G.N. Hamity ${ }^{146 c}$, P.G. Hamnett ${ }^{42}$, L. Han ${ }^{33 b}$, K. Hanagaki ${ }^{117}$, K. Hanawa ${ }^{156}$, M. Hance ${ }^{15}$, P. Hanke ${ }^{58 a}$, R. Hanna ${ }^{137}$, J.B. Hansen ${ }^{36}$,

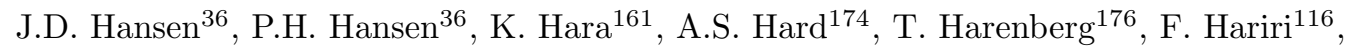
S. Harkusha ${ }^{91}$, D. Harper ${ }^{88}$, R.D. Harrington ${ }^{46}$, O.M. Harris ${ }^{139}$, P.F. Harrison ${ }^{171}$, F. Hartjes ${ }^{106}$, M. Hasegawa ${ }^{66}$, S. Hasegawa ${ }^{102}$, Y. Hasegawa ${ }^{141}$, A. Hasib ${ }^{112}$, S. Hassani ${ }^{137}$, S. Haug ${ }^{17}$, M. Hauschild ${ }^{30}$, R. Hauser ${ }^{89}$, M. Havranek ${ }^{126}$, C.M. Hawkes ${ }^{18}$, R.J. Hawkings ${ }^{30}$, A.D. Hawkins ${ }^{80}$,

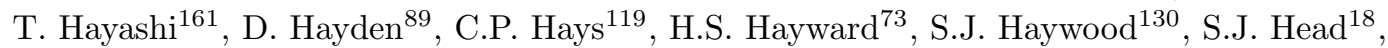
T. Heck ${ }^{82}$, V. Hedberg ${ }^{80}$, L. Heelan ${ }^{8}$, S. Heim ${ }^{121}$, T. Heim ${ }^{176}$, B. Heinemann ${ }^{15}$, L. Heinrich ${ }^{109}$, J. Hejbal ${ }^{126}$, L. Helary ${ }^{22}$, C. Heller ${ }^{99}$, M. Heller ${ }^{30}$, S. Hellman ${ }^{147 a, 147 b}$, D. Hellmich ${ }^{21}$, C. Helsens ${ }^{30}$, J. Henderson ${ }^{119}$, R.C.W. Henderson ${ }^{71}$, Y. Heng ${ }^{174}$, C. Hengler ${ }^{42}$, A. Henrichs ${ }^{177}$, A.M. Henriques Correia ${ }^{30}$, S. Henrot-Versille ${ }^{116}$, C. Hensel ${ }^{54}$, G.H. Herbert ${ }^{16}$, Y. Hernández Jiménez ${ }^{168}$, R. Herrberg-Schubert ${ }^{16}$, G. Herten ${ }^{48}$, R. Hertenberger ${ }^{99}$, L. Hervas ${ }^{30}$, 
G.G. Hesketh ${ }^{77}$, N.P. Hessey ${ }^{106}$, R. Hickling ${ }^{75}$, E. Higón-Rodriguez ${ }^{168}$, E. Hill ${ }^{170}$, J.C. Hill ${ }^{28}$, K.H. Hiller ${ }^{42}$, S. Hillert ${ }^{21}$, S.J. Hillier ${ }^{18}$, I. Hinchliffe ${ }^{15}$, E. Hines ${ }^{121}$, M. Hirose ${ }^{158}$, D. Hirschbuehl ${ }^{176}$, J. Hobbs ${ }^{149}$, N. Hod ${ }^{106}$, M.C. Hodgkinson ${ }^{140}$, P. Hodgson ${ }^{140}$, A. Hoecker ${ }^{30}$, M.R. Hoeferkamp ${ }^{104}$, F. Hoenig ${ }^{99}$, J. Hoffman ${ }^{40}$, D. Hoffmann ${ }^{84}$, J.I. Hofmann ${ }^{58 a}$, M. Hohlfeld ${ }^{82}$, T.R. Holmes ${ }^{15}$, T.M. Hong ${ }^{121}$, L. Hooft van Huysduynen ${ }^{109}$, Y. Horii ${ }^{102}$, J-Y. Hostachy ${ }^{55}$, S. Hou ${ }^{152}$, A. Hoummada ${ }^{136 a}$, J. Howard ${ }^{119}$, J. Howarth ${ }^{42}$, M. Hrabovsky ${ }^{114}$, I. Hristova ${ }^{16}$, J. Hrivnac ${ }^{116}$, T. Hryn'ova ${ }^{5}$, C. $\mathrm{Hsu}^{146 c}$, P.J. $\mathrm{Hsu}^{82}$, S.-C. $\mathrm{Hsu}^{139}$, D. $\mathrm{Hu}^{35}$, X. Hu${ }^{25}$, Y. Huang ${ }^{42}$, Z. Hubacek ${ }^{30}$, F. Hubaut ${ }^{84}$, F. Huegging ${ }^{21}$, T.B. Huffman ${ }^{119}$, E.W. Hughes ${ }^{35}$, G. Hughes ${ }^{71}$,

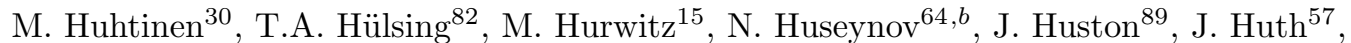

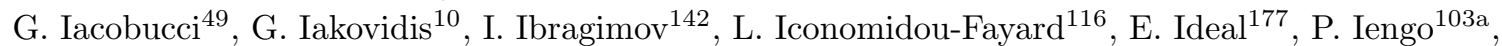
O. Igonkina ${ }^{106}$, T. Iizawa ${ }^{172}$, Y. Ikegami ${ }^{65}$, K. Ikematsu ${ }^{142}$, M. Ikeno ${ }^{65}$, Y. Ilchenko ${ }^{31, o}$,

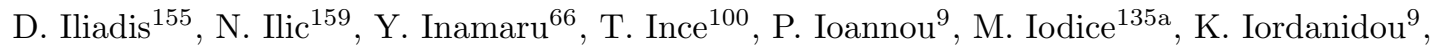
V. Ippolito ${ }^{57}$, A. Irles Quiles ${ }^{168}$, C. Isaksson ${ }^{167}$, M. Ishino ${ }^{67}$, M. Ishitsuka ${ }^{158}$, R. Ishmukhametov ${ }^{110}$, C. Issever ${ }^{119}$, S. Istin ${ }^{19 a}$, J.M. Iturbe Ponce ${ }^{83}$, R. Iuppa ${ }^{134 a, 134 b}$, J. Ivarsson ${ }^{80}$, W. Iwanski ${ }^{39}$, H. Iwasaki ${ }^{65}$, J.M. Izen ${ }^{41}$, V. Izzo ${ }^{103 a}$, B. Jackson ${ }^{121}$, M. Jackson ${ }^{73}$, P. Jackson ${ }^{1}$, M.R. Jaekel ${ }^{30}$, V. Jain ${ }^{2}$, K. Jakobs ${ }^{48}$, S. Jakobsen ${ }^{30}$, T. Jakoubek ${ }^{126}$, J. Jakubek ${ }^{127}$, D.O. Jamin ${ }^{152}$, D.K. Jana ${ }^{78}$, E. Jansen ${ }^{77}$, H. Jansen ${ }^{30}$, J. Janssen ${ }^{21}$, M. Janus ${ }^{171}$, G. Jarlskog ${ }^{80}$, N. Javadov ${ }^{64, b}$, T. Javůrek ${ }^{48}$, L. Jeanty ${ }^{15}$, J. Jejelava ${ }^{51 a, p}$, G.-Y. Jeng ${ }^{151}$, D. Jennens ${ }^{87}$, P. Jenni ${ }^{48, q}$, J. Jentzsch ${ }^{43}$, C. Jeske ${ }^{171}$, S. Jézéquel ${ }^{5}$, H. Ji ${ }^{174}$, J. Jia ${ }^{149}$, Y. Jiang ${ }^{33 b}$, M. Jimenez Belenguer ${ }^{42}$, S. Jin ${ }^{33 a}$, A. Jinaru ${ }^{26 a}$, O. Jinnouchi ${ }^{158}$, M.D. Joergensen ${ }^{36}$, K.E. Johansson ${ }^{147 a, 147 b}$, P. Johansson ${ }^{140}$, K.A. Johns ${ }^{7}$, K. Jon-And ${ }^{147 a, 147 b}$, G. Jones ${ }^{171}$, R.W.L. Jones ${ }^{71}$, T.J. Jones ${ }^{73}$, J. Jongmanns ${ }^{58 a}$, P.M. Jorge ${ }^{125 a, 125 b}$, K.D. Joshi ${ }^{83}$, J. Jovicevic ${ }^{148}$, X. Ju ${ }^{174}$, C.A. Jung ${ }^{43}$, R.M. Jungst ${ }^{30}$, P. Jussel ${ }^{61}$, A. Juste Rozas ${ }^{12, n}$, M. Kaci ${ }^{168}$, A. Kaczmarska ${ }^{39}$, M. Kado ${ }^{116}$, H. Kagan ${ }^{110}$, M. Kagan ${ }^{144}$, E. Kajomovitz ${ }^{45}$, C.W. Kalderon ${ }^{119}$, S. Kama ${ }^{40}$, A. Kamenshchikov ${ }^{129}$, N. Kanaya ${ }^{156}$, M. Kaneda ${ }^{30}$, S. Kaneti ${ }^{28}$, V.A. Kantserov ${ }^{97}$, J. Kanzaki ${ }^{65}$, B. Kaplan ${ }^{109}$, A. Kapliy ${ }^{31}$, D. Kar ${ }^{53}$, K. Karakostas ${ }^{10}$, N. Karastathis ${ }^{10}$, M. Karnevskiy ${ }^{82}$, S.N. Karpov ${ }^{64}$, Z.M. Karpova ${ }^{64}$, K. Karthik ${ }^{109}$, V. Kartvelishvili ${ }^{71}$, A.N. Karyukhin ${ }^{129}$, L. Kashif ${ }^{174}$, G. Kasieczka ${ }^{58 b}$, R.D. Kass ${ }^{110}$, A. Kastanas ${ }^{14}$, Y. Kataoka ${ }^{156}$, A. Katre ${ }^{49}$, J. Katzy ${ }^{42}$, V. Kaushik ${ }^{7}$, K. Kawagoe ${ }^{69}$, T. Kawamoto ${ }^{156}$, G. Kawamura ${ }^{54}$, S. Kazama ${ }^{156}$, V.F. Kazanin ${ }^{108}$, M.Y. Kazarinov ${ }^{64}$, R. Keeler ${ }^{170}$, R. Kehoe ${ }^{40}$, M. Keil ${ }^{54}$, J.S. Keller ${ }^{42}$, J.J. Kempster ${ }^{76}$, H. Keoshkerian ${ }^{5}$, O. Kepka ${ }^{126}$, B.P. Kerševan ${ }^{74}$, S. Kersten ${ }^{176}$, K. Kessoku ${ }^{156}$, J. Keung ${ }^{159}$, F. Khalil-zada ${ }^{11}$, H. Khandanyan ${ }^{147 a, 147 b}$, A. Khanov ${ }^{113}$, A. Khodinov ${ }^{97}$, A. Khomich ${ }^{58 a}$, T.J. Khoo ${ }^{28}$, G. Khoriauli ${ }^{21}$, A. Khoroshilov ${ }^{176}$, V. Khovanskiy ${ }^{96}$, E. Khramov ${ }^{64}$, J. Khubua ${ }^{51 b}$, H.Y. Kim ${ }^{8}$, H. Kim ${ }^{147 a, 147 b}$, S.H. Kim ${ }^{161}$, N. Kimura ${ }^{172}$, O. Kind ${ }^{16}$, B.T. King ${ }^{73}$, M. King ${ }^{168}$, R.S.B. King ${ }^{119}$, S.B. King ${ }^{169}$, J. Kirk ${ }^{130}$, A.E. Kiryunin ${ }^{100}$, T. Kishimoto ${ }^{66}$, D. Kisielewska ${ }^{38 a}$, F. Kiss ${ }^{48}$, T. Kittelmann ${ }^{124}$, K. Kiuchi ${ }^{161}$, E. Kladiva ${ }^{145 b}$, M. Klein ${ }^{73}$, U. Klein ${ }^{73}$, K. Kleinknecht ${ }^{82}$, P. Klimek ${ }^{147 a, 147 b}$, A. Klimentov ${ }^{25}$, R. Klingenberg ${ }^{43}$, J.A. Klinger ${ }^{83}$, T. Klioutchnikova ${ }^{30}$, P.F. Klok ${ }^{105}$, E.-E. Kluge ${ }^{58 a}$, P. Kluit ${ }^{106}$, S. Kluth ${ }^{100}$, E. Kneringer ${ }^{61}$, E.B.F.G. Knoops ${ }^{84}$, A. Knue ${ }^{53}$, D. Kobayashi ${ }^{158}$, T. Kobayashi ${ }^{156}$, M. Kobel $^{44}$, M. Kocian ${ }^{144}$, P. Kodys ${ }^{128}$, P. Koevesarki ${ }^{21}$, T. Koffas ${ }^{29}$, E. Koffeman ${ }^{106}$, L.A. Kogan ${ }^{119}$, S. Kohlmann ${ }^{176}$, Z. Kohout ${ }^{127}$, T. Kohriki ${ }^{65}$, T. Koi ${ }^{144}$, H. Kolanoski ${ }^{16}$, I. Koletsou ${ }^{5}$, J. Koll ${ }^{89}$, A.A. Komar ${ }^{95, *}$, Y. Komori ${ }^{156}$, T. Kondo ${ }^{65}$, N. Kondrashova ${ }^{42}$, K. Köneke ${ }^{48}$, A.C. König ${ }^{105}$, S. König ${ }^{82}$, T. Kono ${ }^{65, r}$, R. Konoplich ${ }^{109, s}$, N. Konstantinidis ${ }^{77}$, R. Kopeliansky ${ }^{153}$, S. Koperny ${ }^{38 a}$, L. Köpke ${ }^{82}$, A.K. Kopp ${ }^{48}$, K. Korcyl ${ }^{39}$, K. Kordas ${ }^{155}$, A. Korn ${ }^{77}$, A.A. Korol ${ }^{108, t}$, I. Korolkov ${ }^{12}$, E.V. Korolkova ${ }^{140}$, V.A. Korotkov ${ }^{129}$, O. Kortner ${ }^{100}$, S. Kortner ${ }^{100}$, V.V. Kostyukhin ${ }^{21}$, V.M. Kotov ${ }^{64}$, A. Kotwal ${ }^{45}$, C. Kourkoumelis ${ }^{9}$, V. Kouskoura ${ }^{155}$, A. Koutsman ${ }^{160 a}$, R. Kowalewski ${ }^{170}$, T.Z. Kowalski ${ }^{38 a}$, W. Kozanecki ${ }^{137}$, A.S. Kozhin ${ }^{129}$, V. Kral ${ }^{127}$, V.A. Kramarenko ${ }^{98}$, G. Kramberger ${ }^{74}$, D. Krasnopevtsev ${ }^{97}$, M.W. Krasny ${ }^{79}$, 
A. Krasznahorkay ${ }^{30}$, J.K. Kraus ${ }^{21}$, A. Kravchenko ${ }^{25}$, S. Kreiss ${ }^{109}$, M. Kretz ${ }^{58 c}$, J. Kretzschmar ${ }^{73}$, K. Kreutzfeldt ${ }^{52}$, P. Krieger ${ }^{159}$, K. Kroeninger ${ }^{54}$, H. Kroha ${ }^{100}$, J. Kroll ${ }^{121}$, J. Kroseberg ${ }^{21}$,

J. Krstic ${ }^{13 a}$, U. Kruchonak ${ }^{64}$, H. Krüger ${ }^{21}$, T. Kruker ${ }^{17}$, N. Krumnack ${ }^{63}$, Z.V. Krumshteyn ${ }^{64}$, A. Kruse ${ }^{174}$, M.C. Kruse ${ }^{45}$, M. Kruskal ${ }^{22}$, T. Kubota ${ }^{87}$, S. Kuday ${ }^{4 a}$, S. Kuehn ${ }^{48}$, A. Kugel ${ }^{58 c}$, A. Kuhl ${ }^{138}$, T. Kuhl ${ }^{42}$, V. Kukhtin ${ }^{64}$, Y. Kulchitsky ${ }^{11}$, S. Kuleshov ${ }^{32 b}$, M. Kuna ${ }^{133 a, 133 b}$, J. Kunkle ${ }^{121}$, A. Kupco ${ }^{126}$, H. Kurashige ${ }^{66}$, Y.A. Kurochkin ${ }^{91}$, R. Kurumida ${ }^{66}$, V. Kus ${ }^{126}$, E.S. Kuwertz ${ }^{148}$, M. Kuze ${ }^{158}$, J. Kvita ${ }^{114}$, A. La Rosa ${ }^{49}$, L. La Rotonda ${ }^{37 a, 37 b}$, C. Lacasta ${ }^{168}$, F. Lacava ${ }^{133 a, 133 b}$, J. Lacey ${ }^{29}$, H. Lacker ${ }^{16}$, D. Lacour ${ }^{79}$, V.R. Lacuesta ${ }^{168}$, E. Ladygin ${ }^{64}$, R. Lafaye $^{5}$, B. Laforge ${ }^{79}$, T. Lagouri ${ }^{177}$, S. Lai ${ }^{48}$, H. Laier ${ }^{58 a}$, L. Lambourne ${ }^{77}$, S. Lammers ${ }^{60}$, C.L. Lampen ${ }^{7}$, W. Lampl ${ }^{7}$, E. Lançon ${ }^{137}$, U. Landgraf ${ }^{48}$, M.P.J. Landon ${ }^{75}$, V.S. Lang ${ }^{58 a}$,

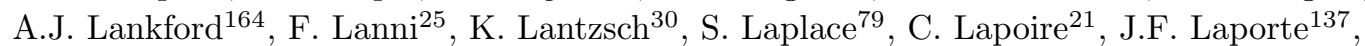

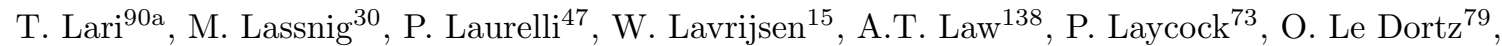
E. Le Guirriec ${ }^{84}$, E. Le Menedeu ${ }^{12}$, T. LeCompte ${ }^{6}$, F. Ledroit-Guillon ${ }^{55}$, C.A. Lee ${ }^{152}$, H. Lee ${ }^{106}$, J.S.H. Lee ${ }^{117}$, S.C. Lee ${ }^{152}$, L. Lee ${ }^{1}$, G. Lefebvre ${ }^{79}$, M. Lefebvre ${ }^{170}$, F. Legger ${ }^{99}$, C. Leggett ${ }^{15}$, A. Lehan ${ }^{73}$, M. Lehmacher ${ }^{21}$, G. Lehmann Miotto ${ }^{30}$, X. Lei ${ }^{7}$, W.A. Leight ${ }^{29}$, A. Leisos ${ }^{155}$, A.G. Leister ${ }^{177}$, M.A.L. Leite ${ }^{24 d}$, R. Leitner ${ }^{128}$, D. Lellouch ${ }^{173}$, B. Lemmer ${ }^{54}$, K.J.C. Leney ${ }^{77}$, T. Lenz ${ }^{21}$, G. Lenzen ${ }^{176}$, B. Lenzi ${ }^{30}$, R. Leone ${ }^{7}$, S. Leone ${ }^{123 a, 123 b}$, K. Leonhardt ${ }^{44}$, C. Leonidopoulos ${ }^{46}$, S. Leontsinis ${ }^{10}$, C. Leroy ${ }^{94}$, C.G. Lester ${ }^{28}$, C.M. Lester ${ }^{121}$, M. Levchenko ${ }^{122}$, J. Levêque ${ }^{5}$, D. Levin ${ }^{88}$, L.J. Levinson ${ }^{173}$, M. Levy ${ }^{18}$, A. Lewis ${ }^{119}$, G.H. Lewis ${ }^{109}$, A.M. Leyko ${ }^{21}$, M. Leyton ${ }^{41}$, B. $\mathrm{Li}^{33 \mathrm{~b}, u}$, B. $\mathrm{Li}^{84}, \mathrm{H} . \mathrm{Li}^{149}$, H.L. $\mathrm{Li}^{31}$, L. Li ${ }^{45}$, L. Li ${ }^{33 e}, \mathrm{~S} . \mathrm{Li}^{45}$, Y. $\mathrm{Li}^{33 \mathrm{c}, v}$,

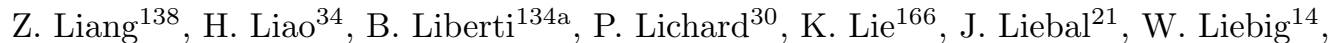
C. $\operatorname{Limbach}^{21}$, A. Limosani ${ }^{87}$, S.C. $\operatorname{Lin}^{152, w}$, T.H. $\operatorname{Lin}^{82}$, F. Linde ${ }^{106}$, B.E. Lindquist ${ }^{149}$, J.T. Linnemann ${ }^{89}$, E. Lipeles ${ }^{121}$, A. Lipniacka ${ }^{14}$, M. Lisovyi ${ }^{42}$, T.M. Liss ${ }^{166}$, D. Lissauer ${ }^{25}$, A. Lister ${ }^{169}$, A.M. Litke ${ }^{138}$, B. $\mathrm{Liu}^{152}$, D. $\mathrm{Liu}^{152}$, J.B. Liu ${ }^{33 \mathrm{~b}}$, K. Liu ${ }^{33 \mathrm{~b}, x}$, L. Liu ${ }^{88}$, M. Liu ${ }^{45}$, M. Liu ${ }^{33 \mathrm{~b}}$, Y. Liu ${ }^{33 \mathrm{~b}}$, M. Livan ${ }^{120 a, 120 b}$, S.S.A. Livermore ${ }^{119}$, A. Lleres ${ }^{55}$, J. Llorente Merino ${ }^{81}$, S.L. Lloyd ${ }^{75}$, F. Lo Sterzo ${ }^{152}$, E. Lobodzinska ${ }^{42}$, P. Loch ${ }^{7}$, W.S. Lockman ${ }^{138}$, T. Loddenkoetter ${ }^{21}$, F.K. Loebinger ${ }^{83}$, A.E. Loevschall-Jensen ${ }^{36}$, A. Loginov ${ }^{177}$, T. Lohse $^{16}$, K. Lohwasser ${ }^{42}$, M. Lokajicek ${ }^{126}$, V.P. Lombardo ${ }^{5}$, B.A. Long ${ }^{22}$, J.D. Long ${ }^{88}$, R.E. Long ${ }^{71}$, L. Lopes ${ }^{125 a}$, D. Lopez Mateos ${ }^{57}$, B. Lopez Paredes ${ }^{140}$, I. Lopez Paz ${ }^{12}$, J. Lorenz ${ }^{99}$, N. Lorenzo Martinez ${ }^{60}$,

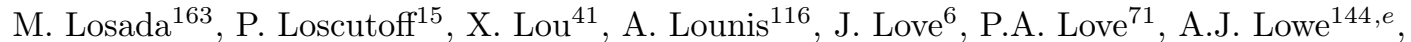
F. Lu ${ }^{33 a}$, N. Lu ${ }^{88}$, H.J. Lubatti ${ }^{139}$, C. Luci ${ }^{133 a, 133 b}$, A. Lucotte ${ }^{55}$, F. Luehring ${ }^{60}$, W. Lukas ${ }^{61}$, L. Luminari ${ }^{133 a}$, O. Lundberg ${ }^{147 a, 147 b}$, B. Lund-Jensen ${ }^{148}$, M. Lungwitz ${ }^{82}$, D. Lynn ${ }^{25}$, R. Lysak $^{126}$, E. Lytken ${ }^{80}$, H. Ma ${ }^{25}$, L.L. Ma ${ }^{33 d}$, G. Maccarrone ${ }^{47}$, A. Macchiolo ${ }^{100}$, J. Machado Miguens ${ }^{125 a, 125 b}$, D. Macina ${ }^{30}$, D. Madaffari ${ }^{84}$, R. Madar ${ }^{48}$, H.J. Maddocks ${ }^{71}$, W.F. Mader ${ }^{44}$, A. Madsen ${ }^{167}$, M. Maeno ${ }^{8}$, T. Maeno ${ }^{25}$, E. Magradze ${ }^{54}$, K. Mahboubi ${ }^{48}$, J. Mahlstedt ${ }^{106}$, S. Mahmoud ${ }^{73}$, C. Maiani ${ }^{137}$, C. Maidantchik ${ }^{24 a}$, A.A. Maier ${ }^{100}$, A. Maio ${ }^{125 a, 125 b, 125 d}$, S. Majewski ${ }^{115}$, Y. Makida ${ }^{65}$, N. Makovec ${ }^{116}$, P. Mal ${ }^{137, y}$, B. Malaescu ${ }^{79}$, Pa. Malecki ${ }^{39}$, V.P. Maleev ${ }^{122}$, F. Malek ${ }^{55}$, U. Mallik ${ }^{62}$, D. Malon ${ }^{6}$, C. Malone ${ }^{144}$, S. Maltezos ${ }^{10}$, V.M. Malyshev ${ }^{108}$, S. Malyukov ${ }^{30}$, J. Mamuzic ${ }^{13 b}$, B. Mandelli ${ }^{30}$, L. Mandelli ${ }^{90 a}$, I. Mandić ${ }^{74}$, R. Mandrysch ${ }^{62}$, J. Maneira ${ }^{125 a, 125 b}$, A. Manfredini ${ }^{100}$, L. Manhaes de Andrade Filho ${ }^{24 b}$, J.A. Manjarres Ramos ${ }^{160 b}$, A. Mann ${ }^{99}$, P.M. Manning ${ }^{138}$, A. Manousakis-Katsikakis ${ }^{9}$, B. Mansoulie ${ }^{137}$, R. Mantifel ${ }^{86}$, L. Mapelli ${ }^{30}$, L. March ${ }^{168}$, J.F. Marchand ${ }^{29}$, G. Marchiori ${ }^{79}$, M. Marcisovsky ${ }^{126}$, C.P. Marino ${ }^{170}$, M. Marjanovic ${ }^{13 a}$, C.N. Marques ${ }^{125 a}$, F. Marroquim ${ }^{24 a}$, S.P. Marsden ${ }^{83}$, Z. Marshall ${ }^{15}$, L.F. Marti ${ }^{17}$, S. Marti-Garcia ${ }^{168}$, B. Martin ${ }^{30}$, B. Martin ${ }^{89}$, T.A. Martin ${ }^{171}$, V.J. Martin ${ }^{46}$, B. Martin dit Latour ${ }^{14}$, H. Martinez ${ }^{137}$, M. Martinez ${ }^{12, n}$, S. Martin-Haugh ${ }^{130}$, A.C. Martyniuk ${ }^{77}$, M. Marx ${ }^{139}$, F. Marzano ${ }^{133 a}$, A. Marzin ${ }^{30}$, L. Masetti ${ }^{82}$, T. Mashimo ${ }^{156}$, R. Mashinistov ${ }^{95}$, J. Masik ${ }^{83}$, A.L. Maslennikov ${ }^{108}$, I. Massa ${ }^{20 a, 20 b}$, L. Massa ${ }^{20 a, 20 b}$, N. Massol ${ }^{5}$, P. Mastrandrea ${ }^{149}$, A. Mastroberardino ${ }^{37 a, 37 b}$, T. Masubuchi ${ }^{156}$, 
P. Mättig ${ }^{176}$, J. Mattmann ${ }^{82}$, J. Maurer ${ }^{26 a}$, S.J. Maxfield ${ }^{73}$, D.A. Maximov ${ }^{108, t}$, R. Mazini ${ }^{152}$, L. Mazzaferro ${ }^{134 a, 134 b}$, G. Mc Goldrick ${ }^{159}$, S.P. Mc Kee ${ }^{88}$, A. McCarn ${ }^{88}$, R.L. McCarthy ${ }^{149}$, T.G. McCarthy ${ }^{29}$, N.A. McCubbin ${ }^{130}$, K.W. McFarlane ${ }^{56, *}$, J.A. Mcfayden ${ }^{77}$, G. Mchedlidze ${ }^{54}$, S.J. McMahon ${ }^{130}$, R.A. McPherson ${ }^{170, i}$, A. Meade ${ }^{85}$, J. Mechnich ${ }^{106}$, M. Medinnis ${ }^{42}$,

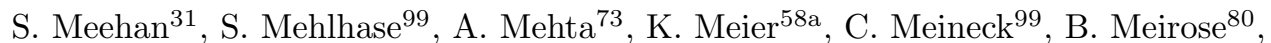
C. Melachrinos ${ }^{31}$, B.R. Mellado Garcia ${ }^{146 c}$, F. Meloni ${ }^{17}$, A. Mengarelli20a,20b, S. Menke ${ }^{100}$, E. Meoni ${ }^{162}$, K.M. Mercurio ${ }^{57}$, S. Mergelmeyer ${ }^{21}$, N. Meric ${ }^{137}$, P. Mermod ${ }^{49}$, L. Merola ${ }^{103 a, 103 b}$, C. Meroni ${ }^{90 a}$, F.S. Merritt ${ }^{31}$, H. Merritt ${ }^{110}$, A. Messina ${ }^{30, z}$, J. Metcalfe ${ }^{25}$, A.S. Mete ${ }^{164}$, C. Meyer $^{82}$, C. Meyer ${ }^{121}$, J-P. Meyer ${ }^{137}$, J. Meyer ${ }^{30}$, R.P. Middleton ${ }^{130}$, S. Migas ${ }^{73}$, L. Mijović ${ }^{21}$, G. Mikenberg ${ }^{173}$, M. Mikestikova ${ }^{126}$, M. Mikuž ${ }^{74}$, A. Milic ${ }^{30}$, D.W. Miller ${ }^{31}$, C. Mills ${ }^{46}$, A. Milov $^{173}$, D.A. Milstead ${ }^{147 a, 147 b}$, D. Milstein ${ }^{173}$, A.A. Minaenko ${ }^{129}$, I.A. Minashvili ${ }^{64}$, A.I. Mincer ${ }^{109}$, B. Mindur ${ }^{38 a}$, M. Mineev ${ }^{64}$, Y. Ming ${ }^{174}$, L.M. Mir ${ }^{12}$, G. Mirabelli133a, T. Mitani ${ }^{172}$, J. Mitrevski ${ }^{99}$, V.A. Mitsou ${ }^{168}$, S. Mitsui ${ }^{65}$, A. Miucci ${ }^{49}$, P.S. Miyagawa ${ }^{140}$, J.U. Mjörnmark ${ }^{80}$, T. Moa ${ }^{147 a, 147 b}$, K. Mochizuki ${ }^{84}$, S. Mohapatra ${ }^{35}$, W. Mohr ${ }^{48}$, S. Molander ${ }^{147 a, 147 b}$, R. Moles-Valls ${ }^{168}$, K. Mönig ${ }^{42}$, C. Monini ${ }^{55}$, J. Monk ${ }^{36}$, E. Monnier ${ }^{84}$, J. Montejo Berlingen ${ }^{12}$, F. Monticelli ${ }^{70}$, S. Monzani ${ }^{133 a, 133 b}$, R.W. Moore ${ }^{3}$, N. Morange ${ }^{62}$, D. Moreno ${ }^{82}$, M. Moreno Llácer ${ }^{54}$, P. Morettini ${ }^{50 a}$, M. Morgenstern ${ }^{44}$, M. Morii ${ }^{57}$, S. Moritz $^{82}$, A.K. Morley ${ }^{148}$, G. Mornacchi ${ }^{30}$, J.D. Morris $^{75}$, L. Morvaj102, H.G. Moser ${ }^{100}$, M. Mosidze ${ }^{51 b}$, J. Moss ${ }^{110}$, K. Motohashi ${ }^{158}$, R. Mount ${ }^{144}$, E. Mountricha ${ }^{25}$, S.V. Mouraviev ${ }^{95, *}$, E.J.W. Moyse ${ }^{85}$, S. Muanza ${ }^{84}$, R.D. Mudd ${ }^{18}$, F. Mueller ${ }^{58 a}$, J. Mueller ${ }^{124}$, K. Mueller ${ }^{21}$, T. Mueller ${ }^{28}$, T. Mueller ${ }^{82}$, D. Muenstermann ${ }^{49}$, Y. Munwes ${ }^{154}$, J.A. Murillo Quijada ${ }^{18}$, W.J. Murray ${ }^{171,130}$, H. Musheghyan ${ }^{54}$, E. Musto ${ }^{153}$, A.G. Myagkov ${ }^{129, a a}$, M. Myska ${ }^{127}$, B.P. Nachman ${ }^{144}$, O. Nackenhorst ${ }^{54}$, J. Nadal ${ }^{54}$, K. Nagai ${ }^{61}$, R. Nagai ${ }^{158}$, Y. Nagai ${ }^{84}$, K. Nagano ${ }^{65}$, A. Nagarkar ${ }^{110}$, Y. Nagasaka ${ }^{59}$, M. Nagel ${ }^{100}$, A.M. Nairz ${ }^{30}$, Y. Nakahama ${ }^{30}$, K. Nakamura ${ }^{65}$, T. Nakamura ${ }^{156}$, I. Nakano ${ }^{111}$, H. Namasivayam ${ }^{41}$, G. Nanava ${ }^{21}$, R. Narayan ${ }^{58 b}$, T. Nattermann ${ }^{21}$, T. Naumann ${ }^{42}$, G. Navarro ${ }^{163}$, R. Nayyar ${ }^{7}$, H.A. Neal ${ }^{88}$, P.Yu. Nechaeva ${ }^{95}$, T.J. Neep ${ }^{83}$, P.D. Nef ${ }^{144}$, A. Negri ${ }^{120 a, 120 b}$, G. Negri ${ }^{30}$, M. Negrini ${ }^{20 a}$, S. Nektarijevic ${ }^{49}$, A. Nelson ${ }^{164}$, T.K. Nelson ${ }^{144}$, S. Nemecek ${ }^{126}$, P. Nemethy ${ }^{109}$, A.A. Nepomuceno ${ }^{24 a}$, M. Nessi ${ }^{30, a b}{ }^{\text {, M.S. Neubauer }}{ }^{166}$, M. Neumann ${ }^{176}$, R.M. Neves ${ }^{109}$, P. Nevski ${ }^{25}$, P.R. Newman ${ }^{18}$, D.H. Nguyen ${ }^{6}$, R.B. Nickerson ${ }^{119}$, R. Nicolaidou ${ }^{137}$, B. Nicquevert ${ }^{30}$, J. Nielsen ${ }^{138}$, N. Nikiforou ${ }^{35}$, A. Nikiforov ${ }^{16}$, V. Nikolaenko ${ }^{129, a a}$, I. Nikolic-Audit ${ }^{79}$, K. Nikolics ${ }^{49}$, K. Nikolopoulos ${ }^{18}$, P. Nilsson ${ }^{8}$, Y. Ninomiya ${ }^{156}$, A. Nisati ${ }^{133 a}$, R. Nisius ${ }^{100}$, T. Nobe ${ }^{158}$, L. Nodulman ${ }^{6}$, M. Nomachi ${ }^{117}$, I. Nomidis ${ }^{29}$, S. Norberg ${ }^{112}$, M. Nordberg ${ }^{30}$, O. Novgorodova ${ }^{44}$, S. Nowak ${ }^{100}$, M. Nozaki ${ }^{65}$, L. Nozka ${ }^{114}$, K. Ntekas $^{10}$, G. Nunes Hanninger ${ }^{87}$, T. Nunnemann ${ }^{99}$, E. Nurse ${ }^{77}$, F. Nuti ${ }^{87}$, B.J. O’Brien ${ }^{46}$, F. O'grady ${ }^{7}$, D.C. O’Neil ${ }^{143}$, V. O'Shea ${ }^{53}$, F.G. Oakham ${ }^{29, d}$, H. Oberlack ${ }^{100}$,

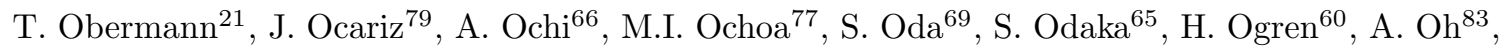
S.H. $\mathrm{Oh}^{45}$, C.C. Ohm ${ }^{15}$, H. Ohman ${ }^{167}$, W. Okamura ${ }^{117}$, H. Okawa ${ }^{25}$, Y. Okumura ${ }^{31}$, T. Okuyama ${ }^{156}$, A. Olariu ${ }^{26 a}$, A.G. Olchevski ${ }^{64}$, S.A. Olivares Pino ${ }^{46}$, D. Oliveira Damazio ${ }^{25}$, E. Oliver Garcia ${ }^{168}$, A. Olszewski ${ }^{39}$, J. Olszowska ${ }^{39}$, A. Onofre ${ }^{125 a, 125 e}$, P.U.E. Onyisi ${ }^{31, o}$, C.J. Oram ${ }^{160 a}$, M.J. Oreglia ${ }^{31}$, Y. Oren ${ }^{154}$, D. Orestano ${ }^{135 a, 135 b}$, N. Orlando ${ }^{72 a, 72 b}$, C. Oropeza Barrera ${ }^{53}$, R.S. Orr ${ }^{159}$, B. Osculati ${ }^{50 a, 50 b}$, R. Ospanov ${ }^{121}$, G. Otero y Garzon ${ }^{27}$, H. Otono ${ }^{69}$, M. Ouchrif ${ }^{136 d}$, E.A. Ouellette ${ }^{170}$, F. Ould-Saada ${ }^{118}$, A. Ouraou ${ }^{137}$, K.P. Oussoren ${ }^{106}$, Q. Ouyang ${ }^{33 a}$, A. Ovcharova ${ }^{15}$, M. Owen ${ }^{83}$, V.E. Ozcan ${ }^{19 a}$, N. Ozturk ${ }^{8}$, K. Pachal ${ }^{119}$, A. Pacheco Pages ${ }^{12}$, C. Padilla Aranda ${ }^{12}$, M. Pagáčová ${ }^{48}$, S. Pagan Griso ${ }^{15}$, E. Paganis ${ }^{140}$, C. Pahl ${ }^{100}$, F. Paige ${ }^{25}$, P. Pais ${ }^{85}$, K. Pajchel ${ }^{118}$, G. Palacino ${ }^{160 b}$, S. Palestini ${ }^{30}$, M. Palka ${ }^{38 b}$, D. Pallin ${ }^{34}$, A. Palma ${ }^{125 a, 125 b}$, J.D. Palmer ${ }^{18}$, Y.B. Pan ${ }^{174}$, E. Panagiotopoulou ${ }^{10}$, J.G. Panduro Vazquez ${ }^{76}$, P. Pani ${ }^{106}$, N. Panikashvili ${ }^{88}$, S. Panitkin ${ }^{25}$, D. Pantea ${ }^{26 a}$, L. Paolozzi ${ }^{134 a, 134 b}$, Th.D. Papadopoulou ${ }^{10}$, K. Papageorgiou ${ }^{155, l}$, A. Paramonov ${ }^{6}$, 
D. Paredes Hernandez ${ }^{34}$, M.A. Parker ${ }^{28}$, F. Parodi ${ }^{50 a, 50 b}$, J.A. Parsons ${ }^{35}$, U. Parzefall ${ }^{48}$, E. Pasqualucci ${ }^{133 a}$, S. Passaggio ${ }^{50 a}$, A. Passeri ${ }^{135 a}$, F. Pastore ${ }^{135 a, 135 b, *}$, Fr. Pastore ${ }^{76}$, G. Pásztor ${ }^{29}$, S. Pataraia ${ }^{176}$, N.D. Patel ${ }^{151}$, J.R. Pater ${ }^{83}$, S. Patricelli ${ }^{103 a, 103 b}$, T. Pauly ${ }^{30}$, J. Pearce ${ }^{170}$, M. Pedersen ${ }^{118}$, S. Pedraza Lopez ${ }^{168}$, R. Pedro ${ }^{125 a, 125 b}$, S.V. Peleganchuk ${ }^{108}$, D. Pelikan ${ }^{167}$, H. Peng ${ }^{33 b}$, B. Penning ${ }^{31}$, J. Penwell ${ }^{60}$, D.V. Perepelitsa ${ }^{25}$, E. Perez Codina ${ }^{160 a}$, M.T. Pérez García-Estañ ${ }^{168}$, V. Perez Reale ${ }^{35}$, L. Perini ${ }^{90 a}{ }^{90 b}$, H. Pernegger ${ }^{30}$, R. Perrino ${ }^{72 a}$, R. Peschke $^{42}$, V.D. Peshekhonov ${ }^{64}$, K. Peters ${ }^{30}$, R.F.Y. Peters ${ }^{83}$, B.A. Petersen ${ }^{30}$, T.C. Petersen ${ }^{36}$, E. Petit $^{42}$, A. Petridis ${ }^{147 a, 147 b}$, C. Petridou ${ }^{155}$, E. Petrolo ${ }^{133 a}$, F. Petrucci ${ }^{135 a, 135 b}$, N.E. Pettersson ${ }^{158}$, R. Pezoa ${ }^{32 b}$, P.W. Phillips ${ }^{130}$, G. Piacquadio ${ }^{144}$,

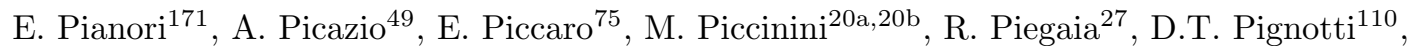
J.E. Pilcher ${ }^{31}$, A.D. Pilkington ${ }^{77}$, J. Pina ${ }^{125 a, 125 b, 125 d}$, M. Pinamonti ${ }^{165 a, 165 c, a c}$, A. Pinder ${ }^{119}$,

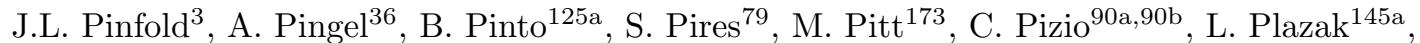
M.-A. Pleier ${ }^{25}$, V. Pleskot ${ }^{128}$, E. Plotnikova ${ }^{64}$, P. Plucinski ${ }^{147 a, 147 b}$, S. Poddar ${ }^{58 a}$, F. Podlyski ${ }^{34}$, R. Poettgen ${ }^{82}$, L. Poggioli ${ }^{116}$, D. Pohl ${ }^{21}$, M. Pohl ${ }^{49}$, G. Polesello ${ }^{120 a}$, A. Policicchio ${ }^{37 a, 37 b}$, R. Polifka ${ }^{159}$, A. Polini ${ }^{20 a}$, C.S. Pollard ${ }^{45}$, V. Polychronakos ${ }^{25}$, K. Pommès ${ }^{30}$, L. Pontecorvo ${ }^{133 a}$, B.G. Pope ${ }^{89}$, G.A. Popeneciu ${ }^{26 b}$, D.S. Popovic ${ }^{13 a}$, A. Poppleton ${ }^{30}$, X. Portell Bueso ${ }^{12}$, S. Pospisil ${ }^{127}$, K. Potamianos ${ }^{15}$, I.N. Potrap ${ }^{64}$, C.J. Potter ${ }^{150}$, C.T. Potter ${ }^{115}$, G. Poulard ${ }^{30}$, J. Poveda ${ }^{60}$, V. Pozdnyakov ${ }^{64}$, P. Pralavorio ${ }^{84}$, A. Pranko ${ }^{15}$, S. Prasad ${ }^{30}$, R. Pravahan ${ }^{8}$, S. Prell ${ }^{63}$, D. Price ${ }^{83}$, J. Price ${ }^{73}$, L.E. Price ${ }^{6}$, D. Prieur ${ }^{124}$, M. Primavera ${ }^{72 a}$, M. Proissl ${ }^{46}$, K. Prokofiev ${ }^{47}$, F. Prokoshin ${ }^{32 b}$, E. Protopapadaki ${ }^{137}$, S. Protopopescu ${ }^{25}$, J. Proudfoot ${ }^{6}$, M. Przybycien ${ }^{38 a}$, H. Przysiezniak ${ }^{5}$, E. Ptacek ${ }^{115}$, D. Puddu ${ }^{135 a, 135 b}$, E. Pueschel ${ }^{85}$, D. Puldon ${ }^{149}$, M. Purohit ${ }^{25, a d}$, P. Puzo ${ }^{116}$, J. Qian ${ }^{88}$, G. Qin ${ }^{53}$, Y. Qin ${ }^{83}$, A. Quadt ${ }^{54}$, D.R. Quarrie ${ }^{15}$, W.B. Quayle ${ }^{165 a, 165 b}$, M. Queitsch-Maitland ${ }^{83}$, D. Quilty ${ }^{53}$, A. Qureshi ${ }^{160 b}$, V. Radeka ${ }^{25}$, V. Radescu ${ }^{42}$, S.K. Radhakrishnan ${ }^{149}$, P. Radloff ${ }^{115}$, P. Rados $^{87}$, F. Ragusa ${ }^{90 a}$,90b, G. Rahal ${ }^{179}$, S. Rajagopalan ${ }^{25}$, M. Rammensee ${ }^{30}$, A.S. Randle-Conde ${ }^{40}$, C. Rangel-Smith ${ }^{167}$, K. Rao ${ }^{164}$, F. Rauscher ${ }^{99}$, T.C. Rave ${ }^{48}$, T. Ravenscroft ${ }^{53}$, M. Raymond ${ }^{30}$, A.L. Read $^{118}$, N.P. Readioff ${ }^{73}$, D.M. Rebuzzi ${ }^{120 a, 120 b}$, A. Redelbach ${ }^{175}$, G. Redlinger ${ }^{25}$, R. Reece ${ }^{138}$, K. Reeves ${ }^{41}$, L. Rehnisch ${ }^{16}$, H. Reisin ${ }^{27}$, M. Relich ${ }^{164}$, C. Rembser ${ }^{30}$, H. $\operatorname{Ren}^{33 a}$, Z.L. $\operatorname{Ren}^{152}$, A. Renaud ${ }^{116}$, M. Rescigno ${ }^{133 a}$, S. Resconi ${ }^{90 a}$, O.L. Rezanova ${ }^{108, t}$, P. Reznicek ${ }^{128}$, R. Rezvani ${ }^{94}$, R. Richter ${ }^{100}$, M. Ridel ${ }^{79}$, P. Rieck ${ }^{16}$, J. Rieger ${ }^{54}$, M. Rijssenbeek ${ }^{149}$, A. Rimoldi ${ }^{120 a, 120 b}$, L. Rinaldi ${ }^{20 a}$, E. Ritsch ${ }^{61}$, I. Riu $^{12}$, F. Rizatdinova ${ }^{113}$, E. Rizvi ${ }^{75}$, S.H. Robertson ${ }^{86, i}$, A. Robichaud-Veronneau ${ }^{86}$, D. Robinson ${ }^{28}$, J.E.M. Robinson ${ }^{83}$, A. Robson ${ }^{53}$, C. Roda ${ }^{123 a, 123 b}$, L. Rodrigues ${ }^{30}$, S. Roe ${ }^{30}$, O. Røhne ${ }^{118}$, S. Rolli ${ }^{162}$, A. Romaniouk ${ }^{97}$, M. Romano ${ }^{20 a, 20 b}$, E. Romero Adam ${ }^{168}$, N. Rompotis ${ }^{139}$,

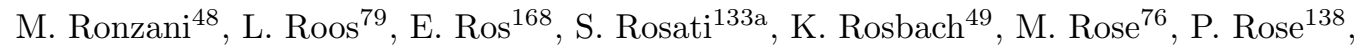
P.L. Rosendahl ${ }^{14}$, O. Rosenthal ${ }^{142}$, V. Rossetti ${ }^{147 a, 147 b}$, E. Rossi ${ }^{103 a, 103 b}$, L.P. Rossi ${ }^{50 a}$, R. Rosten ${ }^{139}$, M. Rotaru ${ }^{26 a}$, I. Roth ${ }^{173}$, J. Rothberg ${ }^{139}$, D. Rousseau ${ }^{116}$, C.R. Royon ${ }^{137}$, A. Rozanov ${ }^{84}$, Y. Rozen ${ }^{153}$, X. Ruan ${ }^{146 c}$, F. Rubbo ${ }^{12}$, I. Rubinskiy ${ }^{42}$, V.I. Rud ${ }^{98}$, C. Rudolph ${ }^{44}$, M.S. Rudolph ${ }^{159}$, F. Rühr ${ }^{48}$, A. Ruiz-Martinez ${ }^{30}$, Z. Rurikova ${ }^{48}$, N.A. Rusakovich ${ }^{64}$,

A. Ruschke ${ }^{99}$, J.P. Rutherfoord ${ }^{7}$, N. Ruthmann ${ }^{48}$, Y.F. Ryabov ${ }^{122}$, M. Rybar ${ }^{128}$, G. Rybkin ${ }^{116}$, N.C. Ryder ${ }^{119}$, A.F. Saavedra ${ }^{151}$, S. Sacerdoti ${ }^{27}$, A. Saddique ${ }^{3}$, I. Sadeh ${ }^{154}$,

H.F-W. Sadrozinski ${ }^{138}$, R. Sadykov ${ }^{64}$, F. Safai Tehrani1 ${ }^{133 a}$, H. Sakamoto ${ }^{156}$, Y. Sakurai ${ }^{172}$, G. Salamanna ${ }^{135 a, 135 b}$, A. Salamon ${ }^{134 a}$, M. Saleem ${ }^{112}$, D. Salek ${ }^{106}$, P.H. Sales De Bruin ${ }^{139}$, D. Salihagic ${ }^{100}$, A. Salnikov ${ }^{144}$, J. Salt ${ }^{168}$, D. Salvatore ${ }^{37 a, 37 b}$, F. Salvatore ${ }^{150}$, A. Salvucci ${ }^{105}$, A. Salzburger ${ }^{30}$, D. Sampsonidis ${ }^{155}$, A. Sanchez ${ }^{103 a, 103 b}$, J. Sánchez ${ }^{168}$, V. Sanchez Martinez ${ }^{168}$, H. Sandaker ${ }^{14}$, R.L. Sandbach ${ }^{75}$, H.G. Sander ${ }^{82}$, M.P. Sanders ${ }^{99}$, M. Sandhoff ${ }^{176}$, T. Sandoval ${ }^{28}$, C. Sandoval ${ }^{163}$, R. Sandstroem ${ }^{100}$, D.P.C. Sankey ${ }^{130}$, A. Sansoni ${ }^{47}$, C. Santoni ${ }^{34}$, R. Santonico ${ }^{134 a, 134 b}$, H. Santos ${ }^{125 a}$, I. Santoyo Castillo ${ }^{150}$, K. Sapp ${ }^{124}$, A. Sapronov ${ }^{64}$, J.G. Saraiva ${ }^{125 a, 125 d}$, B. Sarrazin ${ }^{21}$, G. Sartisohn ${ }^{176}$, O. Sasaki ${ }^{65}$, Y. Sasaki ${ }^{156}$, G. Sauvage ${ }^{5, *}$, 
E. $\operatorname{Sauvan}^{5}$, P. Savard ${ }^{159, d}$, D.O. Savu ${ }^{30}$, C. Sawyer ${ }^{119}$, L. Sawyer ${ }^{78, m}$, D.H. Saxon ${ }^{53}$, J. Saxon ${ }^{121}$, C. Sbarra ${ }^{20 a}$, A. Sbrizzi ${ }^{3}$, T. Scanlon ${ }^{77}$, D.A. Scannicchio ${ }^{164}$, M. Scarcella ${ }^{151}$, V. Scarfone ${ }^{37 a, 37 b}$, J. Schaarschmidt ${ }^{173}$, P. Schacht ${ }^{100}$, D. Schaefer ${ }^{30}$, R. Schaefer ${ }^{42}$, S. Schaepe ${ }^{21}$, S. Schaetzel ${ }^{58 b}$, U. Schäfer ${ }^{82}$, A.C. Schaffer ${ }^{116}$, D. Schaile ${ }^{99}$, R.D. Schamberger ${ }^{149}$, V. Scharf ${ }^{58 a}$, V.A. Schegelsky ${ }^{122}$, D. Scheirich ${ }^{128}$, M. Schernau ${ }^{164}$, M.I. Scherzer ${ }^{35}$, C. Schiavi ${ }^{50 a, 50 b,}$ J. Schieck ${ }^{99}$, C. Schillo ${ }^{48}$, M. Schioppa ${ }^{37 a, 37 b}$, S. Schlenker ${ }^{30}$, E. Schmidt ${ }^{48}$, K. Schmieden ${ }^{30}$, C. Schmitt ${ }^{82}$, S. Schmitt ${ }^{58 b}$, B. Schneider ${ }^{17}$, Y.J. Schnellbach ${ }^{73}$, U. Schnoor ${ }^{44}$, L. Schoeffel ${ }^{137}$, A. Schoening ${ }^{58 b}$, B.D. Schoenrock ${ }^{89}$, A.L.S. Schorlemmer ${ }^{54}$, M. Schott ${ }^{82}$, D. Schouten ${ }^{160 a}$, J. Schovancova ${ }^{25}$, S. Schramm ${ }^{159}$, M. Schreyer ${ }^{175}$, C. Schroeder ${ }^{82}$, N. Schuh ${ }^{82}$, M.J. Schultens ${ }^{21}$, H.-C. Schultz-Coulon ${ }^{58 a}$, H. Schulz ${ }^{16}$, M. Schumacher ${ }^{48}$, B.A. Schumm ${ }^{138}$, Ph. Schune ${ }^{137}$, C. Schwanenberger ${ }^{83}$, A. Schwartzman ${ }^{144}$, Ph. Schwegler ${ }^{100}$, Ph. Schwemling ${ }^{137}$,

R. Schwienhorst ${ }^{89}$, J. Schwindling ${ }^{137}$, T. Schwindt ${ }^{21}$, M. Schwoerer ${ }^{5}$, F.G. Sciacca ${ }^{17}$, E. Scifo ${ }^{116}$, G. Sciolla ${ }^{23}$, W.G. Scott ${ }^{130}$, F. Scuri ${ }^{123 a, 123 b}$, F. Scutti ${ }^{21}$, J. Searcy ${ }^{88}$, G. Sedov ${ }^{42}$, E. Sedykh ${ }^{122}$, S.C. Seidel ${ }^{104}$, A. Seiden ${ }^{138}$, F. Seifert ${ }^{127}$, J.M. Seixas ${ }^{24 a}$, G. Sekhniaidze ${ }^{103 a}$, S.J. Sekula ${ }^{40}$, K.E. Selbach ${ }^{46}$, D.M. Seliverstov ${ }^{122, *}$, G. Sellers ${ }^{73}$, N. Semprini-Cesari ${ }^{20 a, 20 b}$, C. Serfon ${ }^{30}$, L. Serin ${ }^{116}$, L. Serkin ${ }^{54}$, T. Serre ${ }^{84}$, R. Seuster ${ }^{160 a}$, H. Severini ${ }^{112}$, T. Sfiligoj ${ }^{74}$, F. Sforza ${ }^{100}$, A. Sfyrla ${ }^{30}$, E. Shabalina ${ }^{54}$, M. Shamim ${ }^{115}$, L.Y. Shan ${ }^{33 a}$, R. Shang ${ }^{166}$, J.T. Shank ${ }^{22}$, M. Shapiro ${ }^{15}$, P.B. Shatalov ${ }^{96}$, K. Shaw ${ }^{165 a, 165 b}$, C.Y. Shehu ${ }^{150}$, P. Sherwood ${ }^{77}$, L. Shi ${ }^{152, a e}$, S. Shimizu ${ }^{66}$, C.O. Shimmin ${ }^{164}$, M. Shimojima ${ }^{101}$, M. Shiyakova ${ }^{64}$, A. Shmeleva ${ }^{95}$, M.J. Shochet ${ }^{31}$, D. Short ${ }^{119}$, S. Shrestha ${ }^{63}$, E. Shulga ${ }^{97}$, M.A. Shupe ${ }^{7}$, S. Shushkevich ${ }^{42}$, P. Sicho ${ }^{126}$, O. Sidiropoulou ${ }^{155}$, D. Sidorov ${ }^{113}$, A. Sidoti133a ${ }^{132}$ F. Siegert ${ }^{44}$, Dj. Sijacki ${ }^{13 a}$, J. Silva ${ }^{125 a, 125 d}$, Y. Silver ${ }^{154}$, D. Silverstein ${ }^{144}$, S.B. Silverstein ${ }^{147 a}$, V. Simak ${ }^{127}$, O. Simard ${ }^{5}$, Lj. Simic ${ }^{13 a}$, S. Simion ${ }^{116}$, E. Simioni ${ }^{82}$, B. Simmons ${ }^{77}$, R. Simoniello ${ }^{90 a, 90 b}$, M. Simonyan ${ }^{36}$, P. Sinervo ${ }^{159}$, N.B. Sinev ${ }^{115}$, V. Sipica ${ }^{142}$, G. Siragusa ${ }^{175}$, A. Sircar ${ }^{78}$, A.N. Sisakyan ${ }^{64, *}$, S.Yu. Sivoklokov ${ }^{98}$, J. Sjölin ${ }^{147 a, 147 b}$, T.B. Sjursen ${ }^{14}$, H.P. Skottowe ${ }^{57}$, K.Yu. Skovpen ${ }^{108}$, P. Skubic ${ }^{112}$, M. Slater ${ }^{18}$, T. Slavicek ${ }^{127}$, K. Sliwa ${ }^{162}$, V. Smakhtin ${ }^{173}$, B.H. Smart ${ }^{46}$, L. Smestad ${ }^{14}$, S.Yu. Smirnov ${ }^{97}$, Y. Smirnov ${ }^{97}$, L.N. Smirnova ${ }^{98, a f}$, O. Smirnova ${ }^{80}$, K.M. Smith ${ }^{53}$, M. Smizanska ${ }^{71}$, K. Smolek ${ }^{127}$, A.A. Snesarev ${ }^{95}$, G. Snidero ${ }^{75}$, S. Snyder ${ }^{25}$, R. Sobie ${ }^{170, i}$, F. Socher ${ }^{44}$, A. Soffer ${ }^{154}$, D.A. Soh ${ }^{152, a e}$, C.A. Solans ${ }^{30}$, M. Solar ${ }^{127}$, J. Solc ${ }^{127}$, E.Yu. Soldatov ${ }^{97}$, U. Soldevila ${ }^{168}$, A.A. Solodkov ${ }^{129}$, A. Soloshenko ${ }^{64}$, O.V. Solovyanov ${ }^{129}$, V. Solovyev ${ }^{122}$, P. Sommer ${ }^{48}$, H.Y. Song ${ }^{33 b}$, N. Soni ${ }^{1}$, A. Sood $^{15}$, A. Sopczak ${ }^{127}$, B. Sopko ${ }^{127}$, V. Sopko ${ }^{127}$, V. Sorin ${ }^{12}$, M. Sosebee ${ }^{8}$, R. Soualah ${ }^{165 a, 165 c}$, P. Soueid ${ }^{94}$, A.M. Soukharev ${ }^{108}$, D. South ${ }^{42}$, S. Spagnolo ${ }^{72 a, 72 b}$, F. Spanò ${ }^{76}$, W.R. Spearman ${ }^{57}$, F. Spettel ${ }^{100}$, R. Spighi ${ }^{20 a}$, G. Spigo ${ }^{30}$, L.A. Spiller ${ }^{87}$, M. Spousta ${ }^{128}$, T. Spreitzer ${ }^{159}$, B. Spurlock ${ }^{8}$, R.D. St. Denis ${ }^{53, *}$, S. Staerz ${ }^{44}$, J. Stahlman ${ }^{121}$, R. Stamen ${ }^{58 a}$, S. Stamm ${ }^{16}$, E. Stanecka ${ }^{39}$, R.W. Stanek ${ }^{6}$, C. Stanescu ${ }^{135 a}$, M. Stanescu-Bellu ${ }^{42}$, M.M. Stanitzki ${ }^{42}$, S. Stapnes ${ }^{118}$, E.A. Starchenko ${ }^{129}$, J. Stark ${ }^{55}$, P. Staroba ${ }^{126}$, P. Starovoitov ${ }^{42}$, R. Staszewski ${ }^{39}$, P. Stavina ${ }^{145 a, *}$, P. Steinberg ${ }^{25}$, B. Stelzer ${ }^{143}$, H.J. Stelzer ${ }^{30}$, O. Stelzer-Chilton ${ }^{160 a}$, H. Stenzel ${ }^{52}$, S. Stern ${ }^{100}$, G.A. Stewart ${ }^{53}$, J.A. Stillings ${ }^{21}$, M.C. Stockton ${ }^{86}$, M. Stoebe ${ }^{86}$, G. Stoicea ${ }^{26 a}$, P. Stolte ${ }^{54}$, S. Stonjek ${ }^{100}$, A.R. Stradling ${ }^{8}$, A. Straessner ${ }^{44}$, M.E. Stramaglia ${ }^{17}$, J. Strandberg ${ }^{148}$, S. Strandberg147a,147b , A. Strandlie ${ }^{118}$, E. Strauss ${ }^{144}$, M. Strauss ${ }^{112}$, P. Strizenec ${ }^{145 b}$, R. Ströhmer ${ }^{175}$, D.M. Strom ${ }^{115}$, R. Stroynowski ${ }^{40}$, A. Struebig ${ }^{105}$, S.A. Stucci ${ }^{17}$, B. Stugu ${ }^{14}$, N.A. Styles ${ }^{42}$, D. $\mathrm{Su}^{144}$, J. $\mathrm{Su}^{124}$, R. Subramaniam ${ }^{78}$, A. Succurro ${ }^{12}$, Y. Sugaya ${ }^{117}$, C. Suhr ${ }^{107}$, M. Suk ${ }^{127}$, V.V. Sulin ${ }^{95}$, S. Sultansoy ${ }^{4 c}$, T. Sumida ${ }^{67}$, S. Sun ${ }^{57}$, X. Sun ${ }^{33 a}$, J.E. Sundermann ${ }^{48}$, K. Suruliz ${ }^{140}$, G. Susinno ${ }^{37 a, 37 b}$, M.R. Sutton ${ }^{150}$, Y. Suzuki ${ }^{65}$, M. Svatos ${ }^{126}$, S. Swedish ${ }^{169}$, M. Swiatlowski ${ }^{144}$, I. Sykora ${ }^{145 a}$, T. Sykora ${ }^{128}$, D. Ta ${ }^{89}$, C. Taccini ${ }^{135 a, 135 b}$, K. Tackmann ${ }^{42}$, J. Taenzer ${ }^{159}$, A. Taffard ${ }^{164}$, R. Tafirout ${ }^{160 a}$, N. Taiblum ${ }^{154}$, H. Takai ${ }^{25}$, R. Takashima ${ }^{68}$, H. Takeda ${ }^{66}$, T. Takeshita ${ }^{141}$, Y. Takubo ${ }^{65}$, M. Talby ${ }^{84}$, A.A. Talyshev ${ }^{108, t}$, J.Y.C. Tam ${ }^{175}$, K.G. $\operatorname{Tan}^{87}$, J. Tanaka ${ }^{156}$, R. Tanaka ${ }^{116}$, S. Tanaka ${ }^{132}$, 
S. Tanaka ${ }^{65}$, A.J. Tanasijczuk ${ }^{143}$, B.B. Tannenwald ${ }^{110}$, N. Tannoury ${ }^{21}$, S. Tapprogge ${ }^{82}$,

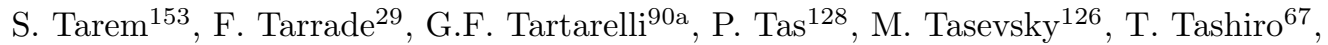
E. Tassi ${ }^{37 a, 37 b}$, A. Tavares Delgado ${ }^{125 a, 125 b}$, Y. Tayalati ${ }^{136 d}$, F.E. Taylor ${ }^{93}$, G.N. Taylor ${ }^{87}$, W. Taylor ${ }^{160 b}$, F.A. Teischinger ${ }^{30}$, M. Teixeira Dias Castanheira ${ }^{75}$, P. Teixeira-Dias ${ }^{76}$, K.K. Temming ${ }^{48}$, H. Ten Kate ${ }^{30}$, P.K. Teng ${ }^{152}$, J.J. Teoh ${ }^{117}$, S. Terada ${ }^{65}$, K. Terashi ${ }^{156}$, J. Terron ${ }^{81}$, S. Terzo ${ }^{100}$, M. Testa ${ }^{47}$, R.J. Teuscher ${ }^{159, i}$, J. Therhaag ${ }^{21}$, T. Theveneaux-Pelzer ${ }^{34}$, J.P. Thomas ${ }^{18}$, J. Thomas-Wilsker ${ }^{76}$, E.N. Thompson ${ }^{35}$, P.D. Thompson ${ }^{18}$, P.D. Thompson ${ }^{159}$, R.J. Thompson ${ }^{83}$, A.S. Thompson ${ }^{53}$, L.A. Thomsen $^{36}$, E. Thomson ${ }^{121}$, M. Thomson ${ }^{28}$, W.M. Thong ${ }^{87}$, R.P. Thun ${ }^{88, *}$, F. Tian $^{35}$, M.J. Tibbetts ${ }^{15}$, V.O. Tikhomirov ${ }^{95, a g}$, Yu.A. Tikhonov ${ }^{108, t}$, S. Timoshenko ${ }^{97}$, E. Tiouchichine ${ }^{84}$, P. Tipton ${ }^{177}$, S. Tisserant ${ }^{84}$, T. Todorov ${ }^{5}$, S. Todorova-Nova ${ }^{128}$, B. Toggerson ${ }^{7}$, J. Tojo ${ }^{69}$, S. Tokár ${ }^{145 a}$, K. Tokushuku ${ }^{65}$, K. Tollefson ${ }^{89}$, L. Tomlinson ${ }^{83}$, M. Tomoto ${ }^{102}$, L. Tompkins ${ }^{31}$, K. Toms ${ }^{104}$, N.D. Topilin ${ }^{64}$, E. Torrence ${ }^{115}$, H. Torres ${ }^{143}$, E. Torró Pastor ${ }^{168}$, J. Toth ${ }^{84, a h}$, F. Touchard ${ }^{84}$, D.R. Tovey ${ }^{140}$, H.L. $\operatorname{Tran}^{116}$, T. Trefzger ${ }^{175}$, L. Tremblet ${ }^{30}$, A. Tricoli ${ }^{30}$, I.M. Trigger ${ }^{160 a}$, S. Trincaz-Duvoid ${ }^{79}$, M.F. Tripiana ${ }^{12}$, W. Trischuk ${ }^{159}$, B. Trocmé ${ }^{55}$, C. Troncon ${ }^{90 a}$, M. Trottier-McDonald ${ }^{143}$, M. Trovatelli ${ }^{135 a, 135 b}$, P. True ${ }^{89}$, M. Trzebinski ${ }^{39}$, A. Trzupek $^{39}$, C. Tsarouchas ${ }^{30}$, J.C-L. Tseng ${ }^{119}$, P.V. Tsiareshka ${ }^{91}$, D. Tsionou ${ }^{137}$, G. Tsipolitis ${ }^{10}$, N. Tsirintanis ${ }^{9}$, S. Tsiskaridze ${ }^{12}$, V. Tsiskaridze ${ }^{48}$, E.G. Tskhadadze ${ }^{51 a}$, I.I. Tsukerman ${ }^{96}$, V. Tsulaia ${ }^{15}$, S. Tsuno ${ }^{65}$, D. Tsybychev ${ }^{149}$, A. Tudorache ${ }^{26 a}$, V. Tudorache ${ }^{26 a}$, A.N. Tuna ${ }^{121}$, S.A. Tupputi ${ }^{20 a, 20 b}$, S. Turchikhin ${ }^{98, a f}$, D. Turecek ${ }^{127}$, I. Turk Cakir ${ }^{4 d}$, R. Turra ${ }^{90 a, 90 b}$, P.M. Tuts ${ }^{35}$, A. Tykhonov ${ }^{49}$, M. Tylmad ${ }^{147 a, 147 b}$, M. Tyndel ${ }^{130}$, K. Uchida ${ }^{21}$, I. Ueda $^{156}$,

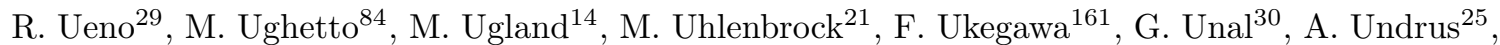
G. Unel ${ }^{164}$, F.C. Ungaro ${ }^{48}$, Y. Unno ${ }^{65}$, C. Unverdorben ${ }^{99}$, D. Urbaniec ${ }^{35}$, P. Urquijo ${ }^{87}$, G. Usai ${ }^{8}$, A. Usanova ${ }^{61}$, L. Vacavant ${ }^{84}$, V. Vacek ${ }^{127}$, B. Vachon ${ }^{86}$, N. Valencic ${ }^{106}$, S. Valentinettiina,20b, A. Valero ${ }^{168}$, L. Valery ${ }^{34}$, S. Valkar ${ }^{128}$, E. Valladolid Gallego ${ }^{168}$, S. Vallecorsa ${ }^{49}$, J.A. Valls Ferrer ${ }^{168}$, W. Van Den Wollenberg ${ }^{106}$, P.C. Van Der Deijl ${ }^{106}$, R. van der Geer ${ }^{106}$, H. van der Graaf ${ }^{106}$, R. Van Der Leeuw ${ }^{106}$, D. van der $\mathrm{Ster}^{30}$, N. van $\operatorname{Eldik}^{30}$, P. van Gemmeren ${ }^{6}$, J. Van Nieuwkoop ${ }^{143}$, I. van Vulpen ${ }^{106}$, M.C. van $W{ }^{2}{ }^{30}$, M. Vanadia ${ }^{133 a, 133 b}$, W. Vandelli ${ }^{30}$, R. Vanguri ${ }^{121}$, A. Vaniachine ${ }^{6}$, P. Vankov $^{42}$, F. Vannucci ${ }^{79}$, G. $\operatorname{Vardanyan}^{178}$, R. Vari ${ }^{133 a}$,

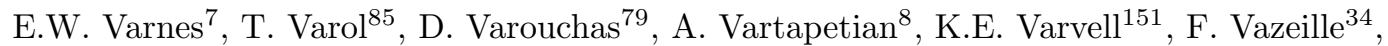

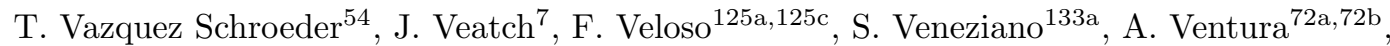
D. Ventura ${ }^{85}$, M. Venturi ${ }^{170}$, N. Venturi ${ }^{159}$, A. Venturini ${ }^{23}$, V. Vercesi ${ }^{120 a}$, M. Verducci ${ }^{133 a, 133 b}$, W. Verkerke ${ }^{106}$, J.C. Vermeulen ${ }^{106}$, A. Vest ${ }^{44}$, M.C. Vetterli ${ }^{143, d}$, O. Viazlo ${ }^{80}$, I. Vichou ${ }^{166}$, T. Vickey ${ }^{146 c, a i}$, O.E. Vickey Boeriu ${ }^{146 c}$, G.H.A. Viehhauser ${ }^{119}$, S. Viel ${ }^{169}$, R. Vigne ${ }^{30}$, M. Villa ${ }^{20 a}, 20 b$, M. Villaplana Perez ${ }^{90 a, 90 b}$, E. Vilucchi ${ }^{47}$, M.G. Vincter ${ }^{29}$, V.B. Vinogradov ${ }^{64}$, J. Virzi ${ }^{15}$, I. Vivarelli ${ }^{150}$, F. Vives Vaque ${ }^{3}$, S. Vlachos ${ }^{10}$, D. Vladoiu ${ }^{99}$, M. Vlasak ${ }^{127}$, A. Vogel $^{21}$, M. Vogel ${ }^{32 a}$, P. Vokac ${ }^{127}$, G. Volpi ${ }^{123 a, 123 b}$, M. Volpi ${ }^{87}$, H. von der Schmitt ${ }^{100}$,

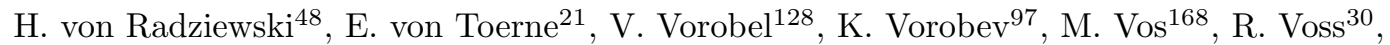
J.H. Vossebeld ${ }^{73}$, N. Vranjes ${ }^{137}$, M. Vranjes Milosavljevic ${ }^{13 a}$, V. Vrba ${ }^{126}$, M. Vreeswijk ${ }^{106}$, T. Vu Anh ${ }^{48}$, R. Vuillermet ${ }^{30}$, I. Vukotic ${ }^{31}$, Z. Vykydal ${ }^{127}$, P. Wagner ${ }^{21}$, W. Wagner ${ }^{176}$, H. Wahlberg ${ }^{70}$, S. Wahrmund ${ }^{44}$, J. Wakabayashi ${ }^{102}$, J. Walder ${ }^{71}$, R. Walker ${ }^{99}$, W. Walkowiak ${ }^{142}$, R. Wall ${ }^{177}$, P. Waller ${ }^{73}$, B. Walsh ${ }^{177}$, C. Wang ${ }^{152, a j}$, C. Wang ${ }^{45}$, F. Wang ${ }^{174}$, H. Wang ${ }^{15}$, H. Wang ${ }^{40}$, J. Wang ${ }^{42}$, J. Wang ${ }^{33 a}$, K. Wang ${ }^{86}$, R. Wang ${ }^{104}$, S.M. Wang ${ }^{152}$, T. Wang ${ }^{21}$, X. Wang ${ }^{177}$, C. Wanotayaroj ${ }^{115}$, A. Warburton ${ }^{86}$, C.P. Ward ${ }^{28}$, D.R. Wardrope ${ }^{77}$, M. Warsinsky ${ }^{48}$, A. Washbrook ${ }^{46}$, C. Wasicki ${ }^{42}$, P.M. Watkins ${ }^{18}$, A.T. Watson ${ }^{18}$, I.J. Watson ${ }^{151}$, M.F. Watson ${ }^{18}$, G. Watts ${ }^{139}$, S. Watts ${ }^{83}$, B.M. Waugh ${ }^{77}$, S. Webb ${ }^{83}$, M.S. Weber ${ }^{17}$,

S.W. Weber ${ }^{175}$, J.S. Webster ${ }^{31}$, A.R. Weidberg ${ }^{119}$, P. Weigell ${ }^{100}$, B. Weinert ${ }^{60}$, J. Weingarten ${ }^{54}$, C. Weiser ${ }^{48}$, H. Weits ${ }^{106}$, P.S. Wells ${ }^{30}$, T. Wenaus ${ }^{25}$, D. Wendland ${ }^{16}$, Z. Weng ${ }^{152, a e}$, T. Wengler ${ }^{30}$, 
S. Wenig ${ }^{30}$, N. Wermes ${ }^{21}$, M. Werner ${ }^{48}$, P. Werner ${ }^{30}$, M. Wessels ${ }^{58 a}$, J. Wetter ${ }^{162}$, K. Whalen ${ }^{29}$, A. White ${ }^{8}$, M.J. White ${ }^{1}$, R. White ${ }^{32 b}$, S. White ${ }^{123 a, 123 b}$, D. Whiteson ${ }^{164}$, D. Wicke ${ }^{176}$, F.J. Wickens ${ }^{130}$, W. Wiedenmann ${ }^{174}$, M. Wielers ${ }^{130}$, P. Wienemann ${ }^{21}$, C. Wiglesworth ${ }^{36}$, L.A.M. Wiik-Fuchs ${ }^{21}$, P.A. Wijeratne ${ }^{77}$, A. Wildauer ${ }^{100}$, M.A. Wildt ${ }^{42, a k}$, H.G. Wilkens ${ }^{30}$, J.Z. Will ${ }^{99}$, H.H. Williams ${ }^{121}$, S. Williams ${ }^{28}$, C. Willis ${ }^{89}$, S. Willocq ${ }^{85}$, A. Wilson ${ }^{88}$, J.A. Wilson ${ }^{18}$, I. Wingerter-Seez ${ }^{5}$, F. Winklmeier ${ }^{115}$, B.T. Winter ${ }^{21}$, M. Wittgen ${ }^{144}$, T. Wittig ${ }^{43}$, J. Wittkowski ${ }^{99}$, S.J. Wollstadt ${ }^{82}$, M.W. Wolter ${ }^{39}$, H. Wolters ${ }^{125 a, 125 c}$, B.K. Wosiek ${ }^{39}$,

J. Wotschack ${ }^{30}$, M.J. Woudstra ${ }^{83}$, K.W. Wozniak ${ }^{39}$, M. Wright ${ }^{53}$, M. Wu ${ }^{55}$, S.L. Wu ${ }^{174}$, X. Wu ${ }^{49}$, Y. Wu ${ }^{88}$, E. Wulf ${ }^{35}$, T.R. Wyatt ${ }^{83}$, B.M. Wynne ${ }^{46}$, S. Xella ${ }^{36}$, M. Xiao ${ }^{137}$, D. Xu ${ }^{33 a}$, L. Xu ${ }^{33 b, a l}$, B. Yabsley ${ }^{151}$, S. Yacoob ${ }^{146 b, a m}$, R. Yakabe ${ }^{66}$, M. Yamada ${ }^{65}$, H. Yamaguchi ${ }^{156}$, Y. Yamaguchi ${ }^{117}$, A. Yamamoto ${ }^{65}$, K. Yamamoto ${ }^{63}$, S. Yamamoto ${ }^{156}$, T. Yamamura ${ }^{156}$, T. Yamanaka ${ }^{156}$, K. Yamauchi ${ }^{102}$, Y. Yamazaki ${ }^{66}$, Z. Yan ${ }^{22}$, H. Yang ${ }^{33 e}$, H. Yang ${ }^{174}$, U.K. Yang ${ }^{83}$, Y. Yang ${ }^{110}$,

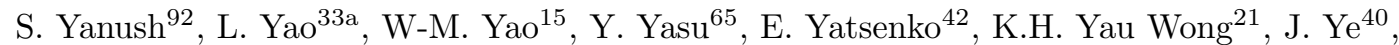
S. Ye ${ }^{25}$, I. Yeletskikh ${ }^{64}$, A.L. Yen ${ }^{57}$, E. Yildirim ${ }^{42}$, M. Yilmaz ${ }^{4 b}$, R. Yoosoofmiya ${ }^{124}$, K. Yorita ${ }^{172}$, R. Yoshida ${ }^{6}$, K. Yoshihara ${ }^{156}$, C. Young ${ }^{144}$, C.J.S. Young ${ }^{30}$, S. Youssef ${ }^{22}$, D.R. Yu ${ }^{15}$, J. Yu $^{8}$, J.M. $\mathrm{Yu}^{88}$, J. Yu ${ }^{113}$, L. Yuan ${ }^{66}$, A. Yurkewicz ${ }^{107}$, I. Yusuff ${ }^{28, a n}$, B. Zabinski ${ }^{39}$, R. Zaidan ${ }^{62}$, A.M. Zaitsev ${ }^{129, a a}$, A. Zaman ${ }^{149}$, S. Zambito ${ }^{23}$, L. Zanello ${ }^{133 a, 133 b}$, D. Zanzi ${ }^{100}$, C. Zeitnitz ${ }^{176}$, M. Zeman ${ }^{127}$, A. Zemla ${ }^{38 a}$, K. Zengel ${ }^{23}$, O. Zenin ${ }^{129}$, T. Ženišs ${ }^{145 a}$, D. Zerwas ${ }^{116}$, G. Zevi della Porta ${ }^{57}$, D. Zhang ${ }^{88}$, F. Zhang ${ }^{174}$, H. Zhang ${ }^{89}$, J. Zhang ${ }^{6}$, L. Zhang ${ }^{152}$, X. Zhang ${ }^{33 d}$, Z. Zhang ${ }^{116}$, Z. Zhao ${ }^{33 b}$, A. Zhemchugov ${ }^{64}$, J. Zhong ${ }^{119}$, B. Zhou ${ }^{88}$, L. Zhou ${ }^{35}$, N. Zhou ${ }^{164}$, C.G. Zhu ${ }^{33 d}$, H. Zhu ${ }^{33 a}$, J. Zhu ${ }^{88}$, Y. Zhu ${ }^{33 b}$, X. Zhuang ${ }^{33 a}$, K. Zhukov ${ }^{95}$, A. Zibell ${ }^{175}$, D. Zieminska ${ }^{60}$, N.I. Zimine ${ }^{64}$, C. Zimmermann ${ }^{82}$, R. Zimmermann ${ }^{21}$, S. Zimmermann ${ }^{21}$, S. Zimmermann ${ }^{48}$, Z. Zinonos ${ }^{54}$, M. Ziolkowski ${ }^{142}$, G. Zobernig ${ }^{174}$, A. Zoccoli ${ }^{20 a, 20 b}$, M. zur Nedden ${ }^{16}$, G. Zurzolo ${ }^{103 a, 103 b}$, V. Zutshi ${ }^{107}$ and L. Zwalinski ${ }^{30}$.

1 Department of Physics, University of Adelaide, Adelaide, Australia

2 Physics Department, SUNY Albany, Albany NY, United States of America

3 Department of Physics, University of Alberta, Edmonton AB, Canada

4 (a) Department of Physics, Ankara University, Ankara; ${ }^{(b)}$ Department of Physics, Gazi University, Ankara; ${ }^{(c)}$ Division of Physics, TOBB University of Economics and Technology, Ankara; ${ }^{(d)}$ Turkish Atomic Energy Authority, Ankara, Turkey

5 LAPP, CNRS/IN2P3 and Université de Savoie, Annecy-le-Vieux, France

6 High Energy Physics Division, Argonne National Laboratory, Argonne IL, United States of America

7 Department of Physics, University of Arizona, Tucson AZ, United States of America

8 Department of Physics, The University of Texas at Arlington, Arlington TX, United States of America

9 Physics Department, University of Athens, Athens, Greece

10 Physics Department, National Technical University of Athens, Zografou, Greece

11 Institute of Physics, Azerbaijan Academy of Sciences, Baku, Azerbaijan

12 Institut de Física d'Altes Energies and Departament de Física de la Universitat Autònoma de Barcelona, Barcelona, Spain

13 (a) Institute of Physics, University of Belgrade, Belgrade; ${ }^{(b)}$ Vinca Institute of Nuclear Sciences, University of Belgrade, Belgrade, Serbia

14 Department for Physics and Technology, University of Bergen, Bergen, Norway

15 Physics Division, Lawrence Berkeley National Laboratory and University of California, Berkeley CA, United States of America

16 Department of Physics, Humboldt University, Berlin, Germany

17 Albert Einstein Center for Fundamental Physics and Laboratory for High Energy Physics, University of Bern, Bern, Switzerland

18 School of Physics and Astronomy, University of Birmingham, Birmingham, United Kingdom 
19 (a) Department of Physics, Bogazici University, Istanbul; ${ }^{(b)}$ Department of Physics, Dogus University, Istanbul; ${ }^{(c)}$ Department of Physics Engineering, Gaziantep University, Gaziantep, Turkey

20 (a) INFN Sezione di Bologna; ${ }^{(b)}$ Dipartimento di Fisica e Astronomia, Università di Bologna, Bologna, Italy

21 Physikalisches Institut, University of Bonn, Bonn, Germany

22 Department of Physics, Boston University, Boston MA, United States of America

23 Department of Physics, Brandeis University, Waltham MA, United States of America

24 (a) Universidade Federal do Rio De Janeiro COPPE/EE/IF, Rio de Janeiro; ${ }^{(b)}$ Federal University of Juiz de Fora (UFJF), Juiz de Fora; ${ }^{(c)}$ Federal University of Sao Joao del Rei (UFSJ), Sao Joao del Rei; ${ }^{(d)}$ Instituto de Fisica, Universidade de Sao Paulo, Sao Paulo, Brazil

25 Physics Department, Brookhaven National Laboratory, Upton NY, United States of America

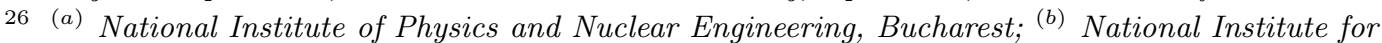
Research and Development of Isotopic and Molecular Technologies, Physics Department, Cluj Napoca; ${ }^{(c)}$ University Politehnica Bucharest, Bucharest; ${ }^{(d)}$ West University in Timisoara, Timisoara, Romania

27 Departamento de Física, Universidad de Buenos Aires, Buenos Aires, Argentina

28 Cavendish Laboratory, University of Cambridge, Cambridge, United Kingdom

29 Department of Physics, Carleton University, Ottawa ON, Canada

30 CERN, Geneva, Switzerland

31 Enrico Fermi Institute, University of Chicago, Chicago IL, United States of America

32 (a) Departamento de Fúsica, Pontificia Universidad Católica de Chile, Santiago; ${ }^{(b)}$ Departamento de Física, Universidad Técnica Federico Santa María, Valparaíso, Chile

33 (a) Institute of High Energy Physics, Chinese Academy of Sciences, Beijing; ${ }^{(b)}$ Department of Modern Physics, University of Science and Technology of China, Anhui; ${ }^{(c)}$ Department of Physics, Nanjing University, Jiangsu; ${ }^{(d)}$ School of Physics, Shandong University, Shandong; ${ }^{(e)}$ Physics Department, Shanghai Jiao Tong University, Shanghai, China

34 Laboratoire de Physique Corpusculaire, Clermont Université and Université Blaise Pascal and CNRS/IN2P3, Clermont-Ferrand, France

35 Nevis Laboratory, Columbia University, Irvington NY, United States of America

36 Niels Bohr Institute, University of Copenhagen, Kobenhavn, Denmark

37 (a) INFN Gruppo Collegato di Cosenza, Laboratori Nazionali di Frascati; ${ }^{(b)}$ Dipartimento di Fisica, Università della Calabria, Rende, Italy

38 (a) AGH University of Science and Technology, Faculty of Physics and Applied Computer Science, Krakow; ${ }^{(b)}$ Marian Smoluchowski Institute of Physics, Jagiellonian University, Krakow, Poland

39 The Henryk Niewodniczanski Institute of Nuclear Physics, Polish Academy of Sciences, Krakow, Poland

40 Physics Department, Southern Methodist University, Dallas TX, United States of America

41 Physics Department, University of Texas at Dallas, Richardson TX, United States of America

42 DESY, Hamburg and Zeuthen, Germany

43 Institut für Experimentelle Physik IV, Technische Universität Dortmund, Dortmund, Germany

44 Institut für Kern- und Teilchenphysik, Technische Universität Dresden, Dresden, Germany

45 Department of Physics, Duke University, Durham NC, United States of America

46 SUPA - School of Physics and Astronomy, University of Edinburgh, Edinburgh, United Kingdom

47 INFN Laboratori Nazionali di Frascati, Frascati, Italy

48 Fakultät für Mathematik und Physik, Albert-Ludwigs-Universität, Freiburg, Germany

49 Section de Physique, Université de Genève, Geneva, Switzerland

50 (a) INFN Sezione di Genova; ${ }^{(b)}$ Dipartimento di Fisica, Università di Genova, Genova, Italy

51 (a) E. Andronikashvili Institute of Physics, Iv. Javakhishvili Tbilisi State University, Tbilisi; ${ }^{(b)}$ High Energy Physics Institute, Tbilisi State University, Tbilisi, Georgia

52 II Physikalisches Institut, Justus-Liebig-Universität Giessen, Giessen, Germany

53 SUPA - School of Physics and Astronomy, University of Glasgow, Glasgow, United Kingdom 
II Physikalisches Institut, Georg-August-Universität, Göttingen, Germany

55 Laboratoire de Physique Subatomique et de Cosmologie, Université Grenoble-Alpes, CNRS/IN2P3, Grenoble, France

56 Department of Physics, Hampton University, Hampton VA, United States of America

57 Laboratory for Particle Physics and Cosmology, Harvard University, Cambridge MA, United States of America

58 (a) Kirchhoff-Institut für Physik, Ruprecht-Karls-Universität Heidelberg, Heidelberg; ${ }^{(b)}$ Physikalisches Institut, Ruprecht-Karls-Universität Heidelberg, Heidelberg; ${ }^{(c)}$ ZITI Institut für technische Informatik, Ruprecht-Karls-Universität Heidelberg, Mannheim, Germany

59 Faculty of Applied Information Science, Hiroshima Institute of Technology, Hiroshima, Japan

60 Department of Physics, Indiana University, Bloomington IN, United States of America

61 Institut für Astro- und Teilchenphysik, Leopold-Franzens-Universität, Innsbruck, Austria

${ }^{62}$ University of Iowa, Iowa City IA, United States of America

${ }^{63}$ Department of Physics and Astronomy, Iowa State University, Ames IA, United States of America

${ }^{64}$ Joint Institute for Nuclear Research, JINR Dubna, Dubna, Russia

${ }^{65}$ KEK, High Energy Accelerator Research Organization, Tsukuba, Japan

${ }^{66}$ Graduate School of Science, Kobe University, Kobe, Japan

67 Faculty of Science, Kyoto University, Kyoto, Japan

68 Kyoto University of Education, Kyoto, Japan

69 Department of Physics, Kyushu University, Fukuoka, Japan

70 Instituto de Física La Plata, Universidad Nacional de La Plata and CONICET, La Plata, Argentina

71 Physics Department, Lancaster University, Lancaster, United Kingdom

72 (a) INFN Sezione di Lecce; ${ }^{(b)}$ Dipartimento di Matematica e Fisica, Università del Salento, Lecce, Italy

73 Oliver Lodge Laboratory, University of Liverpool, Liverpool, United Kingdom

74 Department of Physics, Jožef Stefan Institute and University of Ljubljana, Ljubljana, Slovenia

75 School of Physics and Astronomy, Queen Mary University of London, London, United Kingdom

76 Department of Physics, Royal Holloway University of London, Surrey, United Kingdom

77 Department of Physics and Astronomy, University College London, London, United Kingdom

78 Louisiana Tech University, Ruston LA, United States of America

79 Laboratoire de Physique Nucléaire et de Hautes Energies, UPMC and Université Paris-Diderot and CNRS/IN2P3, Paris, France

80 Fysiska institutionen, Lunds universitet, Lund, Sweden

81 Departamento de Fisica Teorica C-15, Universidad Autonoma de Madrid, Madrid, Spain

82 Institut für Physik, Universität Mainz, Mainz, Germany

83 School of Physics and Astronomy, University of Manchester, Manchester, United Kingdom

${ }^{84}$ CPPM, Aix-Marseille Université and CNRS/IN2P3, Marseille, France

85 Department of Physics, University of Massachusetts, Amherst MA, United States of America

86 Department of Physics, McGill University, Montreal QC, Canada

87 School of Physics, University of Melbourne, Victoria, Australia

88 Department of Physics, The University of Michigan, Ann Arbor MI, United States of America

89 Department of Physics and Astronomy, Michigan State University, East Lansing MI, United States of America

90 (a) INFN Sezione di Milano; ${ }^{(b)}$ Dipartimento di Fisica, Università di Milano, Milano, Italy

91 B.I. Stepanov Institute of Physics, National Academy of Sciences of Belarus, Minsk, Republic of Belarus

92 National Scientific and Educational Centre for Particle and High Energy Physics, Minsk, Republic of Belarus

93 Department of Physics, Massachusetts Institute of Technology, Cambridge MA, United States of America

94 Group of Particle Physics, University of Montreal, Montreal QC, Canada

95 P.N. Lebedev Institute of Physics, Academy of Sciences, Moscow, Russia 
Institute for Theoretical and Experimental Physics (ITEP), Moscow, Russia

97 Moscow Engineering and Physics Institute (MEPhI), Moscow, Russia

98 D.V.Skobeltsyn Institute of Nuclear Physics, M.V.Lomonosov Moscow State University, Moscow, Russia

99 Fakultät für Physik, Ludwig-Maximilians-Universität München, München, Germany

100 Max-Planck-Institut für Physik (Werner-Heisenberg-Institut), München, Germany

101 Nagasaki Institute of Applied Science, Nagasaki, Japan

102 Graduate School of Science and Kobayashi-Maskawa Institute, Nagoya University, Nagoya, Japan

103 (a) INFN Sezione di Napoli; ${ }^{(b)}$ Dipartimento di Fisica, Università di Napoli, Napoli, Italy

104 Department of Physics and Astronomy, University of New Mexico, Albuquerque NM, United States of America

105 Institute for Mathematics, Astrophysics and Particle Physics, Radboud University Nijmegen/Nikhef, Nijmegen, Netherlands

106 Nikhef National Institute for Subatomic Physics and University of Amsterdam, Amsterdam, Netherlands

107 Department of Physics, Northern Illinois University, DeKalb IL, United States of America

108 Budker Institute of Nuclear Physics, SB RAS, Novosibirsk, Russia

109 Department of Physics, New York University, New York NY, United States of America

110 Ohio State University, Columbus OH, United States of America

111 Faculty of Science, Okayama University, Okayama, Japan

112 Homer L. Dodge Department of Physics and Astronomy, University of Oklahoma, Norman OK, United States of America

113 Department of Physics, Oklahoma State University, Stillwater OK, United States of America

114 Palacký University, RCPTM, Olomouc, Czech Republic

115 Center for High Energy Physics, University of Oregon, Eugene OR, United States of America

116 LAL, Université Paris-Sud and CNRS/IN2P3, Orsay, France

117 Graduate School of Science, Osaka University, Osaka, Japan

118 Department of Physics, University of Oslo, Oslo, Norway

119 Department of Physics, Oxford University, Oxford, United Kingdom

120 (a) INFN Sezione di Pavia; ${ }^{(b)}$ Dipartimento di Fisica, Università di Pavia, Pavia, Italy

121 Department of Physics, University of Pennsylvania, Philadelphia PA, United States of America

122 Petersburg Nuclear Physics Institute, Gatchina, Russia

123 (a) INFN Sezione di Pisa; ${ }^{(b)}$ Dipartimento di Fisica E. Fermi, Università di Pisa, Pisa, Italy

124 Department of Physics and Astronomy, University of Pittsburgh, Pittsburgh PA, United States of America

125 (a) Laboratorio de Instrumentacao e Fisica Experimental de Particulas - LIP, Lisboa; ${ }^{(b)}$ Faculdade de Ciências, Universidade de Lisboa, Lisboa; ${ }^{(c)}$ Department of Physics, University of Coimbra, Coimbra; ${ }^{(d)}$ Centro de Física Nuclear da Universidade de Lisboa, Lisboa; ${ }^{(e)}$ Departamento de Fisica, Universidade do Minho, Braga; ${ }^{(f)}$ Departamento de Fisica Teorica y del Cosmos and CAFPE, Universidad de Granada, Granada (Spain); ${ }^{(g)}$ Dep Fisica and CEFITEC of Faculdade de Ciencias e Tecnologia, Universidade Nova de Lisboa, Caparica, Portugal

126 Institute of Physics, Academy of Sciences of the Czech Republic, Praha, Czech Republic

127 Czech Technical University in Prague, Praha, Czech Republic

128 Faculty of Mathematics and Physics, Charles University in Prague, Praha, Czech Republic

129 State Research Center Institute for High Energy Physics, Protvino, Russia

130 Particle Physics Department, Rutherford Appleton Laboratory, Didcot, United Kingdom

131 Physics Department, University of Regina, Regina SK, Canada

132 Ritsumeikan University, Kusatsu, Shiga, Japan

133 (a) INFN Sezione di Roma; ${ }^{(b)}$ Dipartimento di Fisica, Sapienza Università di Roma, Roma, Italy

134 (a) INFN Sezione di Roma Tor Vergata; ${ }^{(b)}$ Dipartimento di Fisica, Università di Roma Tor Vergata, Roma, Italy

135 (a) INFN Sezione di Roma Tre; ${ }^{(b)}$ Dipartimento di Matematica e Fisica, Università Roma Tre, 
Roma, Italy

136 (a) Faculté des Sciences Ain Chock, Réseau Universitaire de Physique des Hautes Energies Université Hassan II, Casablanca; ${ }^{(b)}$ Centre National de l'Energie des Sciences Techniques Nucleaires, Rabat; ${ }^{(c)}$ Faculté des Sciences Semlalia, Université Cadi Ayyad, LPHEA-Marrakech; (d) Faculté des Sciences, Université Mohamed Premier and LPTPM, Oujda; ${ }^{(e)}$ Faculté des sciences, Université Mohammed V-Agdal, Rabat, Morocco

137 DSM/IRFU (Institut de Recherches sur les Lois Fondamentales de l'Univers), CEA Saclay (Commissariat à l'Energie Atomique et aux Energies Alternatives), Gif-sur-Yvette, France

138 Santa Cruz Institute for Particle Physics, University of California Santa Cruz, Santa Cruz CA, United States of America

139 Department of Physics, University of Washington, Seattle WA, United States of America

140 Department of Physics and Astronomy, University of Sheffield, Sheffield, United Kingdom

141 Department of Physics, Shinshu University, Nagano, Japan

142 Fachbereich Physik, Universität Siegen, Siegen, Germany

143 Department of Physics, Simon Fraser University, Burnaby BC, Canada

144 SLAC National Accelerator Laboratory, Stanford CA, United States of America

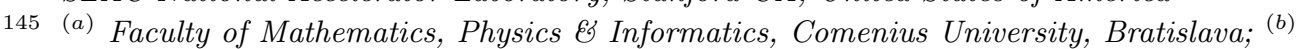

Department of Subnuclear Physics, Institute of Experimental Physics of the Slovak Academy of Sciences, Kosice, Slovak Republic

146 (a) Department of Physics, University of Cape Town, Cape Town; ${ }^{(b)}$ Department of Physics, University of Johannesburg, Johannesburg; ${ }^{(c)}$ School of Physics, University of the Witwatersrand, Johannesburg, South Africa

147 (a) Department of Physics, Stockholm University; ${ }^{(b)}$ The Oskar Klein Centre, Stockholm, Sweden

148 Physics Department, Royal Institute of Technology, Stockholm, Sweden

149 Departments of Physics $\&$ Astronomy and Chemistry, Stony Brook University, Stony Brook NY, United States of America

150 Department of Physics and Astronomy, University of Sussex, Brighton, United Kingdom

151 School of Physics, University of Sydney, Sydney, Australia

152 Institute of Physics, Academia Sinica, Taipei, Taiwan

153 Department of Physics, Technion: Israel Institute of Technology, Haifa, Israel

154 Raymond and Beverly Sackler School of Physics and Astronomy, Tel Aviv University, Tel Aviv, Israel

155 Department of Physics, Aristotle University of Thessaloniki, Thessaloniki, Greece

156 International Center for Elementary Particle Physics and Department of Physics, The University of Tokyo, Tokyo, Japan

157 Graduate School of Science and Technology, Tokyo Metropolitan University, Tokyo, Japan

158 Department of Physics, Tokyo Institute of Technology, Tokyo, Japan

159 Department of Physics, University of Toronto, Toronto ON, Canada

160 (a) TRIUMF, Vancouver BC; ${ }^{(b)}$ Department of Physics and Astronomy, York University, Toronto ON, Canada

161 Faculty of Pure and Applied Sciences, University of Tsukuba, Tsukuba, Japan

162 Department of Physics and Astronomy, Tufts University, Medford MA, United States of America

163 Centro de Investigaciones, Universidad Antonio Narino, Bogota, Colombia

164 Department of Physics and Astronomy, University of California Irvine, Irvine CA, United States of America

$165{ }^{(a)}$ INFN Gruppo Collegato di Udine, Sezione di Trieste, Udine; ${ }^{(b)}$ ICTP, Trieste; ${ }^{(c)}$ Dipartimento di Chimica, Fisica e Ambiente, Università di Udine, Udine, Italy

166 Department of Physics, University of Illinois, Urbana IL, United States of America

167 Department of Physics and Astronomy, University of Uppsala, Uppsala, Sweden

168 Instituto de Física Corpuscular (IFIC) and Departamento de Física Atómica, Molecular y Nuclear and Departamento de Ingeniería Electrónica and Instituto de Microelectrónica de Barcelona (IMB-CNM), University of Valencia and CSIC, Valencia, Spain 
170 Department of Physics and Astronomy, University of Victoria, Victoria BC, Canada

171 Department of Physics, University of Warwick, Coventry, United Kingdom

172 Waseda University, Tokyo, Japan

173 Department of Particle Physics, The Weizmann Institute of Science, Rehovot, Israel

174 Department of Physics, University of Wisconsin, Madison WI, United States of America

175 Fakultät für Physik und Astronomie, Julius-Maximilians-Universität, Würzburg, Germany

${ }_{176}$ Fachbereich C Physik, Bergische Universität Wuppertal, Wuppertal, Germany

177 Department of Physics, Yale University, New Haven CT, United States of America

178 Yerevan Physics Institute, Yerevan, Armenia

179 Centre de Calcul de l'Institut National de Physique Nucléaire et de Physique des Particules (IN2P3), Villeurbanne, France

a Also at Department of Physics, King's College London, London, United Kingdom

${ }^{b}$ Also at Institute of Physics, Azerbaijan Academy of Sciences, Baku, Azerbaijan

${ }^{c}$ Also at Particle Physics Department, Rutherford Appleton Laboratory, Didcot, United Kingdom

$d$ Also at TRIUMF, Vancouver BC, Canada

e Also at Department of Physics, California State University, Fresno CA, United States of America

$f$ Also at Tomsk State University, Tomsk, Russia

$g$ Also at CPPM, Aix-Marseille Université and CNRS/IN2P3, Marseille, France

${ }^{h}$ Also at Università di Napoli Parthenope, Napoli, Italy

${ }^{i}$ Also at Institute of Particle Physics (IPP), Canada

${ }^{j}$ Also at Department of Physics, St. Petersburg State Polytechnical University, St. Petersburg, Russia

${ }^{k}$ Also at Chinese University of Hong Kong, China

${ }^{l}$ Also at Department of Financial and Management Engineering, University of the Aegean, Chios, Greece

$m$ Also at Louisiana Tech University, Ruston LA, United States of America

$n$ Also at Institucio Catalana de Recerca i Estudis Avancats, ICREA, Barcelona, Spain

o Also at Department of Physics, The University of Texas at Austin, Austin TX, United States of America

${ }^{p}$ Also at Institute of Theoretical Physics, Ilia State University, Tbilisi, Georgia

${ }^{q}$ Also at CERN, Geneva, Switzerland

$r$ Also at Ochadai Academic Production, Ochanomizu University, Tokyo, Japan

$s$ Also at Manhattan College, New York NY, United States of America

${ }^{t}$ Also at Novosibirsk State University, Novosibirsk, Russia

${ }^{u}$ Also at Institute of Physics, Academia Sinica, Taipei, Taiwan

$v$ Also at LAL, Université Paris-Sud and CNRS/IN2P3, Orsay, France

w Also at Academia Sinica Grid Computing, Institute of Physics, Academia Sinica, Taipei, Taiwan

${ }^{x}$ Also at Laboratoire de Physique Nucléaire et de Hautes Energies, UPMC and Université Paris-Diderot and CNRS/IN2P3, Paris, France

y Also at School of Physical Sciences, National Institute of Science Education and Research, Bhubaneswar, India

$z$ Also at Dipartimento di Fisica, Sapienza Università di Roma, Roma, Italy

aa Also at Moscow Institute of Physics and Technology State University, Dolgoprudny, Russia

${ }^{a b}$ Also at section de Physique, Université de Genève, Geneva, Switzerland

ac Also at International School for Advanced Studies (SISSA), Trieste, Italy

ad Also at Department of Physics and Astronomy, University of South Carolina, Columbia SC, United States of America

ae Also at School of Physics and Engineering, Sun Yat-sen University, Guangzhou, China

af Also at Faculty of Physics, M.V.Lomonosov Moscow State University, Moscow, Russia

ag Also at Moscow Engineering and Physics Institute (MEPhI), Moscow, Russia

ah Also at Institute for Particle and Nuclear Physics, Wigner Research Centre for Physics, Budapest, 
Hungary

ai Also at Department of Physics, Oxford University, Oxford, United Kingdom

aj Also at Department of Physics, Nanjing University, Jiangsu, China

ak Also at Institut für Experimentalphysik, Universität Hamburg, Hamburg, Germany

al Also at Department of Physics, The University of Michigan, Ann Arbor MI, United States of America

am Also at Discipline of Physics, University of KwaZulu-Natal, Durban, South Africa

an Also at University of Malaya, Department of Physics, Kuala Lumpur, Malaysia

* Deceased 